\author{
Universidade de São Paulo \\ Instituto de Física
}

\title{
Equivalência quântica da formulação de primeira e segunda ordem da teoria de Yang-Mills e da gravitação
}

\author{
Sérgio Martins Filho
}

Orientador: Prof. Dr. Fernando Tadeu Caldeira Brandt

Dissertação de mestrado apresentada ao Instituto de Física da Universidade de São Paulo, como requisito parcial para a obtenção do título de Mestre em Ciências.

Banca Examinadora:

Prof. Dr. Fernando Tadeu Caldeira Brandt (IF-USP)

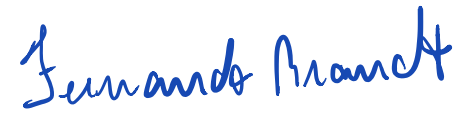

Prof. Dr. Diogo Rodrigues Boito (IFSC-USP)

Prof. Dr. Ilya Lvovich Shapiro (UFJF) 
FICHA CATALOGRÁFICA

Preparada pelo Serviço de Biblioteca e Informação do Instituto de Física da Universidade de São Paulo

Martins Filho, Sérgio

Equivalência quântica da formulação de primeira e segunda ordem da teoria de Yang-Mills e da gravitação. São Paulo, 2021.

Dissertação (Mestrado) - Universidade de São Paulo, Instituto de Física, Depto. de Física Experimental.

Orientador: Prof. Dr. Fernando Tadeu Caldeira Brandt

Área de Concentração: Física

Unitermos: 1. Teoria de gauge; 2. Yang-Mills; 3. Gravidade; 4. Formalismo de primeira ordem; 5. Equivalência quântica.

USP/IF/SBI-010/2021 


\title{
University of São Paulo
}

Physics Institute

\section{Quantum equivalence of the first - and second - order formulation of Yang-Mills theory and of gravity}

\author{
Sérgio Martins Filho
}

Supervisor: Prof. Dr. Fernando Tadeu Caldeira Brandt

Dissertation submitted to the Physics Institute of the University of São Paulo in partial fulfillment of the requirements for the degree of Master of Science.

Examining Committee:

Prof. Dr. Fernando Tadeu Caldeira Brandt (IF-USP)

Prof. Dr. Diogo Rodrigues Boito (IFSC-USP)

Prof. Dr. Ilya Lvovich Shapiro (UFJF)

São Paulo

2021 



\section{Agradecimentos}

Agradeço ao meu orientador Prof. Fernando T. C. Brandt que me acolheu desde o primeiro dia que pisei em São Paulo, agradeço-lhe pelo tempo que dedicou para me ensinar e pela sua atenção comigo nos bons momentos que podemos compartilhar até esse fatídico ano. Também preciso agradecer por todos os códigos-fonte que compartilhou comigo que só não me ajudaram muito no aprendizado de computação simbólica, mas também na realização desse trabalho.

Quero agradecer também ao Prof. D. G. C. McKeon e ao Prof. Josif Frenkel pelas colaborações que resultaram neste trabalho. Com a ajuda de vocês três cresci muito esse ano e foi uma grande honra estar junto com vocês fazendo o que eu mais gosto. Many thanks, Gerry!

Em especial, agradeço ao Prof. Frenkel que faz cada aula de eletrodinâmica parecer uma aula magna com sua sensibilidade e intuição física; e claro com alguma anedota interessadíssima... fazendo com que eu não faltasse (tantas) aulas.

Agradeço ao meu amigo, ex-colega do laboratório de informática na UFSC, Rômulo, cujas conversas, sobre os mais variados assuntos, me ajudaram imensamente nos piores e melhores momentos desses últimos anos. Além disso, o apoio e ajuda ao escrever essa dissertação. Agradeço também a minha colega Xirliane do IFUSP por breves, mas profundas, conversas que pudemos ter sobre nossos estudos.

Por todo suporte durante esse mestrado, agradeço ao pessoal da secretaria da pós do IFUSP, em especial à Andrea e ao Éber pela paciência.

Finalmente, agradeço a minha mãe, Solange, que me mostrou que eu era mais forte quando eu achei que não poderia mais continuar. Cujo suporte foi essencial, permitindo eu estar aqui escrevendo esses mesmos agradecimentos. Agradeço também aos meus irmãos, Suellen e Daniel, e ao meus sobrinhos, Beatriz e Davi, que sempre me ensinam a ser um ser humano melhor.

Quero agradecer também as minhas queridas colegas, Jaque e Cássia, que me incentivaram a continuar seguindo meus sonhos. À CAPES agradeço o auxílio financeiro necessário para realizar um desses sonhos.

O presente trabalho foi realizado com apoio da Coordenação de Aperfeiçoamento de Pessoal de Nível Superior - Brasil (CAPES) - Código de Financiamento 001. 



\section{RESUMO}

Estudamos a quantização da teoria de Yang-Mills nos formalismos de primeira e de segunda ordem e também na formulação diagonal do formalismo de primeira ordem. Consideramos também a quantização da gravitação, sem campos de matéria, em três formalismos análogos aos utilizados na teoria de Yang-Mills (utilizamos por simplicidade uma métrica de fundo de Minkowski). Demonstramos a equivalência quântica dessas formulações, tanto para teoria de Yang-Mills quanto para gravitação, utilizando o funcional gerador das funções de Green como definido via integrais de trajetória. Deduzimos um conjunto de identidades estruturais que relacionam as funções de Green no formalismo de primeira ordem (diagonal) com aquelas computadas no formalismo de segunda ordem. A equivalência quântica foi explicitamente verificada efetuando cálculos de funções de Green com campos de calibre externos, em todos os formalismos utilizados. Cálculos explícitos também foram realizados para verificar as identidades estruturais até ordem de um loop. Para a verificação dessas identidades levamos em conta divergências associadas a campos compostos. Usando uma extensão dos diagramas de Feynman mostramos que, na teoria de Yang-Mills, as identidades estruturais podem ser verificadas antes mesmo do cálculo explícito das integrais de um loop.

Palavras-chave: Equivalência quântica, Identidades estruturais, Formalismo de primeira ordem, Formulação diagonal, Teoria de Yang-Mills, Gravitação 



\section{ABstract}

We study the quantization of the Yang-Mills theory using both the first- and secondorder formalisms as well as the diagonal formulation of the first-order formalism. We also consider the quantization of gravitation, without matter fields, in three formalisms analogous to those used in the Yang-Mills theory (we use a Minkowski background metric for simplicity). Using the path integral formulation for the generating functional of Green's functions, we demonstrate the quantum equivalence of these formulations both for the YangMills theory and gravitation. We deduce a set of structural identities that relates Green's functions in the first-order (diagonal) formalism with the ones computed in second-order formalism. The quantum equivalence was explicitly verified by performing calculations of Green functions with external gauge fields, in all three formalisms. Additionally, explicit calculations were performed to verify the structural identities up to one-loop order. To verify these identities, we have taken into account divergences associated with composite fields. Using an extension of the Feynman diagrams we have shown that, in the Yang-Mills theory, the structural identities can be verified even before the calculation of the loop integrals is performed.

Keywords: Quantum equivalence, Structural identities, First-Order formalism, Diagonal formalism, Yang-Mills theory, Gravity 



\section{Lista de Tabelas}

6.1 Polo UV dos coeficientes da autoenergia mista $H \phi$ no calibre de DeDonder, definida na Eq. (6.58), em unidades de $i \kappa k^{2} I_{U V} \ldots \ldots \ldots$. . . . . . . . . 67

6.2 Polo UV dos coeficientes do propagador misto $H \phi$ no calibre de DeDonder, definido na Eq. (6.59), em unidades de $i \kappa^{3} I_{U V} \ldots \ldots \ldots$. . . . . . . . . . 67

6.3 Polo UV dos coeficientes da autoenergia $H H$ no calibre de DeDonder, definida na Eq. (6.62), em unidades de $k^{2} I_{U V} \ldots \ldots \ldots \ldots \ldots \ldots \ldots$

6.4 Polo UV dos coeficientes do propagador $H H$ no calibre de DeDonder, definido na Eq. (6.63), em unidades de $\kappa^{4} k^{2} I_{U V} \ldots \ldots \ldots \ldots \ldots \ldots$

A.1 Paralelos entre teorias de calibre e a gravitação . . . . . . . . . . . . . . . 77

B.1 Funcionais geradores e exemplos de diagramas gerados . . . . . . . . . . . . 86

E.1 Coeficientes da autoenergia mista $H \phi$, definida na Eq. (6.58), com unidades de $i \kappa k^{2} I_{U V} .113$

E.2 Polo UV dos coeficientes da autoenergia mista $H \phi$, definida na Eq. (6.58), com unidades de $i \kappa k^{2} I_{U V} \ldots \ldots \ldots \ldots$. . . . . . . . . . . . . . . . . . 114

E.3 Coeficientes da autoenergia mista $H H$, definida na Eq. (6.62), com unidades de $k^{2} I_{11} .115$

E.4 Polo UV dos coeficientes da autoenergia mista $H H$, definida na Eq. (6.62), com

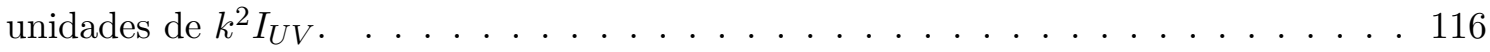

E.5 Coeficientes do propagador $H \phi$, definido na Eq. (6.59), com unidades de $\kappa^{3} I_{11} \ldots \ldots$

E.6 Polo UV dos coeficientes do propagador $H \phi$, definido na Eq. (6.59), com unidades de

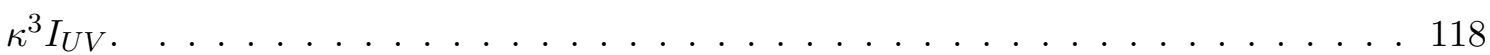

E.7 Coeficientes do propagador $H H$, definido na Eq. (6.63), com unidades de $\kappa^{4} k^{2} I_{11}$. 119

E.8 Polo UV dos coeficientes do propagador $H H$, definido na Eq. (6.63), com unidades

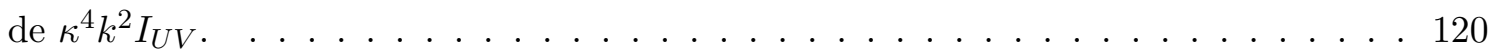





\section{Lista de Figuras}

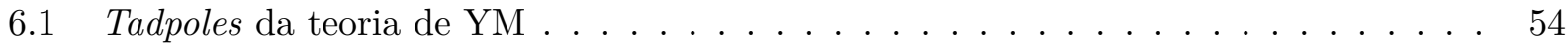

6.2 Diagrama pinched obtido a partir do propagador do campo $A_{\mu}^{a} \ldots \ldots \ldots \ldots 5$

6.3 Diagramas pinched no espaço de momento . . . . . . . . . . . . . . . . 56

6.4 Correção de um loop para o propagador misto $H A \ldots \ldots \ldots \ldots$

6.5 Correção de um loop para o propagador do campo $H_{\mu \nu}^{a} \ldots \ldots \ldots$. . . . . . . . 60

6.6 Diagramas pinched que contribuem para o lado direito das identidades (3.64b) e (3.64c) 62

6.7 Regra de Feynman para o vértice $(h A A) \ldots \ldots \ldots \ldots \ldots \ldots \ldots \ldots$

6.8 Diagramas de um loop com o campo composto $h_{\mu \nu}^{a}$ externo . . . . . . . . . . 63

6.9 Correção de um loop para o propagador misto $H \phi \ldots \ldots$. . . . . . . . . . . 66

6.10 Correção de um loop para o propagador do campo $H_{\mu \nu}^{\lambda} \ldots \ldots$. . . . . . . . 68

6.11 Diagrama pinched de um loop que contribui para o lado direito da Eq. (6.52). . . 70

6.12 Diagramas pinched que contribuem, no nível de um loop, para o lado direito da

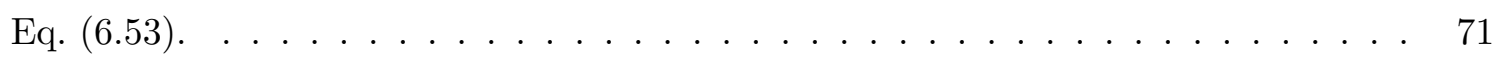

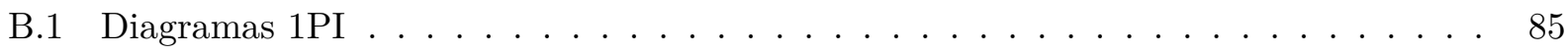

F.1 Diagramas de ordem árvore do espalhamento glúon-glúon no formalismo de segunda

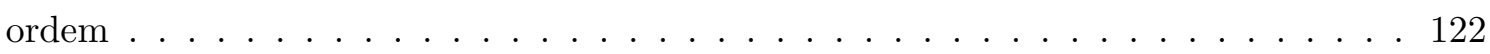

F.2 Canais s, t e u do espalhamento glúon-glúon no formalismo de segunda ordem. . . . 122

F.3 Diagrama (canal s) do tipo 0. O momento interno $q=p_{1}+p_{2}=-\left(p_{3}+p_{4}\right)$. . . 122

F.4 Diagramas (canal s) do tipo I. O momento interno $q=p_{1}+p_{2}=-\left(p_{3}+p_{4}\right)$. . . . 123

F.5 Diagramas (canal s) do tipo II. O momento interno $q=p_{1}+p_{2}=-\left(p_{3}+p_{4}\right) \ldots \ldots 123$ 



\section{Sumário}

\section{Lista de Tabelas}

\section{Lista de Figuras}

1 Introdução 1

2 Teoria de Yang-Mills $\quad 5$

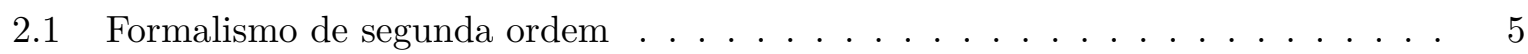

2.1.1 Quantização ............................. 6

2.1.2 Regras de Feynman . . . . . . . . . . . . . . . . . . . 8

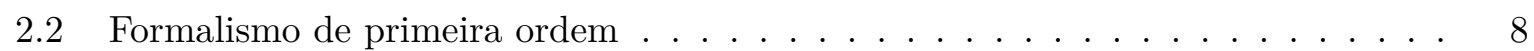

2.2 .1 Quantização . . . . . . . . . . . . . . . . . . . 9

2.2 .2 Regras de Feynman . . . . . . . . . . . . . . . . . . . . . . . . . . . . . . . . .

2.3 Formalismo diagonal . . . . . . . . . . . . . . . . . . . . . . 11

2.3 .1 Regras de Feynman . . . . . . . . . . . . . . . . . . 12

3 Equivalência Quântica: Teoria de Yang-Mills $\quad 15$

3.1 Equivalência parcial para o formalismo de primeira ordem . . . . . . . . . . . 15

3.2 Equivalência quântica para o formalismo de primeira ordem . . . . . . . . . . . . 17

3.2 .1 Condição de consistência . . . . . . . . . . . . . . . . . . 20

3.2 .2 Identidades estruturais . . . . . . . . . . . . . . . . . . 21

3.3 Equivalência parcial para o formalismo diagonal . . . . . . . . . . . . . . 25

3.4 Equivalência quântica para o formalismo diagonal . . . . . . . . . . . . . 25

3.4 .1 Identidades estruturais . . . . . . . . . . . . . . . . 27

3.5 Equivalência geral . . . . . . . . . . . . . . . . . . 28

4 Gravitação $\quad 31$

4.1 Ação de Einstein-Hilbert . . . . . . . . . . . . . . . . . . . . . 31

4.1 Quantização . . . . . . . . . . . . . . . . . . . 32

4.2 Formalismo de primeira ordem . . . . . . . . . . . . . . . . . 34

4.2 .1 Quantização . . . . . . . . . . . . . . . . . . 36

4.3 Formalismo diagonal . . . . . . . . . . . . . . . . . . . . 37 
$4.3 .1 \quad$ Regras de Feynman . . . . . . . . . . . . . . . . . . . . . . 39

5 Equivalência Quântica: Gravitação $\quad 41$

5.1 Equivalência parcial para o formalismo de primeira ordem . . . . . . . . . . . . 41

5.2 Equivalência quântica para o formalismo de Palatini . . . . . . . . . . . . . . . 43

5.3 Equivalência quântica para o formalismo diagonal . . . . . . . . . . . . . . 46

5.3 .1 Condição de consistência . . . . . . . . . . . . . . . . . . . 47

5.3 .2 Identidades estruturais . . . . . . . . . . . . . . . . . . . 49

5.4 Equivalência geral . . . . . . . . . . . . . . . . . . . . . 50

6 Verificação Explícita das Identidades Estruturais 53

6.1 Teoria de Yang-Mills . . . . . . . . . . . . . . . . . . . . . 53

6.1 .1 Verificação da identidade $(3.31) \ldots \ldots \ldots \ldots$. . . . . . . . 54

6.1 .2 Verificação das identidades $(3.35)$ e $(3.44) \ldots \ldots \ldots$. . . . . . . 56

6.1 .3 Correções quânticas em um loop . . . . . . . . . . . . . . . . . . 58

6.1 .4 Diagramas pinched . . . . . . . . . . . . . . . . . . 61

6.1.5 Resultados para o formalismo diagonal . . . . . . . . . . . . . . . . 64

6.2 Gravitação . . . . . . . . . . . . . . . . . . . . . . 65

6.2 .1 Ordem árvore . . . . . . . . . . . . . . . . . . . . 65

6.2 .2 Correções quânticas de um loop . . . . . . . . . . . . . . . . . 66

6.2.3 Correlações do campo composto $\Delta_{\mu \nu}^{\lambda}(x) \ldots \ldots \ldots$. . . . . . . . 69

$\begin{array}{lll}7 & \text { Considerações Finais } & 73\end{array}$

$\begin{array}{ll}\text { A Teorias de Calibre } & \mathbf{7 7}\end{array}$

A.1 Transformação de calibre . . . . . . . . . . . . . . . . . . . . . 78

A.2 Derivada covariante e o campo de calibre . . . . . . . . . . . . . . . 79

A.3 Teoria de Yang-Mills pura . . . . . . . . . . . . . . . . . . . . . 79

B Funcionais Geradores $\quad 81$

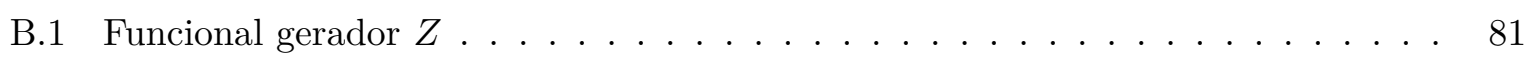

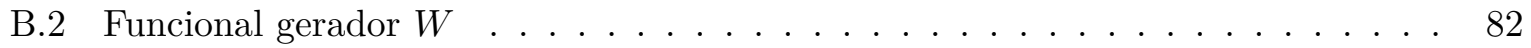

B.3 Ação efetiva . . . . . . . . . . . . . . . . . . . . . . . . 83

$\begin{array}{lll}\text { C } & \text { Regras de Feynman } & 87\end{array}$

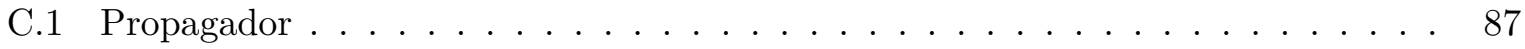

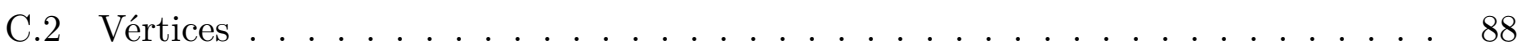

C.3 Teoria de Yang-Mills . . . . . . . . . . . . . . . . . . . . . . . . . . 89

C.3.1 Formalismo de segunda ordem . . . . . . . . . . . . . . . . . . . 89

C.3.2 Formalismo de primeira ordem . . . . . . . . . . . . . . . . . . . 91

C.3.3 Formalismo diagonal . . . . . . . . . . . . . . . . . . . . . 94

C.4 Gravitação . . . . . . . . . . . . . . . . . . . . . . . . . . . 96

C.4.1 Campos fantasmas $d_{\nu}$ e $\bar{d}_{\mu} \ldots \ldots \ldots \ldots \ldots$ 
C.4.2 Formalismo diagonal . . . . . . . . . . . . . . . . . . . . 96

C.4.3 Demais formalismos . . . . . . . . . . . . . . . . . 98

D Método para Computação de Diagramas de 1-Loop 101

D.1 Regularização dimensional . . . . . . . . . . . . . . . . . . . . . . . . 101

D.1.1 Uma integral básica . . . . . . . . . . . . . . . . . . . . . . . . . 102

D.1.2 Integral de potências . . . . . . . . . . . . . . . . . . . . 103

D.2 Redução de Passarino-Veltman . . . . . . . . . . . . . . . . . . . . . . . . 104

D.2.1 Procedimento geral . . . . . . . . . . . . . . . . . . . . . . 104

D.3 Bases tensoriais . . . . . . . . . . . . . . . . . . . . . . 105

D.3.1 Teoria de Yang-Mills . . . . . . . . . . . . . . . . . 106

D.3.2 Gravitação . . . . . . . . . . . . . . . . . . . . 106

D.4 Exemplo de código . . . . . . . . . . . . . . . . . . . . . 107

E Resultados Gerais para Gravitação $\quad 113$

F Amplitude $(A A A A)$ na Teoria de Yang-Mills $\quad 121$

F.1 Formalismo de segunda ordem . . . . . . . . . . . . . . . . . . . . . . . . 121

F.2 Formalismo de primeira ordem . . . . . . . . . . . . . . . . . . . . 121

F.3 Código do Mathematica $12 \ldots \ldots \ldots \ldots$. . . . . . . . . . . . 123

F.4 Discussão dos resultados . . . . . . . . . . . . . . . . . . . . . . . 124

Referências Bibliográficas $\quad 129$ 



\section{CAPÍTULO 1}

\section{INTRODUÇÃO}

Atualmente as teorias de calibre tomaram um espaço relevante no entendimento das interações fundamentais, por exemplo, o modelo padrão, uma das teorias mais bem sucedidas na descrição das interações fundamentais (exceto a gravitação) [1] e das partículas elementares que participam dos processos de interação. O modelo padrão é basicamente a junção de duas teorias de calibre: a teoria eletrofraca e a cromodinâmica quântica. Tais teorias são exemplos de uma classe especial de teoria de calibre, a teoria de Yang-Mills (YM).

A teoria de YM surgiu com o trabalho de Yang e Mills [2] na busca de uma descrição da interação forte. Embora a teoria original não tenha sido bem sucedida, a ideia básica considerada por Yang e Mills foi amplamente usada. O conceito básico da teoria de YM, sugerido originalmente pelos mesmos, é considerar uma teoria com invariância sobre transformações de calibre não abelianas, essas transformações são uma generalização das transformações de calibre usuais (abelianas) que aparecem na eletrodinâmica de Maxwell [3].

É amplamente conhecido que o modelo padrão não é compatível com a gravitação somente descrevendo três das quatro interações conhecidas. No entanto, notavelmente, a gravitação pode ser considerada como uma teoria de calibre assim como é o caso das teorias que descrevem as demais interações fundamentais. Para isso usamos a invariância da gravitação sobre transformações gerais de coordenadas [3] e elevamos essas transformações à transformações de calibre. Assim como a teoria de YM, a gravitação é uma teoria de calibre não abeliana. Embora haja diferenças devido a natureza da origem da invariância de calibre entre a teoria de YM e a gravitação também há diversas semelhanças que são consideradas neste trabalho.

Tanto a teoria de YM quanto a gravitação, nas suas formulações usuais, são teorias de "segunda ordem", isso é, as lagrangianas que descrevem tais teorias possuem termos com derivadas de segunda ordem. Tal dependência implica em regras de Feynman mais complicadas para o estudo perturbativo dessas teorias, contudo ambas teorias possuem uma formulação alternativa de "primeira ordem" [4]. O formalismo de primeira ordem de ambas teorias é mais vantajoso já que temos uma simplificação dos vértices dessas teorias. No caso da gravitação, temos uma simplificação muito mais revelante, pois passamos de infinitos vértices para apenas um vértice 
cúbico [5].

Para obter a forma de primeira ordem introduzimos um campo auxiliar (não físico) que interage de forma mais simples com o campo de calibre (físico), por outro lado também temos os não usuais propagadores mistos. Mais recentemente, mostrou-se que podemos remover os propagadores mistos do formalismo de primeira ordem [6] numa formulação híbrida entre o formalismo de primeira ordem e segunda ordem. Esse formalismo diagonal é mais revelante no caso da gravitação, uma vez que que a simplificação ainda é revelante na computação de correções quânticas de um ou mais loops.

O estudo perturbativo no formalismo de primeira ordem (e diagonal) já foi extensivamente considerado na literatura. Em [4], foram obtidos os resultados ao nível de um loop (ordem $\hbar$ ) para as autoenergias no formalismo de primeira ordem da teoria de YM; enquanto [7] mostra-se a eficácia do formalismo na computação de correções quânticas similares usando o formalismo de campo de fundo. A renormalização da teoria de YM na formulação de primeira ordem é considerada no artigo [8], já na formulação diagonal em [9].

Para gravitação, podemos mencionar [10-12] que usam o formalismo de primeira ordem para computação de correções quânticas. Mais recentemente, o artigo [5] que considerou a correção de um loop do tensor energia momento térmico no formalismo de primeira ordem mostrando a equivalência dos resultados com o formalismo de segunda ordem. No artigo [6], o resultado da autoenergia do gráviton é reproduzido usando o formalismo diagonal da gravitação.

Conforme $[4,6,13]$, o formalismo de primeira ordem (diagonal), da teoria de YM e da gravitação, é classicamente equivalente ao formalismo usual - o formalismo de segunda ordem considerando que não há presença de campos de matéria. Além disso, de acordo com $[5,6]$ tal equivalência também vale no nível quântico fazendo a quantização via integrais de trajetória com o funcional gerador das funções de Green $Z$, porém sem a presença de fontes. Sendo que as fontes, introduzidas inicialmente por Schwinger [14], possuem um papel muito importante para a geração de correlações a partir do funcional gerador, pois são as derivadas funcionais de $Z \mathrm{em}$ relação a essas fontes que "geram" as funções de Green.

Embora $[4-7,10,11]$ mostrem explicitamente que no nível de um loop as correções quânticas são iguais, isso é, uma verificação explícita da equivalência quântica, a equivalência geral - com fontes — ainda não tinha sido demonstrada formalmente.

Nos trabalhos publicados $[15,16]$ demostra-se a equivalência geral entre o formalismo de primeira ordem (diagonal) e o formalismo de segunda ordem da teoria de YM e da gravitação. Para isso mostramos a equivalência dos funcionais geradores $Z$ nos diferentes formalismos incluindo as fontes.

Além disso, deduzi-se um conjunto de identidades estruturais que relacionam as funções de Green do formalismo de primeira ordem (diagonal) com funções de Green do formalismo de segunda ordem. Entre essas identidades temos a igualdade entre as funções de Green do campo de calibre computadas nos três formalismos mostrando explicitamente a equivalência no nível quântico em qualquer ordem.

Nessas identidades aparecem correlações de campos compostos, isso é, um produto de mais de um campo no mesmo ponto do espaço-tempo, cujas divergências [17] são de origem diferente das 
usuais divergências ultravioletas [15] que surgem com os loops na expansão perturbativa. Tais divergências são essenciais para a verificação dessas identidades, como feito nos artigos [15, 16], em até um loop, mostrando que valem em qualquer calibre e qualquer dimensão.

Neste trabalho nosso principal objetivo é dar em detalhes os resultados publicados durante o mestrado nos artigos $[15,16]$ já mencionados acima. Além disso deduzimos que, para teoria de YM, podemos estender a verificação das identidades estruturais ao nível de integrando. Isso mostra que essas identidades são mais elementares que pensávamos deixando claro que valem para qualquer dimensão ou calibre.

No próximo capítulo iremos considerar a quantização da teoria de YM em três diferentes formalismos: o formalismo usual de segunda ordem, o formalismo de primeira ordem e formalismo diagonal. Também mostramos as regras de Feynman para cada um deles.

No terceiro capítulo demostramos a equivalência quântica com fontes dessas diferentes formulações da teoria de YM e deduzimos um conjunto de identidades estruturais relacionando as funções de Green do formalismo de primeira ordem (diagonal) com correlações do formalismo de segunda ordem, conforme feito em [15]. Para demostrar a equivalência geral usamos um ansatz inferido ao verificar a equivalência sem fontes sendo compatível com o artigo original [15]. Finalizamos com uma breve discussão sobre funcionais geradores generalizados induzidos pela equivalências dos formalismos considerados.

Seguindo para gravitação, no quarto capítulo, definimos o formalismo de segunda ordem dado pela ação de Einstein-Hilbert (EH) cuja quantização requer a introdução de um campo de fundo, por simplicidade, escolhemos trabalhar com um campo de fundo plano. O mesmo vale para a quantização da gravitação no formalismo de primeira ordem, também conhecido como formalismo de Palatini [18], e o formalismo diagonal, análogo ao da teoria de YM, no qual há uma simplificação semelhante ao formalismo de primeira ordem, porém sem a presença de propagadores mistos. No fim damos as regras de Feynman para o formalismo diagonal da gravitação.

No quinto capítulo demonstramos a equivalência quântica com fontes no caso da gravitação. Também obtemos um conjunto de identidades estruturais, embora semelhantes, são mais complicadas que as deduzidas no caso da teoria de YM, que relacionam as funções de Green do formalismo diagonal com o formalismo de segunda ordem da gravitação. Para essa demostração usamos um ansatz análogo ao usado no caso da teoria de YM que confere com os resultados publicados no artigo [16]. Da mesma forma que feito para teoria de YM discutimos sobre funcionais geradores generalizados no caso da gravitação.

Finalmente, no sexto capítulo verificamos explicitamente algumas das identidades estruturais deduzidas no terceiro e quinto capítulo até um loop. A verificação dessas identidades como nos artigos $[15,16]$ foram feitas usando computação simbólica. Os diagramas que são obtidos pela presença de campos compostos são do tipo "pinched". Um diagrama pinched é um diagrama que possui um ou mais pares de pernas externas no mesmo ponto do espaço-tempo, sendo semelhantes a loops, tanto graficamente quanto quantitativamente [15].

Para teoria de YM mostramos que os diagramas pinched podem ser computados como diagramas usuais ao estender as regras de Feynman adicionando um vértice auxiliar. Isso 
permitiu estender os resultados em [15, Appendix B] para o nível de integrando. Já no caso da gravitação reproduzimos os resultados do artigo [16] com algumas divergências devido a diferentes convenções.

Neste trabalho usaremos o sistema de unidades naturais em que $\hbar=c=1$, a assinatura da métrica de Minkowski é convencionada como $(1,-1,-1,-1)$. Além disso, usamos a notação de soma do Einstein, ou seja, índices repetidos denotam a soma sobre os mesmos. Usualmente índices gregos $\mu, \nu, \rho, \ldots$ vão de 0 até $d-1$, onde o índice " 0 " representa a dimensão temporal e $d$ é dimensão do espaço-tempo (em princípio $d=4$ ). Os índices latinos $a, b, c, \ldots$ geralmente são índices de cor do $S U(N)$ indo de 1 até $N^{2}-1$. 
Neste capítulo mostraremos a quantização da teoria de YM em três formulações:

- Formulação usual de segunda ordem.

- Formulação de primeira ordem.

- Formulação diagonal.

Para isso usaremos o formalismo de integrais de trajetória aplicando o procedimento de FaddeevPopov [19]. Além disso, damos as regras de Feymann nessas diferentes formulações que serão úteis posteriormente para os cálculos perturbativos realizados no capítulo 5 .

Usualmente a teoria de YM conta com a presença de campos de matéria, porém neste trabalho só iremos considerar a teoria pura, i.e. contendo somente o campo de calibre. Como os diferentes formalismos estudados somente modificam o setor do campo de calibre a introdução de campos de matéria é trivial.

\subsection{Formalismo de segunda ordem}

Nessa seção mostraremos concisamente a quantização da teoria de YM na sua formulação usual, como é visto em diversos livros [20-22]. Um estudo mais elementar e completo da quantização via integral de trajetória é dado em diversos livros como [17, 20, 21] destacamos [22, 23] que são dedicados ao estudo das integrais de trajetória.

Seja um campo de calibre $A_{\mu}^{a}$ que se transforma na representação adjunta ${ }^{1}$ do grupo de calibre $G$. A lagrangiana da teoria de Yang-Mills pura no formalismo usual para esse campo de calibre é dada por

$$
\mathcal{L}_{\mathrm{YM}}^{(2)}=-\frac{1}{4}\left(\partial_{\mu} A_{\nu}^{a}-\partial_{\nu} A_{\mu}^{a}+g f^{a b c} A_{\mu}^{b} A_{\nu}^{c}\right)^{2}
$$

\footnotetext{
${ }^{1}$ Os detalhes são dados no apêndice A. Por exemplo, lagrangiana da teria de Yang-Mills é invariante por transformações de calibre do campo $A_{\mu}^{a}$ que são dadas (de forma geral) na Eq. (A.5).
} 
onde $f^{a b c}$ são as constantes de estrutura do grupo $G$ e $g$ a constante de acoplamento. Essa é a formulação usual para a lagrangiana da teoria de YM; sua derivação pode ser encontrada no apêndice A. Note que lagrangiana da teoria de YM na Eq. (2.1) é uma generalização ${ }^{2}$ da lagrangiana da eletrodinâmica clássica, essa generalização foi considerada primeiramente por Yang e Mills [2].

As constantes de estrutura são obtidas em termo dos geradores do grupo, tal que,

$$
\left[T^{a}, T^{b}\right]=i f^{a b c} T^{c} .
$$

Se os geradores do grupo comutam $f^{a b c}=0$, o grupo é dito abeliano. Caso contrário, isso é, os geradores do grupo não comutam, o grupo é dito não abeliano. A teoria de YM é denominada de calibre não abeliana, pois o seu grupo de calibre é não abeliano. Enquanto a eletrodinâmica clássica é uma teoria de calibre abeliana, pois seu grupo de calibre, $U(1)$ (grupo das fases complexas unitárias), é um grupo abeliano.

Usualmente, o grupo de calibre $G$ é o $S U(N)$, o grupo de matrizes $N \times N$ unitárias com determinante unitário. Por exemplo, a cromodinâmica quântica e a teoria da interação fraca, ambas teorias de YM, são invariantes, respectivamente, por $S U(3)$ e $S U(2)$. Para o grupo $S U(N)$, temos $N^{2}-1$ geradores e os índices de $\operatorname{cor}^{3}$ (representados usualmente por letras latinas minusculas como $a, b, c, \ldots)$ vão de 1 até $N^{2}-1$.

Podemos obter as equações de movimento clássicas do campo de calibre $A_{\mu}^{a}$ usando as equações de Euler-Lagrange para Eq. (2.1) chegando em

$$
D_{\mu}^{a b}\left(f^{b \mu \nu}\right) \approx 0
$$

onde usamos a notação $A \approx B$ que significa: $A$ é igual a $B$, se e somente se, $A$ satisfaz sua equação de movimento clássica. Temos também a derivada covariante, na representação adjunta, definida como

$$
D_{\mu}^{a b} \equiv \delta^{a b} \partial_{\mu}+g f^{a p b} A_{\mu}^{p}
$$

e o tensor de intensidade de campo

$$
f^{a \mu \nu} \equiv \partial^{\mu} A^{a \nu}-\partial^{\nu} A^{a \mu}+g f^{a b c} A^{b \mu} A^{c \nu} .
$$

Note que a Eq. (2.1) possui termos de segunda ordem nas derivadas por essa razão denomina-se a formulação derivada por essa lagrangiana como formalismo de segunda ordem. Classicamente, tal condição não é muito relevante, mas na quantização da teoria a presença de termos de segunda ordem leva a interações mais complicadas tornando a computação de correções quânticas mais trabalhosa.

\subsubsection{Quantização}

Para a quantização das teorias de calibre não abelianas através de integrais de trajetória é necessário usar o procedimento de Faddeev-Povov [19]. Mostraremos rapidamente como podemos quantizar a teoria de YM com esse procedimento.

\footnotetext{
${ }^{2} \mathrm{Com} f^{a b c}=0$ na Eq. (2.1) obtemos o setor bosônico da lagrangiana da eletrodinâmica clássica.

${ }^{3}$ Vamos considerar genericamente como "cor" baseado na cromodinâmica quântica. No caso da teoria da interação fraca esse índice é denominado de "sabor".
} 
Devido a presença de graus de liberdade não físicos pela invariância de calibre a integral de trajetória contabiliza infinitas órbitas que no ponto de vista físico são equivalentes. Para remover essa liberdade precisamos fixar o calibre. Ao restringir a integração à uma hipersurfície que corta tais órbitas em apenas um ponto, determinadas por uma certa condição de calibre $F^{a}[A]=0$, temos em contrapartida que considerar o determinante de Faddeev-Povov $\Delta_{\mathrm{FP}}$.

Usando a relação [20, pag. 250]

$$
\Delta_{\mathrm{FP}} \equiv \operatorname{det} M=\int \mathcal{D} c^{a} \mathcal{D} \bar{c}^{b} \exp \left(-i \int d^{d} x \bar{c}^{a} M^{a b} c^{b}\right)
$$

mostramos que o determinante de $\mathrm{FP}\left(\Delta_{\mathrm{FP}}\right)$ é equivalente a introduzir dois campos de Grassmann extras $c^{a}$ e $\bar{c}^{a}$. Esses campos são conhecidos como os campos fantasmas de FP, pois são campos não físicos que não respeitam o teorema spin-estatística.

Dentro das diversas possibilidades para fixação de calibre vamos escolher a condição mais usual (leva à regras de Feynman mais simples, covariantes)

$$
F^{a}[A]=\partial^{\mu} A_{\mu}^{a}=0
$$

Para isso temos que adicionar um termo de fixação de calibre como

$$
\mathcal{L}_{\mathrm{gf}}(A)=\frac{1}{2 \xi}\left(\partial^{\mu} A_{\mu}^{a}\right)^{2}
$$

onde $\xi$ é um fator que generaliza o calibre de Lorenz. Quando $\xi \rightarrow 0$ leva ao chamado calibre de Landau que é classicamente equivalente ao calibre de Lorenz. Já quando $\xi=1$ temos o calibre de Feynman, como veremos o propagador do campo de calibre é simplificado significadamente nesse calibre. E para finalizar, da Eq. (2.6), temos o termo do setor do campo fantasma dado por

$$
\mathcal{L}_{\mathrm{gh}}(A) \equiv \bar{c}^{a} M^{a b} c^{b}=-\bar{c}^{a} \partial^{\mu} D_{\mu}^{a b} c^{b} .
$$

A derivação de $M^{a b}$ para a condição de calibre dada na Eq. (2.7) é feita em detalhes em diversos livros, como [20, eq. 7.52].

A lagrangiana (2.1) leva na lagrangiana efetiva

$$
\mathcal{L}_{\text {eff }}^{(2)}=-\frac{1}{4}\left(\partial_{\mu} A_{\nu}^{a}-\partial_{\nu} A_{\mu}^{a}+g f^{a b c} A_{\mu}^{b} A_{\nu}^{c}\right)^{2}+\frac{1}{2 \xi}\left(\partial^{\mu} A_{\mu}^{a}\right)^{2}-\bar{c}^{a} \partial^{\mu} D_{\mu}^{a b} c^{b}
$$

obtida pelo procedimento de Faddeev-Popov. Essa lagrangiana pode então ser quantizada usando o funcional gerador $Z$ das funções de Green ${ }^{4}$ que é igual a

$$
Z_{\mathrm{YM}}^{(2)}[j]=\int \mathcal{D} A_{\mu}^{a} \mathcal{D} c^{a} \mathcal{D} \bar{c}^{a} \exp i \int d^{d} x\left(\mathcal{L}_{\mathrm{eff}}^{(2)}+j^{a \mu} A_{\mu}^{a}\right),
$$

onde omitimos as fontes dos campos fantasmas (e manteremos omitindo-as por simplicidade).

Pela Eq. (2.10) vemos que há dois vértices, $(A A A)$ e $(A A A A)$, de autointeração do campo de calibre. Note que o vértice cúbico possui dependência no momento já que o termo de interação cúbico na Eq. (2.10) possui derivadas.

${ }^{4}$ Detalhes sobre o funcional gerador $Z$ e como ele gera as funções de Green são encontrados no apêndice B. Note que definimos o funcional gerador já normalizado $Z[0]=1$, onde $Z[0]$ é o funcional gerador $Z$ com fontes nulas. 


\subsubsection{Regras de Feynman}

Nessa seção listamos as regras de Feynman (os momentos entrando nos vértices) para o formalismo de segunda ordem que foram derivada no apêndice C:
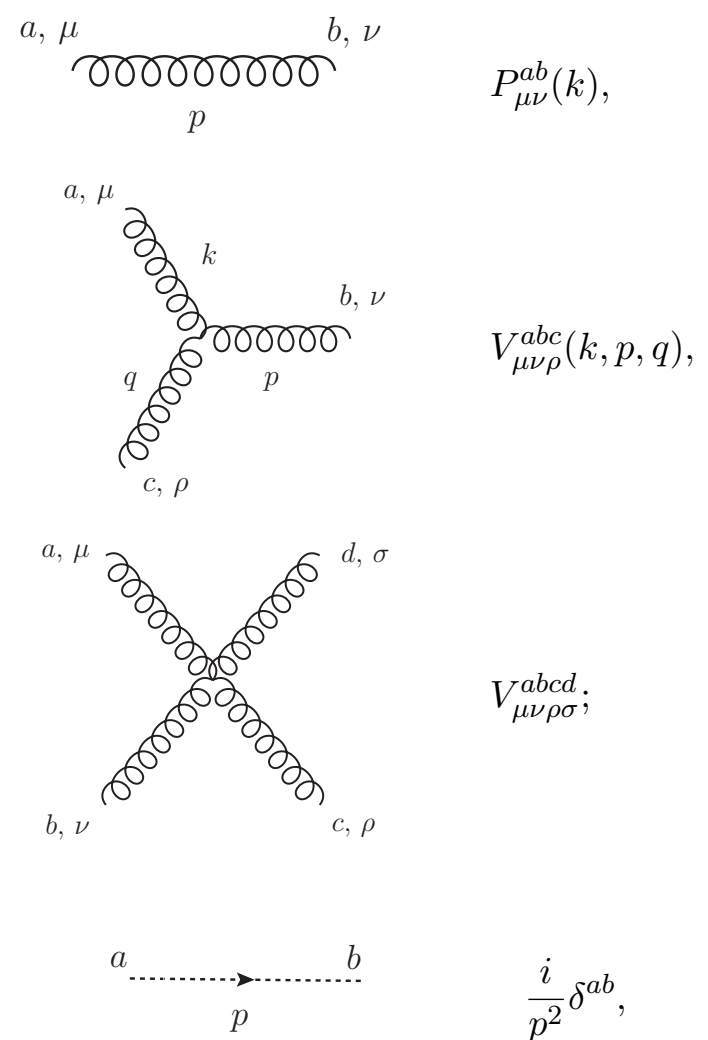

$$
\frac{i}{p^{2}} \delta^{a b}
$$

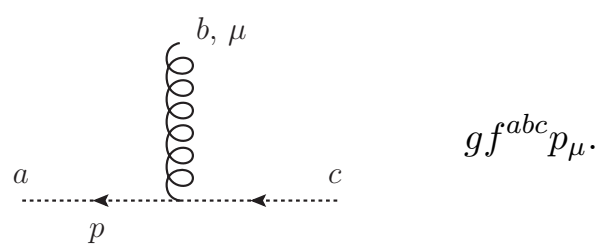

Molas representam o campo de calibre $A_{\mu}^{a}$, linhas pontilhadas representam os campos fantasmas $c^{a}, \bar{c}^{a}$. As definição explícitas de $P_{\mu \nu}^{a b}(k), V_{\mu \nu \rho}^{a b c}(k, p, q)$ e $V_{\mu \nu \rho \sigma}^{a b c d}$ são dadas respectivamente nas Eqs. (C.17), (C.23) e (C.29).

\subsection{Formalismo de primeira ordem}

Além do formalismo de segunda ordem podemos também considerar outras formulações como o formalismo de primeira ordem [4, pag. 59] onde somente temos no máximo uma dependência de primeira ordem nas derivadas.

A lagrangiana de YM no formalismo primeira ordem é dada por [5]

$$
\mathcal{L}_{\mathrm{YM}}^{(1)}=\frac{1}{4} F^{a \mu \nu} F_{\mu \nu}^{a}-\frac{1}{2} F^{a \mu \nu}\left(\partial_{\mu} A_{\nu}^{a}-\partial_{\nu} A_{\mu}^{a}+g f^{a b c} A_{\mu}^{b} A_{\nu}^{c}\right),
$$

onde temos a introdução de um campo auxiliar $F^{a \mu \nu}$. Alternativamente, podemos ver que a lagrangiana do formalismo de primeira ordem se origina da lagrangiana de YM no formalismo de 
segunda ordem

$$
\mathcal{L}_{\mathrm{YM}}^{(2)}=\frac{1}{4} f^{a \mu \nu} f_{\mu \nu}^{a}-\frac{1}{2} f^{a \mu \nu}\left(\partial_{\mu} A_{\nu}^{a}-\partial_{\nu} A_{\mu}^{a}+g f^{a b c} A_{\mu}^{b} A_{\nu}^{c}\right)
$$

tratando o tensor intensidade de campo $f_{\mu \nu}^{a}$ e o campo de calibre $A_{\mu}^{a}$ como campos independentes. Pela Eq. (2.15) já fica evidente que a lagrangiana no formalismo de primeira ordem é equivalente a lagrangiana de segunda ordem no nível clássico.

Porém antes de seguir com a quantização dessa lagrangiana, mostremos a equivalência no nível clássico de outra forma. A equação de movimento do campo auxiliar obtida a partir das equações de Euler-Lagrange é dada por

$$
F_{\mu \nu}^{a} \approx \partial_{\mu} A_{\nu}^{a}-\partial_{\nu} A_{\mu}^{a}+g f^{a b c} A_{\mu}^{b} A_{\nu}^{c}
$$

mostrando, sem surpresas, que campo auxiliar $F_{\mu \nu}^{a}$ é classicamente igual ao tensor intensidade de campo $f_{\mu \nu}^{a}$. Substituindo a Eq. (2.16) na Eq. (2.14) conclui-se que

$$
\mathcal{L}_{\mathrm{YM}}^{(1)} \approx \mathcal{L}_{\mathrm{YM}}^{(2)}
$$

Assim verifica-se que a lagrangiana de YM no formalismo de primeira ordem dada pela Eq. (2.14) é classicamente equivalente a lagrangiana de YM no formalismo de segunda ordem dada na Eq. (2.1); conforme já havíamos antecipado ao considerar a forma alternativa dada na Eq. (2.15).

Ressaltamos que a equivalência é obtida quando o campo auxiliar satisfaz sua equação de movimento clássica (2.16). Essa é uma propriedade fundamental que usaremos mais tarde para obter um método geral que demonstra a equivalência, com fontes, no âmbito quântico.

\subsubsection{Quantização}

A quantização da lagrangiana de YM no formalismo de primeiro ordem segue da mesma forma ${ }^{5}$ que no formalismo de segunda ordem. Sendo que o procedimento de Faddeev-Popov é o mesmo, uma vez que o determinante de FP não depende do campo auxiliar, o setor do campo fantasma não é alterado no formalismo de primeira ordem.

Logo, o funcional gerador $Z$ das funções de Green no formalismo de primeira ordem é dado por $^{6}$

$$
Z_{\mathrm{YM}}^{(1)}[j, J]=\int \mathcal{D} A_{\mu}^{a} \mathcal{D} F_{\mu \nu}^{a} \mathcal{D} c^{a} \mathcal{D} \bar{c}^{a} \exp i \int d^{d} x\left(\mathcal{L}_{\mathrm{YM}}^{(1)}+\mathcal{L}_{\mathrm{gf}}(A)+\mathcal{L}_{\mathrm{gh}}(A)+j^{a \mu} A_{\mu}^{a}+J^{a \mu \nu} F_{\mu \nu}^{a}\right) .
$$

A partir de Eq. (2.14) vemos que agora só há um simples vértice, $(F A A)$, sem a presença de derivadas. Porém, além dos propagadores do campo de calibre $A_{\mu}^{a}$ e do campo auxiliar $F_{\mu \nu}^{a}$ também temos propagadores mistos $\langle F A\rangle$ e $\langle A F\rangle$.

\footnotetext{
${ }^{5}$ Note que a lagrangiana do formalismo de primeira ordem é invariante pela mesma transformação de calibre do formalismo usual. Temos que, o campo de calibre se transforma da mesma forma dada pela Eq. (A.5) e o campo auxiliar $\mathcal{F}_{\mu \nu}$ se transforma como sua configuração clássica, isso é, o tensor intensidade de campo. Ou seja, $\delta \mathcal{F}_{\mu \nu}=g \mathcal{F}_{\mu \nu} g^{-1}$.

${ }^{6}$ Note que a fonte $J^{a \mu \nu}$ para o campo auxiliar $F_{\mu \nu}^{a}$ deve ser antissimétrica como o próprio campo. Para ver isso, considere uma fonte genérica $K_{\mu \nu}^{a}$, o produto $K_{\mu \nu}^{a} F^{a \mu \nu}$ deve resultar (pela antissimetria do campo $\left.F^{a \mu \nu}\right)$ em $\left(K_{-}\right)_{\mu \nu}^{a} F^{a \mu \nu}$, onde $K_{-}$é a parte assimétrica da fonte $K$. Portanto, podemos assumir a princípio que $K_{\mu \nu}^{a}=-K_{\nu \mu}^{a}$ demostrando o caráter assimétrico da fonte $J_{\mu \nu}^{a}$.
} 
A teoria perturbativa do formalismo de primeira ordem é bem mais simples do que no caso do formalismo usual, pois há somente um vértice cúbico independente de momento. ${ }^{7}$ Embora o formalismo de primeira ordem leve a uma simplificação nos vértices da teoria de YM, do outro lado temos a presença de propagadores mistos.

Tais propagadores, não tão comuns, levam a algumas dificuldades, como a obtenção de amplitudes e diagramas $1 \mathrm{PI}^{8}$ (one particle irreductible). Verificamos explicitamente (o código é dado no apêndice F) que a amplitude do espalhamento glúon-glúon no nível árvore é a mesma em ambos formalismos. Nota-se que o formalismo de primeira ordem leva a uma reorganização das contribuições, onde uma contribuição complicada no formalismo usual é igual a uma soma de contribuições mais simples no formalismo de primeira ordem.

No artigo [24] encontra-se uma generalização da fórmula de redução Lehmann-SymanzikZimmermann (LSZ) (necessária para obtenção de amplitudes) quando temos a presença de propagadores mistos. A abordagem para obter as amplitudes off-shell ${ }^{9}$ usada para verificar a equivalência da amplitude do espalhamento glúon-glúon é semelhante ao método encontrado nesse artigo, porém não consideramos a fórmula de redução LSZ completa, uma vez que, as amplitudes off-shell são mais gerais ${ }^{10}$. Além disso, as dificuldades para definir a ação efetiva (e em consequência os diagramas 1PI gerados pela ação efetiva) pelo acoplamento dos campos (isso é, propagadores mistos não nulos) podem ser contornadas pela abordagem encontrada no artigo [25]. Contudo, veremos a seguir que existe uma formulação derivada do formalismo de primeira ordem onde não há a presença de propagadores mistos [6]. Dessa forma não encontramos mais tais dificuldades e também veremos que esse formalismo "diagonal" ainda mantêm parte da simplificação proporcionada pela formulação de primeira ordem.

\subsubsection{Regras de Feynman}

Nessa seção listamos as regras de Feynman restantes (as demais são iguais a do formalismo de segunda ordem) para o formalismo de primeira ordem derivadas no apêndice C:

\footnotetext{
${ }^{7}$ Além disso, é interessante mencionar que a estrutura da lagrangiana da teoria de YM no formalismo de primeira ordem é mais apropriada para a quantização canônica [4]. Embora a quantização via integral de trajetória venha tomando um grande espaço nas últimas décadas devido a sua simplicidade e objetividade na computação das funções de Green, a quantização canônica é teoricamente mais relevante para entendemos os componentes de uma teoria física. Uma interpretação física para o campo auxiliar pela sua quantização via integral de trajetória é muito mais difícil de se obter do que através da sua quantização canônica. Embora a análise via quantização canônica feita na Ref. [4] não seja muito esclarecedora revela-se que o campo auxiliar leva a uma densidade Hamiltoniana positiva-definida [4, pág. 63].

${ }^{8}$ Para detalhes, ver apêndice B.3.

${ }^{9} \mathrm{O}$ termo on-shell se refere a "camada de massa", isso é, a relação massa-energia $E^{2}=p^{2}+m^{2}$ $\left(p^{\mu} p_{\mu}=m^{2}\right)$ é satisfeita. Por "amplitudes off-shell" designamos amplitudes cuja partículas externas não estão na camada de massa e cuja polarização não foi considerada.

${ }^{10}$ Não consideramos que os campos externos (glúons não virtuais) estão na camada de massa e a transversalidade da polarização dos estados finais.
} 


$$
a, \mu
$$

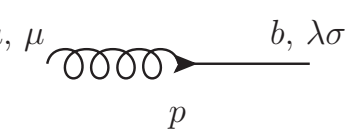

$$
-\frac{p_{\lambda} \eta_{\sigma \mu}-p_{\sigma} \eta_{\lambda \mu}}{p^{2}} \delta^{a b}
$$

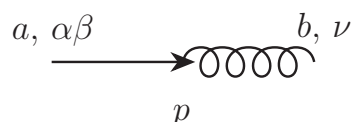

$\frac{p_{\alpha} \eta_{\beta \nu}-p_{\beta} \eta_{\alpha \nu}}{p^{2}} \delta^{a b}$

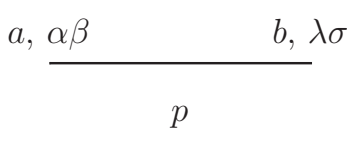

$$
2 i\left(I_{\alpha \beta \lambda \sigma}+L_{\alpha \beta \lambda \sigma}\right) \delta^{a b}
$$

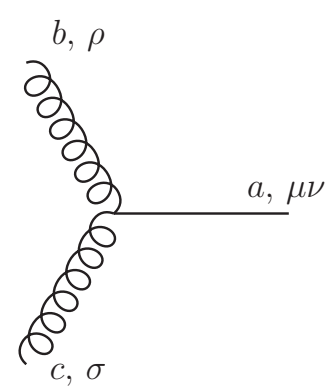

onde $\mathcal{V}_{\mu \nu \rho \sigma}^{a b c}$ é definido na Eq. (C.42), os tensores $I$ e $L$ são definidos, respectivamente, nas Eqs. (3.40) e (C.40). Nesse caso, a linha sólida representa o campo auxiliar $F_{\mu \nu}^{a}$.

\section{$2.3 \quad$ Formalismo diagonal}

Nessa seção estudaremos a diagonalização dos propagadores do formalismo de primeira ordem da teoria de YM. Veremos que mesmo removendo os propagadores mistos ainda mantemos algumas das propriedades do formalismo de primeira ordem, tal que, o formalismo diagonal ainda pode ser considerado uma boa alternativa para o estudo perturbativo da teoria de YM.

Conforme [6] podemos considerar alternativamente a lagrangiana

$$
\mathcal{L}_{\mathrm{YM}}^{(1 d)}=\frac{1}{4} H^{a \mu \nu} H_{\mu \nu}^{a}-\frac{1}{2}\left(H^{a \mu \nu}+\partial^{\mu} A^{a \nu}-\partial^{\nu} A^{a \mu}\right)\left(g f^{a b c} A_{\mu}^{b} A_{\nu}^{c}\right)-\frac{1}{4}\left(\partial_{\mu} A_{\nu}^{a}-\partial_{\nu} A_{\mu}^{a}\right)^{2}
$$

onde temos um novo campo auxiliar $H_{\mu \nu}^{a}$. A lagrangiana acima é obtida a partir da lagrangiana dada na Eq. (2.14) usando a relação clássica

$$
F_{\mu \nu}^{a} \approx \partial_{\mu} A_{\nu}^{a}-\partial_{\nu} A_{\mu}^{a}+H_{\mu \nu}^{a}
$$

que aparecerá quanticamente como um shift, como na Ref. [6, eq. 2.6], no funcional gerador dado na Eq. (2.18).

Como podemos ver a lagrangiana dada na Eq. (2.20) não apresenta termos bilineares mistos, ou seja, os campos não estão mais acoplados como na Eq. (2.14). Assim não há mais propagadores mistos (devido a presença de elementos não diagonais) e por essa razão tal formulação é denominada como formalismo diagonal. ${ }^{11}$

\footnotetext{
${ }^{11} \mathrm{O}$ termo foi cunhado no artigo [9], onde encontra-se a renormalização da teoria de YM nesse mesmo formalismo.
} 
Como vimos para o formalismo de primeira ordem, a equivalência clássica é verificada quando o campo auxiliar está na sua configuração clássica que no caso do formalismo diagonal é dada por

$$
H_{\mu \nu}^{a} \approx g f^{a b c} A_{\mu}^{b} A_{\nu}^{c} \equiv h_{\mu \nu}^{a}
$$

onde $h_{\mu \nu}^{a}$ é análogo a $f_{\mu \nu}^{a}$. Substituindo a Eq. (2.22) na lagrangiana do formalismo diagonal, temos que

$$
\mathcal{L}_{\mathrm{YM}}^{(1 d)} \approx \mathcal{L}_{\mathrm{YM}}^{(2)}
$$

mostrando a equivalência clássica dos formalismos diagonal e de segunda ordem.

A quantização da lagrangiana de YM nesse formalismo é igual a quantização estudada na formulação de primeira ordem já que estão relacionados por um shift. Portanto, diretamente da Eq. (2.18), definimos o funcional gerador das funções de Green na formulação diagonal por

$$
Z_{\mathrm{YM}}^{(1 d)}[j, J]=\int \mathcal{D} A_{\mu}^{a} \mathcal{D} H_{\mu \nu}^{a} \mathcal{D} c^{a} \mathcal{D} \bar{c}^{a} \exp i \int d^{d} x\left(\mathcal{L}_{\mathrm{YM}}^{(1 d)}+\mathcal{L}_{\mathrm{gf}}(A)+\mathcal{L}_{\mathrm{gh}}(A)+j^{a \mu} A_{\mu}^{a}+J^{a \mu \nu} H_{\mu \nu}^{a}\right)
$$

A partir de Eq. (2.20) vemos que além do vértice $(H A A)$, igual ao vértice $(F A A)$ da formulação de primeira ordem, temos o vértice cúbico $(A A A)$, o mesmo vértice da formulação de segunda ordem. ${ }^{12}$ No entanto, só temos os propagadores do campo de calibre $A_{\mu}^{a}$ e do campo auxiliar $H_{\mu \nu}^{a}$, não há propagadores mistos.

Também vemos que nesse formalismo temos termos que dependem até segunda ordem nas derivadas. Ou seja, o formalismo não é de "primeira ordem" embora seja derivado dele. O formalismo diagonal da teoria de YM pode ser visto mais como um modelo para o formalismo análogo encontrado para gravitação onde tem um papel mais relevante já que a simplificação na gravitação é muito maior que no caso da teoria de YM. Na teoria de YM um vértice quártico se reduz a um vértice cúbico, enquanto na gravitação infinitos vértices (ordem quártica e superior) se reduzem a dois vértices cúbicos.

\subsubsection{Regras de Feynman}

Nessa seção listamos as regras de Feynman (momentos entrando) restante para o formalismo diagonal que foram derivadas no apêndice $\mathrm{C}$ :

$$
\underline{\underline{\mu \nu} \quad b, \alpha \beta} \quad 2 i I_{\mu \nu \alpha \beta} \delta^{a b}
$$

\footnotetext{
${ }^{12}$ Essas equivalências não são coincidências. Usando a equivalência quântica podemos mostrar que as amplitudes (inclusive off-shell) para o campo de calibre são as mesmas em todos os formalismos. Além disso, as amplitudes off-shell dos campos auxiliares devem ser iguais da mesma forma. Isso mostraria, por exemplo, que a amplitude $(F A A)$ tem que ser a mesma que a amplitude $(H A A)$, pois os vértices são as únicas contribuições no nível árvore implicando que as respectivas regras de Feynman coincidam. Para o caso de amplitudes físicas (on-shell) basta considerar o teorema de equivalência da matriz $S$ [26]. Esse teorema afirma que a matriz $S$ (cujo elementos são as amplitudes físicas) é invariante diante redefinições locais nos campos que é o caso da redefinição do campo $F_{\mu \nu}^{a}$ ou $H_{\mu \nu}^{a}$ dada na Eq. (2.21).
} 


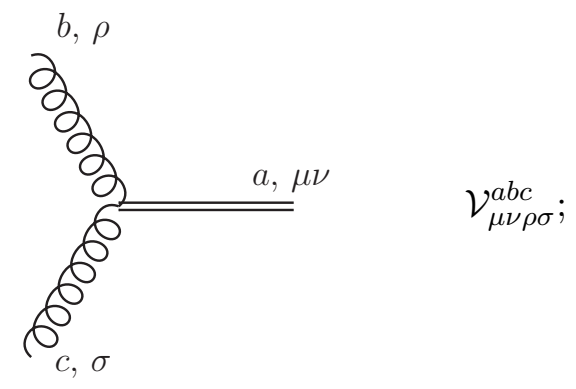

onde $I_{\mu \nu \alpha \beta}$ é o tensor identidade definido na Eq. (3.40). As linhas duplas representam o campo auxiliar $H_{\mu \nu}^{a}$. 



\section{CAPÍTULO 3}

\section{EquivalênCIA QuÂNTICA: TEORIA DE YANG-MILLS}

Neste capítulo demonstraremos a equivalência quântica das formulações da teoria de YM estudadas no capítulo anterior. Além disso, derivaremos identidades entre funções de Green computadas no formulações de primeira ordem ou diagonal com correlações calculadas no formalismo de segunda ordem. Esse capítulo, em sua maioria, é inspirado nos artigos $[15,16]$ publicados $^{1}$ durante esse trabalho.

Demostraremos que o formalismo de primeira ordem e o formalismo diagonal são equivalentes no nível quântico ao formalismo de segunda ordem implicando a equivalência quântica de todas as formulações consideradas. Primeiramente, vamos demostrar a equivalência quântica, sem fontes, para o formalismo de primeira ordem conforme visto nas Ref. [4, 5]. E então mostraremos que podemos inferir uma condição geral (como ansatz) para obter a equivalência quântica concordando com a abordagem dos artigos $[15,16]$.

\subsection{Equivalência parcial para o formalismo de pri- meira ordem}

Nessa seção vamos mostrar que o funcional gerador $Z$, sem fontes, no formalismo de primeira e de segunda ordem são iguais. Essa igualdade é o que denominamos de equivalência parcial, a parcialidade está no fato que não consideramos as fontes que possuem um papel essencial na obtenção das funções de Green. Não é claro se a equivalência parcial é suficiente para garantir a equivalência com fontes, embora seja necessária. Além disso, ao mostrar a equivalência parcial podemos ver que tal procedimento pode ser generalizado inferindo uma condição que pode também englobar o caso com fontes.

\footnotetext{
${ }^{1}$ Minha maior contribuição foi na verificação dos cálculos explícitos em um loop que são detalhados no capítulo 6 e nos apêndices D e E.
} 
Partindo do funcional gerador do formalismo de primeira ordem sem fontes ${ }^{2}$

$$
Z_{\mathrm{YM}}^{(1)}[0] \equiv Z_{\mathrm{YM}}^{(1)}[0,0]=\int \mathcal{D} A_{\mu}^{a} \mathcal{D} F_{\mu \nu}^{a} \exp i \int d^{d} x\left(\mathcal{L}_{\mathrm{YM}}^{(1)}+\mathcal{L}_{\mathrm{gf}}(A)+\mathcal{L}_{\mathrm{gh}}(A)\right)
$$

podemos fazer o shift ${ }^{3}$ no campo auxiliar $F_{\mu \nu}^{a}$

$$
F_{\mu \nu}^{a}=\tilde{F}_{\mu \nu}^{a}+\partial_{\mu} A_{\nu}^{a}-\partial_{\nu} A_{\mu}^{a}+g f^{a b c} A_{\mu}^{b} A_{\nu}^{c}
$$

mantendo o funcional gerador invariante, pois a medida é invariante $\mathcal{D} F^{a \mu \nu}=\mathcal{D} \tilde{F}^{a \mu \nu}$. A única parte que deve ser alterada com o shift é a lagrangiana de primeira ordem, consideremos somente ela. Aplicando o shift (3.2) na Eq. (2.14):

$$
\begin{aligned}
\tilde{\mathcal{L}}_{\mathrm{YM}}^{(1)} & =\frac{1}{4}\left(\tilde{F}_{\mu \nu}^{a}+\partial_{\mu} A_{\nu}^{a}-\partial_{\nu} A_{\mu}^{a}+g f^{a b c} A_{\mu}^{b} A_{\nu}^{c}\right)^{2}-\frac{1}{2}\left(\tilde{F}_{\mu \nu}^{a}+\partial_{\mu} A_{\nu}^{a}-\partial_{\nu} A_{\mu}^{a}+g f^{a b c} A_{\mu}^{b} A_{\nu}^{c}\right) f^{a \mu \nu} \\
& =\frac{1}{4} \tilde{F}_{\mu \nu}^{a} \tilde{F}^{a \mu \nu}+\frac{1}{4}\left(\partial_{\mu} A_{\nu}^{a}-\partial_{\nu} A_{\mu}^{a}+g f^{a b c} A_{\mu}^{b} A_{\nu}^{c}\right)^{2}-\frac{1}{2}\left(\partial_{\mu} A_{\nu}^{a}-\partial_{\nu} A_{\mu}^{a}+g f^{a b c} A_{\mu}^{b} A_{\nu}^{c}\right) f^{a \mu \nu} \\
& =\frac{1}{4} \tilde{F}_{\mu \nu}^{a} \tilde{F}^{a \mu \nu}-\frac{1}{2}\left(\partial_{\mu} A_{\nu}^{a}-\partial_{\nu} A_{\mu}^{a}+g f^{a b c} A_{\mu}^{b} A_{\nu}^{c}\right)^{2}=\frac{1}{4} \tilde{F}_{\mu \nu}^{a} \tilde{F}^{a \mu \nu}+\mathcal{L}_{\mathrm{YM}}^{(2)}
\end{aligned}
$$

Usando o resultado obtido acima temos que o funcional gerador da Eq. (3.1) após o shift (3.2) é igual a

$$
\begin{aligned}
Z_{\mathrm{YM}}^{(1)}[0] & =\int \mathcal{D} \tilde{F}_{\mu \nu}^{a} \exp i \int d^{d} x\left(\frac{1}{4} \tilde{F}_{\mu \nu}^{a} \tilde{F}^{a \mu \nu}\right) \\
& \times \int \mathcal{D} A_{\mu}^{a} \mathcal{D} c^{a} \mathcal{D} \bar{c}^{a} \exp i \int d^{d} x\left(\mathcal{L}_{\mathrm{YM}}^{(2)}+\mathcal{L}_{\mathrm{gf}}(A)+\mathcal{L}_{\mathrm{gh}}(A)\right)
\end{aligned}
$$

A primeira integral de trajetória na Eq. (3.4) é uma constante que pode ser absorvida pela normalização do funcional gerador e por isso podemos descartá-la. Já a segunda integral é igual ao funcional gerador do formalismo de segunda ordem sem fontes (2.11), tal que podemos concluir que

$$
Z_{\mathrm{YM}}^{(1)}[0]=Z_{\mathrm{YM}}^{(2)}[0]
$$

mostrando a equivalência quântica sem fontes.

Para generalizar o resultado da Eq. (3.5) para o caso com presença de fontes mostraremos como encontrar o shift do caso anterior através de argumentos clássicos. Podemos reescrever o shift (3.2) de forma sugestiva como

$$
F_{\mu \nu}^{a}=\tilde{F}_{\mu \nu}^{a}+f_{\mu \nu}^{a},
$$

\footnotetext{
${ }^{2}$ Note que aqui estamos considerando que o funcional gerador $Z$ não é normalizado, $Z[0] \neq 1$, diferentemente do apêndice B. A normalização pode ser considerada sem inconsistências quando estamos trabalhando com um único funcional gerador, mas quando temos diferentes funcionais geradores é claro que $Z[0]$ é diferente para cada caso. Como mostraremos aqui esses funcionais geradores são iguais para $j=J=0$, logo podemos considerar a normalização sem qualquer inconsistência. Isso é, podemos considerar que $Z[0]=1$ quando consideramos o caso com as fontes.

${ }^{3} \mathrm{O}$ shift é inverso ao considerado nas Refs. $[4,5]$ já que partimos do formalismo de primeira ordem, ou seja, invertemos a origem. A demostração é mais direta por mais que seja aparentemente menos natural.
} 
onde pela Eq. (2.16) identifica-se que o shift considerado no campo auxiliar é em relação a sua configuração clássica. ${ }^{4}$ Claramente, se $\tilde{F}_{\mu \nu}^{a}=0$ temos que Eq. (3.6) é equivalente a Eq. (2.16).

Logo, a manifestação da equivalência clássica ao nível quântico se dá por um shift no campo auxiliar em relação a sua configuração clássica e segue pela a integração dos graus de liberdades do campo auxiliar que são irrelevantes do ponto de vista físico já que o campo auxiliar é não físico ${ }^{5}$. Dessa maneira, podemos considerar como ansatz que o shift necessário para demonstrar a equivalência (integrar o campo auxiliar) é obtido pela condição

$$
\tilde{F}_{\mu \nu}^{a}=F_{\mu \nu}^{a}-S_{\mu \nu}^{a} \approx 0 \Leftrightarrow F_{\mu \nu}^{a} \approx S_{\mu \nu}^{a},
$$

isso é, $S_{\mu \nu}^{a}$ é igual a configuração clássica do campo auxiliar $F_{\mu \nu}^{a}$.

Aplicaremos a condição (3.7) no caso geral com fontes para encontrar um shift válido para integração do campo auxiliar nesse caso. No artigo [16] é mencionado a relação entre o shift e a configuração clássica do campo auxiliar no caso da gravitação em analogia com o caso da teoria de YM, enquanto aqui elevamos tal relação a uma condição suficiente e necessária para obtemos a equivalência quântica através do método desenvolvido em [15, 16].

\subsection{Equivalência quântica para o formalismo de pri- meira ordem}

Agora consideraremos o caso geral com fontes. O funcional gerador do formalismo de primeira ordem com fontes é igual a

$$
Z_{\mathrm{YM}}^{(1)}[j, J]=\int \mathcal{D} A_{\mu}^{a} \mathcal{D} F_{\mu \nu}^{a} \mathcal{D} c^{a} \mathcal{D} \bar{c}^{a} \exp i \int d^{d} x\left(\mathcal{L}_{\mathrm{YM}}^{(1)}+\mathcal{L}_{\mathrm{gf}}(A)+\mathcal{L}_{\mathrm{gh}}(A)+j^{a \mu} A_{\mu}^{a}+J^{a \mu \nu} F_{\mu \nu}^{a}\right)
$$

Tentando aplicar o shift anterior (3.6) chegariamos no funcional gerador

$$
\begin{aligned}
Z_{\mathrm{YM}}^{(1)}[j, J] & =\int \mathcal{D} \tilde{F}_{\mu \nu}^{a} \exp i \int d^{d} x\left(\frac{1}{4} \tilde{F}_{\mu \nu}^{a} \tilde{F}^{a \mu \nu}+J^{a \mu \nu} \tilde{F}_{\mu \nu}^{a}\right) \\
& \times \int \mathcal{D} A_{\mu}^{a} \mathcal{D} c^{a} \mathcal{D} \bar{c}^{a} \exp i \int d^{d} x\left(\mathcal{L}_{\mathrm{YM}}^{(2)}+\mathcal{L}_{\mathrm{gf}}(A)+\mathcal{L}_{\mathrm{gh}}(A)+j^{a \mu} A_{\mu}^{a}-J^{a \mu \nu} f_{\mu \nu}^{a}\right),
\end{aligned}
$$

onde não podemos integrar diretamente o campo auxiliar $\tilde{F}_{\mu \nu}^{a}$. No entanto, podemos tentar integrar a primeira integral acima "completando o quadrado". Dessa maneira teríamos

$$
\frac{1}{4} \tilde{F}_{\mu \nu}^{a} \tilde{F}^{a \mu \nu}+J^{a \mu \nu} \tilde{F}_{\mu \nu}^{a}=\frac{1}{4}\left(\tilde{F}_{\mu \nu}^{a}+2 J_{\mu \nu}^{a}\right)^{2}-J^{a \mu \nu} J_{\mu \nu}^{a}
$$

que sugere considerar um shift

$$
\tilde{F}_{\mu \nu}^{a} \rightarrow \tilde{F}_{\mu \nu}^{a}-2 J_{\mu \nu}^{a}
$$

\footnotetext{
${ }^{4}$ Vale a pena notar que tal shift é de certa forma semelhante ao formalismo de campo de fundo [27], onde expandimos um campo $A$ um torno de um campo de fundo $B$, tal que $A=B+Q$. O campo $Q$ teria o papel do campo quântico, enquanto $B$ é arbitrário. Por exemplo, podemos escolher ele tal que satisfaça a equação de movimento do campo $A$. No formalismo de primeira ordem, teríamos que $Q=\tilde{F}_{\mu \nu}^{a}$ que como vimos pode ser integrado sem consequências da Eq. (3.4). Isso indica que o caráter quântico do campo $F_{\mu \nu}^{a}$ é irrelevante, sendo apenas um campo auxiliar como já era esperado.

${ }^{5} \mathrm{~A}$ princípio não associamos nenhuma física a esse campo, por exemplo espera-se que nenhuma partícula associada a ele realmente participe de processos físicos (contribua para a matriz $S$ ).
} 
Fazendo esse shift na Eq. (3.9) chegamos no funcional gerador

$$
\begin{aligned}
& Z_{\mathrm{YM}}^{(1)}[j, J]=\int \mathcal{D} \tilde{F}_{\mu \nu}^{a} \exp i \int d^{d} x\left(\frac{1}{4} \tilde{F}_{\mu \nu}^{a} \tilde{F}^{a \mu \nu}\right) \\
& \times \int \mathcal{D} A_{\mu}^{a} \mathcal{D} c^{a} \mathcal{D} \bar{c}^{a} \exp i \int d^{d} x\left(\mathcal{L}_{\mathrm{YM}}^{(2)}+\mathcal{L}_{\mathrm{gf}}(A)+\mathcal{L}_{\mathrm{gh}}(A)+j^{a \mu} A_{\mu}^{a}+J^{a \mu \nu} f_{\mu \nu}^{a}-J^{a \mu \nu} J_{\mu \nu}^{a}\right) .
\end{aligned}
$$

Agora a integral no campo auxiliar $\tilde{F}_{\mu \nu}^{a}$, assim como anteriormente, é uma constante que pode ser descartada e obtemos o mesmo resultado do artigo [15].

Também pode-se partir diretamente do funcional gerador do formalismo de primeira ordem considerando o shift, conforme é visto no artigo [Eq. (1.3)][15],

$$
F_{\mu \nu}^{a}=\tilde{F}_{\mu \nu}^{a}+f_{\mu \nu}^{a}-2 J_{\mu \nu}^{a}
$$

que resulta dos shifts parciais (3.6) e (3.10).

Para $J=0$, a lagrangiana efetiva que aparece no lado direito da Eq. (3.11) é igual a lagrangiana efetiva do formalismo de segunda ordem, tal que, podemos reescrever a Eq. (3.11) como

$$
Z_{\mathrm{YM}}^{(1)}[j, J]=\int \mathcal{D} A_{\mu}^{a} \mathcal{D} c^{a} \mathcal{D} \bar{c}^{a} \exp i \int d^{d} x\left(\mathcal{L}_{\mathrm{eff}}^{(2)}+j^{a \mu} A_{\mu}^{a}+J^{a \mu \nu} f_{\mu \nu}^{a}-J^{a \mu \nu} J_{\mu \nu}^{a}\right)
$$

ficando evidente a equivalência entre os funcionais geradores das Eqs. (2.11) e Eq. (2.18).

Com $J=0$ na Eq. (3.13), temos que [15]

$$
Z_{\mathrm{YM}}^{(1)}[j, 0]=Z_{\mathrm{YM}}^{(2)}[j]
$$

sendo assim, os formalismo de primeira e segunda ordem podem ser ditos equivalentes no nível quântico. Consequentemente, o formalismo de primeira ordem leva nas mesmas funções de Green com campos de calibre $A_{\mu}^{a}$ externos que o formalismo de segunda ordem:

$$
\begin{aligned}
\left\langle 0\left|T A^{a_{1} \mu_{1}}\left(x_{1}\right) \cdots A^{a_{n} \mu_{n}}\left(x_{n}\right)\right| 0\right\rangle & \left.\equiv(-i)^{n} \frac{\delta^{n} Z_{\mathrm{YM}}^{(2)}[j]}{\delta j_{\mu_{1}}^{a_{1}}\left(x_{1}\right) \cdots \delta j_{\mu_{n}}^{a_{n}}\left(x_{n}\right)}\right|_{j=0} \\
& =\left.(-i)^{n} \frac{\delta^{n} Z_{\mathrm{YM}}^{(1)}[j, J]}{\delta j_{\mu_{1}}^{a_{1}}\left(x_{1}\right) \cdots \delta j_{\mu_{n}}^{a_{n}}\left(x_{n}\right)}\right|_{J=j=0} .
\end{aligned}
$$

Em [págs. 65-67][4] mostra-se que o propagador do campo de calibre $A_{\mu}^{a}$ é igual nos dois formalismos até o nível de um loop que verifica a validade (até um loop) da Eq. (3.15) para $n=2$. Nós também verificamos explicitamente a identidade acima com $n=4$, no nível árvore, ao mostrar a equivalência da amplitude de 4-glúons. O código usado para a verificação e alguns detalhes da computação estão no apêndice F.

Antes de continuar a discussão sobre o funcional gerador obtido acima mostraremos como podemos usar a condição geral (3.7) para obter o shift (3.12). Com a presença de fontes é esperado que a configuração clássica do campo auxiliar $F_{\mu \nu}^{a}$ deva considerar tais fontes. Para isso em vez de usar a lagrangiana clássica precisamos considerar a lagrangiana efetiva ${ }^{6}$ com fontes clássicas dada por

$$
\mathcal{L}_{\text {eff }}^{(1)}=\mathcal{L}_{\mathrm{YM}}^{(1)}+\mathcal{L}_{\mathrm{gf}}(A)+\mathcal{L}_{\mathrm{gh}}(A)+j^{a \mu \nu} A_{\mu \nu}^{a}+J^{a \mu \nu} F_{\mu \nu}^{a}
$$

\footnotetext{
${ }^{6}$ No caso das equações de movimento para o campo auxiliar os termos extras da lagrangiana efetiva não são revelantes.
} 
onde o termo que depende das fontes é igual ao termo adicionado durante o procedimento de quantização via integrais de trajetória. Usando as equações de Euler-Lagrange para o campo auxiliar obtemos

$$
F_{\mu \nu}^{a} \approx f_{\mu \nu}^{a}-2 J_{\mu \nu}^{a}=S_{\mu \nu}^{a},
$$

tal que o shift, pela condição (3.7), deve ser

$$
F_{\mu \nu}^{a}=\tilde{F}_{\mu \nu}^{a}+S_{\mu \nu}^{a}=\tilde{F}_{\mu \nu}^{a}+f_{\mu \nu}^{a}-2 J_{\mu \nu}^{a} .
$$

Esse é o mesmo shift derivado acima (ver Eq. (3.12)), sendo igual ao considerado na Ref. [15]. Isso verifica o uso da condição (3.7) no caso com fontes cuja futura generalização para gravitação será muito útil.

Seguindo, para $J \neq 0$, o lado direito da Eq. (3.13) pode ser visto como uma extensão do funcional gerador do formalismo de segunda ordem dado na Eq. (2.11). Pois além de gerar funções de Green para o campo de calibre $A_{\mu}^{a}$ também gera funções de Green para o operador composto $f_{\mu \nu}^{a}(x)=\partial_{\mu} A_{\nu}^{a}(x)-\partial_{\nu} A_{\mu}^{a}(x)+g f^{a b c} A_{\mu}^{b}(x) A_{\nu}^{c}(x)$.

Embora pareça que a dependência quadrática em $J$ na Eq. (3.13) seja incomum, como mencionado no artigo [15], é fácil de ver como essa dependência aparece. Consideremos o procedimento usual para integrar um campo escalar livre $\phi(x)$ usando seu propagador $\Delta(x-y)$. Do seu funcional gerador

$$
Z[J]=\int \mathcal{D} \phi \exp i \int d^{d} x\left(-\frac{1}{2} \phi(x) \Delta^{-1}(x) \phi(x)+J \phi\right)
$$

nós fazemos o shift

$$
\phi(x)=\tilde{\phi}(x)+\int d^{d} y \Delta(x-y) J(y)
$$

tal que a Eq. (3.19) toma a forma

$$
\left.Z[J]=\int \mathcal{D} \tilde{\phi} \exp i\left[\int d^{d} x\left(-\frac{1}{2} \tilde{\phi}(x) \Delta^{-1}(x) \tilde{\phi}(x)\right)\right)\right] \exp i \int d^{d} x d^{d} y \frac{J(x) \Delta(x-y) J(y)}{2} .
$$

Veja que o primeiro termo da Eq. (3.21) é uma constante que pode ser descartada via normalização como a integral do campo $\tilde{F}_{\mu \nu}^{a}$, e assim como na Eq. (3.13) temos uma dependência quadrática em $J$. Claramente não temos um análogo para o acoplamento da fonte $J$ com o tensor intensidade de campo, pois o campo $\phi$ é livre enquanto o campo auxiliar $F_{\mu \nu}^{a}$ é acoplado linearmente com o campo de calibre $A_{\mu}^{a}$. Porém, o procedimento usual acima é suficiente para mostrar que a dependência quadrática em $J$ é esperada ao integrar o campo auxiliar $F_{\mu \nu}^{a}$.

Além disso, note que o shift (3.20) respeita a condição (3.7) já que o último termo da Eq. (3.20) não passa da solução da equação (diferencial) clássica com fontes (não homogênea) que o campo escalar satisfaz.

Ressaltamos que no caso do formalismo de primeira ordem as inversas dos termos bilineares nos campos não são iguais ao propagadores como para o campo $\phi(x)$. No formalismo de primeira ordem temos termos mistos e precisamos considerar a inversa de uma matriz. Logo, se consideramos o termo bilinear no campo auxiliar definido como

$$
-\frac{1}{2} F^{a \mu \nu} D_{\mu \nu \alpha \beta} F^{a \alpha \beta} \equiv \frac{1}{4} F^{a \mu \nu} F_{\mu \nu}^{a}
$$


obtemos que $D_{\mu \nu \alpha \beta}^{-1}=-\eta_{\mu \alpha} \eta_{\nu \beta}+\eta_{\mu \beta} \eta_{\nu \alpha}$, de forma que o último termo da Eq. (3.21) é análogo a

$$
\frac{1}{2} J^{a \mu \nu} D_{\mu \nu \alpha \beta}^{-1} J^{a \alpha \beta}=-J^{a \mu \nu} J_{\mu \nu}^{a}
$$

Chegando assim na mesma dependência em $J$ da Eq. (3.13).

A seguir consideraremos em mais detalhes o funcional gerador obtido na Eq. (3.13). Mostraremos que as funções de Green com o campo auxiliar $F_{\mu \nu}^{a}$ externo estão relacionadas com funções de Green com o campo composto $f_{\mu \nu}^{a}(x)$. Veremos que essas identidades também sugerem uma interpretação física para o campo auxiliar $F_{\mu \nu}^{a}$.

\subsubsection{Condição de consistência}

As funções de Green no formalismo de primeira ordem são geradas pelo funcional gerador $Z_{\mathrm{YM}}^{(1)}$ dado na Eq. (2.18), alternativamente também podemos considerar a forma obtida na Eq. (3.13):

$$
Z_{\mathrm{YM}}^{(1)}[j, J]=\int \mathcal{D} A_{\mu}^{a} \mathcal{D} c^{a} \mathcal{D} \bar{c}^{a} \exp i\left[S_{\mathrm{eff}}^{(2)}+\int d^{d} x\left(j^{a \mu} A_{\mu}^{a}+J^{a \mu \nu} f_{\mu \nu}^{a}-J^{a \mu \nu} J_{\mu \nu}^{a}\right)\right]
$$

onde definimos a ação efetiva

$$
S_{\text {eff }}^{(2)} \equiv \int d^{d} x \mathcal{L}_{\text {eff }}^{(2)}
$$

No entanto, as funções de Green obtidas a partir do funcional gerador no lado direito da Eq. (3.24) são computadas no formalismo de segunda ordem, enquanto no lado esquerdo no formalismo de primeira ordem. Logo, para o formalismo de primeira ordem ser consistente certas identidades devem ser satisfeitas relacionando funções de Green computadas no formalismo de primeira ordem com funções de Green obtidas no formalismo de segunda ordem. A mais relevante delas já foi derivada, a Eq. (3.15), demostrando que as funções de Green de n-pontos do campo de calibre $A_{\mu}^{a}$ são iguais nos dois formalismos.

Já o caso das funções de Green com a presença de campos auxiliares $F_{\mu \nu}^{a}$ externos é mais elaborado. Partindo das derivadas funcionais do funcional gerador do formalismo de primeira ordem dado na Eq. (2.18) em relação a fonte do campo auxiliar $F_{\mu \nu}^{a}$ obtemos as funções de Green de $n$-pontos (ver a Eq. (B.6))

$$
\left\langle 0\left|T F^{a_{1} \mu_{1} \nu_{1}}\left(x_{1}\right) \cdots F^{a_{n} \mu_{n} \nu_{n}}\left(x_{n}\right)\right| 0\right\rangle=\left.(-i)^{n} \frac{\delta^{n} Z_{\mathrm{YM}}^{(1)}[j, J]}{\delta J_{\mu_{1} \nu_{1}}^{a_{1}}\left(x_{1}\right) \cdots \delta J_{\mu_{n} \nu_{n}}^{a_{n}}\left(x_{n}\right)}\right|_{J=j=0} .
$$

Enquanto a derivação funcional da Eq. (3.24) em relação a fonte $J_{\mu \nu}^{a}(x)$ leva a correlações (na presença de fontes) do operador $f^{a \mu \nu}(x)-2 J^{a \mu \nu}(x)$. Para ver isso considere a função de um ponto:

$$
\begin{aligned}
\frac{1}{i} \frac{\delta Z_{\mathrm{YM}}^{(1)}[j, J]}{\delta J_{\mu \nu}^{a}(x)} & =\frac{1}{i} \frac{\delta}{\delta J_{\mu \nu}^{a}(x)} \int \mathcal{D} A_{\mu}^{a} \mathcal{D} c^{a} \mathcal{D} \bar{c}^{a} \exp i\left[S_{\mathrm{eff}}^{(2)}+\int d^{d} x\left(j^{a \mu} A_{\mu}^{a}+J^{a \mu \nu} f_{\mu \nu}^{a}-J^{a \mu \nu} J_{\mu \nu}^{a}\right)\right] \\
& =\int \mathcal{D} A_{\mu}^{a} \mathcal{D} c^{a} \mathcal{D} \bar{c}^{a}\left[f^{a \mu \nu}(x)-2 J^{a \mu \nu}(x)\right] \exp i S^{(1,2)} \\
& \equiv\left\langle 0\left|T\left[f^{a \mu \nu}(x)-2 J^{a \mu \nu}(x)\right]\right| 0\right\rangle_{J},
\end{aligned}
$$


onde

$$
S^{(1,2)} \equiv S_{\mathrm{eff}}^{(2)}+\int d^{d} x\left(j^{a \mu} A_{\mu}^{a}+J^{a \mu \nu} f_{\mu \nu}^{a}-J^{a \mu \nu} J_{\mu \nu}^{a}\right)
$$

Por razão dessa dependência não usual com a fonte $J$ ao derivar as correlações do funcional gerador na sua forma alternativa (3.24) é necessário tomar cuidado com os termos devido a fonte $J$, como fizemos para deduzir a Eq. (3.27).

No limite em que as fontes se anulam, em especial $J=0$, a correlação acima passa a ser igual ao valor esperado no vácuo do tensor de intensidade de campo $f^{a \mu \nu}(x)$ :

$$
\left\langle 0\left|T\left[f^{a \mu \nu}(x)-2 J^{a \mu \nu}(x)\right]\right| 0\right\rangle_{J=0}=\left\langle 0\left|T f^{a \mu \nu}(x)\right| 0\right\rangle,
$$

por sua definição $(2.5)$

$$
f^{a \mu \nu}(x)=\partial^{\mu} A^{a \nu}(x)-\partial^{\nu} A^{a \mu}(x)+g f^{a b c} A^{b \mu}(x) A^{c \nu}(x),
$$

tal que, o seu valor esperado no vácuo é dado por funções de Green computadas no formalismo de segunda ordem. Em especial, o último termo é um exemplo de um campo composto. Um campo composto [17] pode ser definido como o produto de dois ou mais campos no mesmo ponto do espaço tempo; como é o caso do termo $g f^{a b c} A^{b \mu}(x) A^{c \nu}(x)$.

Usando a Eq. (3.26) na Eq. (3.27) $\operatorname{com} j=J=0$ obtemos a identidade

$$
\left\langle 0\left|T F^{a \mu \nu}(x)\right| 0\right\rangle=\left\langle 0\left|T f^{a \mu \nu}(x)\right| 0\right\rangle,
$$

onde o lado esquerdo é computado no formalismo de primeira ordem, enquanto o lado direito no formalismo de segunda ordem. Esse é mais um exemplo de identidade consequente da condição de consistência para os funcionais geradores do formalismo de primeira ordem; uma identidade não trivial que relaciona uma função de Green do formalismo de primeira com uma correlação computada no formalismo de segunda ordem.

Contudo, pela Eq. (3.15), também podemos computar o lado esquerdo no formalismo de primeira ordem. Assim elevamos a identidade acima para

$$
\left\langle 0\left|T F^{a \mu \nu}(x)\right| 0\right\rangle=\left\langle 0\left|T f^{a \mu \nu}(x)\right| 0\right\rangle \quad \text { (no formalismo de primeira ordem). }
$$

Essa identidade pode ser vista como uma generalização quântica da equação de movimento do campo auxiliar $F_{\mu \nu}^{a}$, i.e. $F^{a \mu \nu} \approx f^{a \mu \nu}$.

A Eq. (3.31) é um exemplo simples das identidades que podem ser obtidas como consequência da Eq. (3.13) que foi derivada ao demonstrar a equivalência dos formalismos de primeira e segunda ordem. A seguir deduziremos outras identidades semelhantes. Essas identidades estruturais [15] relacionam as funções de Green do formalismo de primeira ordem com funções de Green computadas no formalismo de segunda ordem.

\subsubsection{Identidades estruturais}

Nessa seção partiremos para a dedução de identidades estruturais mais elaboradas. Conforme fizemos na seção anterior computaremos derivadas funcionais do funcional gerador do formalismo de primeira ordem tanto na sua forma usual (2.18) quanto na forma alternativa (3.24). As 
identidades estruturais são derivadas quando consideramos as fontes $j=J=0$ obtendo uma igualdade entre as correlações dos campos considerados.

\section{FunÇÕes DE GREen DE 2-PONTOS}

Derivando a Eq. (2.18) em relação as fontes $J_{\mu \nu}^{a}(x)$ e $j_{\rho}^{b}(y)$ obtemos a função de Green mista com um campo auxiliar $F^{a \mu \nu}$ e um campo de calibre $A^{b \rho}$ externo:

$$
\left.(-i)^{2} \frac{\delta^{2} Z_{\mathrm{YM}}^{(1)}[j, J]}{\delta J_{\mu \nu}^{a}(x) \delta j_{\rho}^{b}(y)}\right|_{j=J=0}=\left\langle 0\left|T F^{a \mu \nu}(x) A^{b \rho}(y)\right| 0\right\rangle .
$$

Já para a forma alternativa (3.24) obtemos

$$
\left\langle 0\left|T\left[f^{a \mu \nu}(x)-2 J^{a \mu \nu}(x)\right] A^{b \rho}(y)\right| 0\right\rangle_{J} .
$$

Como visto antes a derivada funcional em relação a $J$ leva a uma correlação que depende a fonte $J$.

Fazendo $j=J=0$ podemos igualar as Eqs. (3.33) e (3.34), tal que deduzimos que

$$
\left\langle 0\left|T F^{a \mu \nu}(x) A^{b \rho}(y)\right| 0\right\rangle=\left\langle 0\left|T f^{a \mu \nu}(x) A^{b \rho}(y)\right| 0\right\rangle .
$$

A identidade estrutural acima relaciona o propagador completo misto do formalismo de primeira ordem $\left\langle 0\left|T F^{a \mu \nu}(x) A^{b \rho}(y)\right| 0\right\rangle$ com a correlação $\left\langle 0\left|T f^{a \mu \nu}(x) A^{b \rho}(y)\right| 0\right\rangle$ computada, a princípio, no formalismo de segunda ordem. Conforme [15] essa identidade pode ser vista como uma generalização quântica da igualdade clássica $F^{a \mu \nu} \approx f^{a \mu \nu}$.

Como mencionado no caso da Eq. (3.31), a equivalência (3.15) permite que computemos qualquer função de Green que contêm somente campos de calibre externos em ambos formalismo, o mesmo não é válido para o campo auxiliar $F_{\mu \nu}^{a}$. Porém, podemos deduzir identidades estruturais que nos permitem obter qualquer função de Green do formalismo de primeira ordem a partir de correlações computadas no formalismo de segunda ordem.

No entanto, é no caso oposto que temos uma aplicação prática mais interessante já que as identidades estruturais nos permitem obter correlações contendo campos compostos não usuais a partir das funções de Green do formalismo de primeira ordem. As funções de Green no formalismo de primeira são obtidas diretamente ${ }^{7}$ através dos diagramas de Feynman, enquanto os diagramas relativos as correlações com campos compostos são computados através da análise ${ }^{8}$ do surgimento de singularidades devidas a efeitos de curta distância [15, 17, 28]. Veremos como computar tais correlações ao verificar explicitamente algumas das identidades estruturais derivadas nesse trabalho.

Agora só resta a função de Green de 2-pontos para o campo auxiliar $F_{\mu \nu}^{a}$. Da Eq. (3.26), com $n=2$, temos a função de Green

$$
\left\langle 0\left|T F^{a \mu \nu}(x) F^{b \rho \sigma}(y)\right| 0\right\rangle .
$$

${ }^{7}$ Além de serem mais simples devido a simplificação dos cálculos perturbativos no formalismo de primeira ordem.

${ }^{8}$ Mostraremos uma forma de estender as Regras de Feynman que permitirá obter diretamente, de forma diagramática, as correlações com os campos compostos que aparecem nas identidades estruturais derivadas. 
Para a forma alternativa do funcional gerador de primeira ordem, tomemos a derivada funcional em relação a $J_{\mu \nu}^{b}(y)$ na Eq. (3.27)

$$
\left\langle 0\left|T F^{a \mu \nu}(x) F^{b \rho \sigma}(y)\right| 0\right\rangle_{J}=\frac{1}{i} \frac{\delta}{\delta J_{\rho \sigma}^{b}(y)} \int \mathcal{D} A_{\mu}^{a} \mathcal{D} c^{a} \mathcal{D} \bar{c}^{a}\left[f^{a \mu \nu}(x)-2 J^{a \mu \nu}(x)\right] \exp i S^{(1,2)} .
$$

A derivada atuando na exponencial de $i S^{(1,2)}$ leva nas contribuições:

$$
\left\langle 0\left|T f^{a \mu \nu}(x)\left[f^{b \rho \sigma}(y)-2 J^{b \rho \sigma}(y)\right]\right| 0\right\rangle-2\left\langle 0\left|J^{a \mu \nu}(x)\left[f^{b \rho \sigma}(y)-2 J^{b \rho \sigma}(y)\right]\right| 0\right\rangle_{J},
$$

enquanto atuando no termo dependente da fonte $J$ no integrando do lado direito da Eq. (3.37) resulta em

$$
-\frac{2}{i} \delta^{a b} I^{\mu \nu \rho \sigma} \delta(x-y)\langle 0 \mid 0\rangle_{J}
$$

onde

$$
I^{\mu \nu \rho \sigma} \equiv \frac{\eta^{\mu \rho} \eta^{\nu \sigma}-\eta^{\mu \sigma} \eta^{\nu \rho}}{2}
$$

é a identidade no espaço das matrizes antissimétricas $A$ que satisfazem a propriedade: $A^{\mu \nu \eta \sigma}=$ $-A^{\nu \mu \eta \sigma}=-A^{\mu \nu \sigma \eta}$.

Para chegar na Eq. (3.39) usamos uma extensão de uma relação elementar no estudo de funcionais integrais;

$$
\frac{\delta J^{a \mu \nu}(x)}{\delta J_{\rho \sigma}^{b}(y)}=\delta^{a b} I^{\mu \nu \rho \sigma} \delta(x-y),
$$

que pode ser trivialmente verificada:

$$
\begin{aligned}
J^{a \mu \nu}(x) & =\int d^{d} y \frac{\delta J^{a \mu \nu}(x)}{\delta J_{\rho \sigma}^{b}(y)} J_{\rho \sigma}^{b}(y) \\
& =\int d^{d} y \delta^{a b} I^{\mu \nu \rho \sigma} \delta(x-y) J_{\rho \sigma}^{b}(y) \\
& =I^{\mu \nu \rho \sigma} \int d^{d} y \delta(x-y) J_{\rho \sigma}^{a}(y) \\
& =I^{\mu \nu \rho \sigma} J_{\rho \sigma}^{a}(x) \\
& =\frac{J^{a \mu \nu}(x)-J^{a \nu \mu}(x)}{2}=J^{a \mu \nu}(x),
\end{aligned}
$$

onde usamos a definição da identidade $I_{\mu \nu \alpha \beta}$ dada pela Eq. (3.40) e a antissimetria da fonte $J^{a \mu \nu}$.

Somando todas essas contribuições para o lado direito da Eq. (3.37) obtemos que

$$
\begin{aligned}
\left\langle 0\left|T F^{a \mu \nu}(x) F^{b \rho \sigma}(y)\right| 0\right\rangle_{J} & =\left\langle 0\left|T\left[f^{a \mu \nu}(x)-2 J^{a \mu \nu}(x)\right]\left[f^{b \rho \sigma}(y)-2 J^{b \rho \sigma}(y)\right]\right| 0\right\rangle_{J} \\
& -\frac{2}{i} \delta^{a b} I^{\mu \nu \rho \sigma} \delta(x-y)\langle 0 \mid 0\rangle_{J} .
\end{aligned}
$$

Com $j=J=0$ na Eq. (3.43) deduzimos ${ }^{9}$ a identidade estrutural [15]

$$
\left\langle 0\left|T F^{a \mu \nu}(x) F^{b \rho \sigma}(y)\right| 0\right\rangle=\left\langle 0\left|T f^{a \mu \nu}(x) f^{b \rho \sigma}(y)\right| 0\right\rangle+2 i \delta^{a b} I^{\mu \nu \rho \sigma} \delta(x-y) .
$$

${ }^{9}$ Aqui estamos usando a normalização do funcional gerador $Z: Z[0] \equiv\langle 0 \mid 0\rangle_{J=0}=1$, conforme a Eq. (B.3). 
Essa identidade relaciona o propagador completo $\left\langle 0\left|T F^{a \mu \nu}(x) F^{b \rho \sigma}(y)\right| 0\right\rangle$ do formalismo de primeira ordem com a correlação $\left\langle 0\left|T f^{a \mu \nu}(x) f^{b \rho \sigma}(y)\right| 0\right\rangle$ calculada no formalismo de segunda ordem.

\section{Funções de Green de 3 ou mais-Pontos}

Também é interessante considerar funções de Green com mais campos, principalmente para obter amplitudes.

Derivando a Eq. (3.27) em relação a $j_{\alpha}^{c}(z)$ e $j_{\beta}^{d}(w)$ geramos a função de Green de 4-pontos:

$$
\begin{aligned}
& \left\langle 0\left|T F^{a \mu \nu}(x) F^{b \rho \sigma}(y) A^{c \alpha}(z) A^{d \beta}(w)\right| 0\right\rangle_{J}=\frac{1}{i^{2}} \frac{\delta^{2} Z_{\mathrm{YM}}^{(1)}}{\delta j_{\alpha}^{c}(z) \delta j_{\beta}^{d}(w)} \\
& =\frac{1}{i^{2}} \frac{\delta^{2}}{\delta j_{\alpha}^{c}(z) \delta j_{\beta}^{d}(w)}\left(\int \mathcal{D} A_{\mu}^{a} \mathcal{D} c^{a} \mathcal{D} \bar{c}^{a}\left[f^{a \mu \nu}(x)-2 J^{a \mu \nu}(x)\right]\left[f^{b \rho \sigma}(y)-2 J^{b \rho \sigma}(y)\right] \exp i S^{(1,2)}\right) \\
& +2 i \delta^{a b} I^{\mu \nu \rho \sigma} \delta(x-y) \frac{1}{i^{2}} \frac{\delta^{2} Z_{\mathrm{YM}}^{(1)}}{\delta j_{\alpha}^{c}(z) \delta j_{\beta}^{d}(w)} \\
& =\left\langle 0\left|T\left[f^{a \mu \nu}(x)-2 J^{a \mu \nu}(x)\right]\left[f^{b \rho \sigma}(y)-2 J^{b \rho \sigma}(y)\right] A^{c \alpha}(z) A^{d \beta}(w)\right| 0\right\rangle_{J} \\
& +2 i \delta^{a b} I^{\mu \nu \rho \sigma} \delta(x-y)\left\langle 0\left|T A^{c \alpha}(z) A^{d \beta}(w)\right| 0\right\rangle_{J} .
\end{aligned}
$$

Com $J=j=0$, a Eq. (3.45), se reduz para a igualdade estrutural

$$
\begin{aligned}
& \left\langle 0\left|T F^{a \mu \nu}(x) F^{b \rho \sigma}(y) A^{c \alpha}(z) A^{d \beta}(w)\right| 0\right\rangle \\
& =\left\langle 0\left|T f^{a \mu \nu}(x) f^{b \rho \sigma}(y) A^{c \alpha}(z) A^{d \beta}(w)\right| 0\right\rangle+2 i \delta^{a b} I^{\mu \nu \rho \sigma} \delta(x-y)\left\langle 0\left|T A^{c \alpha}(z) A^{d \beta}(w)\right| 0\right\rangle .
\end{aligned}
$$

Além de mostrar uma relação entre diferentes correlações, a Eq. (3.46) indica como podemos generalizar a identidade (3.44). É fácil de demostrar que para um número $n$ de campos de calibre $A_{\mu}^{a}$ externos, temos que

$$
\begin{aligned}
& \left\langle 0\left|T F^{a \mu \nu}(x) F^{b \rho \sigma}(y) A^{c_{1} \alpha_{1}} \cdots A^{c_{n} \alpha_{n}}\right| 0\right\rangle \\
& =\left\langle 0\left|T f^{a \mu \nu}(x) f^{b \rho \sigma}(y) A^{c_{1} \alpha_{1}} \cdots A^{c_{n} \alpha_{n}}\right| 0\right\rangle+2 i \delta^{a b} I^{\mu \nu \rho \sigma} \delta(x-y)\left\langle 0\left|T A^{c_{1} \alpha_{1}} \cdots A^{c_{n} \alpha_{n}}\right| 0\right\rangle,
\end{aligned}
$$

onde omitimos os argumentos dos campos de calibre com a definição $A^{c_{n} \alpha_{n}} \equiv A^{c_{n} \alpha_{n}}\left(z_{n}\right)$.

Já a generalização da identidade (3.35) é imediata, conforme [15]

$$
\left\langle 0\left|T F^{a \mu \nu}(x) A^{b_{1} \rho_{1}}\left(y_{1}\right) \cdots A^{b_{n} \rho_{n}}\left(y_{n}\right)\right| 0\right\rangle=\left\langle 0\left|T f^{a \mu \nu}(x) A^{b_{1} \rho_{1}}\left(y_{1}\right) \cdots A^{b_{n} \rho_{n}}\left(y_{n}\right)\right| 0\right\rangle
$$

Comparando a identidade (3.43) com as identidades (3.31) e (3.35) notamos que surge um termo proporcional a uma função delta $\delta(x-y)$, ou seja, um termo de contato, tais termos aparecem em diversos contextos como nas bem conhecidas identidades de Ward-Takahashi [29] e na equação de Schwinger-Dyson [14, 30]. Esses termos serão essenciais para a verificação dessas identidades ao nível árvore.

Mais tarde verificaremos explicitamente, no espaço do momento, as identidades (3.35) e (3.44) ao nível de árvore e a Eq. (3.31) no nível de um loop. Assim podemos testar a validade dessas e de outras identidades estruturais que valem em qualquer ordem, para qualquer dimensão $d$ e calibre $\xi$. Para a verificação da identidade (3.31) já será necessário considerar as divergências que surgem devido a efeitos de curta distância pela presença de campos compostos [15] e que são essenciais para a verificação das identidades estruturais. 


\subsection{Equivalência parcial para o formalismo diagonal}

Aqui mostraremos rapidamente a equivalência parcial para o formalismo diagonal. Conforme fizemos anteriormente, partindo do funcional gerador do formalismo de diagonal (sem fontes)

$$
Z_{\mathrm{YM}}^{(1 d)}[0]=\int \mathcal{D} A_{\mu}^{a} \mathcal{D} H_{\mu \nu}^{a} \exp i \int d^{d} x\left(\mathcal{L}_{\mathrm{YM}}^{(1 d)}+\mathcal{L}_{\mathrm{gf}}(A)+\mathcal{L}_{\mathrm{gh}}(A)\right)
$$

podemos fazer o shift no campo auxiliar $H_{\mu \nu}^{a}[16]$

$$
H_{\mu \nu}^{a}=\tilde{H}_{\mu \nu}^{a}+g f^{a b c} A_{\mu}^{b} A_{\nu}^{c} .
$$

Assim como antes o funcional gerador é invariante, pois a medida não se altera com um shift. A única parte que deve ser alterada com o shift é $\mathcal{L}_{\mathrm{YM}}^{(1 d)}$, aplicando o shift (3.50) na Eq. (2.14) obtemos que

$$
\begin{aligned}
\tilde{\mathcal{L}}_{\mathrm{YM}}^{(1 d)} & =\frac{1}{4}\left(\tilde{H}_{\mu \nu}^{a}+g f^{a b c} A_{\mu}^{b} A_{\nu}^{c}\right)^{2}-\frac{1}{2}\left(\tilde{H}_{\mu \nu}^{a}+f_{\mu \nu}^{a}\right) h^{a \mu \nu}-\frac{1}{4}\left(\partial_{\mu} A_{\nu}^{a}-\partial_{\nu} A_{\mu}^{a}\right)^{2} \\
& =\frac{1}{4}\left(\tilde{H}_{\mu \nu}^{a}+h_{\mu \nu}^{a}\right)^{2}-\frac{1}{2}\left(\tilde{H}_{\mu \nu}^{a}+f_{\mu \nu}^{a}\right) h^{a \mu \nu}-\frac{1}{4}\left(f_{\mu \nu}^{a}-h_{\mu \nu}^{a}\right)^{2} \\
& =\frac{1}{4} \tilde{H}_{\mu \nu}^{a} \tilde{H}^{a \mu \nu}-\frac{1}{4} f_{\mu \nu}^{a} f^{a \mu \nu} .
\end{aligned}
$$

Usando o resultado obtido acima, temos que o funcional gerador da Eq. (3.49) após o shift (3.2) é igual a

$$
\begin{aligned}
Z_{\mathrm{YM}}^{(1 d)}[0] & =\int \mathcal{D} \tilde{H}_{\mu \nu}^{a} \exp i \int d^{d} x\left(\frac{1}{4} \tilde{H}_{\mu \nu}^{a} \tilde{H}^{a \mu \nu}\right) \\
& \times \int \mathcal{D} A_{\mu}^{a} \mathcal{D} c^{a} \mathcal{D} \bar{c}^{a} \exp i \int d^{d} x\left(\mathcal{L}_{\mathrm{YM}}^{(2)}+\mathcal{L}_{\mathrm{gf}}(A)+\mathcal{L}_{\mathrm{gh}}(A)\right) .
\end{aligned}
$$

Da mesma forma que no caso do formalismo de primeira ordem, a primeira integral de trajetória na Eq. (3.52) é uma constante que pode ser descartada e a segunda é igual ao funcional gerador do formalismo de segunda ordem sem fontes. Sendo assim,

$$
Z_{\mathrm{YM}}^{(1 d)}[0]=Z_{\mathrm{YM}}^{(2)}[0]
$$

mostrando a equivalência quântica, sem fontes, do formalismo diagonal e o formalismo de segunda ordem.

\subsection{Equivalência quântica para o formalismo diagonal}

Agora consideraremos as fontes, para isso usaremos o funcional gerador do formalismo diagonal com fontes:

$$
Z_{\mathrm{YM}}^{(1 d)}[j, J]=\int \mathcal{D} A_{\mu}^{a} \mathcal{D} H_{\mu \nu}^{a} \mathcal{D} c^{a} \mathcal{D} \bar{c}^{a} \exp i \int d^{d} x\left(\mathcal{L}_{\mathrm{YM}}^{(1 d)}+\mathcal{L}_{\mathrm{gf}}(A)+\mathcal{L}_{\mathrm{gh}}(A)+j^{a \mu} A_{\mu}^{a}+J^{a \mu \nu} H_{\mu \nu}^{a}\right) .
$$

Poderíamos proceder como no caso do formalismo de primeira ordem e considerar dois shifts separados. Primeiramente, em relação a configuração clássica como na Eq. (3.6) e finalmente 
considerando as fontes como no shift (3.10). Porém, usando a condição (3.7) podemos obter esse shift diretamente.

Para obter o shift conforme a condição (3.7) precisamos obter a configuração clássica do campo auxiliar. Com a presença de fontes é necessário considerá-las também no nível clássico por isso usaremos a lagrangiana efetiva com fontes clássicas, tal que,

$$
\mathcal{L}_{\mathrm{eff}}^{(1 d)}=\mathcal{L}_{\mathrm{YM}}^{(1 d)}+\mathcal{L}_{\mathrm{gf}}(A)+\mathcal{L}_{\mathrm{gh}}(A)+j^{a \mu \nu} A_{\mu \nu}^{a}+J^{a \mu \nu} H_{\mu \nu}^{a} .
$$

As equações de Euler-Lagrange do campo auxiliar para a lagrangiana acima resulta em

$$
H_{\mu \nu}^{a} \approx h_{\mu \nu}^{a}-2 J_{\mu \nu}^{a}=S_{\mu \nu}^{a},
$$

tal que o shift adequado para o caso com fontes deve ser

$$
H_{\mu \nu}^{a}=\tilde{H}_{\mu \nu}^{a}+S_{\mu \nu}^{a}=\tilde{H}_{\mu \nu}^{a}+h_{\mu \nu}^{a}-2 J_{\mu \nu}^{a} .
$$

Esse shift é o mesmo considerado em [16, eq. (2.10)] verificando mais uma vez a validade da condição (3.7).

Fazendo tal shift na Eq. (3.54) chegamos no funcional gerador

$$
\begin{aligned}
& Z_{\mathrm{YM}}^{(1 d)}[j, J]=\int \mathcal{D} \tilde{H}_{\mu \nu}^{a} \exp i \int d^{d} x\left(\frac{1}{4} \tilde{H}_{\mu \nu}^{a} \tilde{H}^{a \mu \nu}\right) \\
& \times \int \mathcal{D} A_{\mu}^{a} \mathcal{D} c^{a} \mathcal{D} \bar{c}^{a} \exp i \int d^{d} x\left(\mathcal{L}_{\mathrm{YM}}^{(2)}+\mathcal{L}_{\mathrm{gf}}(A)+\mathcal{L}_{\mathrm{gh}}(A)+j^{a \mu} A_{\mu}^{a}+J^{a \mu \nu} h_{\mu \nu}^{a}-J^{a \mu \nu} J_{\mu \nu}^{a}\right) .
\end{aligned}
$$

A integral no campo auxiliar $\tilde{H}_{\mu \nu}^{a}$ é uma constante que pode ser descartada e obtemos o resultado conforme [16]. Para $J=0$, a lagrangiana efetiva que aparece no lado direito é igual a lagrangiana efetiva do formalismo de segunda ordem.

Reescrevendo a Eq. (3.58) como

$$
Z_{\mathrm{YM}}^{(1 d)}[j, J]=\int \mathcal{D} A_{\mu}^{a} \mathcal{D} c^{a} \mathcal{D} \bar{c}^{a} \exp i \int d^{d} x\left(\mathcal{L}_{\text {eff }}^{(2)}+j^{a \mu} A_{\mu}^{a}+J^{a \mu \nu} h_{\mu \nu}^{a}-J^{a \mu \nu} J_{\mu \nu}^{a}\right) .
$$

é evidente a equivalência entre os funcionais geradores (2.11) e (2.24), isso é, quando $J=0$, temos que [16]

$$
Z_{\mathrm{YM}}^{(1 d)}[j, 0]=Z_{\mathrm{YM}}^{(2)}[j]
$$

Sendo assim, as funções de Green com campos de calibre $A_{\mu}^{a}$ externos no formalismo diagonal são as mesmas que no formalismo de segunda ordem:

$$
\begin{aligned}
\left\langle 0\left|T A^{a_{1} \mu_{1}}\left(x_{1}\right) \cdots A^{a_{n} \mu_{n}}\left(x_{n}\right)\right| 0\right\rangle & \left.\equiv(-i)^{n} \frac{\delta^{n} Z_{\mathrm{YM}}^{(2)}[j]}{\delta j_{\mu_{1}}^{a_{1}}\left(x_{1}\right) \cdots \delta j_{\mu_{n}}^{a_{n}}\left(x_{n}\right)}\right|_{j=0} \\
& =\left.(-i)^{n} \frac{\delta^{n} Z_{\mathrm{YM}}^{(1 d)}[j, J]}{\delta j_{\mu_{1}}^{a_{1}}\left(x_{1}\right) \cdots \delta j_{\mu_{n}}^{a_{n}}\left(x_{n}\right)}\right|_{J=j=0},
\end{aligned}
$$

tal que, o formalismo de primeira e segunda ordem podem ser ditos equivalentes no nível quântico.

Em [9, Apêndice B] mostra-se que a autoenergia do campo de calibre $A_{\mu}^{a}$ no formalismo diagonal e no formalismo de segunda ordem são iguais. Pelas regras de Feynman derivadas no 
apêndice C é evidente que a correção quântica de um loop para o propagador do campo de calibre deve ser a mesma já que o propagador livre do campo $A_{\mu}^{a}$ é igual em ambos formalismos. Isso confirma que a Eq. (3.61) com $n=2$ é válida até um loop.

Para $J \neq 0$, o lado direito da Eq. (3.59) pode ser visto como uma extensão do funcional gerador do formalismo de segunda ordem dado na Eq. (2.11). Pois, além de gerar funções de Green para o campo de calibre $A_{\mu}^{a}$ também gera funções de Green para o operador composto

$$
h_{\mu \nu}^{a}(x)=g f^{a b c} A_{\mu}^{b}(x) A_{\nu}^{c}(x) .
$$

Além disso, a dependência quadrática em $J$ na Eq. (3.59) tem a mesma origem da encontrada na Eq. (3.13).

Na próxima seção consideraremos em mais detalhes o funcional gerador obtido na Eq. (3.59), onde mostraremos que as funções de Green com o campo auxiliar $H_{\mu \nu}^{a}$ externo estão relacionadas com funções de Green com o campo composto $h_{\mu \nu}^{a}(x)$.

\subsubsection{Identidades estruturais}

Nessa seção obteremos identidades estruturais análogas as obtidas no caso do formalismo de primeira ordem. Para isso poderíamos diretamente considerar as duas formas para o funcional gerador do formalismo diagonal dado pelas Eqs. (3.8) e (3.59). Contudo, note que pela Eq. (3.26) e a expressão da forma alternativa do funcional gerador do formalismo de primeira ordem (3.24) que pode-se obter os termos — análogos — no formalismo diagonal com as substituições:

$$
\begin{aligned}
Z_{\mathrm{YM}}^{(1)} & \rightarrow Z_{\mathrm{YM}}^{(1 d)}, \\
F & \rightarrow H, \\
f & \rightarrow h .
\end{aligned}
$$

As duas últimas são suficientes para obtemos as identidades estruturais análogas às já derivadas para o formalismo de primeira ordem na seção 3.3.

Usando as substituições acima nas Eqs. (3.32), (3.35) e (3.36) chegamos nas seguintes identidades estruturais para o formalismo diagonal:

$$
\begin{gathered}
\left\langle 0\left|T H_{\mu \nu}^{a}(x)\right| 0\right\rangle=\left\langle 0\left|T h_{\mu \nu}^{a}(x)\right| 0\right\rangle, \\
\left\langle 0\left|T H_{\mu \nu}^{a}(x) A_{\rho}^{b}(y)\right| 0\right\rangle=\left\langle 0\left|T h_{\mu \nu}^{a}(x) A_{\rho}^{b}(y)\right| 0\right\rangle, \\
\left\langle 0\left|T H_{\mu \nu}^{a}(x) H_{\rho \sigma}^{b}(y)\right| 0\right\rangle=2 i \delta(x-y) \delta^{a b} I_{\mu \nu \rho \sigma}+\left\langle 0\left|T h_{\mu \nu}^{a}(x) h_{\rho \sigma}^{b}(y)\right| 0\right\rangle .
\end{gathered}
$$

As demais identidades estruturais (como as generalizações das identidade acimas) também podem ser obtidas trivialmente usando as substituições (3.63). Ou diretamente através da comparação das duas formas do funcional gerador $Z_{\mathrm{YM}}^{(1 d)}$ conforme feito em detalhes para o formalismo de primeira ordem.

A validade de tais identidades será verificada explicitamente, no espaço do momento, no Cap. 6 , onde verificaremos perturbativamente as identidades (3.64b) e (3.64c) em até um loop. A 
escolha dessas identidades, obtidas no formalismo diagonal, em vez das obtidas no formalismo de primeira ordem possui duas motivações.

Primeiramente, do campo composto $f_{\mu \nu}^{a}(x)$ só temos um termo que é propriamente composto que é justamente $h_{\mu \nu}^{a}$. Sendo assim, as contribuições que devem surgir devido a presença dos campos compostos são dadas exclusivamente por correlações com o campo $h_{\mu \nu}^{a}$. Logo, pela definição do tensor intensidade de campo (2.5), as contribuições do formalismo de primeira ordem são combinações lineares de resultados obtidos no formalismo diagonal.

Por exemplo, para a identidade (3.35):

$$
\left\langle 0\left|T f_{\mu \nu}^{a}(x) A_{\rho}^{b}(y)\right| 0\right\rangle=\left\langle 0\left|T\left[\partial_{\mu} A_{\nu}^{a}(x)-\partial_{\nu} A_{\mu}^{a}(x)\right] A_{\rho}^{b}(y)\right| 0\right\rangle+\left\langle 0\left|T h_{\mu \nu}^{a}(x) A_{\rho}^{b}(y)\right| 0\right\rangle .
$$

O primeiro termo é apenas uma combinação linear de propagadores inteiros do campo de calibre e o segundo pode ser obtido através da identidade (3.64b). As divergências devido ao primeiro termo são divergências usuais do tipo UV, enquanto as divergência do segundo termo são mais interessantes já que surgem devido a presença de campos compostos. Enquanto existe diversos métodos para computação (sendo o resultado amplamente conhecido) da correção a nível de um loop do primeiro termo, a computação das divergências devido aos campo compostos é mais obscura na literatura.

Além disso, no formalismo diagonal não temos o acoplamento dos campos auxiliar $H_{\mu \nu}^{a}$ e o campo de calibre $A_{\mu}^{a}$ evidenciando as contribuições do campo composto $h_{\mu \nu}^{a}(x)$. Esse acoplamento é responsável por termos como o segundo termo da Eq. (3.65) que são simplesmente combinações lineares de funções de Green computadas no formalismo de segunda ordem.

A segunda razão é pautada no fato que para gravitação a dificuldade para a verificação das identidades estruturais é muito maior. Podemos citar dois fatores relevantes para essa diferença: o propagador do campo do gráviton não é transverso e o aumento na ordem dos campos tensoriais considerados que levam a bases tensoriais bem mais complexas. Apesar de que as formulações de primeira ordem (e diagonal) da teoria de YM e da gravitação sejam diferentes, elas são análogas. No caso da gravitação, o formalismo diagonal é a melhor alternativa para a verificação das identidades estruturais, embora ainda seja complicado. Por isso considerar anteriormente na teoria de YM uma formulação análoga nos ajudará a entender os resultados análogos (porém, diferentes) obtidos na gravitação. Ressaltamos que dentro dessas semelhanças entre a teoria de YM e a gravitação está incluída a condição (3.7) que pode ser trivialmente estendida para a gravitação.

\subsection{Equivalência geral}

Considerando as Eqs. (3.14) e (3.60), temos que

$$
Z_{\mathrm{YM}}^{(2)}[j]=Z_{\mathrm{YM}}^{(1)}[j, 0]=Z_{\mathrm{YM}}^{(1 d)}[j, 0]
$$

mostrando que todos os formalismos considerados para a teoria de YM são equivalentes no nível quântico; as funções de Green com campos de calibre $A_{\mu}^{a}$ são iguais em todos os formalismos:

$$
\left.\frac{\delta^{n} Z_{\mathrm{YM}}^{(2)}[j]}{\delta j_{\mu_{1}}^{a_{1}}\left(x_{1}\right) \cdots \delta j_{\mu_{n}}^{a_{n}\left(x_{n}\right)}}\right|_{j=0}=\left.\frac{\delta^{n} Z_{\mathrm{YM}}^{(1)}[j, J]}{\delta j_{\mu_{1}}^{a_{1}}\left(x_{1}\right) \cdots \delta j_{\mu_{n}}^{a_{n}}\left(x_{n}\right)}\right|_{J=j=0}=\left.\frac{\delta^{n} Z_{\mathrm{YM}}^{(1 d)}[j, J]}{\delta j_{\mu_{1}}^{a_{1}}\left(x_{1}\right) \cdots \delta j_{\mu_{n}}^{a_{n}}\left(x_{n}\right)}\right|_{J=j=0} .
$$


Além disso, podemos obter relações entre os formalismos de primeira ordem e diagonal que mostram relações interessantes entre funções de Green e as correlações das suas configurações clássicas. Considere as Eqs. (3.36) e (3.44) podemos eliminar os termos de contato obtendo:

$$
\left\langle 0\left|T F_{\mu \nu}^{a}(x) F_{\alpha \beta}^{b}(y)\right| 0\right\rangle-\left\langle 0\left|T H_{\mu \nu}^{a}(x) H_{\alpha \beta}^{b}(y)\right| 0\right\rangle=\left\langle 0\left|T f_{\mu \nu}^{a}(x) f_{\alpha \beta}^{b}(y)\right| 0\right\rangle-\left\langle 0\left|T h_{\mu \nu}^{a}(x) h_{\alpha \beta}^{b}(y)\right| 0\right\rangle .
$$

A relação acima mostra que a diferença entre os dois propagadores inteiros é dada pela diferença entre as correlações de 2-pontos das suas configurações clássicas. Outra relação similar pode ser obtida das Eqs. (3.35) e (3.64b):

$$
\left\langle 0\left|T F_{\mu \nu}^{a}(x) A_{\rho}^{b}(y)\right| 0\right\rangle-\left\langle 0\left|T H_{\mu \nu}^{a}(x) A_{\rho}^{b}(y)\right| 0\right\rangle=\left\langle 0\left|T f_{\mu \nu}^{a}(x) A_{\rho}^{b}(y)\right| 0\right\rangle-\left\langle 0\left|T h_{\mu \nu}^{a}(x) A_{\rho}^{b}(y)\right| 0\right\rangle .
$$

As relações derivadas acima são equivalentes as identidades estruturais obtidas pela comparação dos funcionais geradores do formalismo de primeira ordem e do formalismo diagonal. Assim obtemos um conjunto de identidades que são consequência da equivalência entre todas as formulações consideradas. Embora sejam equivalentes, tais relações não são iguais. Essas relações são mais restritas já que elas decorrem da equivalência geral entre todos os formalismos, conforme a Eq. (3.67).

Podemos entender a Eq. (3.66) para $J \neq 0$ :

$$
Z_{\mathrm{YM}}^{(1)}[j, J]=\tilde{Z}_{\mathrm{YM}}^{(1 d)}[j, J]=\tilde{Z}_{\mathrm{YM}}^{(2)}[j, J]
$$

onde

$$
\begin{aligned}
\tilde{Z}_{\mathrm{YM}}^{(1 d)}[j, K, J] & =\int \mathcal{D} H_{\mu \nu}^{a} \mathcal{D} A_{\mu}^{a} \mathcal{D} c^{a} \mathcal{D} \bar{c}^{a} \exp i \int d^{d} x\left[\mathcal{L}_{\mathrm{YM}}^{(1 d)}+\mathcal{L}_{\mathrm{gf}}(A)+\mathcal{L}_{\mathrm{gh}}(A)+j^{a \mu} A_{\mu}^{a}\right. \\
& \left.+J^{a \mu \nu}\left(H_{\mu \nu}^{a}+f_{\mu \nu}^{a}-h_{\mu \nu}^{a}\right)\right]
\end{aligned}
$$

$\mathrm{e}$

$$
\begin{aligned}
\tilde{Z}_{\mathrm{YM}}^{(2)}[j, J] & =\int \mathcal{D} A_{\mu}^{a} \mathcal{D} c^{a} \mathcal{D} \bar{c}^{a} \exp i \int d^{d} x\left(\mathcal{L}_{\mathrm{YM}}^{(2)}+\mathcal{L}_{\mathrm{gf}}(A)+\mathcal{L}_{\mathrm{gh}}(A)+j^{a \mu} A_{\mu}^{a}\right. \\
& \left.+J^{a \mu \nu} f_{\mu \nu}^{a}+J_{\mu \nu}^{a} J^{a \mu \nu}\right) .
\end{aligned}
$$

Quando temos $J=0$ é claro que a Eq. (3.70) se reduz a Eq. (3.66).

Os funcionais geradores acima podem ser visto como generalizações para os funcionais geradores $Z_{\mathrm{YM}}^{(1 d)}[j, 0]$ e $Z_{\mathrm{YM}}^{(2)}[j]$ derivadas do funcional gerador do formalismo de primeira ordem $Z_{\mathrm{YM}}^{(1)}$. Note que o funcional gerador do formalismo de primeira ordem na forma dada na Eq. (3.13) é igual ao funcional gerador generalizado para o formalismo de segunda ordem definido na Eq. (3.72). Já o funcional gerador generalizado do formalismo diagonal é obtido através do shift $F_{\mu \nu}^{a}=H_{\mu \nu}^{a}+f_{\mu \nu}^{a}-h_{\mu \nu}^{a}$ no funcional gerador $Z_{\mathrm{YM}}^{(1)}[j, J]$.

As correlações geradas pela fonte $J$ para esses funcionais generalizados são iguais as funções de Green no formalismo de primeira ordem. Essa igualdade dá a origem à identidades estruturais como as identidades (3.68), (3.69), (3.35) e (3.44). Esse procedimento pode ser adotado para outros formalismos. Um exemplo é o funcional gerador obtido na Eq. (3.59) que também pode ser visto como um funcional gerador generalizado para o formalismo de segunda ordem, porém nesse caso é derivado do funcional gerador do formalismo diagonal. 
A abordagem acima permite que vejamos a equivalência de uma forma mais simétrica e que todos os formalismos são equivalentes de forma geral. Onde não só consideramos os graus de liberdades físicos do campo $A_{\mu}^{a}$, mas também do campo auxiliar $F_{\mu \nu}^{a}$ e $H_{\mu \nu}^{a}$. Tal que, de forma genérica, podemos afirmar que

$$
\begin{aligned}
\text { Formalismo de Segunda Ordem } & \equiv \text { Formalismo de Primeira Ordem } \\
& \equiv \text { Formalismo Diagonal. }
\end{aligned}
$$

No próximo capítulo essa análise vai ser estendida para a gravitação, conforme [16]. Como na teoria de YM, também estudaremos três formulações para gravitação, esses formalismos são análogos aos estudados para a teoria de YM. Embora sejam análogos, mostraremos que têm diferentes intrínsecas, além de serem mais complexos devido a complexidade da gravitação em si. 


\section{CAPÍTULO 4}

\section{GravitaÇÃo}

Neste capítulo mostraremos a quantização da gravitação em três formulações análogas às estudadas na teoria de YM. Para a quantização da gravitação é necessário considerar uma aproximação de campo fraco onde usamos a métrica de Minkowski como campo de fundo.

Assim como no caso da teoria de YM iremos apenas considerar a teoria pura, ou seja, sem campos de matéria, além disso também não vamos considerar a constante cosmológica $(\Lambda=0)$.

\subsection{Ação de Einstein-Hilbert}

A ação da formulação de segunda ordem da gravitação é a ação de Einstein-Hilbert [16]

$$
S_{\mathrm{EH}}=-\frac{1}{16 \pi G_{N}} \int d^{d} x \sqrt{-g} g^{\mu \nu} R_{\mu \nu}(\Gamma),
$$

onde $G_{N}$ é a constante da gravitação de Newton e $R_{\mu \nu}(\Gamma)$ é o tensor de Ricci.

O tensor de Ricci pode ser dado em termos da conexão afim por ${ }^{1}$

$$
R_{\mu \nu}(\Gamma)=\Gamma_{\mu \rho, \nu}^{\rho}-\Gamma_{\mu \nu, \rho}^{\rho}-\Gamma_{\mu \nu}^{\sigma} \Gamma_{\sigma \rho}^{\rho}+\Gamma_{\mu \sigma}^{\rho} \Gamma_{\nu \rho}^{\sigma},
$$

onde as componentes $\Gamma_{\mu \nu}^{\lambda}$ da conexão afim de Levi-Civita ${ }^{2}$ são os símbolos de Christoffel dados em termos da métrica por

$$
\Gamma_{\mu \nu}^{\lambda}=\frac{1}{2} g^{\lambda \sigma}\left(g_{\mu \sigma, \nu}+g_{\nu \sigma, \mu}-g_{\mu \nu, \sigma}\right) .
$$

A variação da ação de Einstein-Hilbert pela métrica $g^{\mu \nu}$ leva às conhecidas equações de campo de Einstein [3]

$$
R_{\mu \nu}-\frac{1}{2} g_{\mu \nu} R=8 \pi G_{N} T_{\mu \nu},
$$

\footnotetext{
${ }^{1}$ Usamos a notação $A_{, \mu} \equiv \partial_{\mu} A$.

${ }^{2}$ Uma conexão de Levi-Civita é uma conexão que preserva a métrica (metricidade [31, pág. 117]) possui torção nula [31, eq. 2.7]. Conexões com torção ou que não presevam a métrica não são consideradas nesse trabalho.
} 
onde $R=R_{\mu \nu} g^{\mu \nu}$ e $T_{\mu \nu}$ é o tensor de energia-momento.

Em vez da métrica $g^{\mu \nu}$ usaremos

$$
h^{\mu \nu} \equiv \sqrt{-g} g^{\mu \nu}
$$

que permite reescrevemos a ação (4.1) como

$$
S_{\mathrm{EH}}=\int d^{d} x \mathcal{L}_{\mathrm{EH}}^{\mathrm{II}}
$$

onde, conforme [32],

$$
\mathcal{L}_{\mathrm{EH}}^{\mathrm{II}}=-\frac{1}{4 \kappa^{2}}\left(h^{\rho \sigma} h_{\lambda \alpha} h_{\kappa \tau} h^{\alpha \kappa}{ }_{, \rho} h^{\lambda \tau}{ }_{, \sigma}-\frac{1}{d-2} h^{\rho \sigma} h_{\alpha \kappa} h_{\lambda \tau} h^{\alpha \kappa}{ }_{, \rho} h^{\lambda \tau}{ }_{, \sigma}-2 h_{\alpha \tau} h^{\alpha \kappa}{ }_{, \rho} h^{\rho \tau}{ }_{, \kappa}\right),
$$

com $\kappa=\sqrt{16 \pi G_{N}}$. A lagrangiana acima tem uma dependência na dimensão $d$, sendo que é mal definida para $d=2$, por isso consideraremos $d>2$.

A gravitação possui uma simetria local pela sua invariância por transformações locais de coordenadas. Uma transformação de coordenadas pode ser expressa de forma infinitesimal como

$$
\delta x^{\mu}=\theta^{\mu}(x)
$$

Tal transformação de coordenadas induz a uma transformação da métrica e sua determinante, e portanto de $h^{\mu \nu}$, já que são densidade tensoriais. Assim a transformação de coordenadas (4.8) induz na variação [33]

$$
\delta h^{\mu \nu}=h^{\mu \rho} \partial_{\rho} \theta^{\nu}+h^{\nu \rho} \partial_{\rho} \theta^{\mu}-\partial_{\rho}\left(h^{\mu \nu} \theta^{\rho}\right) .
$$

Essas transformações de coordenadas podem ser interpretadas como transformações de calibre, tal que, a gravitação pode ser vista como uma teoria de calibre não abeliana (o grupo de calibre da gravitação seria um grupo não compacto: o grupo de Poincaré). Isso permite que possamos tentar quantizar a gravitação como uma teoria quântica de campo via integral de trajetória usando o procedimento de Faddeev-Popov.

\subsubsection{Quantização}

Como já vimos as transformações locais da forma da Eq. (4.8) podem ser vistas como transformações de calibre não abelianas. Podemos então tentar quantizar a gravitação como uma teoria de calibre usando $h^{\mu \nu}$ como o campo de calibre, porém isso não é possível ${ }^{3}$ [5]. Precisamos considerar a aproximação de campo fraco com um campo de fundo ${ }^{4}$, no caso usaremos a métrica de Minkowski, tal que

$$
h^{\mu \nu}(x)=\eta^{\mu \nu}+\kappa \phi^{\mu \nu}(x),
$$

onde o campo $\phi^{\mu \nu}$ toma o papel do campo de calibre da gravitação cuja partícula é denominada "gráviton". Além disso, com a definição (4.10) passamos a usar a métrica plana $\eta^{\mu \nu}$ para subir e baixar os índices [5].

\footnotetext{
${ }^{3}$ Pela Eq. (4.7) não temos um termo bilinear em $h^{\mu \nu}$, ou seja, não podemos definir um propagador.

${ }^{4}$ Conforme [34], podemos escolher qualquer métrica contínua como campo de fundo.
} 
Antes de continuar é necessário usar o procedimento de Faddeev-Popov para remover os graus de liberdades não físicos devido a presença da invariância de calibre na ação de EH. Os detalhes do procedimento para a gravitação é encontrado na Ref. [32, Apêndice B].

Primeiramente precisamos fixar um calibre. Podemos então adicionar um termo de fixação de calibre como [32]

$$
\mathcal{L}_{\mathrm{gf}}(\phi)=-\frac{1}{2 \alpha \kappa^{2}}\left(\partial_{\mu} h^{\mu \nu}\right)^{2}=-\frac{1}{2 \alpha}\left(\partial_{\mu} \phi^{\mu \nu}\right)^{2},
$$

onde usamos que $\partial_{\mu} h^{\mu \nu}=\partial_{\mu}\left(\eta^{\mu \nu}+\kappa \phi^{\mu \nu}\right)=\kappa \partial_{\mu} \phi^{\mu \nu}$. Esse termo de fixação de calibre é análogo ao termo (2.8) usado na quantização da teoria de YM.

O calibre generalizado com $\alpha=1$ é conhecido como calibre de DeDonder. De forma semelhante que o calibre de Feynman leva a uma simplificação na estrutura do propagador do campo de calibre. Essa é uma das razões para que o calibre $\alpha=1$ também seja conhecido como calibre de DeDonder-Feynman.

Com a condição de calibre $\partial_{\mu} \phi^{\mu \nu}=0$ sendo fixada pelo termo (4.11) implica num termo devido aos campos fantasmas $d_{\nu}, \bar{d}_{\mu}$ igual a [5]

$$
\mathcal{L}_{\mathrm{gh}}(\phi)=\bar{d}_{\mu}\left\{\partial^{2} \eta^{\mu \nu}+\kappa\left[\left(\phi_{, \rho}^{\rho \sigma}\right) \partial_{\sigma} \eta^{\mu \nu}-\left(\phi_{, \rho}^{\rho \mu}\right) \partial^{\nu}+\phi^{\rho \sigma} \partial_{\rho} \partial_{\sigma} \eta^{\mu \nu}-\left(\partial_{\rho} \partial^{\nu} \phi^{\rho \mu}\right)\right]\right\} d_{\nu}
$$

Logo, a lagrangiana efetiva para gravitação no formalismo de segunda ordem é dada por

$$
\mathcal{L}_{\text {eff }}^{\text {II }}=\mathcal{L}_{\text {EH }}^{\mathrm{II}}+\mathcal{L}_{\text {gf }}(\phi)+\mathcal{L}_{\text {gh }}(\phi)
$$

A quantização segue pelo funcional gerador $Z$ :

$$
Z_{\mathrm{EH}}^{\mathrm{II}}[j]=\int \mathcal{D} d_{\nu} \mathcal{D} \bar{d}_{\mu} \mathcal{D} \phi^{\mu \nu} \exp i\left[S_{\mathrm{EH}}+\int d^{d} x\left(\mathcal{L}_{\mathrm{gf}}(\phi)+\mathcal{L}_{\mathrm{gh}}(\phi)+j_{\mu \nu} \phi^{\mu \nu}\right)\right],
$$

onde a ação $S_{\mathrm{EH}}$ é dada na Eq. (4.6) fazendo $h^{\mu \nu}=\eta^{\mu \nu}+\kappa \phi^{\mu \nu}$.

Porém, enquanto $h^{\mu \nu} \equiv \sqrt{-g} g^{\mu \nu}=\eta^{\mu \nu}+\kappa \phi^{\mu \nu}$, o campo $h_{\mu \nu}=g_{\mu \nu} / \sqrt{-g}$ é dado por uma série de potência (infinita) em $\kappa$ :

$$
h_{\mu \nu}=\eta_{\mu \nu}-\kappa \phi_{\mu \nu}+\kappa^{2} \phi_{\mu \rho} \phi_{\nu \rho}+\mathcal{O}\left(\kappa^{3}\right)
$$

Portanto, usando as Eqs. (4.10) e (4.15) na lagrangiana (4.7) obtemos infinitos termos de interação, tal que, a lagrangiana efetiva pode ser então escrita como

$$
\mathcal{L}_{\text {eff }}^{\mathrm{II}}=\mathcal{L}_{\text {bilinear }}^{\mathrm{II}}+\sum_{n=3}^{\infty} \kappa^{i-2} V^{(n)},
$$

onde $\mathcal{L}_{\text {bilinear }}^{\mathrm{II}}$ é a parte bilinear no campo $\phi^{\mu \nu}$ e $V^{(n)}$ são termos de interação de $n$ campos. Vemos então que a formulação de segunda ordem da gravitação leva a um número infinito de vértices.

Algumas das regras de Feynman para gravitação serão dadas mais tarde no formalismo diagonal. Como no caso da formulação diagonal da teoria de YM, temos que o vértice cúbico $(\phi \phi \phi)$ e propagador do campo do gráviton são os mesmos da formulação de segunda ordem [6]. Além disso, o setor do campo de fantasma também não é alterado. 


\subsection{Formalismo de primeira ordem}

Assim como na teoria de YM, a gravitação pode ser formulada num formalismo de primeira ordem. Esse formalismo é (conforme [18], erroneamente ${ }^{5}$ ) conhecido na literatura como Formalismo de Palatini. O formalismo de primeira ordem é derivado da ação de Einstein-Hilbert definida na Eq. (4.1), onde consideramos a métrica $g^{\mu \nu}$ e a conexão afim $\Gamma$ como campos independentes.

Assumindo que a métrica e a conexão afim são simétricas pode-se mostrar que a equação de movimento para a conexão afim resulta na Eq. (4.3), ou seja, as equações de movimento para a conexão afim $\Gamma$ implicam numa conexão de Levi-Civita. Isso mostra que o formalismo de primeira ordem é classicamente equivalente ${ }^{6}$ ao formalismo de segunda ordem.

Porém, invés de considerar $g^{\mu \nu}$ e $\Gamma_{\mu \nu}^{\lambda}$ de forma independente ${ }^{7}$ é mais útil [5] considerar $h^{\mu \nu}$, já definido na Eq. (4.5), e

$$
G_{\mu \lambda}^{\lambda}=\Gamma_{\mu \nu}^{\lambda}-\frac{1}{2}\left(\delta_{\mu}^{\lambda} \Gamma_{\nu \alpha}^{\alpha}+\delta_{\nu}^{\lambda} \Gamma_{\mu \alpha}^{\alpha}\right)
$$

de forma independente. Assim, a lagrangiana de Einstein-Hilbert (como definida na Eq. (4.6)) é dada na formulação de primeira ordem por

$$
\mathcal{L}_{\mathrm{EH}}^{\mathrm{I}}=\frac{h^{\mu \nu}}{\kappa^{2}}\left(G_{\mu \nu, \lambda}^{\lambda}+\frac{1}{d-1} G_{\mu \lambda}^{\lambda} G_{\nu \sigma}^{\sigma}-G_{\mu \sigma}^{\lambda} G_{\nu \lambda}^{\sigma}\right)
$$

onde o campo $G_{\mu \nu}^{\lambda}$ tem um papel auxiliar similar ao campo $F_{\mu \nu}^{a}$ do formalismo de primeira ordem da teoria de YM.

Podemos facilmente demonstrar que a lagrangiana do formalismo de primeira ordem dada pela Eq. (4.18) é equivalente (no nível clássico) a lagrangiana definida pela ação dada na Eq. (4.1). Consideremos primeiramente o termo com a derivada $G_{\mu \nu, \lambda}^{\lambda}$; derivando a Eq. (4.17) obtemos

$$
G_{\mu \nu, \lambda}^{\lambda}=\Gamma_{\mu \nu, \lambda}^{\lambda}-\frac{1}{2}\left(\Gamma_{\nu \sigma, \nu}^{\sigma}+\Gamma_{\mu \sigma, \nu}^{\sigma}\right)=\Gamma_{\mu \nu, \lambda}^{\lambda}-\Gamma_{\mu \sigma, \nu}^{\sigma},
$$

onde usamos que $\Gamma_{\mu \sigma, \nu}^{\sigma}=\Gamma_{\nu \sigma, \mu}^{\sigma} \cdot{ }^{8}$ Como esperado a contribuição acima é igual ao oposto dos termos (com derivada) do tensor de Ricci definido na Eq. (4.2).

\footnotetext{
${ }^{5}$ Segundo [18], fora Einstein que obteve os primeiros resultados sobre princípios variacionais da ação da relatividade geral que consideravam variações da métrica e da conexão afim de forma independente.

${ }^{6}$ No caso da gravitação pura, sem campos de matéria.

${ }^{7}$ Note que estamos considerando que a conexão é simétrica (sem torção), isso é, $\Gamma_{\mu \nu}^{\lambda}=\Gamma_{\nu \mu}^{\lambda}$. No artigo [35] demostra-se a equivalência entre os formalismos de primeira e segunda ordem considerando uma conexão arbitrária mostrando que pela invariância de calibre temos $\tilde{\Gamma}_{\mu \nu}^{\lambda} \rightarrow \Gamma_{\mu \nu}^{\lambda}+\delta_{\nu}^{\lambda} U_{\mu}$, onde $\Gamma_{\mu \nu}^{\lambda}$ é a conexão de Levi-Civita. Sendo assim, considerar o caso de uma conexão sem torção é equivalente a considerar o caso geral sem os graus de liberdade não físicos devido a invariância de calibre por transformações projetivas [35]. Conforme [36] não há inconsistências em considerar uma conexão sem torção a menos que seja acoplado campos de matéria, como férmions, ao campo gravitacional. Tais inconsistências se devem ao fato que campos de matéria implicam em uma conexão com torção [4, 13] no formalismo de primeira ordem, sendo que a conexão afim no formalismo usual pode ser sempre escolhida sem torção, como a conexão (4.3).

${ }^{8}$ É fácil de mostrar usando a definição dos símbolos de Christoffel dada na Eq. (4.3). Temos que $\Gamma_{\mu \sigma, \nu}^{\sigma}-\Gamma_{\nu \sigma, \mu}^{\sigma}=g^{\sigma \alpha}\left(g_{\mu \alpha, \sigma \nu}-g_{\nu \sigma, \alpha \mu}-\sigma \leftrightarrow \alpha\right)=0$, pois temos a contração de um tensor simétrico (a métrica) e um tensor antissimétrico.
} 
Calculando o termo $G_{\mu \sigma}^{\lambda} G_{\nu \lambda}^{\sigma}$ usando a definição dada na Eq. (4.17) obtemos

$$
\begin{aligned}
\Gamma_{\mu \sigma}^{\lambda} \Gamma_{\nu \lambda}^{\sigma} & =\left[\Gamma_{\mu \sigma}^{\lambda}-\frac{1}{2}\left(\delta_{\mu}^{\lambda} \Gamma_{\sigma \alpha}^{\alpha}+\delta_{\sigma}^{\lambda} \Gamma_{\mu \alpha}^{\alpha}\right)\right]\left[\Gamma_{\nu \lambda}^{\sigma}-\frac{1}{2}\left(\delta_{\nu}^{\sigma} \Gamma_{\lambda \beta}^{\beta}+\delta_{\lambda}^{\sigma} \Gamma_{\nu \beta}^{\beta}\right)\right] \\
& =\Gamma_{\mu \sigma}^{\lambda} \Gamma_{\nu \lambda}^{\sigma}-\Gamma_{\mu \nu}^{\lambda} \Gamma_{\lambda \alpha}^{\alpha}-\Gamma_{\mu \lambda}^{\lambda} \Gamma_{\nu \beta}^{\beta}+\frac{1}{4}(d+3) \Gamma_{\mu \alpha}^{\alpha} \Gamma_{\nu \beta}^{\beta} \\
& =\Gamma_{\mu \sigma}^{\lambda} \Gamma_{\nu \lambda}^{\sigma}-\Gamma_{\mu \nu}^{\lambda} \Gamma_{\lambda \alpha}^{\alpha}+\frac{1}{4}(d-1) \Gamma_{\mu \alpha}^{\alpha} \Gamma_{\nu \beta}^{\beta},
\end{aligned}
$$

onde simplificamos os termos mistos (termos com um delta de Kronecker) usando a simetria dos índices: $\Gamma_{\mu \nu}^{\lambda}=\Gamma_{\nu \mu}^{\lambda}$. Finalmente, podemos computar o último termo $G_{\mu \lambda}^{\lambda} G_{\nu \sigma}^{\sigma}$; contraindo os índices da Eq. (4.17):

$$
\begin{aligned}
G_{\mu \lambda}^{\lambda} & =\Gamma_{\mu \lambda}^{\lambda}-\frac{1}{2}\left(\delta_{\mu}^{\lambda} \Gamma_{\lambda \alpha}^{\alpha}+\delta_{\lambda}^{\lambda} \Gamma_{\mu \alpha}^{\alpha}\right) \\
& =\Gamma_{\mu \lambda}^{\lambda}\left(1-\frac{1+d}{2}\right)=\Gamma_{\mu \lambda}^{\lambda} \frac{1-d}{2}
\end{aligned}
$$

$\log 0$

$$
G_{\mu \lambda}^{\lambda} G_{\nu \sigma}^{\sigma}=\Gamma_{\mu \lambda}^{\lambda} \Gamma_{\nu \sigma}^{\sigma} \frac{(1-d)^{2}}{4}
$$

Substituindo os resultados obtidos nas Eqs. (4.19), (4.20) e (4.22) na Eq. (4.18) obtemos que

$$
\begin{aligned}
\mathcal{L}_{\mathrm{EH}}^{\mathrm{I}} & =\frac{h^{\mu \nu}}{\kappa^{2}}\left(G_{\mu \nu, \lambda}^{\lambda}+\frac{1}{d-1} G_{\mu \lambda}^{\lambda} G_{\nu \sigma}^{\sigma}-G_{\mu \sigma}^{\lambda} G_{\nu \lambda}^{\sigma}\right) \\
& =\frac{h^{\mu \nu}}{\kappa^{2}}\left[\Gamma_{\mu \nu, \lambda}^{\lambda}-\Gamma_{\mu \sigma, \nu}^{\sigma}+\frac{d-1}{4} \Gamma_{\mu \lambda}^{\lambda} \Gamma_{\nu \sigma}^{\sigma}-\left(\Gamma_{\mu \sigma}^{\lambda} \Gamma_{\nu \lambda}^{\sigma}-\Gamma_{\mu \nu}^{\lambda} \Gamma_{\lambda \alpha}^{\alpha}+\frac{1}{4}(d-1) \Gamma_{\mu \alpha}^{\alpha} \Gamma_{\nu \beta}^{\beta}\right)\right] \\
& =-\frac{h^{\mu \nu}}{\kappa^{2}} R_{\mu \nu}=-\frac{1}{\kappa^{2}} \sqrt{-g} g^{\mu \nu} R_{\mu \nu} .
\end{aligned}
$$

Portanto, a lagrangiana do formalismo de primeira ordem é classicamente equivalente a lagrangiana do formalismo de segunda ordem definida pela ação de Einstein-Hilbert na Eq. (4.1). Porém, note que consideramos que a conexão afim era igual a conexão de Levi-Civita. Abaixo mostraremos que podemos estender esse resultado sem considerar isso previamente, mas sim usando que a conexão de Levi-Civita é igual a configuração clássica de $\Gamma_{\mu \nu}^{\lambda}$ (no formalismo de primeira ordem).

Podemos reescrever a Eq. (4.17) de uma forma mais conveniente fatorando o campo auxiliar $G_{\mu \nu}^{\lambda}:$

$$
\tilde{\mathcal{L}}_{\mathrm{EH}}^{\mathrm{I}}=\frac{1}{2 \kappa^{2}} G_{\mu \nu}^{\lambda} M_{\lambda}^{\mu \nu \pi \tau}(h) G_{\pi \tau}^{\rho}-\frac{1}{\kappa^{2}} G_{\mu \nu}^{\lambda} h_{, \lambda}^{\mu \nu},
$$

onde $[6]$

$$
\begin{aligned}
M_{\lambda}^{\mu \nu \pi \tau}(h) & =\frac{1}{2}\left[\frac{1}{d-1}\left(\delta_{\lambda}^{\nu} \delta_{\sigma}^{\tau} h^{\mu \pi}+\delta_{\lambda}^{\mu} \delta_{\sigma}^{\tau} h^{\nu \pi}+\delta_{\lambda}^{\nu} \delta_{\sigma}^{\pi} h^{\mu \tau}+\delta_{\lambda}^{\mu} \delta_{\sigma}^{\pi} h^{\nu \tau}\right)\right. \\
& \left.-\left(\delta_{\lambda}^{\tau} \delta_{\sigma}^{\nu} h^{\mu \pi}+\delta_{\lambda}^{\tau} \delta_{\sigma}^{\mu} h^{\nu \pi}+\delta_{\lambda}^{\pi} \delta_{\sigma}^{\nu} h^{\mu \tau}+\delta_{\lambda}^{\pi} \delta_{\sigma}^{\mu} h^{\nu \tau}\right)\right]
\end{aligned}
$$

As equações de movimento para o campo auxiliar $G_{\mu \nu}^{\lambda}$ podem ser derivadas trivialmente da lagrangiana na forma acima, tal que,

$$
G_{\mu \nu}^{\lambda} \approx\left(M^{-1}\right)_{\mu \nu \pi \tau}^{\lambda \rho}(h) h_{, \rho}^{\pi \tau}
$$


onde a inversa ${ }^{9}$ do tensor definido na Eq. (4.25) é igual a [6]

$$
\begin{aligned}
\left(M^{-1}\right)_{\mu \nu \pi \tau}^{\lambda \rho}(h) & =-\frac{1}{2(d-2)} h^{\lambda \rho} h_{\mu \nu} h_{\pi \tau}+\frac{1}{4} h^{\lambda \rho}\left(h_{\pi \mu} h_{\tau \nu}+h_{\pi \nu} h_{\tau \mu}\right) \\
& -\frac{1}{4}\left(h_{\tau \mu} \delta_{\nu}^{\rho} \delta_{\pi}^{\lambda}+h_{\pi \mu} \delta_{\nu}^{\rho} \delta_{\tau}^{\lambda}+h_{\tau \nu} \delta_{\mu}^{\rho} \delta_{\pi}^{\lambda}+h_{\pi \nu} \delta_{\mu}^{\rho} \delta_{\tau}^{\lambda}\right) .
\end{aligned}
$$

Substituindo a equação de movimento do campo auxiliar, Eq. (4.26), na lagrangiana do formalismo de primeira ordem obtemos que

$$
\mathcal{L}_{\mathrm{EH}}^{\mathrm{I}} \approx-\frac{1}{2 \kappa^{2}} h_{, \lambda}^{\mu \nu}\left(M^{-1}\right)_{\mu \nu \pi \tau}^{\lambda \rho}(h) h_{, \rho}^{\pi \tau}
$$

que é igual a lagrangiana do formalismo de segunda ordem da gravitação na forma dada na Eq. (4.7). O resultado acima deixa claro que equivalência obtida na Eq. (4.23) é assegurada quando o campo auxiliar satisfaz a sua equação de movimento clássica. Logo, podemos reescrever a Eq. (4.28) como

$$
\mathcal{L}_{\mathrm{EH}}^{\mathrm{I}} \approx \mathcal{L}_{\mathrm{EH}}^{\mathrm{II}}
$$

essa igualdade mostra a equivalência clássica entre os formalismos de primeira e segunda ordem da gravitação. Ressaltamos que como na Eq. (2.17), obtida no caso da teoria de YM, a equivalência é obtida quando o campo auxiliar satisfaz sua equação de movimento.

\subsubsection{Quantização}

Assim como não é possível quantizar a gravitação do formalismo usual usando $h^{\mu \nu}$ também não é possível quantizar a lagrangiana do formalismo de primeira ordem diretamente [6]. Precisamos como no formalismo usual usar um campo de fundo, sendo assim definimos novamente $h^{\mu \nu}=$ $\eta^{\mu \nu}+\kappa \phi^{\mu \nu}$ como na Eq. (4.10) e podemos assim continuar com o procedimento básico de quantização. O procedimento de Faddeev-Popov é o mesmo pois a invariância de calibre é a mesma e o determinante de FP não depende do campo auxiliar $G_{\mu \nu}^{\lambda}$. Logo, o setor fantasma é igual em todos formalismos da gravitação somente dependendo do campo do gráviton $\phi^{\mu \nu}$.

Imediatamente definimos o funcional gerador das funções de Green para o formalismo de primeira ordem da gravitação:

$$
Z_{\mathrm{EH}}^{\mathrm{I}}[j, J]=\int \mathcal{D} d_{\nu} \mathcal{D} \bar{d}_{\mu} \mathcal{D} G_{\mu \nu}^{\lambda} \mathcal{D} \phi^{\mu \nu} \exp i\left[\int d^{d} x\left(\mathcal{L}_{\mathrm{eff}}^{\mathrm{I}}+J_{\lambda}^{\mu \nu} G_{\mu \nu}^{\lambda}+j_{\mu \nu} \phi^{\mu \nu}\right)\right],
$$

onde a lagrangiana efetiva para o formalismo de primeira ordem é igual a

$$
\mathcal{L}_{\text {eff }}^{\mathrm{I}}=\frac{1}{2 \kappa^{2}} G_{\mu \nu}^{\lambda} M_{\lambda \rho}^{\mu \nu \pi \tau}(h) G_{\pi \tau}^{\rho}-\frac{1}{\kappa^{2}} G_{\mu \nu}^{\lambda} h_{, \lambda}^{\mu \nu}-\frac{1}{2 \alpha}\left(\partial_{\mu} \phi^{\mu \nu}\right)^{2}+\mathcal{L}_{\text {gh }}(\phi) .
$$

Usando a linearidade do tensor definido na Eq. (4.25):

$$
M(h)=M(\eta)+\kappa M(\phi)
$$

na Eq. (4.31) obtemos

$$
\begin{aligned}
\mathcal{L}_{\text {eff }}^{\mathrm{I}} & =\frac{1}{2 \kappa^{2}} G_{\mu \nu}^{\lambda} M_{\lambda}^{\mu \nu \pi \tau}(\eta) G_{\pi \tau}^{\rho}-\frac{1}{\kappa^{2}} G_{\mu \nu}^{\lambda} \phi_{, \lambda}^{\mu \nu}-\frac{1}{2 \alpha}\left(\partial_{\mu} \phi^{\mu \nu}\right)^{2}+\bar{d}_{\mu} \partial^{2} \eta^{\mu \nu} d_{\nu} \\
& +\frac{1}{2 \kappa} G_{\mu \nu}^{\lambda} M_{\lambda}^{\mu \nu \pi \tau}(\phi) G_{\pi \tau}^{\rho}+\kappa \bar{d}_{\mu}\left[\left(\phi_{, \rho}^{\rho \sigma}\right) \partial_{\sigma} \eta^{\mu \nu}-\left(\phi_{, \rho}^{\rho \mu}\right) \partial^{\nu}+\phi^{\rho \sigma} \partial_{\rho} \partial_{\sigma} \eta^{\mu \nu}-\left(\partial_{\rho} \partial^{\nu} \phi^{\rho \mu}\right)\right] d_{\nu} .
\end{aligned}
$$

\footnotetext{
${ }^{9}$ No espaço em questão $\left(M^{-1}\right)_{\mu \nu \pi \tau}^{\lambda} \underset{\rho}{\rho} M_{\rho}^{\pi \tau \alpha \beta}=\left(\delta_{\mu}^{\alpha} \delta_{\nu}^{\beta}+\delta_{\mu}^{\beta} \delta_{\nu}^{\alpha}\right) \delta_{\gamma}^{\lambda} / 2$.
} 
Na primeira linha da equação acima estão os termos bilineares nos campos que definem os propagadores dos campos $\phi^{\mu \nu}, G_{\mu \nu}^{\lambda}$ e o propagador do campo fantasma. Note que temos um acoplamento entre os campos de calibre e o campo auxiliar, como na teoria de YM, portanto também temos propagadores mistos: $\left\langle\phi^{\mu \nu} G_{\alpha \beta}^{\lambda}\right\rangle$ e $\left\langle G_{\alpha \beta}^{\lambda} \phi^{\mu \nu}\right\rangle$. Já na segunda linha temos os termos de interação que levam no vértice $(G G \phi)$, sem dependência em derivadas, e no vértice do campo fantasma $\left(\bar{d}_{\mu} \phi d_{\nu}\right)$.

Comparando com o formalismo de segunda ordem vemos uma simplificação muito maior do que no caso da teoria de YM, já que passamos de um número infinito de vértice para apenas um mero vértice cúbico sem dependência de momento. Embora tenhamos agora propagadores mistos para compensar. As mesmas dificuldades ligadas a presença dos propagadores mistos que discutimos anteriormente no caso da teoria de YM continuam valendo no caso da gravitação.

Contundo, assim como na teoria de YM também podemos diagonalizar a formulação de primeira ordem da gravitação. Na gravitação a formulação diagonal é mais interessante pois a simplificação ainda é muito relevante, onde temos apenas três vértices cúbicos em comparação aos infinitos vértices da formulação de segunda ordem. Isso torna o formalismo diagonal em uma ferramenta muito útil no estudo perturbativo da gravitação.

As regras de Feynman do formalismo de primeira ordem são derivadas com alguns detalhes em [5], já no apêndice $\mathrm{C}$ damos, como referência, essas regras de Feynman de acordo com as nossas convenções.

\subsection{Formalismo diagonal}

Nessa seção vamos estudar a formulação diagonal do formalismo de primeira ordem no contexto da gravitação. Mostraremos que os infinitos vértices do formalismo usual também se reduzem a um número finito de vértices como no formalismo de primeira ordem, além de removermos os propagadores mistos.

Para obter a forma diagonal da lagrangiana do formalismo de primeira ordem na Eq. (4.24) fazemos a substituição [6]

$$
G_{\mu \nu}^{\lambda}=H_{\mu \nu}^{\lambda}+\left(M^{-1}\right)_{\mu \nu \pi \tau}^{\lambda \rho}(\eta) h_{, \rho}^{\pi \tau}
$$

onde $\left(M^{-1}\right)_{\mu \nu \pi \tau}^{\lambda} \rho(h)$ é definido na Eq. (4.27). Assim temos como resultado

$$
\begin{aligned}
\mathcal{L}_{\mathrm{EH}}^{\mathrm{Id}} & =\frac{1}{2 \kappa^{2}} H_{\mu \nu}^{\lambda} M_{\lambda \rho}^{\mu \nu \pi \tau}(\eta) H_{\pi \tau}^{\rho}-\frac{1}{2} \phi_{, \lambda}^{\mu \nu}\left(M^{-1}\right)_{\mu \nu \pi \tau}^{\lambda \rho}(\eta) \phi_{, \rho}^{\pi \tau} \\
& +\frac{1}{2 \kappa}\left[H_{\mu \nu}^{\lambda}+\kappa \phi_{, \rho}^{\alpha \beta}\left(M^{-1}\right)_{\alpha \beta \mu \nu}^{\rho}{ }_{\alpha \mu}^{\lambda}(\eta)\right] M_{\lambda}^{\mu \nu \pi \tau}(\phi)\left[H_{\pi \tau}^{\sigma}+\kappa\left(M^{-1}\right)_{\pi \tau \gamma \delta}^{\sigma} v(\eta) \phi_{, v}^{\gamma \delta}\right]
\end{aligned}
$$

A demonstração é trivial, onde só usamos, no final, $M(\eta+\kappa \phi)=M(\eta)+\kappa M(\phi)$ para separar os termos bilineares e de interação. Como no formalismo de primeira ordem consideraremos os campos $\phi^{\mu \nu}$ e $H_{\mu \nu}^{\lambda}$ independentemente, onde o campo $H_{\mu \nu}^{\lambda}$ tem o papel de campo auxiliar assim como $G_{\mu \nu}^{\lambda}$.

Para mostrar a equivalência clássica pode-se obter as equações de movimento para o campo auxiliar $H_{\mu \nu}^{\lambda}$ através das equações de Euler-Lagrange usando a lagrangiana dada na Eq. (4.35). 
Contundo é mais fácil usar a Eq. (4.34) que relaciona classicamente os dois campos. Assim substituindo a equação de movimento de campo $G_{\mu \nu}^{\lambda}$ dada pela Eq. (4.26) na Eq. (4.34) obtemos

$$
H_{\mu \nu}^{\lambda} \approx \kappa\left[M^{-1}(\eta+\kappa \phi)-M^{-1}(\eta)\right]_{\mu \nu \pi \tau}^{\lambda \rho} \phi_{, \rho}^{\pi \tau}
$$

que é a equação de movimento para o campo auxiliar ${ }^{10} H_{\mu \nu}^{\lambda}$. Usando a relação (4.36) na lagrangiana do formalismo diagonal obtemos

$$
\mathcal{L}_{\mathrm{EH}}^{\mathrm{Id}} \approx-\frac{1}{2 \kappa^{2}} h_{, \lambda}^{\mu \nu}\left(M^{-1}\right)_{\mu \nu \pi \tau}^{\lambda} \rho(h) h_{, \rho}^{\pi \tau}
$$

que é igual a lagrangiana obtida na Eq. (4.28). Pela Eq. (4.29):

$$
\mathcal{L}_{\mathrm{EH}}^{\mathrm{I}} \approx \mathcal{L}_{\mathrm{EH}}^{\mathrm{II}} \approx \mathcal{L}_{\mathrm{EH}}^{\mathrm{I} d}
$$

mostrando que todas as formulações consideradas nesse trabalho para a gravitação são equivalentes entre si no nível clássico. Além disso, a equivalência é obtida quando os campos auxiliares satisfazem suas equações de movimento mostrando que a condição Eq. (3.7) é válida na gravitação e pode ser usada para estender esses resultados para o âmbito quântico onde também precisamos considerar fontes.

Para a quantização do formalismo diagonal precisamos considerar a lagrangiana efetiva obtida pelo procedimento de Faddeev-Popov que é semelhante a Eq. (4.13) onde adicionamos os termos relativos à fixação de calibre e dos campos fantasmas:

$$
\mathcal{L}_{\text {eff }}^{\mathrm{Id}}=\mathcal{L}_{\mathrm{EH}}^{\mathrm{Id}}+\mathcal{L}_{\text {gf }}(\phi)+\mathcal{L}_{\text {gh }}(\phi)
$$

O funcional gerador para o formalismo diagonal pode ser obtido imediatamente usando a lagrangiana efetiva acima:

$$
Z_{\mathrm{EH}}^{\mathrm{Id}}[j, J]=\int \mathcal{D} d_{\nu} \mathcal{D} \bar{d}_{\mu} \mathcal{D} H_{\mu \nu}^{\lambda} \mathcal{D} \phi^{\mu \nu} \exp i \int d^{d} x\left(\mathcal{L}_{\mathrm{eff}}^{\mathrm{Id}}+J_{\lambda}^{\mu \nu} H_{\mu \nu}^{\lambda}+j_{\mu \nu} \phi^{\mu \nu}\right) .
$$

Pela lagrangiana do formalismo diagonal dada na Eq. (4.35) vemos que os campos $\phi^{\mu \nu}$ e o campo auxiliar $H_{\mu \nu}^{\lambda}$ não estão mais acoplados, ou seja, não temos mais propagadores mistos. Por outro lado, além do vértice $(H H \phi)$, igual ao vértice $(G G \phi)$, temos os vértices $(G \phi \phi)$ e $(\phi \phi \phi)^{11}$ que possuem uma dependência de momento. Ainda temos uma simplificação imensa já que o número de vértice continua finito como no formalismo de primeira ordem.

No próximo capítulo vamos mostrar como estender a Eq. (4.38) que mostra a equivalência clássica entre os formalismos da gravitação para o âmbito quântico de forma análoga ao que já fizemos para a teoria de YM.

\footnotetext{
${ }^{10}$ A equação de movimento obtida na Eq. (4.36) concorda com o resultado obtido na Ref. [16] que parte da equação de Euler-Lagrange. A concordância é a menos de um fator de $\kappa$ que não aparece na Ref. [16, eq. 3.10] devido a um pequeno erro onde foi considerado que $h_{, \lambda}^{\mu \nu}=\phi_{, \lambda}^{\mu \nu}$.

${ }^{11}$ Conforme [16], esse vértice coincide com o vértice cúbico do formalismo de segunda ordem. Assim como no caso da teoria de YM essa equivalência é esperada já que mostraremos que os formalismos são equivalentes no âmbito quântico e além das amplitudes on-shell, as funções de Green (amputadas) para o campo $\phi^{\mu \nu}$ também são as mesmas em todos formalismos.
} 


\subsubsection{Regras de Feynman}

Nessa seção listamos as regras (momentos entrando nos vértices) obtidas no apêndice C:
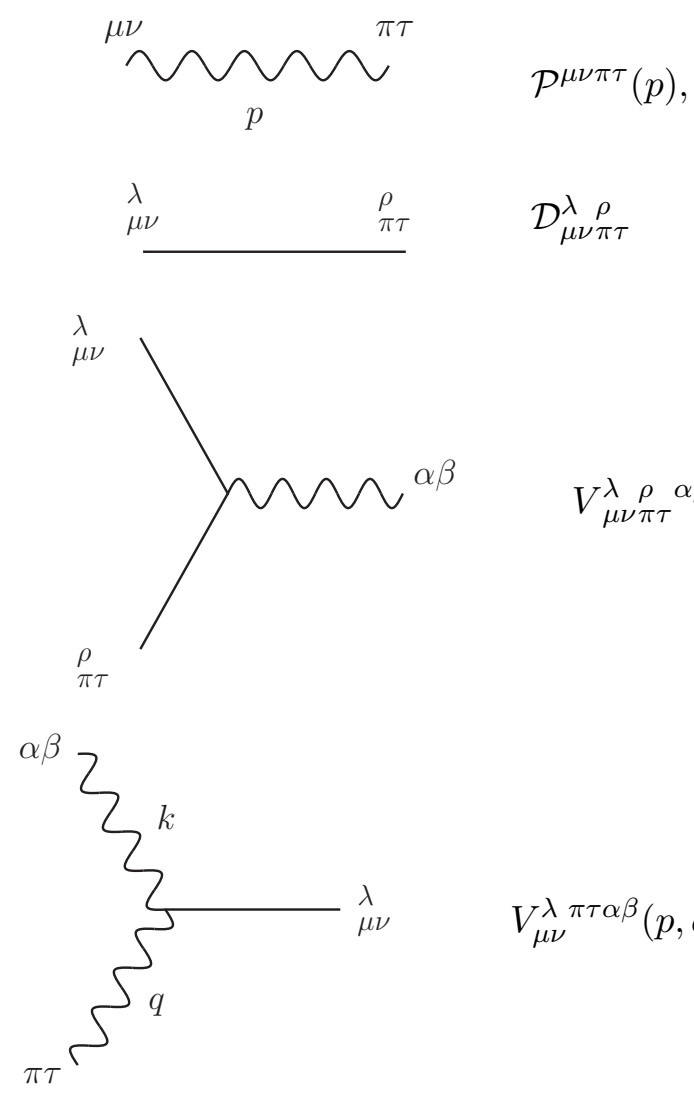

$$
V_{\mu \nu \pi \tau}^{\lambda \rho \alpha \beta}
$$

$$
V_{\mu \nu}^{\lambda \pi \tau \alpha \beta}(p, q, k)
$$
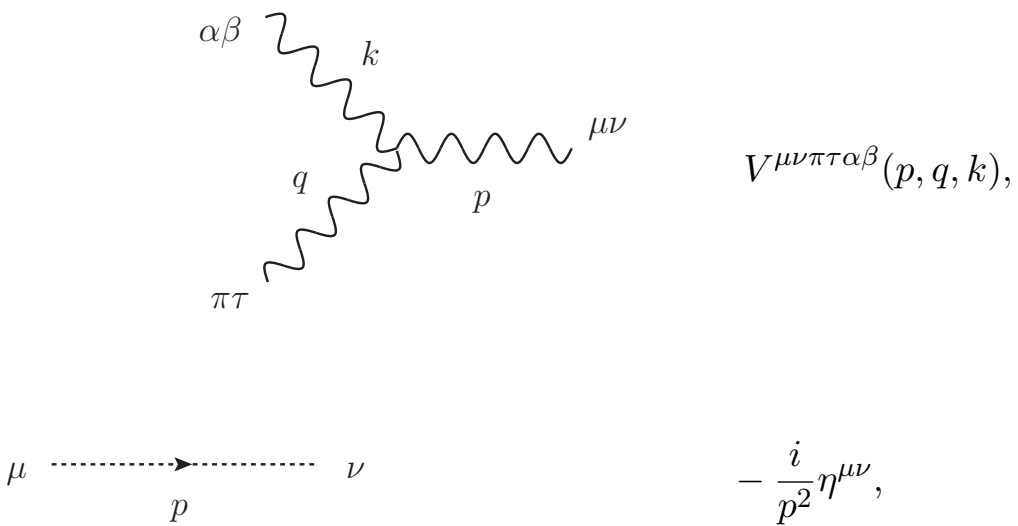

$$
-\frac{i}{p^{2}} \eta^{\mu \nu}
$$

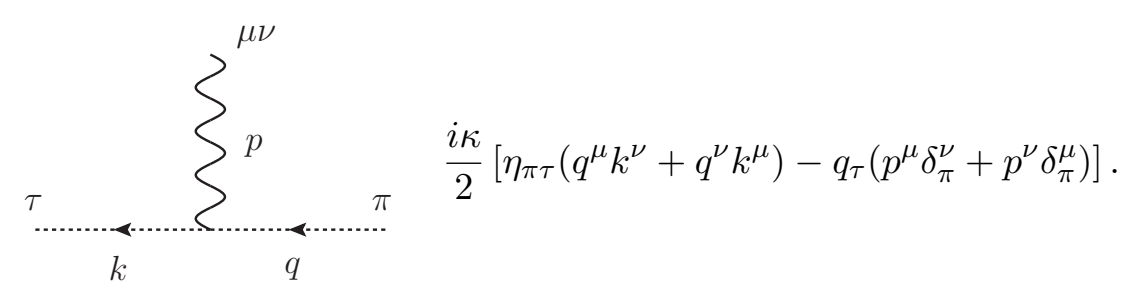

Linhas onduladas, sólidas e tracejadas representam, respectivamente, os campos $\phi^{\mu \nu}, H_{\mu \nu}^{\lambda}$ e os campos fantasmas $d_{\nu}, \bar{d}_{\mu}$. As expressões explícitas para $\mathcal{P}^{\mu \nu \pi \tau}(p), \mathcal{D}_{\mu \nu \pi \tau}^{\lambda \rho}, V_{\mu \nu \pi \tau}^{\lambda \rho}{ }^{\alpha \beta}, V_{\mu \nu}^{\lambda \pi \tau \alpha \beta}(p, q, k)$, $V^{\mu \nu \pi \tau \alpha \beta}(p, q, k)$ são dadas nas Eqs. (C.55), (C.56), (C.57), (C.58), (C.59). 



\section{CAPÍTULO 5}

\section{Equivalência QuÂNTICA: Gravitação}

Neste capítulo demonstraremos a equivalência quântica das formulações da gravitação que vimos no capítulo anterior. Deduziremos identidades estruturais entre as funções de Green computadas na formulações de primeira ordem (e diagonal) e as funções de Green computadas na formulação usual de segunda ordem. Esse capítulo, em sua maioria, é inspirado no artigo [16] que conforme já mencionado foi publicado durante esse trabalho.

Demostraremos que o formalismo de primeira ordem e o formalismo diagonal são equivalentes no nível quântico ao formalismo de segunda ordem da gravitação sem campos de matéria ${ }^{1}$ implicando na equivalência quântica de todas as formulações consideradas para gravitação.

Primeiramente, consideraremos a demonstração da equivalência quântica, sem fontes, para o formalismo de primeira ordem conforme visto em [6]. E então mostraremos que podemos considerar a condição geral (3.7) (adaptada para gravitação) para obter a equivalência quântica concordando com a abordagem do artigo [16].

\subsection{Equivalência parcial para o formalismo de pri- meira ordem}

Aqui vamos mostrar a equivalência quântica, sem fontes, entre os formalismos de primeira ordem e segunda ordem da gravitação. Embora busquemos primeiramente estender a equivalência clássica ao nível quântico sem as fontes, essa análise fundamenta o ansatz ${ }^{2}$, dado genericamente pela Eq. (3.7), do shift que usamos para demonstrar a equivalência quântica com as fontes.

\footnotetext{
${ }^{1}$ Conforme $[4,13]$, o acoplamento de campos de matéria com a gravitação, como férmions, leva ao surgimento de torção, sendo assim os formalismos de primeira e segunda ordem deixam de ser equivalentes no nível clássico. Mais recentemente, em [35], mostrou-se que para o acoplamento de um campo de matéria que não depende da conexão a equivalência ainda é válida. Já em [36, 37] encontra-se abordagens onde temos a inclusão de férmions com certas modificações ao formalismo de primeira ordem.

${ }^{2}$ Além disso, já que estamos assumindo que podemos normalizar o funcional gerador $(Z[0]=1)$ é importante mostrar a equivalência dos funcionais geradores sem fontes para garantir que não há inconsistências.
} 
Consideremos o funcional gerador do formalismo de primeira ordem sem fontes:

$$
Z_{\mathrm{EH}}^{\mathrm{I}}[0] \equiv Z_{\mathrm{EH}}^{\mathrm{I}}[0,0]=\int \mathcal{D} d_{\nu} \mathcal{D} \bar{d}_{\mu} \mathcal{D} G_{\mu \nu}^{\lambda} \mathcal{D} \phi^{\mu \nu} \exp i\left[\int d^{d} x\left(\mathcal{L}_{\mathrm{EH}}^{\mathrm{I}}+\mathcal{L}_{\mathrm{gf}}(\phi)+\mathcal{L}_{\mathrm{gh}}(\phi)\right)\right] .
$$

Podemos generalizar a Eq. (3.7), tal que, para a integração do campo auxiliar podemos fazer um shift na Eq. (5.1) igual a

$$
G_{\mu \nu}^{\lambda}=\tilde{G}_{\mu \nu}^{\lambda}+S_{\mu \nu}^{\lambda}
$$

onde $G_{\mu \nu}^{\lambda} \approx S_{\mu \nu}^{\lambda}$. Ou seja, $S_{\mu \nu}^{\lambda}$ é dado pela configuração clássica do campo auxiliar, nesse caso $S_{\mu \nu}^{\lambda}$ é dado pela Eq. (4.26). Pelo ansatz acima, o shift seria igual a

$$
G_{\mu \nu}^{\lambda}=\tilde{G}_{\mu \nu}^{\lambda}+\left(M^{-1}\right)_{\mu \nu \pi \tau}^{\lambda \rho}(h) h_{, \rho}^{\pi \tau}
$$

que concorda com [6, eq. 3.14].

Substituindo o shift (5.3) no funcional gerador (5.1) obtemos

$$
\begin{aligned}
Z_{\mathrm{EH}}^{\mathrm{I}}[0] & =\int \mathcal{D} d_{\nu} \mathcal{D} \bar{d}_{\mu} \mathcal{D} \tilde{G}_{\mu \nu}^{\lambda} \mathcal{D} \phi^{\mu \nu} \exp i\left[\int d ^ { d } x \left(\frac{1}{2 \kappa^{2}} \tilde{G}_{\mu \nu}^{\lambda} M_{\lambda}^{\mu \nu \pi \tau}(h) \tilde{G}_{\pi \tau}^{\rho}\right.\right. \\
& \left.\left.-\frac{1}{2 \kappa^{2}} h_{, \lambda}^{\mu \nu}\left(M^{-1}\right)_{\mu \nu \pi \tau}^{\lambda \rho}(\eta) h_{, \rho}^{\pi \tau}+\mathcal{L}_{\mathrm{gf}}(\phi)+\mathcal{L}_{\mathrm{gh}}(\phi)\right)\right]
\end{aligned}
$$

onde identificamos a lagrangiana do formalismo de segunda ordem, tal que, podemos reescrever o funcional gerador acima como

$$
Z_{\mathrm{EH}}^{\mathrm{I}}[0]=\int \mathcal{D} d_{\nu} \mathcal{D} \bar{d}_{\mu} \mathcal{D} \tilde{G}_{\mu \nu}^{\lambda} \mathcal{D} \phi^{\mu \nu} \exp i\left[\int d^{d} x\left(\frac{1}{2 \kappa^{2}} \tilde{G}_{\mu \nu}^{\lambda} M_{\lambda}^{\mu \nu \pi \tau}(h) \tilde{G}_{\pi \tau}^{\rho}+\mathcal{L}_{\mathrm{eff}}^{\mathrm{II}}\right)\right] .
$$

O resultado acima é análogo ao obtido na Eq. (3.4), por outro lado, diferentemente da Eq. (3.4), na Eq. (5.5) o termo do campo auxiliar não é uma mera constante que pode ser descartada imediatamente.

Porém, conforme [6], as contribuições perturbativas devido ao termo que depende do campo auxiliar na Eq. (5.5) se anulam usando regularização dimensional, pois esse termo leva a diagramas com loops, tais que, ao menos um dos propagadores que compõe o loop seja o propagador do campo $\tilde{G}_{\mu \nu}^{\lambda}$. Porém, note que tanto o propagador do campo $\tilde{G}_{\mu \nu}^{\lambda}$ quanto o vértice $(G G \phi)$ não dependem de momento, logo as integrais desses loops tem a forma geral [6]

$$
\int d^{d} p P\left(p^{\mu}\right)
$$

onde $P\left(p^{\mu}\right)$ é um polinômio no momento interno. No método de regularização usado nesse trabalho, a regularização dimensional, tais integrais [38, 39] são nulas. Sendo assim, usando regularização dimensional, o termo que depende do campo $\tilde{G}_{\mu \nu}^{\lambda}$ na Eq. (5.5) não contribui e pode ser descartado.

Ou mais diretamente, pela integração do campo $\tilde{G}_{\mu \nu}^{\lambda}$ na Eq. (5.5) obtemos o fator de

$$
\operatorname{det}[M(h)]^{-1 / 2}=\operatorname{det}[M(\eta)+k M(\phi)]^{-1 / 2} .
$$

A expansão desse determinante resulta numa série de potência em $\kappa$ que é igual a soma de diagramas não massivo do tipo "tadpole" [40]. Tais diagramas vão a zero quando usamos regularização dimensional, logo a contribuição resultante é igual a $\operatorname{det}[M(\eta)]^{-1 / 2}$, uma constante, que é então absorvida na normalização do funcional gerador. 
Ressaltamos que diferentemente do caso análogo na teoria de YM onde a integração do campo auxiliar na Eq. (3.4) leva a uma constante irrelevante, na gravitação temos um termo não trivial que depende do campo de calibre e só se reduz a uma constante usando regularização dimensional. Para casos mais gerais onde diagramas do tipo "tadpole", como na teoria de campos à temperatura finita, não se anulam a equivalência pode deixar de ser válida.

Porém, para todos efeitos nesse trabalho usamos a regularização dimensional, tal que, podemos afirmar que

$$
Z_{\mathrm{EH}}^{\mathrm{I}}[0]=\int \mathcal{D} d_{\nu} \mathcal{D} \bar{d}_{\mu} \mathcal{D} \phi^{\mu \nu} \exp i \int d^{d} x \mathcal{L}_{\mathrm{eff}}^{\mathrm{II}}=Z_{\mathrm{EH}}^{\mathrm{II}}[0] .
$$

A equação acima mostra que os formalismo de primeira ordem e de segunda ordem para gravitação são parcialmente equivalentes no nível quântico.

No caso geral com fontes continuaremos usando o ansatz discutindo na seção 3.1 que no caso da gravitação toma a forma do shift dado na Eq. (5.3). De forma genérica a condição para o shift é análogo a condição (3.7), tal que, na gravitação

$$
\tilde{G}_{\mu \nu}^{\lambda}=G_{\mu \nu}^{\lambda}-S_{\mu \nu}^{\lambda} \approx 0 \Leftrightarrow G_{\mu \nu}^{\lambda} \approx S_{\mu \nu}^{\lambda}
$$

onde $G_{\mu \nu}^{\lambda}$ é um campo auxiliar qualquer.

\subsection{Equivalência quântica para o formalismo de Pala- tini}

Nessa seção vamos generalizar a equivalência quântica entre os formalismo de primeira ordem e segunda ordem considerando as fontes. Conforme vimos na seção 3.2 quando temos fontes a configuração clássica do campo auxiliar também precisa levar em consideração tais termos, para isso podemos usar as fontes adicionadas durante a quantização como fontes clássicas na equação de Euler-Lagrange.

Por isso, em vez da lagrangiana da Eq. (4.24) usaremos ${ }^{3}$

$$
\mathcal{L}_{\mathrm{EH}}^{\mathrm{I}}[j, J]=\mathcal{L}_{\mathrm{EH}}^{\mathrm{I}}+J_{\lambda}^{\mu \nu} G_{\mu \nu}^{\lambda}+j_{\mu \nu} \phi^{\mu \nu} .
$$

Comparando a lagrangiana com fontes com a lagrangiana sem fontes dada na Eq. (4.24) vemos que fazendo a substituição

$$
h_{, \lambda}^{\mu \nu} \rightarrow h_{, \lambda}^{\mu \nu}-\kappa^{2} J_{\lambda}^{\mu \nu}
$$

na Eq. (4.24) obtemos a Eq. (5.10) a menos do termo $j_{\mu \nu} \phi^{\mu \nu}$. No entanto, esse termo não depende do campo auxiliar e não contribui para a equação de movimento do campo auxiliar, logo pode-se derivar a equação de movimento para o campo auxiliar com fontes a partir da Eq. (4.26) pela a substituição (5.11), tal que

$$
G_{\mu \nu}^{\lambda} \approx\left(M^{-1}\right)_{\mu \nu \pi \tau}^{\lambda \rho}(h)\left(h_{, \rho}^{\pi \tau}-\kappa^{2} J_{\rho}^{\pi \tau}\right),
$$

\footnotetext{
${ }^{3}$ Embora a lagrangiana (sem fontes) mais adequada fosse a lagrangiana efetiva, com os termos de fixação de calibre e dos campos fantasmas, não há diferença na obtenção das equações de movimento para o campo auxiliar já que os termos extras só dependem do campo de calibre.
} 
onde $h^{\mu \nu}=\eta^{\mu \nu}+\kappa \phi^{\mu \nu}$.

Assim pela condição (5.9) para integrar o campo auxiliar na Eq. (4.30) podemos fazer o shift

$$
\tilde{G}_{\mu \nu}^{\lambda}=G_{\mu \nu}^{\lambda}+\left(M^{-1}\right)_{\mu \nu \pi \tau}^{\lambda \rho}(h)\left(h_{, \rho}^{\pi \tau}-\kappa^{2} J_{\rho}^{\pi \tau}\right)
$$

obtendo $^{4}$

$$
\begin{aligned}
Z_{\mathrm{EH}}^{\mathrm{I}}[j, J] & =\int \mathcal{D} d_{\nu} \mathcal{D} \bar{d}_{\mu} \mathcal{D} \tilde{G}_{\mu \nu}^{\lambda} \mathcal{D} \phi^{\mu \nu} \exp i\left[\int d ^ { d } x \left(\mathcal{L}_{\mathrm{eff}}^{\mathrm{II}}+\frac{1}{2 \kappa^{2}} \tilde{G}_{\mu \nu}^{\lambda} M_{\lambda}^{\mu \nu \pi \tau}(h) \tilde{G}_{\pi \tau}^{\rho}\right.\right. \\
& \left.\left.+J_{\lambda}^{\mu \nu}\left(M^{-1}\right)_{\mu \nu \pi \tau}^{\lambda \rho}(h) h_{, \rho}^{\pi \tau}-\frac{\kappa^{2}}{2} J_{\lambda}^{\mu \nu}\left(M^{-1}\right)_{\mu \nu \pi \tau}^{\lambda \rho}(h) J_{\rho}^{\pi \tau}+j_{\mu \nu} \phi^{\mu \nu}\right)\right] .
\end{aligned}
$$

Antes de integramos o campo auxiliar é interessante mencionar que o funcional gerador acima é análogo ao funcional obtido na Eq. (3.11), onde temos uma dependência semelhante na fonte do campo auxiliar.

Para ver a razão dessa semelhança note que podemos escrever os termos com dependência na fonte de forma genérica como

$$
J_{\lambda}^{\mu \nu} S_{\mu \nu}^{\lambda}+\frac{1}{2 \kappa^{2}} S_{\mu \nu}^{\lambda}[J] M_{\lambda \rho}^{\mu \nu \pi \tau}(h) S_{\pi \tau}^{\rho}[J]
$$

onde $S_{\mu \nu}^{\lambda}$ é definido pela condição $(5.9)$ e $S_{\mu \nu}^{\lambda}[J]$ é somente a parte que depende da fonte $J$ de $S_{\mu \nu}^{\lambda}$. Além do termo acoplando a configuração clássica do campo auxiliar com a fonte $J$, se $S[J] \neq 0$ é esperado uma dependência quadrática em $J$.

Continuando, podemos integrar o campo auxiliar $\tilde{G}_{\mu \nu}^{\lambda}$ na Eq. (5.14) como na seção anterior chegando no mesmo determinante da Eq. (5.7). Conforme os argumentos mencionados esse determinante pode ser descartado usando regularização dimensional, tal que, o funcional gerador da Eq. (5.14) pode ser escrito simplesmente como

$$
\begin{aligned}
Z_{\mathrm{EH}}^{\mathrm{I}}[j, J] & =\int \mathcal{D} d_{\nu} \mathcal{D} \bar{d}_{\mu} \mathcal{D} \phi^{\mu \nu} \exp i\left[\int d ^ { d } x \left(\mathcal{L}_{\mathrm{eff}}^{\mathrm{II}}+j_{\mu \nu} \phi^{\mu \nu}\right.\right. \\
& \left.\left.+J_{\lambda}^{\mu \nu}\left(M^{-1}\right)_{\mu \nu \pi \tau}^{\lambda \rho}(h) h_{, \rho}^{\pi \tau}-\frac{\kappa^{2}}{2} J_{\lambda}^{\mu \nu}\left(M^{-1}\right)_{\mu \nu \pi \tau}^{\lambda \rho}(h) J_{\rho}^{\pi \tau}\right)\right]
\end{aligned}
$$

onde $\mathcal{L}_{\text {eff }}^{\text {II }}$ é lagrangiana efetiva do formalismo de segunda ordem da gravitação definida na Eq. (4.13). Assim é evidente que o funcional gerador do formalismo de primeira ordem na forma alternativa dada acima é equivalente ao formalismo de segunda ordem.

Mais claramente, para $J=0$, temos pela a Eq. (5.16) que

$$
Z_{\mathrm{EH}}^{\mathrm{I}}[j, 0]=Z_{\mathrm{EH}}^{\mathrm{II}}[j]
$$

Ou seja, os formalismos de primeira ordem e segunda ordem são equivalentes no nível quântico. Consequentemente pela Eq. (5.17) temos que o formalismo de primeira ordem leva nas mesmas

\footnotetext{
${ }^{4}$ A derivação é trivial usando uma formulação matricial. Assim, o shift da Eq. (5.13) tomaria a forma algébrica $\tilde{G}=G+M^{-1}\left(\partial \cdot h-\kappa^{2} J\right)=G+\left(\partial \cdot h-\kappa^{2} J\right) M^{-1}$, onde usamos o fato que $M$ é simétrica $M_{a b}=M_{b a}$. Os índices $a$ e $b$ representam um conjunto de três índices como os que aparecem na definição dos elementos de $M$ na Eq. (4.25). Logo, escrevendo a lagrangiana da Eq. (5.10) na forma matricial como $G M G /\left(2 \kappa^{2}\right)-G \partial \cdot h / \kappa^{2}+J G+j \phi$ e aplicando o shift como escrevemos anteriormente obtemos o resultado visto na Eq. (5.14) em sua forma matricial.
} 
funções de Green para o campo de calibre $\phi^{\mu \nu}$ que o formalismo de segunda ordem:

$$
\begin{aligned}
\left\langle 0\left|T \phi^{\mu_{1} \nu_{1}}\left(x_{1}\right) \cdots \phi^{\mu_{n} \nu_{n}}\left(x_{n}\right)\right| 0\right\rangle & \left.\equiv(-i)^{n} \frac{\delta^{n} Z_{\mathrm{EH}}^{\mathrm{II}}[j]}{\delta j_{\mu_{1} \nu_{1}}\left(x_{1}\right) \cdots \delta j_{\mu_{n} \nu_{n}}\left(x_{n}\right)}\right|_{j=0} \\
& =\left.(-i)^{n} \frac{\delta^{n} Z_{\mathrm{EH}}^{\mathrm{I}}[j, J]}{\delta j_{\mu_{1} \nu_{1}}\left(x_{1}\right) \cdots \delta j_{\mu_{n} \nu_{n}}\left(x_{n}\right)}\right|_{J=j=0} .
\end{aligned}
$$

Para $J \neq 0$, o lado direito da Eq. (5.26) pode ser interpretado como uma generalização do funcional gerador do formalismo de segunda ordem dado na Eq. (4.14). Além de gerar funções de Green para o campo de calibre $\phi^{\mu \nu}$ também gera correlações para o operador composto $\left[M^{-1}[h(x)]-M^{-1}(\eta)\right]_{\mu \nu \pi \tau}^{\lambda} h_{, \rho}^{\pi \tau}(x)$.

Como na seção 3.2, a comparação dessas duas formas do funcional gerador do formalismo de primeira ordem leva a identidades estruturais que relacionam funções de Green no formalismo de primeira ordem com correlações relacionadas ao campo composto $\left.\left(M^{-1}\right)_{\mu \nu \pi \tau}^{\lambda} \rho h(x)\right] h_{, \rho}^{\pi \tau}(x)$. Pode-se deduzir pela essa análise que

$$
\left\langle 0\left|T G_{\mu \nu}^{\lambda}(x) \phi^{\alpha \beta}(y)\right| 0\right\rangle=\kappa\left\langle 0\left|T\left(M^{-1}\right)_{\mu \nu \pi \tau}^{\lambda \rho}[\eta+\kappa \phi(x)] \phi_{, \rho}^{\pi \tau}(x) \phi^{\alpha \beta}(y)\right| 0\right\rangle
$$

e

$$
\begin{aligned}
& \left\langle 0\left|T G_{\mu \nu}^{\lambda}(x) G_{\alpha \beta}^{\gamma}(y)\right| 0\right\rangle=i \kappa^{2}\left\langle 0\left|T\left(M^{-1}\right)_{\mu \nu \alpha \beta}^{\lambda \gamma}[\eta+\kappa \phi(x)]\right| 0\right\rangle \delta(x-y) \\
& +\kappa^{2}\left\langle 0\left|T\left(M^{-1}\right)_{\mu \nu \pi_{2} \tau_{2}}^{\lambda \rho_{2}}[\eta+\kappa \phi(x)] \phi_{, \rho_{2}}^{\pi_{2} \tau_{2}}(x)\left(M^{-1}\right)_{\alpha \beta \pi_{1} \tau_{1}}^{\gamma} \rho_{1}[\eta+\kappa \phi(y)] \phi_{, \rho_{1}}^{\pi_{1} \tau_{1}}(y)\right| 0\right\rangle .
\end{aligned}
$$

As identidades acima, (5.19) e (5.20), são análogas respectivamente as identidades (3.35) e (3.44). Elas mostram, analogamente, uma relação entre os propagadores inteiros no formalismo de primeira ordem com correlações para a configuração clássica do campo auxiliar no formalismo de segunda ordem da gravitação. Assim como a Eq. (3.44) no caso da gravitação, Eq. (5.20), também temos um termo de contato que é essencial para a verificação de tais identidades no nível árvore. Embora a derivação e verificação das identidades (5.19) e (5.20) não sejam consideradas nesse trabalho, iremos ver, em detalhes, a dedução de identidades semelhantes para o formalismo diagonal cuja verificação é realizada perturbativamente até um loop no capítulo 6 .

Na próxima seção demostraremos a equivalência quântica para o formalismo diagonal e o formalismo de segunda ordem conforme é discutido no artigo [16]. Consequentemente, deduziremos algumas identidades estruturais que relacionam as funções de Green do formalismo diagonal com correlações de campos compostos computadas no formalismo de segunda ordem da gravitação.

Para concluir essa seção discutimos uma generalização da Eq. (5.15) que revela uma estrutura geral para a dependência na fonte $J$ após a integração dos campos auxiliares considerados. Essa estrutura é válida tanto na gravitação quanto na teoria de YM mostrando a generalidade do procedimento.

Podemos generalizar a Eq. (5.15) ainda mais ao notar que o segundo termo na mesma é igual ao termo bilinear no campo auxiliar $G_{\mu \nu}^{\lambda}$ fazendo $G_{\mu \nu}^{\lambda} \rightarrow S_{\mu \nu}^{\lambda}[J]$. Isso induz generalizar a Eq. (5.15) como $^{5}$

$$
\frac{1}{2} S[J] \cdot D \cdot S[J]+J \cdot S
$$

\footnotetext{
${ }^{5}$ Usamos uma notação genérica para produto já que estamos considerando um espaço tensorial geral. Por exemplo, no caso da gravitação $(M(h) \cdot S)_{\lambda}^{\mu \nu} \equiv M_{\lambda}^{\mu \nu \pi \tau}(h) S_{\pi \tau}^{\rho}$. Note que a operação $(\cdot)$ geralmente não é comutativa, sendo assim $A \cdot B$ usualmente é diferente de $B \cdot A$.
} 
onde $S[J]$ é definido como a parte que dependente da fonte $J$ da configuração clássica do campo auxiliar $S$. Já $D$ pode ser obtido ${ }^{6}$ pela segunda derivada da parte bilinear nos campos da lagrangiana em relação ao campo auxiliar. A Eq. (5.21) nos dá diretamente a dependência de $J$ obtida pelo shift que satisfaz a condição (5.9) (ou a condição (3.7) no caso da teoria de YM).

Para esclarecer consideremos, por exemplo, o caso do formalismo de primeira ordem da teoria de YM. Pela Eq. (3.17): $S[J]=-2 J^{a \mu \nu}$ e $D=-D_{\mu \nu \alpha \beta}=-I_{\mu \nu \alpha \beta} / 2$, tal que, a Eq. (5.21) resulta em

$$
2 J \cdot D \cdot J-2 J \cdot J=J \cdot I \cdot J-2 J \cdot J=-J \cdot J
$$

concordando com a dependência obtida nas Eqs. (3.59) e (3.23).

\subsection{Equivalência quântica para o formalismo diagonal}

Nessa seção usaremos a abordagem usada em [16] para demostrar a equivalência quântica entre os formalismo de primeira ordem e segunda ordem considerando as fontes. O caso sem fontes pode ser considerando de forma semelhante tomando o limite $j \rightarrow 0$ e $J \rightarrow 0$.

Fazendo o shift [16]

$$
H_{\mu \nu}^{\lambda} \rightarrow H_{\mu \nu}^{\lambda}+\kappa\left[M^{-1}(\eta+\kappa \phi)-M^{-1}(\eta)\right]_{\mu \nu \pi \tau}^{\lambda \rho} \phi_{, \rho}^{\pi \tau}-\kappa^{2}\left(M^{-1}\right)_{\mu \nu \pi \tau}^{\lambda}(\eta+\kappa \phi) J_{\rho}^{\pi \tau}
$$

no funcional gerador do formalismo definido na Eq. (4.30) obtemos que

$$
\begin{aligned}
& Z_{\mathrm{EH}}^{\mathrm{Id}}=\int \mathcal{D} d_{\nu} \mathcal{D} \bar{d}_{\mu} \mathcal{D} \phi^{\mu \nu} \mathcal{D} H_{\mu \nu}^{\lambda} \exp i \int d^{d} x\left(\frac{1}{2 \kappa^{2}} H_{\mu \nu}^{\lambda}[M(\eta)+\kappa M(\phi)]_{\lambda}^{\mu \nu \pi \tau} H_{\pi \tau}^{\sigma}+\mathcal{L}_{\mathrm{eff}}^{\mathrm{II}}\right. \\
& \left.+\kappa J_{\lambda}^{\mu \nu}\left[M^{-1}(\eta+\kappa \phi)-M^{-1}(\eta)\right]_{\mu \nu \pi \tau}^{\lambda \rho} \phi_{, \rho}^{\pi \tau}-\frac{\kappa^{2}}{2} J_{\lambda}^{\mu \nu}\left(M^{-1}\right)_{\mu \nu \pi \tau}^{\lambda \rho}(\eta+\kappa \phi) J_{\rho}^{\pi \tau}+j_{\mu \nu} \phi^{\mu \nu}\right)
\end{aligned}
$$

onde usamos a notação compacta [16]

$$
\left[M^{-1}(\eta+\kappa \phi)-M^{-1}(\eta)\right]_{\mu \nu \pi \tau}^{\lambda \rho} \equiv\left(M^{-1}\right)_{\mu \nu \pi \tau}^{\lambda} \rho(\eta+\kappa \phi)-\left(M^{-1}\right)_{\mu \nu \pi \tau}^{\lambda \rho}(\eta)
$$

A integral sobre o campo auxiliar $H_{\mu \nu}^{\lambda}$ na Eq. (5.24) resulta no determinante $\operatorname{det}[M(h)]^{-1 / 2}$ que conforme já discutido leva a uma constante usando regularização dimensional. Essa constante é absorvida pela normalização do funcional gerador, tal que, a Eq. (5.24) se reduz a

$$
\begin{array}{r}
Z_{\mathrm{EH}}^{\mathrm{Id}}=\int \mathcal{D} d_{\nu} \mathcal{D} \bar{d}_{\mu} \mathcal{D} \phi^{\mu \nu} \exp i \int d^{d} x\left(\mathcal{L}_{\mathrm{eff}}^{\mathrm{II}}+\kappa J_{\lambda}^{\mu \nu}\left[M^{-1}(\eta+\kappa \phi)-M^{-1}(\eta)\right]_{\mu \nu \pi \tau}^{\lambda \rho} \phi_{, \rho}^{\pi \tau}\right. \\
\left.-\frac{\kappa^{2}}{2} J_{\lambda}^{\mu \nu}\left(M^{-1}\right)_{\mu \nu \pi \tau}^{\lambda \rho}(\eta+\kappa \phi) J_{\rho}^{\pi \tau}+j_{\mu \nu} \phi^{\mu \nu}\right) .
\end{array}
$$

Com $J=0$, a Eq. (5.26) pode se reescrita como

$$
Z_{\mathrm{EH}}^{\mathrm{I}}[j, 0]=Z_{\mathrm{EH}}^{\mathrm{II}}[j]
$$

onde fica evidente que o formalismos diagonal e de segunda ordem são equivalentes ao nível quântico. Em consequência, temos que as funções de Green do campo de calibre $\phi^{\mu \nu}$ no formalismo

\footnotetext{
${ }^{6}$ Podemos identificar $D$ imediatamente escrevendo a parte bilinear no campo auxiliar da lagrangiana como $G \cdot D \cdot G / 2$.
} 
diagonal e no formalismo de segunda ordem da gravitação coincidem, ou seja,

$$
\begin{aligned}
\left\langle 0\left|T \phi^{\mu_{1} \nu_{1}}\left(x_{1}\right) \cdots \phi^{\mu_{n} \nu_{n}}\left(x_{n}\right)\right| 0\right\rangle & \left.\equiv(-i)^{n} \frac{\delta^{n} Z_{\mathrm{EH}}^{\mathrm{II}}[j]}{\delta j_{\mu_{1} \nu_{1}}\left(x_{1}\right) \cdots \delta j_{\mu_{n} \nu_{n}}\left(x_{n}\right)}\right|_{j=0} \\
& =\left.(-i)^{n} \frac{\delta^{n} Z_{\mathrm{EH}}^{\mathrm{Id}}[j, J]}{\delta j_{\mu_{1} \nu_{1}}\left(x_{1}\right) \cdots \delta j_{\mu_{n} \nu_{n}}\left(x_{n}\right)}\right|_{J=j=0} .
\end{aligned}
$$

Já com $J \neq 0$, o lado direito da Eq. (5.16) pode ser interpretado como uma generalização do funcional gerador do formalismo de segunda ordem dado na Eq. (4.14). Além de gerar funções de Green para o campo de calibre $\phi^{\mu \nu}$ também gera correlações para o operador composto

$$
\Delta_{\mu \nu}^{\lambda}(x) \equiv\left[M^{-1}(h)-M^{-1}(\eta)\right]_{\mu \nu \pi \tau}^{\lambda \rho} h_{, \rho}^{\pi \tau}(x)
$$

Na próxima seção vamos considerar as funções de Green com campos auxiliares externos usando as duas formas, (4.30) e (5.26), do funcional gerador do formalismo diagonal deduzindo identidades estruturais.

Antes demostraremos que o shift considerado na Eq. (5.23) respeita a condição (5.9). Partindo da equação de movimento do campo $G_{\mu \nu}^{\lambda}$ com fontes deduzida anteriormente (ver Eq. (5.12)):

$$
G_{\mu \nu}^{\lambda} \approx\left(M^{-1}\right)_{\mu \nu \pi \tau}^{\lambda \rho}(h)\left(h_{, \rho}^{\pi \tau}-\kappa^{2} J_{\rho}^{\pi \tau}\right)
$$

podemos usar a relação clássica entre $G_{\mu \nu}^{\lambda}$ e $H_{\mu \nu}^{\lambda}$ dada por (ver Eq. (4.34))

$$
G_{\mu \nu}^{\lambda}=H_{\mu \nu}^{\lambda}+\left(M^{-1}\right)_{\mu \nu \pi \tau}^{\lambda \rho}(\eta) h_{, \rho}^{\pi \tau},
$$

tal que, a equação de movimento para o campo $H_{\mu \nu}^{\lambda}$ com fonte é igual a

$$
H_{\mu \nu}^{\lambda} \approx\left[M^{-1}(h)-M^{-1}(\eta)\right]_{\mu \nu \pi \tau}^{\lambda \rho} h_{, \rho}^{\pi \tau}-\kappa^{2}\left(M^{-1}\right)_{\mu \nu \pi \tau}^{\lambda \rho}(h) J_{\rho}^{\pi \tau} .
$$

Substituindo a Eq. (5.32) na Eq. (5.9) (onde o campo auxiliar no caso é $H_{\mu \nu}^{\lambda}$ ) obtemos o shift

$$
\tilde{H}_{\mu \nu}^{\lambda}=H_{\mu \nu}^{\lambda}+\left[M^{-1}(h)-M^{-1}(\eta)\right]_{\mu \nu \pi \tau}^{\lambda \rho} h_{, \rho}^{\pi \tau}-\kappa^{2}\left(M^{-1}\right)_{\mu \nu \pi \tau}^{\lambda \rho}(h) J_{\rho}^{\pi \tau}
$$

que, usando $h^{\mu \nu}=\eta^{\mu \nu}+\kappa \phi^{\mu \nu}$, é equivalente ao shift da Eq. (5.23).

\subsubsection{Condição de consistência}

Enquanto as funções de Green no formalismo diagonal são geradas por $Z_{\mathrm{EH}}^{\mathrm{Id}}$ dado na Eq. (4.30), alternativamente podemos considerar o funcional gerador na forma derivada na Eq. (5.26):

$$
\begin{aligned}
Z_{\mathrm{EH}}^{\mathrm{Id}}[j, J] & =\int \mathcal{D} d_{\nu} \mathcal{D} \bar{d}_{\mu} \mathcal{D} \phi^{\mu \nu} \exp i\left[S_{\mathrm{eff}}^{\mathrm{II}}+\int d^{d} x j_{\mu \nu} \phi^{\mu \nu}\right. \\
& \left.+\int d^{d} x\left(J_{\lambda}^{\mu \nu} \Delta_{\mu \nu}^{\lambda}-\frac{\kappa^{2}}{2} J_{\lambda}^{\mu \nu}\left(M^{-1}\right)_{\mu \nu \pi \tau}^{\lambda \rho}(\eta+\kappa \phi) J_{\rho}^{\pi \tau}\right)\right] .
\end{aligned}
$$

onde $\Delta_{\mu \nu}^{\lambda}$ é o campo composto definido na Eq. (5.29) e a ação efetiva (clássica) $S_{\text {eff }}^{\mathrm{II}}$ do formalismo de segunda ordem é definida como

$$
S_{\mathrm{eff}}^{\mathrm{II}} \equiv \int d^{d} x \mathcal{L}_{\mathrm{eff}}^{\mathrm{II}} \cdot
$$


Entretanto, as funções de Green obtidas a partir do funcional gerador do lado direito da Eq. (5.34) são computadas no formalismo de segunda ordem da gravitação. Sendo assim, para o formalismo diagonal ser consistente, certas identidades entre funções de Green computadas no formalismo diagonal e no formalismo de segunda ordem devem ser satisfeitas. Uma dessas identidades já foi derivada, a Eq. (5.28), que demostra a equivalência das funções de Green de $n$-pontos do campo de calibre $\phi^{\mu \nu}$ nos dois formalismos.

Para funções de Green com campos auxiliares $H_{\mu \nu}^{\lambda}$ externos essas identidades são mais elaboradas. Para ver isso considere o caso da função de Green de um ponto que é gerada pela derivada funcional da Eq. (4.30) em relação a fonte $J_{\lambda}^{\mu \nu}(x) \operatorname{com} J=j=0$ :

$$
\left\langle 0\left|T H_{\mu \nu}^{\lambda}(x)\right| 0\right\rangle=-\left.i \frac{\delta Z_{\mathrm{EH}}^{\mathrm{I}}}{\delta J_{\lambda}^{\mu \nu}(x)}\right|_{j=J=0} .
$$

Enquanto a derivação do funcional gerador dado na sua alternativa (5.26) pela mesma fonte leva a correlações (na presença de fontes) do operador $\Delta_{\mu \nu}^{\lambda}(x)$ :

$$
\begin{aligned}
\frac{1}{i} \frac{\delta Z_{\mathrm{EH}}^{\mathrm{I}}[j, J]}{\delta J_{\lambda}^{\mu \nu}(x)} & =\frac{1}{i} \frac{\delta}{\delta J_{\lambda}^{\mu \nu}(x)} \int \mathcal{D} d_{\nu} \mathcal{D} \bar{d}_{\mu} \mathcal{D} \phi^{\mu \nu} \exp i\left[S_{\mathrm{eff}}^{\mathrm{II}}+\int d^{d} x j_{\mu \nu} \phi^{\mu \nu}\right. \\
& \left.+\int d^{d} x\left(J_{\lambda}^{\mu \nu} \Delta_{\mu \nu}^{\lambda}-\frac{\kappa^{2}}{2} J_{\lambda}^{\mu \nu}\left(M^{-1}\right)_{\mu \nu \pi \tau}^{\lambda \rho}[\eta+\kappa \phi(x)] J_{\rho}^{\pi \tau}\right)\right] . \\
& =\int \mathcal{D} d_{\nu} \mathcal{D} \bar{d}_{\mu} \mathcal{D} \phi^{\mu \nu}\left[\Delta_{\mu \nu}^{\lambda}(x)-\kappa^{2}\left(M^{-1}\right)_{\mu \nu \pi \tau}^{\lambda \rho}[\eta+\kappa \phi(x)] J_{\rho}^{\pi \tau}\right] \exp i S^{(\mathrm{I}, \mathrm{II})} \\
& \equiv\left\langle 0\left|T\left[\Delta_{\mu \nu}^{\lambda}(x)-\kappa^{2}\left(M^{-1}\right)_{\mu \nu \pi \tau}^{\lambda \rho}[\eta+\kappa \phi(x)] J_{\rho}^{\pi \tau}\right]\right| 0\right\rangle_{J},
\end{aligned}
$$

onde

$$
S^{(\mathrm{I}, \mathrm{II})} \equiv S_{\mathrm{eff}}^{\mathrm{II}}+\int d^{d} x\left(j_{\mu \nu} \phi^{\mu \nu}+J_{\lambda}^{\mu \nu} \Delta_{\mu \nu}^{\lambda}-\frac{\kappa^{2}}{2} J_{\lambda}^{\mu \nu}\left(M^{-1}\right)_{\mu \nu \pi \tau}^{\lambda \rho}[\eta+\kappa \phi(x)] J_{\rho}^{\pi \tau}\right)
$$

Assim como na Eq. (3.27), temos uma dependência não usual com a fonte, por essa razão ao considerar as correlações do funcional gerador na forma alternativa dada na Eq. (5.26) é necessário tomar cuidado com termos devido a fonte $J$.

No limite em que as fontes se anulam, em especial $J=0$, a correlação acima passa a ser igual ao valor esperado no vácuo do campo composto $\Delta_{\mu \nu}^{\lambda}$ :

$$
\left\langle 0\left|T\left[\Delta_{\mu \nu}^{\lambda}(x)-\kappa^{2}\left(M^{-1}\right)_{\mu \nu \pi \tau}^{\lambda \rho}[\eta+\kappa \phi(x)] J_{\rho}^{\pi \tau}(x)\right]\right| 0\right\rangle_{J=0}=\left\langle 0\left|T \Delta_{\mu \nu}^{\lambda}(x)\right| 0\right\rangle .
$$

Pela sua definição na Eq. (5.29) vemos que o seu valor esperado no vácuo é dado por funções de Green computadas no formalismo de segunda ordem. Porém, além das divergências usuais encontradas no cálculo das funções de Green também temos divergências que surgem por efeito de curta distância já que $\Delta_{\mu \nu}^{\lambda}$ é um campo composto. Por isso a computação de correlações como a da Eq. (5.39) é mais complicada do que a das usuais funções de Green.

Com $J=j=0$, a Eq. (5.37) é igual a Eq. (5.36) e obtemos a identidade estrutural análoga a identidade (3.31):

$$
\left\langle 0\left|T H_{\mu \nu}^{\lambda}(x)\right| 0\right\rangle=\left\langle 0\left|T \Delta_{\mu \nu}^{\lambda}(x)\right| 0\right\rangle,
$$

onde o lado esquerdo é computado no formalismo diagonal da gravitação, enquanto o lado direito é computado na formulação de segunda ordem. 
Esse é mais um exemplo de identidade estrutural consequente da condição de consistência para os funcionais geradores (4.30) e (5.26) do formalismo diagonal. Sendo que identidade (5.28) foi a primeira identidade derivada para garantir a consistência do formalismo diagonal, além de ser a mais relevante já que fisicamente ela garante a equivalência entre os formalismos.

Pela Eq. (3.15) também podemos computar o lado esquerdo da Eq. (5.40) no formalismo diagonal. Assim elevamos a identidade acima para

$$
\left\langle 0\left|T H_{\mu \nu}^{\lambda}(x)\right| 0\right\rangle=\left\langle 0\left|T \Delta_{\mu \nu}^{\lambda}(x)\right| 0\right\rangle \quad \text { (no formalismo de primeira ordem) }
$$

Essa identidade pode ser vista como uma generalização quântica da equação de movimento do campo auxiliar $H_{\mu \nu}^{\lambda}$ que é dada pela Eq. (2.22).

A Eq. (5.40) é exemplo simples de uma das identidades que podem ser obtida como consequência da Eq. (5.26) pela equivalência dos formalismos de primeira e segunda ordem da gravitação. A seguir deduziremos outras identidades estruturais semelhantes que são análogas às obtidas no capítulo 3. Tais identidades relacionam as funções de Green do formalismo diagonal e de segunda ordem da gravitação.

\subsubsection{Identidades estruturais}

Nessa seção deduziremos identidades estruturais mais elaboradas. Para isso iremos considerar derivadas funcionais do funcional gerador no formalismo diagonal tanto na forma usual (4.30) quanto para a forma alternativa (5.26). As identidades estruturais são derivadas quando as fontes se anulam, $j=J=0$, obtendo uma igualdade entre correlações computadas em diferentes formalismos.

\section{FunÇÕES DE GReEn De 2-POntos}

Derivando a Eq. (4.30) em relação as fontes $J_{\lambda}^{\mu \nu}(x)$ e $j_{\pi \tau}(y)$ obtemos a função de Green mista com um campo auxiliar $H_{\mu \nu}^{\lambda}(x)$ e um campo de calibre $\phi^{\pi \tau}(y)$ externo:

$$
\left.(-i)^{2} \frac{\delta^{2} Z_{\mathrm{EH}}^{\mathrm{Id}}[j, J]}{\delta J_{\lambda}^{\mu \nu}(x) \delta j_{\pi \tau}(y)}\right|_{j=J=0}=\left\langle 0\left|T H_{\mu \nu}^{\lambda}(x) \phi^{\pi \tau}(y)\right| 0\right\rangle .
$$

Enquanto para a forma alternativa na Eq. (5.26) obtemos

$$
\left\langle 0\left|T\left[\Delta_{\mu \nu}^{\lambda}(x)-\kappa^{2}\left(M^{-1}\right)_{\mu \nu \alpha \beta}^{\lambda \gamma}[\eta+\kappa \phi(x)] J_{\gamma}^{\alpha \beta}\right] \phi^{\pi \tau}(y)\right| 0\right\rangle_{J},
$$

que é similar a Eq. (3.43), mostrando, como vimos anteriormente, que a derivada funcional em relação a $J$ leva a uma correlação com uma dependência na mesma.

Com $j=J=0$ podemos igualar as Eqs. (5.42) e (5.43), tal que, [16]

$$
\left\langle 0\left|T H_{\mu \nu}^{\lambda}(x) \phi^{\pi \tau}(y)\right| 0\right\rangle=\left\langle 0\left|T \Delta_{\mu \nu}^{\lambda}(x) \phi^{\pi \tau}(y)\right| 0\right\rangle
$$

com o campo composto $\Delta_{\mu \nu}^{\lambda}$ definido pela Eq. (5.29). A identidade estrutural acima relaciona o propagador completo misto do formalismo diagonal $\left\langle 0\left|T H_{\mu \nu}^{\lambda}(x) \phi^{\pi \tau}(y)\right| 0\right\rangle$ com a correlação $\left\langle 0\left|T \Delta_{\mu \nu}^{\lambda}(x) \phi^{\pi \tau}(y)\right| 0\right\rangle$ computada, a princípio, no formalismo de segunda ordem. 
Para finalizar resta a função de Green de 2-pontos para o campo auxiliar $H_{\mu \nu}^{\lambda}$. A segunda derivada da Eq. (4.30) em relação a fonte $J$ leva a uma função de Green como

$$
\left\langle 0\left|T H_{\mu \nu}^{\lambda}(x) H_{\pi \tau}^{\rho}(y)\right| 0\right\rangle .
$$

Enquanto tomando a derivada funcional em relação a $J_{\rho}^{\pi \tau}(y)$ na Eq. (5.37), temos que

$$
\begin{aligned}
& \left\langle 0\left|T H_{\mu \nu}^{\lambda}(x) H_{\pi \tau}^{\rho}(y)\right| 0\right\rangle_{J} \\
& =\frac{1}{i} \frac{\delta}{\delta J_{\rho}^{\pi \tau}(y)} \int \mathcal{D} d_{\nu} \mathcal{D} \bar{d}_{\mu} \mathcal{D} \phi^{\mu \nu}\left[\Delta_{\mu \nu}^{\lambda}(x)-\kappa^{2}\left(M^{-1}\right)_{\mu \nu \alpha \beta}^{\lambda \gamma}[\eta+\kappa \phi(x)] J_{\gamma}^{\alpha \beta}\right] \exp i S^{(\mathrm{I}, \mathrm{II})} \\
& =\left\langle 0\left|T\left[\Delta_{\mu \nu}^{\lambda}(x)-\kappa^{2}\left(M^{-1}\right)_{\mu \nu \alpha^{\prime} \beta^{\prime}}^{\lambda} J_{\gamma^{\prime}}^{\alpha^{\prime} \beta^{\prime}}(x)\right]\left[\Delta_{\pi \tau}^{\rho}(y)-\kappa^{2}\left(M^{-1}\right)_{\pi \tau \alpha \beta}^{\rho \gamma}[\eta+\kappa \phi(x)] J_{\gamma}^{\alpha \beta}(y)\right]\right| 0\right\rangle_{J} \\
& -\frac{1}{i} \kappa^{2} \delta(x-y)\left\langle 0\left|T\left(M^{-1}\right)_{\mu \nu \pi \tau}^{\lambda \rho}[\eta+\kappa \phi(x)]\right| 0\right\rangle_{J} .
\end{aligned}
$$

Com $j=J=0$ na Eq. (5.46) deduzimos a identidade estrutural [16]

$$
\left\langle 0\left|T H_{\mu \nu}^{\lambda}(x) H_{\pi \tau}^{\rho}(y)\right| 0\right\rangle=\left\langle 0\left|T \Delta_{\mu \nu}^{\lambda}(x) \Delta_{\pi \tau}^{\rho}(y)\right| 0\right\rangle+i \kappa^{2} \delta(x-y)\left\langle 0\left|T\left(M^{-1}\right)_{\mu \nu \pi \tau}^{\lambda \rho}[\eta+\kappa \phi(x)]\right| 0\right\rangle .
$$

Essa identidade relaciona o propagador completo $\left\langle 0\left|T H_{\mu \nu}^{\lambda}(x) H_{\pi \tau}^{\rho}(y)\right| 0\right\rangle$ do formalismo diagonal da gravitação com a correlação $\left\langle 0\left|T \Delta_{\mu \nu}^{\lambda}(x) \Delta_{\pi \tau}^{\rho}(y)\right| 0\right\rangle$ calculada no formalismo de segunda ordem da gravitação. Assim como na Eq. (3.44), análoga a Eq. (5.47), temos um termo de contato que surge pelas derivadas sucessivas em relação a $J$.

\section{FunÇÕES DE GREen DE $(n+1)$-PONTOS}

Já a generalização da Eq. (5.44) é imediata; assim como generalizamos a Eq. (3.35) pela Eq. (3.48) podemos generalizar a Eq. (5.44) como [15]

$$
\left\langle 0\left|T H_{\mu \nu}^{\lambda}(x) \phi^{\pi_{1} \tau_{1}}\left(y_{1}\right) \cdots \phi^{\pi_{n} \tau_{n}}\left(y_{n}\right)\right| 0\right\rangle=\left\langle 0\left|T \Delta_{\mu \nu}^{\lambda}(x) \phi^{\pi_{1} \tau_{1}}\left(y_{1}\right) \cdots \phi^{\pi_{n} \tau_{n}}\left(y_{n}\right)\right| 0\right\rangle .
$$

De certa forma essa identidade pode ser interpretada como a realização quântica da equação de movimento clássica do campo auxiliar $H_{\mu \nu}^{\lambda}$ (ver Eq. (4.36)), assim como a Eq. (3.48) para a equação de movimento (2.16) do campo auxiliar $F_{\mu \nu}^{a}$.

No próximo capítulo vamos verificar explicitamente, no espaço do momento, as identidades (5.44) e (5.47) que valem em qualquer ordem, para qualquer dimensão $d>2$ e calibre $\alpha$ até um loop. Será necessário considerar as divergências que surgem devido a efeitos de curta distância pela presença do campo composto $\Delta_{\mu \nu}^{\lambda}$ que são essenciais para o cancelamento das divergências ultravioleta.

\subsection{Equivalência geral}

Pelas Eqs. (5.17) e (5.27), temos que

$$
Z_{\mathrm{EH}}^{\mathrm{II}}[j]=Z_{\mathrm{EH}}^{\mathrm{I}}[j, 0]=Z_{\mathrm{EH}}^{\mathrm{Id}}[j, 0]
$$


mostrando que todos os formalismos considerados para gravitação são equivalentes no nível quântico; isso é, as funções de Green com campos de calibre $\phi^{\mu \nu}$ externos são iguais em todos os formalismos:

$$
\begin{aligned}
\left.\frac{\delta^{n} Z_{\mathrm{EH}}^{\mathrm{II}}[j]}{\delta j_{\mu_{1} \nu_{1}}\left(x_{1}\right) \cdots \delta j_{\mu_{n} \nu_{n}}\left(x_{n}\right)}\right|_{j=0} & =\left.\frac{\delta^{n} Z_{\mathrm{EH}}^{\mathrm{I}}[j, J]}{\delta j_{\mu_{1} \nu_{1}}\left(x_{1}\right) \cdots \delta j_{\mu_{n} \nu_{n}}\left(x_{n}\right)}\right|_{J=j=0} \\
& =\left.\frac{\delta^{n} Z_{\mathrm{EH}}^{\mathrm{Id}}[j, J]}{\delta j_{\mu_{1} \nu_{1}}\left(x_{1}\right) \cdots \delta j_{\mu_{n} \nu_{n}}\left(x_{n}\right)}\right|_{J=j=0}
\end{aligned}
$$

Identidades análogas as Eqs. (3.68) e (3.69) também podem ser derivadas na gravitação. Por exemplo:

$$
\begin{aligned}
& \left\langle 0\left|T G_{\mu \nu}^{\lambda}(x) \phi^{\alpha \beta}(y)\right| 0\right\rangle-\left\langle 0\left|T H_{\mu \nu}^{\lambda}(x) \phi^{\alpha \beta}(y)\right| 0\right\rangle \\
& =\kappa\left\langle 0\left|T\left(M^{-1}\right)_{\mu \nu \pi \tau}^{\lambda \rho}[\eta+\kappa \phi(x)] \phi_{, \rho}^{\pi \tau}(x) \phi^{\alpha \beta}(y)\right| 0\right\rangle-\left\langle 0\left|T \Delta_{\mu \nu}^{\lambda}(x) \phi^{\alpha \beta}(y)\right| 0\right\rangle \\
& =\left\langle 0\left|T\left(M^{-1}\right)_{\mu \nu \pi \tau}^{\lambda \rho}(\eta) h_{, \rho}^{\pi \tau}(x) \phi^{\alpha \beta}(y)\right| 0\right\rangle
\end{aligned}
$$

que pode ser vista como a realização quântica da relação clássica entre $G_{\mu \nu}^{\lambda}$ e $H_{\mu \nu}^{\lambda}$ dada na Eq. (5.31); mostrando que podemos obter relações equivalentes as identidades estruturais derivadas pela comparação dos funcionais geradores da formulação de primeira ordem e a formulação diagonal da gravitação. Embora sejam equivalentes, tais relações não são iguais. Essas relações são mais restritas já que elas decorrem da equivalência geral entre todos os formalismos, conforme a Eq. (5.50).

Podemos estender a Eq. (5.49) para $J \neq 0$, como fizemos para a teoria de YM, definindo funcionais geradores generalizados:

$$
Z_{\mathrm{EH}}^{\mathrm{I}}[j, J]=\tilde{Z}_{\mathrm{EH}}^{\mathrm{II}}[j, J]=\tilde{Z}_{\mathrm{EH}}^{\mathrm{Id}}[j, J]
$$

onde

$$
\begin{aligned}
\tilde{Z}_{\mathrm{EH}}^{(\mathrm{Id})}[j, J] & =\int \mathcal{D} d_{\nu} \mathcal{D} \bar{d}_{\mu} \mathcal{D} H_{\mu \nu}^{\lambda} \mathcal{D} \phi^{\mu \nu} \\
& \quad \exp i \int d^{d} x\left(\mathcal{L}_{\mathrm{eff}}^{\mathrm{Id}}+j_{\mu \nu} \phi^{\mu \nu}+J_{\lambda}^{\mu \nu} H_{\mu \nu}^{\lambda}+J_{\lambda}^{\mu \nu}\left(M^{-1}\right)_{\mu \nu \pi \tau}^{\lambda \rho}(\eta) h_{, \rho}^{\pi \tau}\right) .
\end{aligned}
$$

e $\tilde{Z}_{\mathrm{EH}}^{\mathrm{II}}[j, J]$ é igual a forma alternativa do funcional gerador do formalismo de primeira ordem dado na Eq. (5.16). Quando temos $J=0$ é claro que a Eq. (5.52) se reduz a Eq. (5.49).

Os funcionais geradores da Eq. (5.53) e (5.16) são generalizações para os funcionais geradores $Z_{\mathrm{EH}}^{\mathrm{Id}}[j, 0]$ e $Z_{\mathrm{EH}}^{\mathrm{II}}[j]$ derivadas do funcional gerador do formalismo de primeira ordem $Z_{\mathrm{EH}}^{\mathrm{I}}$. Vale mencionar que o funcional gerador generalizado do formalismo diagonal $\tilde{Z}_{\mathrm{EH}}^{\mathrm{Id}}$ é obtido através do shift (nada mais que a relação clássica (5.31))

$$
G_{\mu \nu}^{\lambda}=H_{\mu \nu}^{\lambda}+\left(M^{-1}\right)_{\mu \nu \pi \tau}^{\lambda \rho}(\eta) h_{, \rho}^{\pi \tau}
$$

no funcional gerador $Z_{\mathrm{EH}}^{\mathrm{I}}[j, J]$. Também podemos obter generalizações a partir do formalismo diagonal, por exemplo, o funcional gerador na Eq. (5.34) é uma generalização do funcional gerador do formalismo de segunda ordem derivado do formalismo diagonal.

A abordagem acima permite que vejamos a equivalência de uma forma mais ampla. Em que todos os formalismos são equivalentes de forma geral, onde não só consideramos os graus de 
liberdades físicos do campo $\phi^{\mu \nu}$, mas também do campo auxiliar seja $G_{\mu \nu}^{\lambda}$ ou $H_{\mu \nu}^{\lambda}$. De forma genérica, podemos afirmar ${ }^{7}$ que

$$
\begin{aligned}
\text { Formalismo de Segunda Ordem } & \equiv \text { Formalismo de Primeira Ordem } \\
& \equiv \text { Formalismo Diagonal }
\end{aligned}
$$

\footnotetext{
${ }^{7}$ Lembrando que estamos considerando o caso da gravitação pura, sem campos de matéria.
} 


\section{VerificaÇÃo Explícita das IDEntidades \\ ESTRUTURAIS}

Iremos verificar explicitamente algumas das identidades estruturais derivadas nos capítulos 3 e 5 em até um loop. Para o computação de diagramas com divergências UV (um ou mais loops) é necessário considerar um método de regularização; o método que usaremos é de regularização dimensional ${ }^{1}$ como já mencionamos no capítulo anterior.

Para verificar tais identidades mostraremos como computar diagramas "pinched" [15, 16]. Tais diagramas, devido a campos compostos, são iguais a diagramas usuais onde certas pernas externas convergem ao mesmo ponto do espaço-tempo.

Para a computação das integrais dos diagramas com um loop implementamos o procedimento detalhado no apêndice D usando um software de computação simbólica obtendo os resultados ${ }^{2}$ publicados nos artigos $[15,16]$ onde verificamos as identidades (3.35), (3.44), (3.64b), (3.64c), (5.44) e (5.47).

\subsection{Teoria de Yang-Mills}

Nessa seção verificaremos as identidades (3.35) e (3.44) no nível árvore e a identidade (3.31) no nível de um loop (menor ordem). Já as identidades obtidas para o formalismo diagonal, (3.64b) e (3.64c), até um loop. Nesse último caso, mostraremos que a verificação vale até no nível de integrando.

Primeiramente vamos computar o lado esquerdo dessas identidades dado por uma função de Green usual do formalismo diagonal (ou de primeira ordem). Depois, o lado direito que é igual a

\footnotetext{
${ }^{1}$ Além de ser mais apropriado para o caso de teorias de calibre, como mencionamos no apêndice D; na seção anterior foi discutido que nesse método o determinante da Eq. (5.7) é reduzido a uma constante, isso permite que possamos integrar completamente o campo auxiliar e assim demonstrar a equivalência entre os formalismos no caso da gravitação.

${ }^{2} \mathrm{Na}$ gravitação, temos uma diferença de $\kappa^{2}$ devido a um pequeno erro no artigo [16] que considerou as regras para $\partial \cdot h$ invés de $\partial \cdot \phi$.
} 
uma correlação contendo o campo composto $h_{\mu \nu}^{a}\left(f_{\mu \nu}^{a}\right)$ computado no formalismo de segunda ordem. Para obter tais correlações será necessário uma análise das divergências devidas a esses campos mostrando que os diagramas "pinched" são iguais a diagramas com loops no espaço de momento.

\subsubsection{Verificação da identidade (3.31)}

É de amplo conhecimento que a função de Green de 1-ponto de qualquer campo (relativístico e quântico) é igual a zero (exceto com quebra espontânea de simetria [22]); caso contrário é possível realizar uma redefinição no campo tal que a função de Green de 1-ponto se anule. Evidentemente isso também vale para os campos $A_{\mu}^{a}$ e $F_{\mu \nu}^{a}$, isso é,

$$
\begin{gathered}
\left\langle 0\left|T A_{\mu}^{a}(x)\right| 0\right\rangle=0, \\
\left\langle 0\left|T F_{\mu \nu}^{a}(x)\right| 0\right\rangle=0 .
\end{gathered}
$$

Por vias das dúvidas consideremos os diagramas na Fig. 6.1 que contribuem perturbativamente para a função de 1-ponto dos campos considerados. Esses diagramas são do tipo "tadpole"3, ou seja, diagramas com uma perna externa, que nas teorias consideradas (teorias de calibre não massivas) se anulam usando regularização dimensional [38].

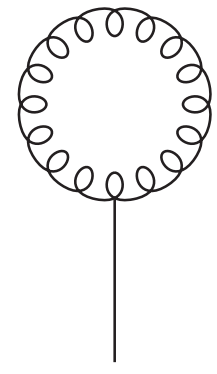

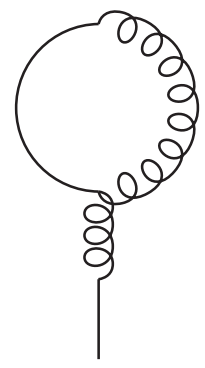

(a)
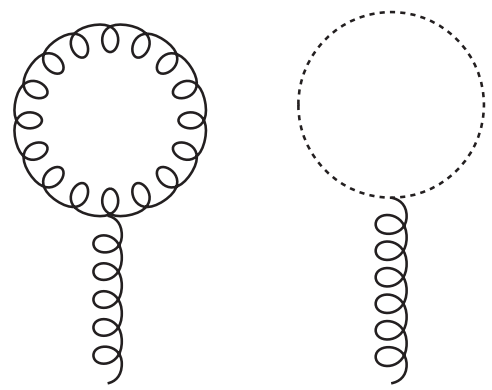

(b)

Figura 6.1: Diagramas tadpoles (a) de $\left\langle 0\left|T F_{\mu \nu}^{a}\right| 0\right\rangle$. Tadpoles (b) de $\left\langle 0\left|T A_{\mu}^{a}\right| 0\right\rangle$.

No caso da teoria de YM esses diagramas devem ser nulos em qualquer ordem, pois a contração do propagador com um vértice leva a um fator de cor igual a $f^{a b c} \delta^{b c}=f^{a b b}$ que é identicamente nulo (pela assimetria de $f^{a b c}$ ). No entanto, isso não vale para o caso da gravitação então seguimos exemplificando a computação de um desses diagramas mostrando que o mesmo deve ser anular usando regularização dimensional.

Por exemplo, o diagrama do campo fantasma na Fig. 6.1(b), pelas regras de Feynman (2.13), é proporcional a integral

$$
\int \frac{d^{d} p}{(2 \pi)^{d}} \frac{k^{\mu}}{p^{2}}
$$

\footnotetext{
${ }^{3}$ A tradução é "girino"; o termo surgiu pela a forma semelhante desses diagramas com girinos (fase de larva de anfíbios).
} 

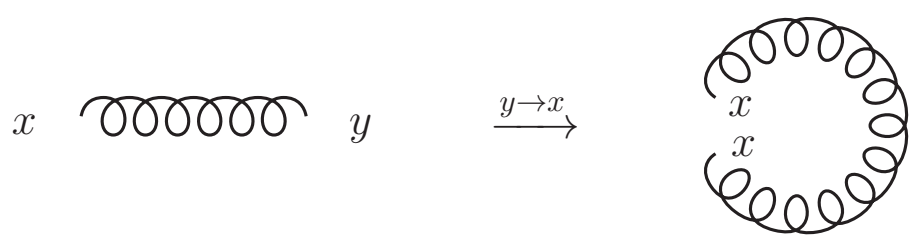

Figura 6.2: Diagrama tipo "pinched" que contribui na ordem $g$ de $\left\langle 0\left|T h_{\mu \nu}^{a}(x)\right| 0\right\rangle$ obtido através do propagador do campo de calibre $\left\langle A_{\mu}^{b}(x) A_{\nu}^{c}(y)\right\rangle$.

onde $k^{\mu}$ é o momento da perna externa ${ }^{4}$. Já a integral (6.2) é igual a $I_{2}[d] \operatorname{com} \Delta=0$; usando $\Delta=0$ na Eq. (D.10) obtemos que $I_{2}[d]=0$ por regularização dimensional, logo confirma-se que a integral (6.2) é nula (usando regularização dimensional).

Pela Eq. (6.1b) a identidade (3.31) é equivalente a

$$
\left\langle 0\left|T f_{\mu \nu}^{a}(x)\right| 0\right\rangle=0
$$

porém pela definição do campo composto, temos que

$$
\left\langle 0\left|T f_{\mu \nu}^{a}(x)\right| 0\right\rangle=\left\langle 0\left|T \partial_{\mu} A_{\nu}^{a}(x)-\partial_{\nu} A_{\mu}^{a}(x)+h_{\mu \nu}^{a}(x)\right| 0\right\rangle,
$$

onde $h_{\mu \nu}^{a}(x)$ é o campo composto definido na Eq. (3.62). A Eq. (6.4) pode por sua vez ser simplificada usando a Eq. (6.1a), tal que a identidade (3.31) se reduz a

$$
\left\langle 0\left|T h_{\mu \nu}^{a}(x)\right| 0\right\rangle=g f^{a b c}\left\langle 0\left|T A_{\mu}^{b}(x) A_{\nu}^{c}(x)\right| 0\right\rangle=0 .
$$

A identidade (6.5) mostra que a correlação do campo composto $h_{\mu \nu}^{a}(x)$ deve ser nula em qualquer ordem. Isso pode ser visto,em qualquer ordem, notando que todas as contribuições diagramáticas para o propagador $\left\langle 0\left|T A_{\mu}^{b}(x) A_{\nu}^{c}(y)\right| 0\right\rangle$ no limite em que $y \rightarrow x$ levam a tadpoles. Ou mais diretamente, no caso da teria de YM, notando que o propagador da Eq. (6.5) é proporcional a $\delta^{b c}$, tal que, $\left\langle 0\left|T h_{\mu \nu}^{a}(x)\right| 0\right\rangle \sim f^{a b c} \delta^{b c}=f^{a b b}=0$.

Para a contribuição de menor ordem temos o diagrama da Fig. 6.2. Esse diagrama é do tipo "pinched" $[15]$ onde as pernas externas estão no mesmo ponto do espaço-tempo $x$. Note que o diagrama pinched formado pela contração das pernas externas é similar ao gráfico de um loop. Demostraremos a seguir que tal semelhança é válida já que no espaço de momento esses diagramas levam a integrais divergente semelhantes as integrais devido a diagramas com loops.

Como vemos na Fig. 6.2 obtemos o diagrama pinched pela contração das pernas externa do propagador livre do campo de calibre. Seja o propagador inteiro $\left\langle 0\left|T A_{\mu}^{b}(x) A_{\nu}^{c}(y)\right| 0\right\rangle$ dado no espaço de momento por (ver Eq. (C.16))

$$
\left\langle 0\left|T A_{\mu}^{b}(x) A_{\nu}^{c}(y)\right| 0\right\rangle=\int \frac{d^{d} p}{(2 \pi)^{d}}\left\langle 0\left|T A_{\mu}^{b}(-p) A_{\nu}^{c}(p)\right| 0\right\rangle e^{-i p(y-x)},
$$

\footnotetext{
${ }^{4}$ Pela conservação de momento esse momento deve ser nulo, porém para ilustrar consideraremos o cálculo da integral antes de usar o delta de conservação

"A tradução literal seria "belisco". Como no caso dos diagramas "girinos", os diagramas "beliscos" têm esse nome pela a semelhança dos gráficos com o ato de beliscar.
} 


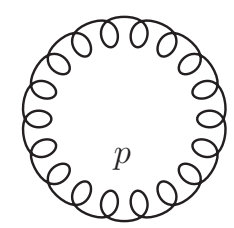

(a)

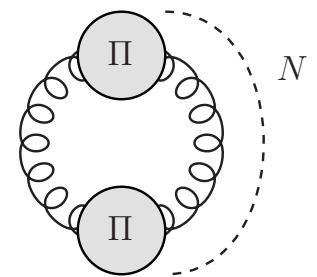

(b)

Figura 6.3: A bolha de vácuo (a) é igual ao diagrama pinched da Fig. 6.2 no espaço do momento. O diagrama (b) é um exemplo de diagrama anelar de $N+1$ loops, onde $N$ é o número de autoenergias inseridas, que contribui para $\left\langle 0\left|T A_{\mu}^{b}(x) A_{\nu}^{c}(x)\right| 0\right\rangle$.

o resultado, em qualquer ordem, para o lado esquerdo da Eq. (6.5) no espaço de momento é igual a Eq. (6.6) no limite em que $y \rightarrow x$, tal que,

$$
\left\langle 0\left|T A_{\mu}^{b}(x) A_{\nu}^{c}(x)\right| 0\right\rangle=\int \frac{d^{d} p}{(2 \pi)^{d}}\left\langle 0\left|T A_{\mu}^{b}(-p) A_{\nu}^{c}(p)\right| 0\right\rangle .
$$

Pela expansão em loop que o propagador deve satisfazer (vide Eq. (B.27)) temos que a integral acima é igual a uma soma de diagramas anelares (na Fig. 6.3(b) é dado um exemplo de diagrama anelar com $N+1$ loops $^{6}$ ) intercalando o propagador e a autoenergia do campo de calibre ${ }^{7}$.

O diagrama pinched na Fig. 6.2 é o diagrama anelar de menor ordem que pela Eq. (6.7) deve ser igual a

$$
\left\langle A_{\mu}^{b}(x) A_{\nu}^{c}(x)\right\rangle=\int \frac{d^{d} p}{(2 \pi)^{d}} P_{\mu \nu}^{b c}(p)
$$

onde $P_{\mu \nu}^{b c}$ é o propagador do campo de calibre no espaço de momento definido na Eq. (C.17).

A integral (6.8) é igual a contribuição do diagrama da Fig. 6.3(a) demonstrando que no espaço do momento a contração das pernas externas leva numa integral de um diagrama com loop. Pela forma do propagador $P_{\mu \nu}^{b c}(p)$ é claro que a integral do lado direito da Eq. (6.8) é nula. Por exemplo, com $\xi=1$, a integral da Eq. (6.8) é proporcional a integral (6.2) que é nula. Portanto, verificamos que a Eq. (6.5) é válida até a ordem de um loop (ordem g). É evidente que o mesmo vale para a identidade (3.31).

Essa demostração mostra claramente como podemos obter os diagramas pinched diagramaticamente. A derivação direta das contribuições é um pouco mais complicada, porém mostraremos em detalhes no caso da teoria de YM para esclarecer como deve-se proceder para chegar nas divergências devido aos campos compostos.

\subsubsection{Verificação das identidades (3.35) e (3.44)}

A verificação das identidades (3.35) e (3.44) no nível árvore é interessante já que elas mostram relações entre as regras de Feynman do formalismo de primeira ordem e de segunda ordem. Além

\footnotetext{
${ }^{6} \mathrm{O}$ diagrama da Fig. 6.3(a) corresponde ao caso em que $N=0$.

${ }^{7} \mathrm{Na}$ Fig. 6.3(b) é representada genericamente por $\Pi$.
} 
disso, podemos mostrar como tratar as derivadas que aparecem nas correlações no espaço de momento para obter resultados de acordo com as convenções usadas.

\section{VERIFICAÇÃO DA IDENTIDADE (3.35)}

O lado esquerdo da identidade (3.35) no nível árvore é igual ao propagador misto $\left\langle F_{\mu \nu}^{a}(x) A_{\rho}^{b}(y)\right\rangle$ que no espaço de momento, é igual (ver (C.41c))

$$
\frac{p_{\mu} \eta_{\nu \rho}-p_{\nu} \eta_{\mu \rho}}{p^{2}} \delta^{a b}
$$

onde $p$ é o momento ${ }^{8}$ do propagador.

O lado direito é igual a

$$
\left\langle 0\left|T f_{\mu \nu}^{a}(x) A_{\rho}^{b}(y)\right| 0\right\rangle=\left\langle 0\left|T\left(\partial_{\mu} A_{\nu}^{a}(x)-\partial_{\nu} A_{\mu}^{a}(x)+g f^{a b c} A_{\mu}^{b}(x) A_{\nu}^{g}(x)\right) A_{\rho}^{b}(y)\right| 0\right\rangle .
$$

Porém, o termo $g f^{a b c} A_{\mu} A_{\nu}$ é de ordem superior ${ }^{9}$ por isso podemos desconsiderá-lo, tal que, a Eq. (6.9) deve ser igual a

$$
\left\langle\left(\partial_{\mu} A_{\nu}^{a}(x)-\partial_{\nu} A_{\mu}^{a}(x)\right) A_{\rho}^{b}(y)\right\rangle
$$

no espaço de momento.

Conforme a Eq. (C.8), a Eq. (6.11) no espaço de momento é igual a

$$
\int \frac{d^{d} p}{(2 \pi)^{d}}\left\langle\left(\partial_{\mu} A_{\nu}^{a}(-p)-\partial_{\nu} A_{\mu}^{a}(-p)\right) A_{\rho}^{b}(p)\right\rangle e^{-i p(y-x)} .
$$

As derivadas atuando na exponencial na Eq. (6.12) resultam em fatores de ip, tal que, a equação acima é igual a

$$
\int \frac{d^{d} p}{(2 \pi)^{d}} i\left(p_{\mu}\left\langle A_{\nu}(-p) A_{\rho}^{b}(p)\right\rangle-p_{\nu}\left\langle A_{\mu}^{a}(-p) A_{\rho}^{b}(-p)\right\rangle\right) e^{-i p(y-x)}
$$

Pela Eq. (C.8) identificamos a forma da Eq. (6.11) no espaço de momento como

$$
i p_{\mu}\left\langle A_{\nu}(-p) A_{\rho}^{b}(p)\right\rangle-i p_{\nu}\left\langle A_{\mu}^{a}(-p) A_{\rho}^{b}(-p)\right\rangle
$$

Usando a Eq. (C.17) que define o propagador do campo de calibre, a equação acima pode ser reescrita como

$$
\begin{gathered}
i p_{\mu} P_{\nu \rho}^{a b}-i p_{\nu} P_{\mu \rho}^{a b}=\frac{p_{\mu}}{p^{2}}\left(\eta_{\nu \rho}-(1-\xi) \frac{p_{\nu} p_{\rho}}{p^{2}}\right) \delta^{a b}-\frac{p_{\nu}}{p^{2}}\left(\eta_{\mu \rho}-(1-\xi) \frac{p_{\mu} p_{\rho}}{p^{2}}\right) \delta^{a b} . \\
\frac{p_{\mu}}{p^{2}} \eta_{\nu \rho} \delta^{a b}-\frac{p_{\nu}}{p^{2}} \eta_{\mu \rho} \delta^{a b}=\frac{p_{\mu} \eta_{\nu \rho}-p_{\nu} \eta_{\mu \rho}}{p^{2}} \delta^{a b}
\end{gathered}
$$

o propagador misto $\left\langle F_{\mu \nu}^{a}(-p) A_{\rho}^{b}(p)\right\rangle$; igual ao lado esquerdo da Eq. (6.9), demonstrando que a identidade Eq. (3.35) é válida no nível árvore.

\section{VERIFICAÇÃO DA IDENTIDADE (3.44)}

${ }^{8}$ Pela Eq. (C.8), as derivadas no espaço dos momentos são definidas por $\partial_{x} \rightarrow i p$ e $\partial_{y} \rightarrow-i p$.

${ }^{9}$ Como veremos adiante esse termo é de ordem $g^{2}$. Mas, evidentemente as contribuições desse termo são de ordem igual ou superior a $g$ sendo suficiente para descartá-lo. 
O lado esquerdo da identidade (3.44) no nível árvore é igual ao propagador $\left\langle F_{\mu \nu}^{a}(x) F_{\alpha \beta}^{b}(y)\right\rangle$ que no espaço de momento, é igual (ver (C.41d))

$$
2 i\left(I_{\mu \nu \rho \sigma}+L_{\mu \nu \rho \sigma}\right) \delta^{a b}
$$

onde $p$ é o momento do propagador e os tensores $I, L$ definidos, respectivamente, nas Eqs. (3.40) e (C.40).

O lado direito, ao nível do árvore, é igual a

$$
2 i I_{\mu \nu \rho \sigma} \delta(x-y) \delta^{a b}+\left\langle 0\left|T\left(\partial_{\mu} A_{\nu}^{a}(x)-\partial_{\nu} A_{\mu}^{a}(x)\right)\left(\partial_{\rho} A_{\sigma}^{b}(y)-\partial_{\sigma} A_{\rho}^{b}(y)\right)\right| 0\right\rangle .
$$

Como vimos acima, no espaço de momento, $\partial_{x} \rightarrow i p$ e $\partial_{y} \rightarrow-i p$, tal que, usando que a transformada de Fourier da função delta é igual a 1, podemos escrever a Eq. (6.11) no espaço de momento como

$$
2 i I_{\mu \nu \rho \sigma} \delta^{a b}+i\left\langle\left(p_{\mu} A_{\nu}^{a}(-p)-p_{\nu} A_{\mu}^{a}(-p)\right)\left(p_{\rho} A_{\sigma}^{b}(p)-p_{\sigma} A_{\rho}^{b}(p)\right)\right\rangle .
$$

Imediatamente nota-se que o primeiro termo da equação acima e da Eq. (6.17) são iguais, então basta mostrar que os últimos termos também concordam.

Podemos escrever o último termo da Eq. (6.19) em termo do propagador do campo de calibre:

$$
p_{\mu} p_{\rho} P_{\nu \sigma}^{a b}+p_{\nu} p_{\sigma} P_{\mu \rho}^{a b}-p_{\mu} p_{\rho} P_{\nu \sigma}^{a b}-p_{\nu} p_{\rho} P_{\mu \sigma}^{a b}
$$

Assim como no caso acima os termos proporcionais a $1-\xi$ se anulam; o restante pode ser obtido pela permutação dos índices do termo resultante de $p_{\mu} p_{\rho} P_{\nu \sigma}^{a b}$ igual a $-i p_{\mu} p_{\rho} \eta_{\nu \sigma} \delta^{a b}$. Considerando todas permutações possíveis pela simetria da Eq. (6.19) chegamos em $2 i L_{\mu \nu \rho \sigma} \delta^{a b}$. Ou seja, a Eq. (6.19) é igual a Eq. (6.17); demonstrando explicitamente a identidade Eq. (3.44) no nível de árvore.

\subsubsection{Correções quânticas em um loop}

Nessa seção mostraremos os resultados obtidos usando o procedimento detalhado no apêndice D para a computação da contribuição de um loop dos propagadores do formalismo diagonal que aparecem nas identidades (3.64b) e Eq. (3.64c). Usaremos uma abordagem diferente dos artigos $[15,16]$ para a obtenção (direta) de diagramas pinched além de mostrar ao mesmo tempo que os resultados obtidos valem no nível de integrando.

\section{Autoenergia mista $H A$}

Considere o diagrama da Fig. 6.4 que é a única contribuição para o propagador misto no nível de um loop. A amputação de suas pernas externas leva a autoenergia ${ }^{10}$ de $H A$ que pode ser computada usando o procedimento da seção D.2.1.

Pelas regras de Feynman da seção 2.3.1, o diagrama da Fig. 6.4, com pernas externas amputadas, é igual a

$$
\Pi_{\mu^{\prime} \nu^{\prime} \rho^{\prime}}^{a^{\prime} b^{\prime}}(k)=\frac{1}{2} \int \frac{d^{d} p}{(2 \pi)^{d}} \mathcal{V}_{\mu^{\prime} \nu^{\prime} \alpha \beta}^{a^{\prime} c d} P_{c c^{\prime}}^{\alpha \pi}(p) P_{d d^{\prime}}^{\beta \tau}(q) V_{\pi \tau \rho^{\prime}}^{c^{\prime} d^{\prime} b^{\prime}}(-p, q,-k),
$$

\footnotetext{
${ }^{10} \mathrm{~A}$ autoenergia é definida de forma geral na Eq. (B.26); mais detalhes são dados no mesmo apêndice.
} 


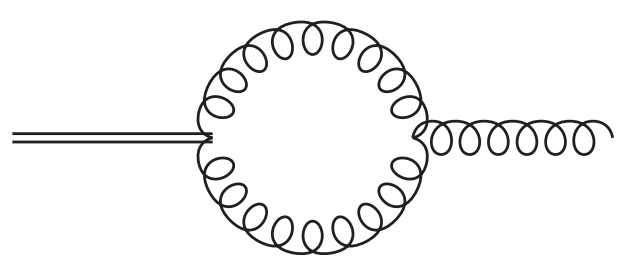

Figura 6.4: Diagrama que contribui para $\left\langle 0\left|T H_{\mu \nu}^{a} A_{\rho}^{b}\right| 0\right\rangle$ no nível de um loop (ordem $g^{2}$ ).

onde $1 / 2$ é o fator de simetria do diagrama; $\mathcal{V}_{\mu^{\prime} \nu^{\prime} \alpha^{\prime} \beta^{\prime}}^{a^{\prime} d d}$ é a regra para o vértice $(H A A)$ e $V_{\pi \tau \rho^{\prime}}^{c^{\prime} d^{\prime} b^{\prime}}(-p, q,-k)$ para o vértice $(A A A)$ definidas, respectivamente, nas Eqs. (C.42) e (C.23).

Considerando a simetria $\prod_{\mu^{\prime} \nu^{\prime} \rho^{\prime}}^{a^{\prime} b^{\prime}}(k)=-\Pi_{\nu^{\prime} \mu^{\prime} \rho^{\prime}}^{a^{\prime} b^{\prime}}(k)$ podemos decompor a integral da Eq. (6.21) como

$$
\Pi_{\mu^{\prime} \nu^{\prime} \rho^{\prime}}^{a^{\prime} b^{\prime}}(k)=c_{1} T_{\mu^{\prime} \nu^{\prime} \rho^{\prime}}(k) \delta^{a^{\prime} b^{\prime}}
$$

onde

$$
T_{\mu^{\prime} \nu^{\prime} \rho^{\prime}}=\frac{k_{\mu^{\prime}} \eta_{\nu^{\prime} \rho^{\prime}}-k_{\nu^{\prime}} \eta_{\mu^{\prime} \rho^{\prime}}}{p^{2}}
$$

que possui as mesmas simetrias que a autoenergia. Contraindo a Eq. (6.22) pelo único tensor da base (6.23) obtemos que

$$
\Pi_{\mu^{\prime} \nu^{\prime} \rho^{\prime}}^{a^{\prime} b^{\prime}} T^{\mu^{\prime} \nu^{\prime} \rho^{\prime}}(k)=2 \frac{d-1}{k^{2}} \delta^{a^{\prime} b^{\prime}} c_{1}
$$

tal que, obtemos $c_{1} \delta^{a^{\prime} b^{\prime}}$ através da integral escalar

$$
\frac{k^{2}}{4(d-1)} \int \frac{d^{d} p}{(2 \pi)^{d}} V_{\mu^{\prime} \nu^{\prime} \alpha \beta}^{a^{\prime} \alpha d} P_{c c^{\prime}}^{\alpha \pi}(p) P_{d d^{\prime}}^{\beta \tau}(q) V_{\pi \tau \rho^{\prime}}^{c^{\prime} d^{\prime} b^{\prime}}(-p, q,-k) T^{\mu^{\prime} \nu^{\prime} \rho^{\prime}}(k) .
$$

Computando ${ }^{11}$ a integral (6.25) obtemos a solução da Eq. (6.24):

$$
c_{1}=-i k^{2} g^{2} C_{\mathrm{YM}} I_{11} \frac{\left[(d-4) \xi^{2}+2(d-5) \xi-3 d+2\right]}{16},
$$

onde

$$
I_{11}=i^{1+d} \frac{\left(k^{2}\right)^{d / 2-2}}{(4 \pi)^{d / 2}} \frac{\Gamma(2-d / 2) \Gamma(d / 2-1)^{2}}{\Gamma(d-2)} ;
$$

uma integral básica calculada nos apêndices (ver Eq. (D.18)) e definimos $\delta^{a^{\prime} b^{\prime}} C_{\mathrm{YM}} \equiv f^{a^{\prime} l m} f^{b^{\prime} l m}$.

Substituindo a solução acima na Eq. (6.22) obtemos a autoenergia $\Pi_{\mu^{\prime} \nu^{\prime} \rho^{\prime}}^{a^{\prime} b^{\prime}}$ :

$$
-i k^{2} g^{2} I_{11} \delta^{a^{\prime} b^{\prime}} C_{\mathrm{YM}} \frac{\left[(d-4) \xi^{2}+2(d-5) \xi-3 d+2\right]}{16} \frac{k_{\mu^{\prime}} \eta_{\nu^{\prime} \rho^{\prime}}-k_{\nu^{\prime}} \eta_{\mu^{\prime} \rho^{\prime}}}{k^{2}} .
$$

Esse é o resultado geral para a autoenergia mista valendo para qualquer dimensão $d$ e qualquer calibre $\xi$. A divergência ultravioleta (UV) é obtida usando $d=4-2 \epsilon$ na Eq. (6.28), onde o polo UV é devido a $I_{11}$ que nesse limite é dado por $I_{\mathrm{UV}}$ definido na Eq. (D.22). Logo, o polo UV da autoenergia é igual a

$$
i g^{2} C_{\mathrm{YM}} I_{U V} \delta^{a^{\prime} b^{\prime}} \frac{\xi+5}{8}\left(k_{\mu^{\prime}} \eta_{\nu^{\prime} \rho^{\prime}}-k_{\nu^{\prime}} \eta_{\mu^{\prime} \rho^{\prime}}\right)=-\frac{g^{2} C_{\mathrm{YM}}}{128 \pi^{2} \epsilon}(\xi+5)\left(k_{\mu^{\prime}} \eta_{\nu^{\prime} \rho^{\prime}}-k_{\nu^{\prime}} \eta_{\mu^{\prime} \rho^{\prime}}\right) \delta^{a^{\prime} b^{\prime}} .
$$

\footnotetext{
${ }^{11}$ Usamos o software Mathematica para calcular as integrais e obter a solução do sistema de equações. Um exemplo de código usado é apresentado no final do apêndice D mostrando a verificação da identidade (3.64b) ao nível de um loop.
} 


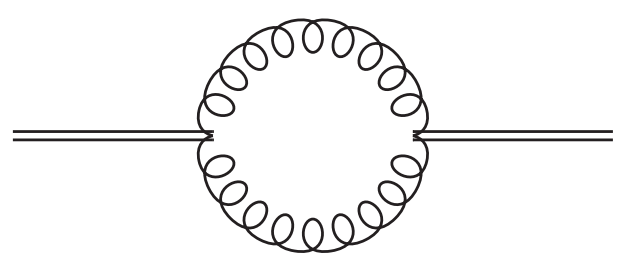

Figura 6.5: Diagrama que contribui para $\left\langle 0\left|T H_{\mu \nu}^{a} H_{\rho \sigma}^{b}\right| 0\right\rangle$ no nível de um loop (ordem $g^{2}$ ).

\section{Propagador $H A$}

Podemos obter a correção de um loop para o propagador $\left\langle H_{\mu \nu}^{a} A_{\rho}^{b}\right\rangle$, dado diagramaticamente na Fig. 6.4, a partir da contração da autoenergia $\Pi^{a b \mu^{\prime} \nu^{\prime} \rho^{\prime}}$ pelos propagadores do campo auxiliar $H$ e do campo de calibre $A$ :

$$
\left\langle H_{\mu \nu}^{a}(-k) H_{\mu^{\prime} \nu^{\prime}}^{a^{\prime}}(k)\right\rangle \Pi^{a b \mu^{\prime} \nu^{\prime} \rho^{\prime}}(k)\left\langle A_{\rho^{\prime}}^{b^{\prime}}(-k) A_{\rho}^{b}(k)\right\rangle .
$$

A contração dos propagadores não altera a estrutura tensorial da autoenergia resultando em um fator igual a $2 / k^{2} \delta^{a a^{\prime}} \delta^{b b^{\prime}}$. Sendo assim, a correção a nível de um loop para o propagador misto é dada simplesmente por

$$
-i g^{2} I_{11} \delta^{a b} C_{\mathrm{YM}} \frac{\left[(d-4) \xi^{2}+2(d-5) \xi-3 d+2\right]}{8} \frac{k_{\mu} \eta_{\nu \rho}-k_{\nu} \eta_{\mu \rho}}{k^{2}}
$$

cujo polo UV é igual a

$$
-\frac{g^{2} C_{\mathrm{YM}}}{64 \pi^{2} \epsilon}(5+\xi) \frac{k_{\mu} \eta_{\nu \rho}-k_{\nu} \eta_{\mu \rho}}{k^{2}} \delta^{a b},
$$

estando de acordo com [16, Eq. 2.13].

\section{Autoenergia Do CAMPo AUXiLiar $H$}

A autoenergia do campo auxiliar $H$ que é obtida pela amputação das pernas externas do diagrama da Fig. 6.5 (contribuição de um loop para o propagador $H$ ) pode ser obtida de forma similar a autoenergia mista calculada anteriormente ${ }^{12}$.

Pelas regras de Feynman na seção 2.3.1, o diagrama da Fig. 6.5, com pernas externas amputadas, é igual a

$$
\Pi_{\mu^{\prime} \nu^{\prime} \rho^{\prime} \sigma^{\prime}}^{a^{\prime} b^{\prime}}(k)=\frac{1}{2} \int \frac{d^{d} p}{(2 \pi)^{d}} \mathcal{V}_{\mu^{\prime} \nu^{\prime} \alpha \beta}^{a^{\prime} c d} P_{c c^{\prime}}^{\alpha \pi}(p) P_{d d^{\prime}}^{\beta \tau}(q) \mathcal{V}_{\pi \tau \rho^{\prime} \sigma^{\prime}}^{c^{\prime} d^{\prime} b^{\prime}}
$$

onde $1 / 2$ é o fator de simetria do diagrama.

Considerando as simetrias da autoenergia definida pela integral da Eq. (6.33) podemos usar os tensores $I$ e $L$ como base tensorial:

$$
\Pi_{\mu^{\prime} \nu^{\prime} \rho^{\prime} \sigma^{\prime}}^{a^{\prime} b^{\prime}}(k)=C_{1} I_{\mu^{\prime} \nu^{\prime} \rho^{\prime} \sigma^{\prime}}+C_{2} L_{\mu^{\prime} \nu^{\prime} \rho^{\prime} \sigma^{\prime}}
$$

\footnotetext{
${ }^{12}$ Poderíamos computar diretamente a correção do propagador já que o propagador do campo auxiliar é igual a identidade e não alteraria a base tensorial usada. Na verdade, isso é válido para todos casos considerados nesse capítulo. Na teoria de YM, temos que as correções de um loop para os propagadores têm a mesma estrutura tensorial do propagador livre, tal que, a base da autoenergia e do propagador coincidem. Já na gravitação, a base tensorial considerada é mais geral e vale para ambos.
} 
Contraindo a Eq. (6.34) pelo tensores da base obtemos o sistema linear de equações

$$
\begin{array}{r}
-8 d C_{1}^{\prime}+16 C_{2}^{\prime}-d(\xi-3)(\xi+1)+4 \xi^{2}-4=0, \\
16 C_{1}^{\prime}-16 C_{2}^{\prime}+(\xi+1)(d(\xi-1)-4 \xi)=0,
\end{array}
$$

onde $C_{i}=g^{2} C_{\mathrm{YM}} I_{11} \delta^{a^{\prime} b^{\prime}} C_{i}^{\prime}$, com $i=1,2$. Note que para obter o sistema acima já integramos as integrais escalares devido a contração de (6.35) com $I^{\mu^{\prime} \nu^{\prime} \rho^{\prime} \sigma^{\prime}}$ e $L^{\mu^{\prime} \nu^{\prime} \rho^{\prime} \sigma^{\prime}}$ usando as Eq. (D.19) e (D.20).

A solução do sistema Eq. (6.35) é igual a

$$
\left\{C_{1}^{\prime}=\frac{\xi+1}{4}, C_{2}^{\prime}=\frac{1}{16}(d-4)(\xi-1)(\xi+1)\right\},
$$

tal que, a autoenergia é dada por

$$
\Pi_{\mu^{\prime} \nu^{\prime} \rho^{\prime} \sigma^{\prime}}^{a^{\prime} b^{\prime}}(k)=g^{2} C_{\mathrm{YM}} I_{11} \delta^{a^{\prime} b^{\prime}}\left(\frac{\xi+1}{4} I_{\mu^{\prime} \nu^{\prime} \rho^{\prime} \sigma^{\prime}}+\frac{1}{16}(d-4)(\xi-1)(\xi+1) L_{\mu^{\prime} \nu^{\prime} \rho^{\prime} \sigma^{\prime}}\right) .
$$

Já o polo UV da autoenergia de $H$, obtido para $d=4-2 \epsilon$, é

$$
g^{2} C_{\mathrm{YM}} I_{\mathrm{UV}} \delta^{a^{\prime} b^{\prime}} \frac{\xi+1}{4} I_{\mu^{\prime} \nu^{\prime} \rho^{\prime} \sigma^{\prime}}=i \frac{g^{2} C_{\mathrm{YM}}}{64 \pi^{2} \epsilon}(\xi+1) I_{\mu^{\prime} \nu^{\prime} \rho^{\prime} \sigma^{\prime}} \delta^{a^{\prime} b^{\prime}}
$$

\section{PROPAGADOR $H_{\mu \nu}^{a} H_{\rho \sigma}^{b}$}

Podemos obter a correção de um loop para o propagador (dada pelo diagrama na Fig. 6.5) $\left\langle H_{\mu \nu}^{a} H_{\rho}^{b}\right\rangle$ a partir da contração da autoenergia $\Pi^{a b \mu^{\prime} \nu^{\prime} \rho^{\prime} \sigma}$ pelos propagadores do campo auxiliar $H$ :

$$
\left\langle H_{\mu \nu}^{a}(-k) H_{\mu^{\prime} \nu^{\prime}}^{a^{\prime}}(k)\right\rangle \Pi^{a^{\prime} b^{\prime} \mu^{\prime} \nu^{\prime} \rho^{\prime} \sigma^{\prime}}(k)\left\langle H_{\rho^{\prime} \sigma^{\prime}}^{b^{\prime}}(-k) H_{\rho \sigma}^{b}(k)\right\rangle .
$$

Pela definição do propagador do campo auxiliar $H_{\mu \nu}^{a}$ (C.47), o resultado da contração dos propagadores é igual a um fator $-4 \delta^{a a^{\prime}} \delta^{b^{\prime} b}$, tal que, a correção, a nível de um loop, para o propagador misto é dada por

$$
-g^{2} C_{\mathrm{YM}} I_{11} \delta^{a b}\left((\xi+1) I_{\mu \nu \rho \sigma}+\frac{1}{4}(d-4)(\xi-1)(\xi+1) L_{\mu \nu \rho \sigma}\right) .
$$

Nesse caso, o polo UV é mais simples:

$$
-i \frac{g^{2} C_{\mathrm{YM}}}{16 \pi^{2} \epsilon}(1+\xi) I_{\mu \nu \rho \sigma} \delta^{a b}
$$

e também está de acordo com o obtido em [16, Eq. 2.16].

\subsubsection{Diagramas pinched}

Nessa seção mostraremos os resultados obtidos usando o procedimento detalhado no apêndice D para a computação de diagramas pinched que contribuem para as correlações que aparecem no lado direito das Eqs. (3.64b) e (3.64c).

No nível de um loop só temos dois diagramas pinched revelantes: o diagrama da Fig. 6.6(a) que contribui para $g f^{a l m}\left\langle 0\left|T A_{\mu}^{l}(x) A_{\nu}^{m}(x) A_{\rho}^{b}(y)\right| 0\right\rangle$ e o diagrama da Fig. 6.6(b) que contribui para $g^{2} f^{a l m} f^{b l^{\prime} m^{\prime}}\left\langle 0\left|T A_{\mu}^{l}(x) A_{\nu}^{m}(x) A_{\rho}^{l^{\prime}}(y) A_{\sigma}^{m^{\prime}}(y)\right| 0\right\rangle$. 


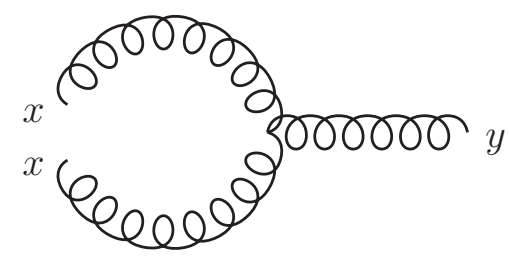

(a)

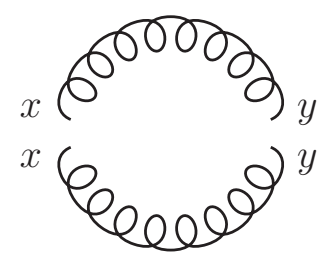

(b)

Figura 6.6: Diagramas pinched no nível de um loop (ordem $g^{2}$ ): (a) contribui para $g f^{a l m}\left\langle 0\left|T A_{\mu}^{l}(x) A_{\nu}^{m}(x) A_{\rho}^{b}(y)\right| 0\right\rangle$ e (b) contribui para $g^{2} f^{a l m} f^{b l^{\prime} m^{\prime}}\left\langle 0\left|T A_{\mu}^{l}(x) A_{\nu}^{m}(x) A_{\rho}^{l^{\prime}}(y) A_{\sigma}^{m^{\prime}}(y)\right| 0\right\rangle$.

Os diagramas pinched no espaço do momento são iguais a diagramas usuais do formalismo de segunda ordem, onde as contrações das pernas externas geram loops. No entanto, essa equivalência não é tão simples como no caso do diagrama da Fig. 6.2 que no espaço do momento é o diagrama anelar da Fig. 6.3(a). Por isso vamos considerar mais uma vez, em detalhes, a computação de um diagrama pinched. Em seguida mostraremos como pode-se obter tais diagramas diretamente usando uma extensão das regras de Feynman.

Considere o vértice $(A(x) A(y) A(z))$ (com propagadores externos) que dá origem ao diagrama da Fig. 6.6(a) quando $x=y$. No espaço de posições é dado por ${ }^{13}$

$$
(2 \pi)^{-2 d} \int d^{d} p d^{d} q d^{d} k \delta(-p+q-k) P_{\mu \alpha}^{l a^{\prime}}(p) P_{\nu \beta}^{m b^{\prime}}(q) P_{\rho \sigma}^{c c^{\prime}}(k) V^{a^{\prime} b^{\prime} c^{\prime} \alpha \beta \sigma}(-p, q,-k) e^{+i p x-i q y+i k z} .
$$

Para obter o diagrama pinched da Fig. 6.6(a) tomamos o limite em que $y \rightarrow x$ na integral acima obtendo:

$$
(2 \pi)^{-2 d} \int d^{d} p d^{d} q d^{d} k \delta(q-p-k) P_{\mu \alpha}^{l a^{\prime}}(p) P_{\nu \beta}^{m b^{\prime}}(q) P_{\rho \sigma}^{c c^{\prime}}(k) V^{a^{\prime} b^{\prime} c^{\prime} \alpha \beta \sigma}(-p, q,-k) e^{-i(q-p) x+i k z} .
$$

Ao integrar sobre $q$ usando a função $\delta(p+q-k)$ obtemos

$$
(2 \pi)^{-2 d} \int d^{d} p d^{d} k P_{\mu \alpha}^{l a^{\prime}}(p) P_{\nu \beta}^{m b^{\prime}}(p+k) P_{\rho \sigma}^{c c^{\prime}}(k) V^{a^{\prime} b^{\prime} c^{\prime} \alpha \beta \sigma}(-p, p+k,-k) e^{-i k(x-z)} .
$$

A integral (6.45) tem a forma da Eq. (C.8) e podemos concluir que

$$
\int \frac{d^{d} p}{(2 \pi)^{d}} P_{\mu \alpha}^{l a^{\prime}}(p) P_{\nu \beta}^{m b^{\prime}}(p+k) P_{\rho \sigma}^{c c^{\prime}}(k) V^{a^{\prime} b^{\prime} c^{\prime} \alpha \beta \sigma}(-p, p+k,-k)
$$

é igual a contribuição do diagrama da Fig. 6.6(a) no espaço de momento. Note que esse diagrama, no espaço de momento, é equivalente a um diagrama de um loop com um vértice $\left(A_{\mu}^{l}(x) A_{\nu}^{m}(x) A_{\rho}^{b} A_{\sigma}^{c}\right)$ cuja regra é $2 \delta^{l b} \delta^{m c} I_{\mu \nu \rho \sigma}$.

No entanto, estamos interessados em diagramas com o campo composto $h_{\mu \nu}^{a}$, logo o vértice mais adequado seria

$$
\left(h_{\mu \nu}^{a}(x) A_{\rho}^{b} A_{\sigma}^{c}\right)=g f^{a l m}\left(A_{\mu}^{l}(x) A_{\nu}^{m}(x) A_{\rho}^{b} A_{\sigma}^{c}\right)=2 g f^{a b c} I_{\mu \nu \rho \sigma} .
$$

\footnotetext{
${ }^{13}$ Para considerar a conservação de momento, formalmente, consideramos a função delta $(2 \pi)^{d} \delta(p-q-k)$.
} 


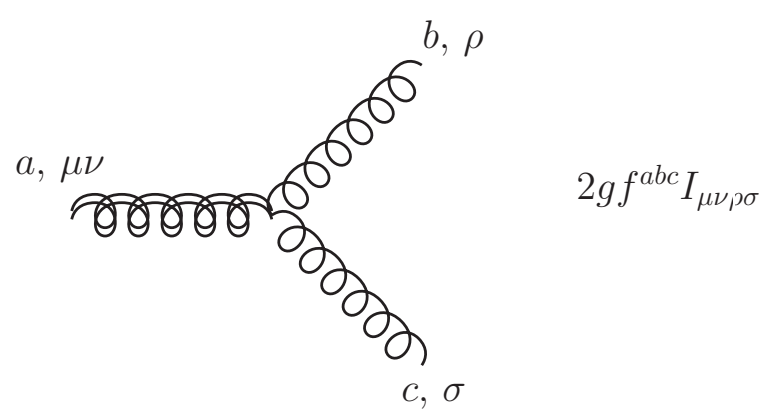

Figura 6.7: Regra de Feynman (estendida) para o vértice $\left(h_{\mu \nu}^{a} A_{\rho}^{b} A_{\sigma}^{c}\right)$. O campo composto $h$ é representado por molas duplas.

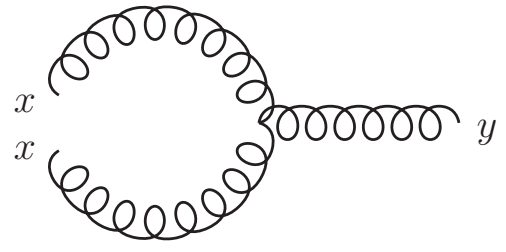

$(a)$

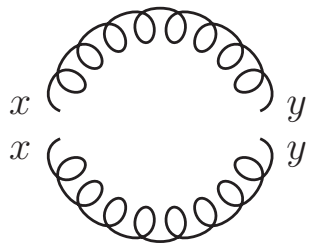

(b)

Figura 6.8: O diagrama (a) é a correção ao nível de um loop para $\left\langle 0\left|T h_{\mu \nu}^{a} A_{\rho}^{b}\right| 0\right\rangle$ e o diagrama (b) para $\left\langle 0\left|T h_{\mu \nu}^{a} h_{\rho \sigma}^{b}\right| 0\right\rangle$.

Com essa regra de Feynman ${ }^{14}$ podemos obter diagramas com o campo composto $h_{\mu \nu}^{a}$ externo que são computados como diagramas usuais do formalismo de segunda ordem; não sendo mais necessário fazer uma análise do comportamento das funções de Green pela contração de pernas externas.

Usando a regra de Feynman estendida na Fig. 6.7 para o vértice auxiliar ( $h A A$ ) podemos obter diagramas (computados como diagramas usuais do formalismo de segunda ordem), como na Fig. 6.8, para correlações com o campo composto $h_{\mu \nu}^{a}$ como as que aparecem no lado direito das Eqs. (3.64b) e (3.64c). Em vez do cálculo de diagramas pinched podemos usar esses diagramas auxiliares que são totalmente equivalentes.

\section{DiagRAMA PINCHED $\left\langle h_{\mu \nu}^{a} A_{\rho}^{b}\right\rangle$}

O lado direito da identidade (3.64b) no nível de um loop usando a regra de Feynman estendida da Fig. 6.7 é dado pelo diagrama 6.8(a). Podemos usar o mesmo procedimento realizado para

\footnotetext{
${ }^{14}$ Note que a regra de Feynman para o vértice $(h A A)$ é igual a regra do vértice $(H A A)$ a menos de um fator de $2 i$. Isso pode entendido através da identidade estrutural $\langle 0|T H A A| 0\rangle=\langle 0|T h A A| 0\rangle$ que no nível árvore é equivalente a $2 i(H A A)=(h A A)$, onde $2 i$ vem do propagador de $H$ que não é amputado.
} 
computar o propagador misto $H A$, no entanto, note que o diagrama da Fig. 6.8(a) é igual a $a^{15}$

$$
\left(\frac{1}{2} \int \frac{d^{d} p}{(2 \pi)^{d}}\left(2 i \mathcal{V}_{\mu \nu \alpha \beta}^{a c d}\right) P_{c c^{\prime}}^{\alpha \pi}(p) P_{d d^{\prime}}^{\beta \tau}(q) V_{\pi \tau \rho^{\prime}}^{c^{\prime} d^{\prime} b^{\prime}}(-p, q,-k)\right) P_{\rho^{\prime} \rho}^{b^{\prime} b}(k),
$$

onde escrevemos a regra para o vértice $(h A A)$ como $2 i \mathcal{V}_{\mu \nu \alpha \beta}^{a c d}$.

Usando a definição do propagador do campo auxiliar $\left\langle H_{\mu \nu}^{a}(-k) H_{\mu^{\prime} \nu^{\prime}}^{a^{\prime}}(k)\right\rangle=2 i I_{\mu \nu \mu^{\prime} \nu^{\prime}} \delta^{a a^{\prime}}$, podemos escrever a integral (6.48) como

$$
\left\langle H_{\mu \nu}^{a}(-k) H^{a^{\prime} \mu^{\prime} \nu^{\prime}}(k)\right\rangle\left(\frac{1}{2} \int \frac{d^{d} p}{(2 \pi)^{d}} \mathcal{V}_{\mu^{\prime} \nu^{\prime} \alpha \beta}^{a^{\prime} c d} P_{c c^{\prime}}^{\alpha \pi}(p) P_{d d^{\prime}}^{\beta \tau}(q) V_{\pi \tau \rho^{\prime}}^{c^{\prime} d^{\prime} b^{\prime}}(-p, q,-k)\right) P_{\rho^{\prime} \rho}^{b^{\prime} b}(k),
$$

onde a integral pode ser identificada como a autoenergia mista $\Pi_{\mu^{\prime} \nu^{\prime} \rho^{\prime}}^{a^{\prime}}(k)$ dada na Eq. (6.21), ou seja, a Eq. (6.49) é igual a Eq. (6.30). Então, o diagrama da Fig. 6.8(a) é igual ao diagrama da Fig. 6.4 e a sua contribuição definida na Eq. (6.48) obviamente leva o mesmo resultado da Eq. (6.31) para o propagador misto $H A$.

\section{DiAGRAMA PINCHED $\left\langle h_{\mu \nu}^{a} h_{\rho \sigma}^{b}\right\rangle$}

A única contribuição para o lado direito da identidade (3.64c) no nível de um loop é o diagrama na Fig. 6.8(b). Usando as regras de Feynman para o formalismo diagonal e a regra estendida na Fig. 6.7, o diagrama da Fig. 6.8(b) é igual a ${ }^{16}$

$$
\frac{1}{2} \int \frac{d^{d} p}{(2 \pi)^{d}}\left(2 i \mathcal{V}_{\mu \nu \alpha \beta}^{a c d}\right) P_{c c^{\prime}}^{\alpha \pi}(p) P_{d d^{\prime}}^{\beta \tau}(q)\left(2 i \mathcal{V}_{\pi \tau \rho \sigma}^{\mathcal{c}^{\prime} d^{\prime} b}\right)
$$

onde voltamos a escrever a regra para o vértice $(h A A)$ como $2 i \mathcal{V}_{\mu \nu \alpha \beta}^{a c d}$.

Usando a definição do propagador do campo auxiliar $H_{\mu \nu}^{a}$ reescrevemos a integral (6.50) como

$$
\left\langle H_{\mu \nu}^{a}(-k) H^{a^{\prime} \mu^{\prime} \nu^{\prime}}(k)\right\rangle\left(\frac{1}{2} \int \frac{d^{d} p}{(2 \pi)^{d}} \mathcal{V}_{\mu^{\prime} \nu^{\prime} \alpha \beta}^{a^{\prime} c d} P_{c c^{\prime}}^{\alpha \pi}(p) P_{d d^{\prime}}^{\beta \tau}(q) \mathcal{V}_{\pi \tau \rho^{\prime} \sigma^{\prime}}^{c^{\prime} d^{\prime} b^{\prime}}\right)\left\langle H^{b^{\prime} \rho^{\prime} \sigma^{\prime}}(-k) H_{\rho \sigma}^{b}(k)\right\rangle
$$

A integral na equação acima é igual a autoenergia do campo auxiliar definida na Eq. (6.33), tal que, a equação acima é igual a Eq. (6.40). Ou seja, o diagrama da Fig. 6.8(b) é igual ao diagrama da Fig. 6.5. Logo, a computação da integral (6.50) obviamente leva o mesmo resultado encontrado para o propagador de $H$ na Eq. (6.38).

\subsubsection{Resultados para o formalismo diagonal}

Pelos resultados obtidos verifica-se que as identidades (3.64b) e (3.64c) são válidas no nível de um loop para qualquer dimensão e em qualquer calibre. Diferentemente do artigo $[15,16]$ que

\footnotetext{
${ }^{15}$ Note que é igual a Eq. (6.46), obtida pela análise mais elementar, com um fator extra de $g f^{a l m}$. Além disso, vale mencionar que o fator de simetria é obtido de acordo com os procedimentos usuais, nesse caso sendo igual a 1/2. O fator de simetria do diagrama original na Fig. 6.6(a) é igual a 1, porém note que no caso do diagrama da Fig. 6.8(a), temos um fator de 2 extra do vértice auxiliar $\left(h_{\mu \nu}^{a} A_{\alpha}^{c} A_{\beta}^{d}\right)$ cancelando o novo fator de simetria de $1 / 2$ e resultando no mesmo fator do diagrama original.

${ }^{16}$ Nessa caso também temos o fator de simetria de $1 / 2$, porém temos dois vértices auxiliares que implica num fator geral de $1 / 2 \times 2 \times 2=2$ que é igual ao fator de simetria do diagrama original da Fig. 6.6(b). Isso mostra que o vértice auxiliar pode ser considerado como uma regra de Feynman usual não sendo meramente um vértice $a d$ hoc.
} 
só mostrou a verificação pela comparação dos resultados explícitos conseguimos mostrar que a equivalência pode ser vista ainda no nível do integrando. Ressaltamos que a verificação explícita como nos artigos $[15,16]$ também foi realizada, porém a igualdade a nível de integrando é bem evidente.

Embora trivial, resta ainda considerar a verificação no nível árvore: para a identidade (3.64b), o lado esquerdo é zero já que não temos propagadores mistos; já o esquerdo só tem contribuições de ordem acima de $g$, portanto, no nível árvore, ambos lados são nulos. Já no lado direito da identidade (3.64c), ao nível de árvore, temos somente o termo de contato $2 i I \delta(x-y)$ que no espaço de momento é igual ao propagador do campo auxiliar $H_{\mu \nu}^{a}$ demostrando explicitamente a identidade (3.64c) no nível árvore.

Na próxima seção mostraremos que a verificação das identidades obtidas para gravitação que são muito mais complicadas. A análise dos diagramas pinched é a mesma, porém os cálculos dos diagramas são mais complexos; a base tensorial usada chega a até 22 tensores tornando os resultados gerais muito menos claros.

\subsection{Gravitação}

Nessa seção vamos verificar as identidades (5.44) e (5.47) até um loop como feito no artigo [16]. Antes reescrevemos as identidades (5.44) e (5.47) de forma explícita como

$$
\begin{gathered}
\left\langle 0\left|T H_{\mu \nu}^{\lambda}(x) \phi^{\pi \tau}(y)\right| 0\right\rangle=\kappa\left\langle 0\left|T\left[M^{-1}[\eta+\kappa \phi(x)]-M^{-1}(\eta)\right]_{\mu \nu \alpha \beta}^{\lambda \rho} \phi_{, \rho}^{\alpha \beta}(x) \phi^{\pi \tau}(y)\right| 0\right\rangle, \\
\left\langle 0\left|T H_{\mu \nu}^{\lambda}(x) H_{\pi \tau}^{\rho}(y)\right| 0\right\rangle=i \kappa^{2}\left\langle 0\left|T\left(M^{-1}\right)_{\mu \nu \pi \tau}^{\lambda \rho}[\eta+\kappa \phi(x)]\right| 0\right\rangle \delta(x-y)+\left\langle 0\left|T \Delta_{\mu \nu}^{\lambda}(x) \Delta_{\pi \tau}^{\rho}(y)\right| 0\right\rangle,
\end{gathered}
$$

lembrando que

$$
\Delta_{\mu \nu}^{\lambda}(x)=\kappa\left[M^{-1}[\eta+\kappa \phi(x)]-M^{-1}(\eta)\right]_{\mu \nu \alpha \beta}^{\lambda} \gamma \phi_{, \gamma}^{\alpha \beta}(x)
$$

\subsubsection{Ordem árvore}

Em menor ordem é fácil ver que essas identidades são válidas. O lado esquerdo da Eq. (6.52) é nulo já que não há presença de propagadores mistos, enquanto o lado direito pode ser obtido perturbativamente usando a expansão (definindo $M(\eta) \equiv m$ )

$$
M^{-1}(\eta+\kappa \phi)-m^{-1}=-\kappa m^{-1} M(\phi) m^{-1}+\kappa^{2} m^{-1} M(\phi) m^{-1} M(\phi) m^{-1}+\cdots,
$$

mostrando que a contribuição de menor ordem é de pelo menos da ordem $\kappa^{2}$. Logo também não contribui na ordem árvore (nesse caso $\kappa$ ) verificando-se a validade da identidade (6.52) nessa ordem.

Para a identidade (6.53), na ordem árvore $\left(\kappa^{2}\right)$, o lado direito só tem uma contribuição do primeiro termo que no espaço de momento é igual a $i \kappa^{2} m^{-1}$, essa contribuição é igual ao propagador do campo auxiliar $H_{\mu \nu}^{\lambda}$ (C.56), ou seja, igual ao lado esquerdo da Eq. (6.53) mostrando que ela é válida em ordem árvore.

$\mathrm{Na}$ próxima seção seguiremos para o nível de um loop, primeiramente mostraremos as correções para os propagadores do formalismo diagonal que são as contribuições para o lado 


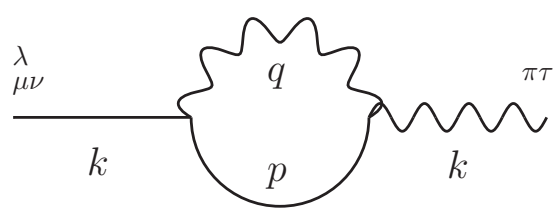

(a)

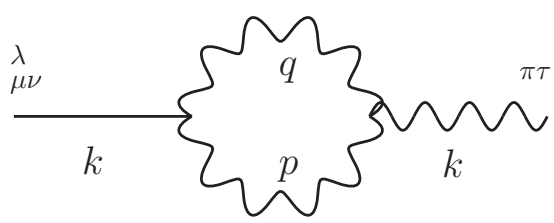

(b)

Figura 6.9: Diagramas de um loop que contribuem para o propagador misto $\left\langle H_{\mu \nu}^{\lambda} \phi^{\pi \tau}\right\rangle$. Usando $p$ como o momento de integração, temos que $q=p+k$.

esquerdo das identidades estruturais. Os diagramas pinched que contribuem para o lado direito também são computados, no entanto, vamos computá-los diretamente no espaço de momento. Uma extensão das regras de Feynman também é possível, mas sai do escopo desse trabalho.

\subsubsection{Correções quânticas de um loop}

\section{Propagador Misto $H \phi$}

Os dois diagramas de um loop para o propagador $\left\langle H_{\mu \nu}^{\lambda} \phi^{\pi \tau}\right\rangle$ estão na Fig. 6.9. Porém, o diagrama na Fig. 6.9(a) não contribui ao usar regularização dimensional já que tem uma estrutura similar a tadpole (já que o propagador interno relativo ao campo auxiliar não depende de momento). Assim a única contribuição não nula é do diagrama da Fig. 6.9(b).

Usando as regras de Feynman definidas no apêndice C.3, o diagrama da Fig. 6.9(b) é dado por

$$
\mathcal{D}_{\mu \nu \mu^{\prime} \nu^{\prime}}^{\lambda \Pi_{\lambda^{\prime}}^{\lambda^{\prime}{ }^{\prime} \tau^{\prime}}} \mu^{\prime} \nu^{\prime}(k) \mathcal{P}^{\pi^{\prime} \tau^{\prime} \pi \tau}(k),
$$

onde $\mathcal{D}_{\mu \nu}^{\lambda \mu^{\prime} \nu^{\prime}} \lambda^{\prime}$ é igual ao propagador do campo auxiliar $H$ definido na Eq. (C.56) e $\mathcal{P}^{\pi^{\prime} \tau^{\prime} \pi \tau}(k)$ é o propagador do gráviton definido na Eq. (C.55).

A autoenergia mista $\Pi_{\lambda^{\prime}}^{\mu^{\prime} \nu^{\prime} \tau^{\prime}}(k)$ é igual a

$$
\frac{1}{2} \int \frac{d^{d} p}{(2 \pi)^{d}} V_{\lambda^{\prime}}^{\mu^{\prime} \nu^{\prime}}{ }_{\alpha_{1} \beta_{1} \alpha_{2} \beta_{2}}(k, p,-q) \mathcal{P}^{\alpha_{1} \beta_{1} \gamma_{1} \theta_{1}}(p) \mathcal{P}^{\alpha_{2} \beta_{2} \gamma_{2} \theta_{2}}(q) V_{\gamma_{1} \theta_{1} \gamma_{2} \theta_{2} \pi^{\prime} \tau^{\prime}}(-p, q,-k),
$$

onde $V_{\lambda}^{\mu \nu}{ }_{\pi \tau \alpha \beta}(p, q, k)$ e $V_{\mu \nu \pi \tau \alpha \beta}(p, q, k)$ são, respectivamente, as regras de Feynman para os vértices $(H \phi \phi)$ e $(\phi \phi \phi)$ definidas nas Eqs. (C.58) e (C.59).

Podemos decompor a integral (6.57) numa base tensorial geral (considerando as simetrias da autoenergia) $\left\{T_{i}^{(H \phi)}\right\}$ cujo tensores são definidos nas Eqs. (D.28), tal que

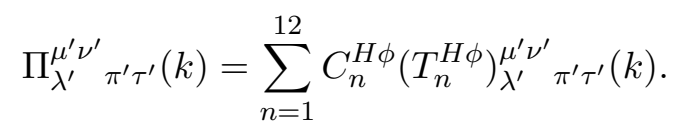

Contraindo a equação acima com os mesmos 12 tensores da base obtemos um sistema de 12 equações lineares que pode ser resolvido usando computação simbólica. O resultado geral (os 12 coeficientes $C_{n}^{H \phi}$ para qualquer $d>2$ e qualquer calibre $\alpha$ ) computado está listado no apêndice E. Para ilustrar o resultado, os coeficientes $C_{n}^{H \phi}$ da Eq. (6.58) para o polo UV ( $d=4-2 \epsilon$ ), no calibre de DeDonder $(\alpha=1)$, é listado na Tabela 6.1 . 


\begin{tabular}{|c|c|c|c|c|c|c|c|c|c|c|c|}
\hline$C_{1}^{H \phi}$ & $C_{2}^{H \phi}$ & $C_{3}^{H \phi}$ & $C_{4}^{H \phi}$ & $C_{5}^{H \phi}$ & $C_{6}^{H \phi}$ & $C_{7}^{H \phi}$ & $C_{8}^{H \phi}$ & $C_{9}^{H \phi}$ & $C_{10}^{H \phi}$ & $C_{11}^{H \phi}$ & $C_{12}^{H \phi}$ \\
\hline$\frac{1}{48}$ & $\frac{1}{4}$ & $-\frac{13}{96}$ & 0 & $-\frac{19}{48}$ & $\frac{3}{16}$ & $-\frac{1}{24}$ & $-\frac{1}{4}$ & $\frac{3}{8}$ & 0 & $-\frac{5}{24}$ & $-\frac{1}{12}$ \\
\hline
\end{tabular}

Tabela 6.1: Polo UV dos coeficientes da autoenergia mista $H \phi$ no calibre de DeDonder, definida na Eq. (6.58), em unidades de $i \kappa k^{2} I_{U V}$.

\begin{tabular}{cccccccccccc}
\hline$P_{1}^{H \phi}$ & $P_{2}^{H \phi}$ & $P_{3}^{H \phi}$ & $P_{4}^{H \phi}$ & $P_{5}^{H \phi}$ & $P_{6}^{H \phi}$ & $P_{7}^{H \phi}$ & $P_{8}^{H \phi}$ & $P_{9}^{H \phi}$ & $P_{10}^{H \phi}$ & $P_{11}^{H \phi}$ & $P_{12}^{H \phi}$ \\
\hline 0 & $\frac{31}{48}$ & $-\frac{9}{32}$ & $-\frac{1}{48}$ & $-\frac{1}{2}$ & $-\frac{11}{48}$ & $\frac{5}{12}$ & $-\frac{3}{4}$ & $\frac{5}{8}$ & 0 & $\frac{19}{48}$ & $-\frac{1}{48}$ \\
\hline
\end{tabular}

Tabela 6.2: Polo UV dos coeficientes do propagador misto $H \phi$ no calibre de DeDonder, definido na Eq. (6.59), em unidades de $i \kappa^{3} I_{U V}$.

A correção de um loop para o propagador é dada pela Eq. (6.56); escrevendo o resultado na mesma base, isso é, decompondo a Eq. (6.56) como

$$
\sum_{n=1}^{12} P_{n}^{H \phi}\left(T_{n}^{H \phi}\right)_{\mu \nu}^{\lambda} \pi \tau
$$

podemos obter os coeficientes pela solução do sistema de 12 equações lineares obtido através da projeção dos tensores da base na Eq. (6.56). Os coeficientes $P_{n}^{H \phi}$ obtidos para o polo UV, no calibre de DeDonder, são listados na Tabela 6.2. O resultado geral para esses coeficientes também estão listados no apêndice E.

Comparando com o resultado do artigo [16, Tabela II e V] temos diferenças ${ }^{17}$ devido a definição das regras de Feynman. O vértice $(H \phi \phi)$ é definido aqui de forma oposta ao usado [16] por isso a Tabela 6.1 e a tabela II tem sinais opostos. Já as tabelas 6.2 e V coincidem (a menos de um fator de $\kappa$ ) pois nossas regras de Feynman são obtidas de $i S$, enquanto as regras do artigo [16] são obtidas de $S$, isso leva a um fator de $i^{2}$ na Eq. (6.56) que junto com o fator de -1 para autoenergia se cancelam.

\section{PROPAGADOR DO CAMPO AUXILIAR $H$}

Para a correção de um loop do propagador do campo auxiliar $H$, no nível de um loop, temos os diagramas da Fig. 6.10. Assim como o diagrama da Fig. 6.9(a), o diagrama na Fig. 6.10(a) não contribui usando regularização dimensional.

O único diagrama não nulo, na Fig. 6.9(b), é igual a

$$
\mathcal{D}_{\mu \nu \mu^{\prime} \nu^{\prime}}^{\lambda \Pi_{\lambda^{\prime}}^{\lambda^{\prime}} \Pi^{\prime}} \underset{\mu^{\prime} \nu^{\prime} \pi^{\prime} \tau^{\prime}}{\lambda}(k) \mathcal{D}_{\pi^{\prime} \tau^{\prime} \pi \tau}^{\rho^{\prime}}, \rho
$$

\footnotetext{
${ }^{17}$ Essas diferenças (exceto os fatores de $\kappa$ ) são devidos a convenções e não alteram a validade da verificação; escolhemos, por consistência, usar as mesmas convenções usadas no caso da teoria de YM. Embora a convenção usada no artigo [16] seja comum para gravitação [6, 32].
} 


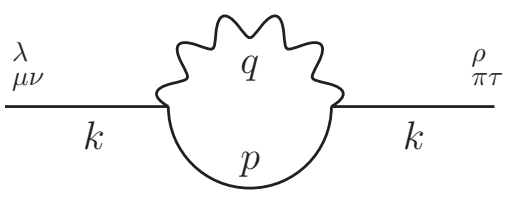

(a)

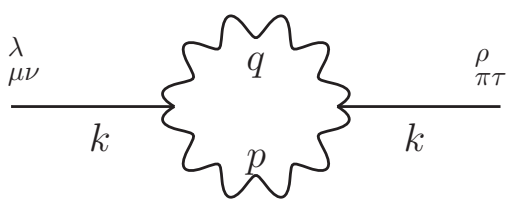

(b)

Figura 6.10: Diagramas de um loop que contribuem para o propagador $\left\langle H_{\mu \nu}^{\lambda} H_{\pi \tau}^{\rho}\right\rangle$.

\begin{tabular}{ccccccccccc}
\hline$C_{1}^{H H}$ & $C_{2}^{H H}$ & $C_{3}^{H H}$ & $C_{4}^{H H}$ & $C_{5}^{H H}$ & $C_{6}^{H H}$ & $C_{7}^{H H}$ & $C_{8}^{H H}$ & $C_{9}^{H H}$ & $C_{10}^{H H}$ & $C_{11}^{H H}$ \\
\hline$-\frac{1}{4}$ & $-\frac{1}{4}$ & $\frac{1}{6}$ & $\frac{7}{12}$ & $-\frac{1}{6}$ & 0 & 0 & 0 & 0 & $\frac{1}{6}$ & $\frac{4}{3}$ \\
\hline$C_{12}^{H H}$ & $C_{13}^{H H}$ & $C_{14}^{H H}$ & $C_{15}^{H H}$ & $C_{16}^{H H}$ & $C_{17}^{H H}$ & $C_{18}^{H H}$ & $C_{19}^{H H}$ & $C_{20}^{H H}$ & $C_{21}^{H H}$ & $C_{22}^{H H}$ \\
\hline$\frac{5}{6}$ & 0 & $-\frac{2}{3}$ & $\frac{4}{3}$ & $\frac{13}{6}$ & $\frac{2}{3}$ & $-\frac{17}{3}$ & -1 & 0 & 0 & 1 \\
\hline
\end{tabular}

Tabela 6.3: Polo UV dos coeficientes da autoenergia $H H$ no calibre de DeDonder, definida na Eq. (6.62), em unidades de $k^{2} I_{U V}$.

onde a autoenergia do campo $H, \Pi_{\lambda^{\prime}}^{\mu^{\prime} \nu^{\prime} \pi^{\prime} \tau^{\prime}}(k)$, é dada por

$$
\frac{1}{2} \int \frac{d^{d} p}{(2 \pi)^{d}} V_{\lambda^{\prime}}^{\mu^{\prime} \nu^{\prime}}{ }_{\alpha_{1} \beta_{1} \alpha_{2} \beta_{2}}(k, p,-q) \mathcal{P}^{\alpha_{1} \beta_{1} \gamma_{1} \theta_{1}}(p) \mathcal{P}^{\alpha_{2} \beta_{2} \gamma_{2} \theta_{2}}(q) V_{\gamma_{1} \theta_{1} \gamma_{2} \theta_{2} \rho^{\prime}}{ }^{\pi^{\prime} \tau^{\prime}}(-p, q,-k) .
$$

Decompondo a integral (6.57) numa base tensorial geral (considerando as simetrias da autoenergia) $\left\{T_{i}^{(H H)}\right\}$ cujo tensores são definidos nas Eqs. (D.29):

$$
\Pi_{\lambda^{\prime}}^{\mu^{\prime} \nu^{\prime} \frac{\pi}{\prime}^{\prime} \tau^{\prime}}(k)=\sum_{n=1}^{22} C_{n}^{H H}\left(T_{n}^{H H}\right)_{\lambda^{\prime}}^{\mu^{\prime} \nu^{\prime} \pi^{\prime} \tau^{\prime}}(k)
$$

e contraindo com os 22 tensores da base obtemos um sistema de 22 equações lineares que pode ser resolvido usando computação simbólica. O resultado geral (os 22 coeficientes $C_{n}^{H H}$ em qualquer dimensão $d>2$ e calibre $\alpha$ ) obtido está listado no apêndice E. O resultado para os coeficientes $C_{n}^{H H}$ da Eq. (6.62) do polo UV $(d=4-2 \epsilon)$ no calibre de DeDonder $(\alpha=1)$ é dado na Tabela 6.3 .

A correção de um loop para o propagador, dada pela Eq. (6.56), pode ser escrita na base tensorial da autoenergia como

$$
\sum_{n=1}^{2} P_{n}^{H H}\left(T_{n}^{H H}\right)_{\mu \nu}^{\lambda \pi \tau},
$$

onde os coeficientes podem ser obtidos pela solução do sistema de 22 equações lineares obtido pela projeção dos tensores da base na Eq. (6.60). Os coeficientes $P_{n}^{H H}$ do polo UV, no calibre de DeDonder, são listados na Tabela 6.4 e os coeficientes no caso geral podem ser encontrado no apêndice E. 


\begin{tabular}{ccccccccccc}
\hline$P_{1}^{H H}$ & $P_{2}^{H H}$ & $P_{3}^{H H}$ & $P_{4}^{H H}$ & $P_{5}^{H H}$ & $P_{6}^{H H}$ & $P_{7}^{H H}$ & $P_{8}^{H H}$ & $P_{9}^{H H}$ & $P_{10}^{H H}$ & $P_{11}^{H H}$ \\
\hline$-\frac{1}{6}$ & $\frac{1}{3}$ & $-\frac{1}{48}$ & $-\frac{5}{12}$ & $\frac{1}{4}$ & 0 & 0 & 0 & 0 & -1 & $-\frac{4}{3}$ \\
\hline$P_{12}^{H H}$ & $P_{13}^{H H}$ & $P_{14}^{H H}$ & $P_{15}^{H H}$ & $P_{16}^{H H}$ & $P_{17}^{H H}$ & $P_{18}^{H H}$ & $P_{19}^{H H}$ & $P_{20}^{H H}$ & $P_{21}^{H H}$ & $P_{22}^{H H}$ \\
\hline$-\frac{5}{6}$ & $-\frac{2}{3}$ & 0 & $\frac{2}{3}$ & $-\frac{5}{2}$ & $-\frac{1}{3}$ & 5 & $-\frac{3}{2}$ & 0 & 0 & $\frac{17}{24}$ \\
\hline
\end{tabular}

Tabela 6.4: Polo UV dos coeficientes do propagador $H H$ no calibre de DeDonder, definido na Eq. (6.63), em unidades de $\kappa^{4} k^{2} I_{U V}$.

Comparando com o resultado do artigo [16, Tabela IV e VII] obtemos sinais opostos para o propagador, porém agora a autoenergia é igual. Isso é consistente com a divergência encontrada para o caso do propagador misto.

\subsubsection{Correlações do campo composto $\Delta_{\mu \nu}^{\lambda}(x)$}

Diferentemente da teoria de YM, onde as correlações com o campo composto $h_{\mu \nu}^{a}(x)$ de um loop eram iguais a diagramas pinched, na gravitação o lado direito das identidades estruturais é igual a uma combinação linear de diagramas pinched.

A princípio deveríamos considerar todas as combinações individualmente complicando imensamente os cálculos, porém no artigo [16] usamos uma abordagem ${ }^{18}$ mais eficiente em que todas essas combinações são obtidas diretamente dos diagramas pinched usando um tensor independente dos campos (e derivadas) $\mathcal{M}$.

IDENTIDADE EQ. (5.44)

O lado direito da Eq. (5.44), na ordem de um loop $\left(\kappa^{2}\right)$, é igual a

$$
-\kappa^{2}\left\langle 0\left|T\left[m^{-1} M(\phi) m^{-1}\right]_{\mu \nu \alpha \beta}^{\lambda \gamma}(x) \phi_{, \gamma}^{\alpha \beta}(x) \phi^{\pi \tau}(y)\right| 0\right\rangle,
$$

onde usamos a expansão (6.55). Conforme [16], podemos fatorar o campo $\phi$ do tensor $M(\phi)$, definindo o tensor $\mathcal{M}$ como ${ }^{19}$

$$
\kappa \mathcal{M}_{\mu \nu \alpha \beta \rho \sigma}^{\lambda \gamma} \phi^{\rho \sigma} \equiv-\kappa\left[m^{-1} M(\phi) m^{-1}\right]_{\mu \nu \alpha \beta}^{\lambda \gamma} .
$$

Assim, podemos reescrever a Eq. (6.64) como

$$
\kappa^{2}\left\langle 0\left|T \mathcal{M}_{\mu \nu \alpha \beta \rho \sigma}^{\lambda \gamma} \phi^{\rho \sigma}(x) \phi_{, \gamma}^{\alpha \beta}(x) \phi^{\pi \tau}(y)\right| 0\right\rangle=\kappa^{2} \mathcal{M}_{\mu \nu \alpha \beta \rho \sigma}^{\lambda \gamma}\left\langle 0\left|T \phi^{\rho \sigma}(x) \phi_{, \gamma}^{\alpha \beta}(x) \phi^{\pi \tau}(y)\right| 0\right\rangle .
$$

Pela fatoração, como vemos na Eq. (6.66), podemos agora computar todas contribuições em termo da correlação $\left\langle 0\left|T \phi^{\rho \sigma}(x) \phi_{, \gamma}^{\alpha \beta}(x) \phi^{\pi \tau}(y)\right| 0\right\rangle$, sendo análoga a $\left\langle 0\left|T A_{\mu}^{l}(x) A_{\nu}^{m}(x) A_{\rho}^{b}(y)\right| 0\right\rangle$

\footnotetext{
${ }^{18}$ Essa abordagem valeria a princípio somente para um loop, porém pela a expansão Eq. (6.55) fica claro que podemos estendê-la para ordem superiores sem dificuldades.

${ }^{19} \mathrm{~A}$ forma explícita do tensor $\mathcal{M}$ não é dada aqui, porém a sua obtenção é simples já que $M(\phi)$ é linear, ou seja, $M(\phi)=\frac{\partial M(\phi)}{\partial \phi} \cdot \phi$, tal que, $\mathcal{M}=-m^{-1} \cdot \frac{\partial M(\phi)}{\partial \phi} \cdot m^{-1}$.
} 


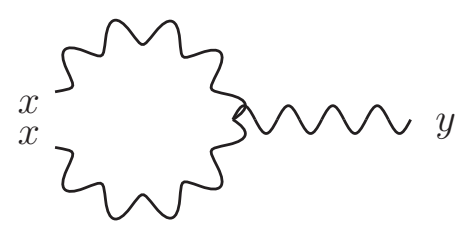

Figura 6.11: Diagrama pinched de um loop que contribui para o lado direito da Eq. (6.52).

da teoria de YM. Na Fig. 6.11, temos um exemplo de diagrama pinched que contribui para $\left\langle 0\left|T \phi^{\rho \sigma}(x) \phi^{\alpha \beta}(x) \phi^{\pi \tau}(y)\right| 0\right\rangle$ que é análogo ao diagrama da Fig. 6.6(a), isso significa que a análise feita na seção 6.1 .4 vale para gravitação fazendo as substituições necessárias.

Por exemplo, o diagrama pinched da Fig. 6.11 no espaço de momento pode ser obtido a partir do resultado encontrado para seu análogo da Fig. 6.6(a), na Eq. (6.46) como

$$
\int \frac{d^{d} p}{(2 \pi)^{d}} \mathcal{P}_{\alpha \beta \alpha^{\prime} \beta^{\prime}}(p) \mathcal{P}_{\rho \sigma \rho^{\prime} \sigma^{\prime}}(q) V^{\rho^{\prime} \sigma^{\prime} \alpha^{\prime} \beta^{\prime} \pi^{\prime} \tau^{\prime}}(q,-p,-k) \mathcal{P}_{\pi^{\prime} \tau^{\prime} \pi \tau}(k)
$$

onde $q=p+k ; \mathcal{P}_{\mu \nu \alpha \beta}(p)$ é o propagador do campo $\phi^{\mu \nu}$ e $V^{\alpha \beta \mu \nu \pi \tau}(p, q, k)$ é igual ao vértice cúbico $(\phi \phi \phi)$ ambos dados explicitamente no apêndice C.3.

A correlação (6.64) tem uma forma similar a Eq. (6.67) sendo dada por ${ }^{20}$

$$
-i \kappa^{2} \mathcal{M}_{\mu \nu \alpha \beta \rho \sigma}^{\lambda \gamma} \int \frac{d^{d} p}{(2 \pi)^{d}} p_{\gamma} \mathcal{P}^{\alpha \beta \alpha^{\prime} \beta^{\prime}}(p) \mathcal{P}^{\rho \sigma \rho^{\prime} \sigma^{\prime}}(q) V_{\rho^{\prime} \sigma^{\prime} \alpha^{\prime} \beta^{\prime} \pi^{\prime} \tau^{\prime}}(q,-p,-k) \mathcal{P}^{\pi^{\prime} \tau^{\prime} \pi \tau}(k) .
$$

Computando essa integral obtemos o mesmo resultado encontrado para a correção do propagador misto $\left\langle H_{\mu \nu}^{\lambda} \phi^{\pi \tau}\right\rangle$ obtido a partir da Eq. (6.56), tal que, a identidade (5.44) é verificada até um loop num calibre geral $\alpha$ e numa dimensão qualquer $d>2$.

\section{IDENTIDADE EQ. (5.47)}

O lado direito da identidade (5.47), na ordem de um loop $\left(\kappa^{4}\right)$, é igual a

$$
\kappa^{4} \mathcal{M}_{\mu \nu \pi_{1} \tau_{1} \rho_{1} \sigma_{1}}^{\lambda \gamma_{1}} \mathcal{M}_{\pi \tau}^{\rho} \gamma_{2} \tau_{2} \tau_{2} \rho_{2} \sigma_{2}\left\langle 0\left|T \phi^{\rho_{1} \sigma_{1}}(x) \phi_{, \gamma_{1}}^{\pi_{1} \tau_{1}}(x) \phi^{\rho_{2} \sigma_{2}}(y) \phi_{, \gamma_{2}}^{\pi_{2} \tau_{2}}(y)\right| 0\right\rangle
$$

onde já fatoramos o campo $\phi$ usando a Eq. (6.65).

O diagrama pinched que contribui, no nível de um loop, para a correlação da Eq. (6.69) mostrado na Fig. 6.12 é análogo ao diagrama na Fig. 6.6(b) visto na teoria de YM, enquanto o diagrama da Fig. 6.12(b) não contribui. Na teoria de YM temos um diagrama análogo [15, Fig. 5(d)] que foi omitido anteriormente já que (como o diagrama da Fig. 6.12(b)) não contribui usando regularização dimensional.

A Eq. (6.69), no espaço de momento, é dada por [16]

$$
-\kappa^{4} \mathcal{M}_{\mu \nu \pi_{1} \tau_{1} \rho_{1} \sigma_{1}}^{\lambda} \Sigma_{\gamma_{1}}^{\pi_{1} \tau_{1} \rho_{1} \sigma_{1} \pi_{2} \tau_{2} \rho_{2} \sigma_{2}} \mathcal{M}_{\pi_{2} \tau_{2} \pi \tau \rho_{2} \sigma_{2}}^{\gamma_{2}} \rho
$$

onde definimos

$$
\Sigma_{\gamma_{1}}^{\pi_{1} \tau_{1} \rho_{1} \sigma_{1} \pi_{2} \tau_{2} \rho_{2} \sigma_{2}} \equiv \int \frac{d^{d} p}{(2 \pi)^{d}}\left[\mathcal{P}^{\pi_{1} \tau_{1} \rho_{2} \sigma_{2}}(p) \mathcal{P}^{\rho_{1} \sigma_{1} \pi_{2} \tau_{2}}(q) p_{\gamma_{1}}-q_{\gamma_{1}} \mathcal{P}^{\rho_{1} \sigma_{1} \rho_{2} \sigma_{2}}(p) \mathcal{P}^{\pi_{1} \tau_{1} \pi_{2} \tau_{2}}(q)\right] q_{\gamma_{2}} .
$$

\footnotetext{
${ }^{20} \mathrm{O}$ resultado obtido é igual ao obtido no artigo [16, Eq. A.15] a menos de um fator de $\kappa$. Essa divergência já era esperada, pois como já mencionamos antes no artigo há uma pequena inconsistência no uso de $h^{\mu \nu}$ e $\phi^{\mu \nu}$.
} 


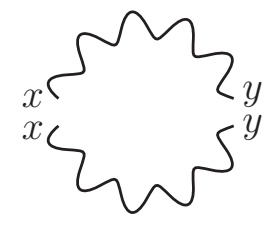

(a)
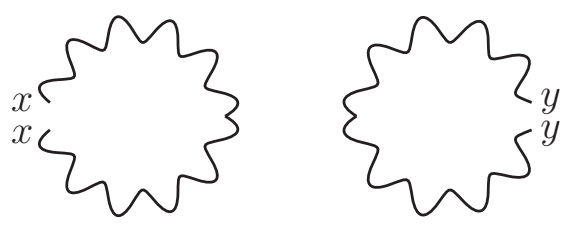

(b)

Figura 6.12: Diagramas pinched que contribuem, no nível de um loop, para o lado direito da Eq. (6.53).

O primeiro termo do integrando acima é a contribuição do diagrama pinched da Fig. 6.16(a), enquanto o segundo é uma permutação dele. Nesse caso, a permutação não é igual devido a presença das derivadas na correlação; então, o fator de simetria do diagrama da Fig. 6.16(a) é igual a um (diferente do análogo da teoria de YM que era dois).

Computando a Eq. (6.70) obtemos o mesmo resultado encontrado para a correção do propagador $H$ obtido a partir da Eq. (6.60). Confirmando a identidade (5.47) até um loop para qualquer calibre $\alpha$ e dimensão $d>2$. 



\section{CAPÍTULO 7}

\section{CONSIDERAÇÕes Finais}

Mostramos a quantização da teoria de YM e da gravitação em diferentes formulações: o formalismo de segunda e primeira ordem e o formalismo diagonal. $\mathrm{O}$ formalismo de segunda ordem sendo igual a formulação usual, enquanto o formalismo de primeira ordem é uma formulação alternativa em que há uma simplificação dos vértices da teoria pela presença de um campo auxiliar. Contundo, temos também a presença de propagadores mistos entre o campo auxiliar e o campo de calibre. No formalismo diagonal, derivado do formalismo de primeira ordem, tais propagadores são eliminados, no entanto o caráter de primeira ordem também é perdido.

Para gravitação, onde há infinitos vértices na formulação usual, o formalismo diagonal ainda continua relevante já que a simplificação é mais significativa. Invés de infinitos vértices, temos apenas três vértices cúbicos. Sendo essa uma das razões que o estudo perturbativo nesse formalismo foi priorizado aqui.

O estudo da equivalência desses diferentes formalismos já foi amplamente considerado na literatura. Como vimos é fácil ver que esses formalismos são equivalentes no nível clássico, tanto para teoria de YM $[4,5]$, quanto para gravitação $[5,6]$. O mesmo vale quando consideramos a equivalência do funcional gerador $Z$ sem fontes nos diferentes formalismos que denominamos de equivalência quântica parcial, pois não se considera as fontes.

Como as fontes têm um papel relevante na computação das funções de Green a partir do funcional gerador $Z$ elas precisam ser consideradas para garantir a equivalência quântica. Esse foi nosso principal objetivo nesse trabalho, demostramos de acordo com $[15,16]$ que a equivalência quântica com fontes é válida para todos formalismos, sem considerar os campos de matéria, usando shifts no campo auxiliar.

Esses shifts foram obtidos usando uma condição induzida pelos shifts usados na demonstração da equivalência parcial dos formalismos, onde os shifts considerados eram em relação a configuração clássica do campo auxiliar. Essa condição foi estendida para o caso com fontes bastando notar que as fontes quânticas adicionadas na quantização poderiam ser consideradas como fontes clássicas. Vale ressaltar que em [16] essa condição já tinha sido levantada na comparação entre os shifts usados para teoria de YM e para gravitação notando que o termo que aparece com a fonte é 
igual ao termo obtido com a equação de Euler-Lagrange.

A configuração clássica obtida pelas equações de Euler-Lagrange então considerava a lagrangiana original junto com fontes clássicas iguais as fontes introduzidas na quantização. Como esperado os shifts obtidos usando esse ansatz concordavam com os shift usados nos artigos $[15,16]$ e assim demostramos a equivalência quântica dos três formalismos para teoria de YM e para gravitação.

A seguir vimos que a equivalência quântica implica num conjunto de identidades estruturais que relacionam funções de Green no formalismo de primeira ordem (ou diagonal) com funções de Green computados no formalismo de segunda ordem. Em especial, vimos que as funções de Green com campos de calibre (o campo físico) externos são iguais em todos formalismos, sendo essa uma das identidades estruturais mais relevantes já que mostra explicitamente a equivalência quântica dos formalismo considerados.

As demais identidades relacionavam o campo auxiliar no formalismo de primeira ordem (diagonal) com sua configuração clássica no formalismo de segunda ordem, dando uma interpretação física do campo auxiliar [15]. As configurações clássicas desses campos auxiliares no nível quântico são iguais a campos compostos já que possuem produtos de campos de calibre no mesmo ponto do espaço-tempo. As correlações com campos compostos levam a divergências de origem diferente das usuais divergências UV encontradas nos diagramas com loops [15]. Essas divergências são essenciais para a verificação das identidades estruturais já que são estas mesmas que cancelam as divergências usuais computadas no formalismo de primeira ordem.

Finalmente, verificamos explicitamente algumas das identidades estruturais derivadas até no nível de um loop usando computação simbólica. Antes foi necessário fazer uma análise das divergências que surgem com os campos compostos que diagramaticamente são dadas por diagramas pinched. Esses diagramas, em um loop, foram computados considerando diagramas de ordem árvore cujas pernas externas convergiam para o mesmo ponto do espaço-tempo levando a diagramas semelhantes a loops.

Para teoria de YM, mostramos que esses diagramas podiam ser obtidos usando o vértice auxiliar $\left(A_{\mu}^{l}(x) A_{\nu}^{m}(x) A_{\alpha}^{b} A_{\beta}^{c}\right)$ e assim estendendo as regras de Feynman, tal que, conseguimos comparar os resultados no nível de integrando. Com essa extensão do artigo original [15] fica claro a razão pela qual os resultados explícitos valem para qualquer dimensão e qualquer calibre.

Além desse procedimento ser mais elementar, a extensão das regras de Feynman para computação de diagramas com campos compostos externos facilita os cálculos pois tais diagramas podem ser computados diretamente, incluindo o fator de simetria. Isso revela que tal procedimento não é meramente $a d$ hoc, embora a origem formal através do funcional gerador $Z$ ainda não é claro.

Para a gravitação seguimos a Ref. [16] fazendo uma verificação puramente explícita até o nível de um loop chegando a resultados semelhantes, embora com algumas diferenças devido a convenções diferentes. Mesmo assim conseguimos mostrar, como no artigo, que as identidades são válidas até o nível de um loop em qualquer calibre e qualquer dimensão $d>2$. A verificação no nível de integrando das identidades no contexto da gravitação também pode ser considerada usando a abordagem mencionada anteriormente. 
Embora a questão da equivalência quântica pareça estar bem resolvida nos casos considerados aqui, ainda podemos levantar algumas questões em relação a adição de campos de matéria. No caso da teoria de YM parece que a adição de tais campos não altera os argumentos usados nesse trabalho para a equivalência quântica nos diferentes formalismos estudados, porém na gravitação isso é mais delicado. É bem conhecido que não há equivalência, até no nível clássico, entre o formalismo de primeira ordem e o formalismo usual da gravitação com a adição de campos fermiônicos $[4,13]$. Portanto, o estudo da equivalência com a presença de campos de matéria na gravitação ainda é um tópico interessante de estudo.

Diante diversas analogias entre a gravitação e a teoria de YM, incluindo seus formalismos alternativos, a razão da quebra de equivalência na presença de campos de matéria somente no caso da gravitação é de certa forma misteriosa. Ainda que tecnicamente seja fácil de entender tal diferença, uma vez que no caso da gravitação com campos fermiônicos temos uma divergência óbvia pelo surgimento de torção na conexão afim (no formalismo de Palatini), isso pode ser um indicativo de uma diferença intrínseca entre o acoplamento do campo de calibre e de um campo de matéria numa teoria de calibre usual, como é o caso da teoria de YM, e na gravitação. Ou seja, o gráviton não se acopla da mesma forma que um fóton (ou glúon da cromodinâmica quântica) a um campo de matéria.

Contudo, na Ref. [35] mostra-se que para o acoplamento de um campo de matéria que não depende da conexão a equivalência ainda é válida. Sendo assim, o estudo do acoplamento de campos de matéria com essa propriedade no formalismo de primeira ordem da gravitação pode ser considerado futuramente sem qualquer necessidade de alterações na teoria, diferentemente do que é feito em Refs. como [36, 37] que sugerem alterações à gravitação.

Além disso, existem algumas dificuldades na definição da ação efetiva $\Gamma$ nos formalismos de primeira ordem da teoria de YM e da gravitação. Um entendimento melhor da ação efetiva em formalismos com a presença de propagadores mistos nos permitiria ver a equivalência quântica de uma perspectiva diferente. No artigo [8] encontra-se a renormalização da teoria de YM no formalismo de primeira usando o formalismo de Becchi, Rouet, Stora, and Tyutin (BRST) que é baseado na invariância da ação efetiva $\Gamma$, mas uma análise ainda mais elementar (e explícita) sobre a ação efetiva $\Gamma$ nos formalismos de primeira ordem pode ser considerada. Em especial, o estudo das ações efetiva generalizadas (tais como os funcionais generalizados $Z$ obtidos) talvez possam esclarecer a origem da regra de Feynman auxiliar usada nesse trabalho.

Outro desdobramento interessante do nosso trabalho seria considerar a formulação de primeira ordem (e diagonal) da gravitação no formalismo de teoria de campos à temperatura finita. Ainda não é claro se há uma equivalência quando consideramos $T>0$. Considerando que as contribuições do determinante $\operatorname{det}^{-1 / 2} M(h)$ podem não ser nulas — o argumento que usa a regularização dimensional não é mais válido à temperatura finita, embora seja possível a existência de uma razão mais elementar para tal anulação - podemos ter que considerar contribuições extras ao usar o formalismo de primeira ordem para ter concordância com o formalismo usual. 



\section{APÊNDICE A}

\section{TeOrias DE CALiBre}

Neste apêndice é revisado o conceito de transformações de calibre e as teorias que são invariantes sobre tais transformações, as teorias de calibre, em especial a teoria de YM. Iremos derivar a lagrangiana para a teoria de YM na sua forma tradicional que equivale a formulação de segunda ordem dada na Eq. (2.1).

O estudo da teoria de YM é interessante já que podemos fazer diversos paralelos com a gravitação que também pode ser tratada como uma teoria de calibre ${ }^{1}$. Além disso, a gravitação é análoga a teoria de YM em alguns aspectos como é o caso das formulações de primeira ordem (diagonal) dessas teorias.

Abaixo temos uma tabela comparando os elementos que compõem as teorias de calibre com os da gravitação:

\begin{tabular}{cc}
\hline Teorias de Calibre & Gravitação \\
\hline Transformação de calibre & Transformação de coordenadas \\
$\Phi^{\prime}=g \Phi$ & $x^{\mu} \rightarrow x^{\prime \mu}(x)$ \\
\hline Campo de calibre & Conexão afim \\
$A_{\mu}$ & $\Gamma_{\mu \nu}^{\lambda}$ \\
\hline Tensor campo de intensidade & Tensor de Curvatura de Riemann \\
$F_{\mu \nu}$ & $R_{\mu \nu \lambda}^{\kappa}$ \\
\hline
\end{tabular}

Tabela A.1: Paralelos entre teorias de calibre e a gravitação

\footnotetext{
${ }^{1}$ Isso aparece na literatura antes mesmo da própria ideia de teorias de calibre surgir, e. g. [41]. Uma abordagem mais atual (e adequada) é encontrada em [42]. Esse ainda é um campo muito produtivo e diversas outras abordagem podem ser consideradas. Em [43], temos uma formulação de primeira ordem que leva a equações com uma forma semelhante as das teoria de YM.
} 


\section{A.1 Transformação de calibre}

Uma transformação de calibre é definida como uma transformação local que pertence a certos grupos de $\mathrm{Lie}^{2}$. No caso de teorias de YM usualmente são considerados somente os grupos de Lie compactos que garantem que a energia seja limitada por baixo [45]. Ou seja, teorias de YM com grupo de calibre compactos possuem uma hamiltoniana positiva definida.

Considere um campo $\Phi$ que se transforma pela ação do grupo de Lie $G$, tal que,

$$
\Phi^{\prime}=g\left(x^{\mu}\right) \Phi,
$$

onde $g$ depende do ponto do espaço tempo $x^{\mu}$. A transformação da Eq. (A.1) é uma transformação de calibre, ou seja, uma transformação local sob um grupo de lie $G$, denominado de grupo de calibre.

Os grupos de calibres podem ser abelianos, caso seus elementos comutem. É o caso do grupo $U(1)$. Ou podem ser não abelianos, ou seja, seus elementos não comutam, esse é o caso da maior parte dos grupos de Lie. Um exemplo notável de grupo não abeliano é o $S U(N)$, especialmente na física, pois é compacto, simplesmente conexo e sua álgebra de Lie é simples. Essas são apenas alguns exemplos de suas propriedades relevantes para física, principalmente no estudo dentro de áreas como teorias de unificação ${ }^{3}$.

A cromodinâmica quântica, teoria sobre a interação forte e que faz parte do modelo padrão, é um exemplo de teoria de calibre não abeliana, onde o grupo de calibre é o $S U(3)$. A carga forte é denominada de "cor", onde se faz uma analogia ${ }^{4}$ com as três cores bases vermelho, azul e verde. Já a eletrodinâmica clássica é um exemplo de teoria de calibre abeliana, onde o grupo de calibre é o $U(1)$ e temos dois tipos de cargas: positiva e negativa.

Se $g$ na Eq. (A.1) não dependesse do ponto do espaço-tempo teríamos um exemplo de transformação global. Essas transformações embora não sejam transformações de calibre são muito relevantes já que pelo Teorema de Noether [20] a invariância por tais transformações implica na conservação de quantidades físicas. Por exemplo, o número bariônico (a quantidade de quarks menos a quantidades de antiquarks) é conservado no modelo padrão devido a presença de uma simetria global relacionada com o grupo $U(1)$.

Na prática usamos a representação matricial de um grupo, isso é, os elementos de um grupo são representados por matrizes que respeitam a regra que define o grupo. Usualmente, os campos de matéria como $\Phi$ se transformam na representação fundamental do grupo de calibre. A

\footnotetext{
${ }^{2}$ Um grupo de Lie é um grupo contínuo que também é uma variedade diferenciável. Tais grupos são relevantes para física, especialmente a classe de grupos compactos na qual qualquer representação finita é equivalente a uma representação unitária [44]. Usualmente trabalhamos com representações matriciais que levam elementos de um grupo em uma matriz. Sendo assim, o produto que define o grupo passa a ser dado pelo o produto matricial e o elemento identidade é representado simplesmente pela matriz identidade do espaço linear considerado. Logo, grupos compactos levam à representações dadas por matrizes unitárias, sendo que a unitariedade é uma condição de extrema importância na física. Tome como exemplo: a matriz $S$, que deve ser unitária. Um exemplo em mecânica quântica é o propagador que dita a evolução temporal de um sistema que também deve ser unitário para conservar a norma dos estados. Nos dois casos temos que a unitariedade garante que a probabilidade seja conservada.

${ }^{3}$ Como a grande teoria de unificação de Georgi e Glashow que usa o $S U(5)$ [46].

${ }^{4}$ Ficando claro que a analogia não passa de uma convenção devido o número de cargas para a força forte (mediada pelos glúon) ser igual a quantidade de cores bases.
} 
representação fundamental de um elemento $g \in G(N)$, quando $G(N)$ é um grupo de matrizes $N \times N$, é ele mesmo (a própria matriz $N \times N$ ), $R_{\text {fun }}[g]=g$ e o campo de matéria $\Phi$ é então representado por uma matriz coluna $N \times 1$. Sendo assim, uma transformação de calibre pode ser representada genericamente por:

$$
\Phi_{i}^{\prime}=R[g]_{i j}\left(x^{\mu}\right) \Phi_{j}
$$

onde $R[g]$ é a representação do elemento $g$ numa representação qualquer $R$.

\section{A.2 Derivada covariante e o campo de calibre}

Se a ação de uma teoria é invariante sobre a transformação de calibre acima dizemos que a teoria é uma teoria de calibre sobre o grupo $G$. Contundo campos usualmente possuem termos dinâmicos que dependem da sua derivada e a derivada de um campo não é covariante (não se transforma da mesma forma que o campo) em relação a transformação de calibre da Eq. (A.1) como vemos abaixo:

$$
\partial_{\mu} \Phi^{\prime}=\left(\partial_{\mu} g\right) \Phi+g \partial_{\mu} \Phi
$$

Para contornar esse problema e assim obter ações que são invariantes sobre transformações de calibre, definimos a derivada covariante ${ }^{5}$

$$
D_{\mu} \Phi=\partial_{\mu}-i e A_{\mu} \Phi
$$

onde introduzimos o campo $A_{\mu}$, conhecido como o campo de calibre. A introdução de tal termo nas derivadas é o famoso princípio de acoplamento mínimo. Sendo o mesmo princípio considerado quando trabalhamos com a formulação hamiltoniana da eletrodinâmica clássica e fazemos a substituição $p_{\mu} \rightarrow p_{\mu}-e A_{\mu}$.

Usando $D_{\mu} \Phi^{\prime}=g D_{\mu} \Phi$ mostra-se que o campo de calibre se transforma na representação adjunta $^{6}$ associada ao grupo de calibre $G$. Essa transformação é dada por

$$
A_{\mu}^{\prime}=g A_{\mu} g^{-1}-\frac{i}{e}\left(\partial_{\mu} g\right) g^{-1}
$$

\section{A.3 Teoria de Yang-Mills pura}

Vemos pela definição da derivada covariante que de certa forma o campo de calibre $A_{\mu}$ é uma generalização do potencial vetor da eletrodinâmica clássica. Sendo assim podemos definir também

\footnotetext{
${ }^{5}$ Usamos a constante de acoplamento $e$ em vez de $g$ para não confundir com um elemento do grupo $G$.

${ }^{6} \mathrm{~A}$ representação adjunta é induzida pela álgebra do grupo de Lie, onde $T^{a} R_{\mathrm{adj}}^{a b}[g]=g T^{b} g^{-1}$, sendo $R_{\text {adj }}[g]$ a matriz que representa $g$ na representação adjunta. A álgebra de lie $\mathfrak{g}$ associada ao grupo de Lie $G$ é essencialmente a parte local do grupo. Tais álgebras de Lie são espaços lineares (espaço tangente do grupo de Lie) munidos do comutador de Lie [,] (numa representação matricial $[A, B]=A B-B A$, isso é, o comutador de Lie é o comutador usual). A base dessas álgebras são dadas pelos chamados geradores $T^{a}$ que satisfazem a condição de fechamento: $\left[T^{a}, T^{b}\right]=i f^{a b}{ }_{c} T^{c}$, onde $f^{a b}{ }_{c}$ (para grupos de Lie compactos que consideraremos, $f^{a b c}=f^{a b}{ }_{c}$ ) são as chamadas constantes de estrutura da álgebra. Uma álgebra de Lie é descrita completamente por essas constantes. Além de formarem uma base para a álgebra também geram os elementos do grupo através do mapa exponencial, basicamente $g=e^{T^{a} \theta_{a}}=1+T^{a} \theta_{a}+\cdots$.
} 
o tensor de intensidade de campo $F_{\mu \nu}$ através da derivada covariante por:

$$
F_{\mu \nu}=+\frac{i}{e}\left[D_{\mu}, D_{\nu}\right]=\partial_{\mu} A_{\nu}-\partial_{\nu} A_{\mu}-i e\left[A_{\mu}, A_{\nu}\right]
$$

Se o grupo de Lie é abeliano, os geradores do grupo comutam, tal que, o tensor de intensidade de campo se reduz a

$$
F_{\mu \nu}=\partial_{\mu} A_{\nu}-\partial_{\nu} A_{\mu}
$$

o mesmo tensor encontrado na eletrodinâmica clássica. Seguindo essa analogia, podemos definir a lagrangiana [2]

$$
\mathcal{L}_{\mathrm{YM}}=-\frac{1}{2} \operatorname{Tr}\left(F^{\mu \nu} F_{\mu \nu}\right),
$$

onde consideramos o tensor de intensidade de campo dado na Eq. (A.6) para um grupo de lie compacto $G .^{7}$ Essa teoria é conhecida como Teoria de YM pura (com grupo de calibre $G$ ).

Para obter a forma usual (formulação de segunda ordem) da Lagrangiana da teoria de YM pura dada na Eq. (2.1) precisamos considerar o campo de calibre na representação adjunta. Sabemos que o campo de calibre é um elemento da álgebra já que se transforma na representação adjunta, logo podemos escrevê-lo como:

$$
A_{\mu}=A_{\mu}^{a} T^{a}
$$

onde $A_{\mu}^{a}$ são as componentes ${ }^{8}$ do campo de calibre dentro da álgebra. Para eliminar os geradores usamos a relação de ortogonalidade:

$$
\operatorname{Tr}\left(T^{a} T^{b}\right)=\frac{1}{2} \delta^{a b}
$$

Usando as Eqs. (A.9) e (A.10) na Eq. (A.6) obtemos as componentes do tensor intensidade de campo

$$
F_{\mu \nu}^{a}=\partial_{\mu} A_{\nu}^{a}-\partial_{\nu} A_{\mu}^{a}+e f^{a b c} A_{\mu}^{b} A_{\nu}^{c}
$$

Logo, pela Eq. (A.8):

$$
\begin{aligned}
\mathcal{L}_{\mathrm{YM}} & =-\frac{1}{2} F_{\mu \nu}^{a} \operatorname{Tr}\left(T^{a} T^{b}\right) F^{b \mu \nu} \\
& =-\frac{1}{4} F_{\mu \nu}^{a} F^{a \mu \nu}
\end{aligned}
$$

resultando na Lagrangiana da teoria de YM pura:

$$
\mathcal{L}_{\mathrm{YM}}=-\frac{1}{4}\left(\partial_{\mu} A_{\nu}^{a}-\partial_{\nu} A_{\mu}^{a}+e f^{a b c} A_{\mu}^{b} A_{\nu}^{c}\right)^{2} .
$$

\footnotetext{
${ }^{7}$ Precisamos considerar um grupo compacto para garantir que o traço Tr seja bem definido fazendo o papel de produto interno.

${ }^{8}$ Existe uma ambiguidade na definição do campo de calibre. Podemos ver tanto $A_{\mu}^{a}$ quanto $A_{\mu}$ como o campo de calibre. No ponto de vista geométrico a escolha de $A_{\mu}$ é mais relevante já que não depende de uma base. Porém, em física de partículas, o uso das componentes $A_{\mu}^{a}$ como o campo de calibre é mais tradicional já que o campo $A_{\mu}^{a}$ tem um papel mais relevante, na prática (como computar as correções quânticas) precisamos de uma base de geradores.
} 


\section{APÊNDICE B}

\section{Funcionais Geradores}

Neste apêndice introduzimos rapidamente o formalismo de integrais de trajetória usando o conceito de funcionais geradores. Trabalharemos com um campo escalar $\phi$ com uma ação genérica dada por

$$
S=\int d^{d} x\left(\frac{1}{2} \phi D \phi+\mathcal{L}_{\text {int }}\right)
$$

onde $D$ é um operador diferencial que a princípio só precisa ser inversível e $\mathcal{L}_{\text {int }}$ são termos de interação (com três ou mais campos).

Se fossemos considerar apenas teorias físicas o operador $D$ precisaria ser ao menos positivo definido. Porém, para nossa análise podemos ignorar esses detalhes e considerar simplesmente que $D$ possui uma inversa. Como é amplamente conhecido para o caso de teorias de calibre não abelianas precisamos do procedimento de Faddeev-Popov para obter um termo inversível; isso se dá pela adição de termos na lagrangiana relativos a fixação de calibre e aos campos fictícios (fantasmas) responsáveis em garantir a unitariedade da teoria [20].

\section{B.1 Funcional gerador $Z$}

Para uma ação como da Eq. (B.1) podemos imediatamente seguir com a sua quantização através da integral de trajetória

$$
Z[J]=\frac{1}{Z[0]} \int \mathcal{D} \phi \exp i\left(S+\int d^{d} x J(x) \phi(x)\right)
$$

onde consideramos a introdução de uma fonte ${ }^{1} J$ para o campo $\phi$. O funcional gerador pela definição Eq. (B.2) pode ser normalizado, tal que,

$$
Z[0] \equiv \int \mathcal{D} \phi \exp i S=1 .
$$

Tal normalização desconsidera as bolhas de vácuo que contribuem com $Z[0]$.

\footnotetext{
${ }^{1}$ Essa é um procedimento usual baseado no trabalho de Schwinger que apareceu inicialmente no artigo [14].
} 
Bolhas de vácuo são diagramas que não tem linha externas. Esse tipo de diagrama usualmente não é interessante em teoria quântica de campos, porém, por exemplo, para obter a densidade de energia do vácuo como no caso do Efeito Casimir e em cosmologia [47] são relevantes. Além disso, as bolhas de vácuo são importante no formalismo de teoria de campos à temperatura finita [48], onde diagramas como os da Fig. 6.3 [49, Fig. 6] representariam contribuições perturbativas para diversas quantidades físicas, tais como pressão e energia livre.

A fonte $J$ é um artifício que nos permite gerar as funções de Green a partir do funcional gerador $Z[J]$. Para vemos isso considere a derivada funcional da Eq. (B.2) em relação a fonte $J$ :

$$
\frac{\delta Z[J]}{\delta J(x)}=\int \mathcal{D} \phi i \phi(x) \exp i\left(S+\int d^{d} x J(x) \phi(x)\right) \equiv i\langle 0|\phi(x)| 0\rangle_{J},
$$

onde $\langle\phi\rangle_{J}$ é a correlação do campo $\phi$ com a presença da fonte $J$. Fazendo $J=0$, temos que

$$
\left.\frac{1}{i} \frac{\delta Z[J]}{\delta J(x)}\right|_{J=0}=\int \mathcal{D} \phi \phi(x) \exp i S=\langle 0|\phi(x)| 0\rangle
$$

isso é, obtemos o valor esperado no vácuo do campo $\phi$.

Generalizando o resultado acima obtemos as funções de Green $n$-pontos

$$
\begin{aligned}
G^{(n)}\left(x_{1}, \ldots, x_{n}\right) & =\left.\frac{1}{i^{n}} \frac{\delta^{n} Z[J]}{\delta J\left(x_{1}\right) \cdots \delta J\left(x_{n}\right)}\right|_{J=0} \\
& =\int \mathcal{D} \phi \phi\left(x_{1}\right) \cdots \phi\left(x_{n}\right) \exp i S=\left\langle 0\left|T \phi\left(x_{1}\right) \cdots \phi\left(x_{n}\right)\right| 0\right\rangle
\end{aligned}
$$

Vale mencionar que o operador de ordenação de tempo

$$
T \phi\left(t_{1}\right) \phi\left(t_{2}\right)=\phi\left(t_{1}\right) \phi\left(t_{2}\right) \theta\left(t_{1}-t_{2}\right)+\phi\left(t_{2}\right) \phi\left(t_{1}\right) \theta\left(t_{2}-t_{1}\right)
$$

onde $\theta(t)$ é a função degrau de Heaviside, é necessário uma vez que o campo nas integrais de trajetória não são mais operadores e comutam entre si como $c$-numbers. Os detalhes podem ser encontrados em [22].

O funcional gerador $Z$ da Eq. (B.2) pode ser computado perturbativamente gerando tanto diagramas conexos quanto desconexos. Note que, pela normalização as bolhas de vácuo não aparecem. Isso se dá pela fatoração entre a contribuição total devida as bolhas de vácuo e os demais diagramas. Geralmente estamos interessados nos diagramas conexos, pois são eles que contribuem para a matriz $S$. Além disso, como veremos adiante os diagramas desconexos podem ser obtidos através de uma classe mais elementar de diagramas conexos, os chamados diagramas 1PI (one particle irreducible).

\section{B.2 Funcional gerador $W$}

Podemos definir o funcional gerador dos diagramas conexos $W$ através do funcional gerador $Z$ por

$$
W[J]=-i \ln Z[J]
$$


Como exemplo considere a correlação de 2-pontos gerada por $W[J]$ :

$$
\begin{aligned}
\langle 0|T \phi(x) \phi(y)| 0\rangle_{\mathrm{C}, J} & =\frac{1}{i} \frac{\delta^{2} W[J]}{\delta J(x) \delta J(y)} \\
& =-i \frac{\delta}{\delta J(x)}\left(\frac{1}{Z[J]} \int \mathcal{D} \phi \phi(y) \exp i S\right) \\
& =\langle 0|T \phi(x) \phi(y)| 0\rangle_{J}-\langle 0|\phi(x)| 0\rangle_{J}\langle 0|\phi(y)| 0\rangle_{J} .
\end{aligned}
$$

Vemos que a correlação gerada é igual a correlação obtida com o gerador $Z[J]$ subtraindo a parte desconexa que no caso é simplesmente o produto das correlações do campo. Portanto para $J=0$, temos a função de Green conexa de 2-pontos:

$$
\begin{aligned}
\langle 0|T \phi(x) \phi(y)| 0\rangle_{\mathrm{C}} & =\langle 0|T \phi(x) \phi(y)| 0\rangle-\langle 0|\phi(x)| 0\rangle\langle 0|\phi(y)| 0\rangle \\
& =\left.\frac{1}{i} \frac{\delta^{2} W[J]}{\delta J(x) \delta J(y)}\right|_{J=0} .
\end{aligned}
$$

Porém, usualmente a função de Green de 1-ponto é tal que $\langle 0|T \phi(x)| 0\rangle=0 .^{2}$ A Eq. (B.10) mostra então que

$$
\langle 0|T \phi(x) \phi(y)| 0\rangle_{\mathrm{C}}=\langle 0|T \phi(x) \phi(y)| 0\rangle,
$$

ou seja, a função de Green conexa de 2-pontos é igual a função Green desconexa de 2-pontos. Contudo, ressaltamos que para além de 2-pontos elas geralmente não coincidem.

Generalizando, a função de Green conexa de $n$-pontos é gerada por $W[J]$, tal que

$$
\left\langle 0\left|T \phi\left(x_{1}\right) \cdots \phi\left(x_{n}\right)\right| 0\right\rangle_{\mathrm{C}}=(-i)^{n-1} \frac{\delta^{n} W[J]}{\delta J\left(x_{1}\right) \cdots \delta J\left(x_{n}\right)} .
$$

\section{B.3 Ação efetiva}

Note que a Eq. (B.4) pode ser reescrita com o gerador $W[J]$ como

$$
\phi_{J}(x) \equiv\langle 0|\phi(x)| 0\rangle_{J}=\frac{\delta W[J]}{\delta J}
$$

definindo o chamado campo clássico $\phi_{J}(x)$. A Eq. (B.13) indica que $J(x)$ e $\phi_{J}(x)$ formam um par conjugado em relação ao funcional gerador $W[J]$. Se a relação acima for inversível podemos fazer uma transformada de Legendre, tal que obtemos uma novo funcional em relação ao campo clássico:

$$
\Gamma\left[\phi_{J}\right]=W[J]-\int d^{d} x \phi_{J}(x) J(x) .
$$

O funcional gerador $\Gamma\left[\phi_{J}\right]$ é conhecido como ação efetiva sendo fácil ver uma razão para isso. Derivando a ação efetiva por $\phi_{J}(x)$ chegamos na expressão

$$
\frac{\delta \Gamma\left[\phi_{J}\right]}{\delta \phi_{J}(x)}=\int d^{d} x^{\prime} \frac{\delta \phi_{J}\left(x^{\prime}\right)}{\delta J(x)} \frac{\delta W[J]}{\delta J\left(x^{\prime}\right)}-J(x)-\int d^{d} x^{\prime} \phi_{J}\left(x^{\prime}\right) \frac{\delta J(x)}{\delta \phi_{J}\left(x^{\prime}\right)}
$$

\footnotetext{
${ }^{2}$ Caso contrário podemos fazer um shift no campo tal que a função de Green de 1-ponto se anule; um valor diferente de zero indica uma quebra espontânea de simetria [22, pag. 188].
} 
que pela definição do campo clássico dada na Eq. (B.13) se reduz a

$$
\frac{\delta \Gamma\left[\phi_{J}\right]}{\delta \phi_{J}(x)}=-J(x)
$$

Essa equação tem a mesma forma da equação de movimento dada a partir da ação $S$ pelas equações de Euler-Lagrange indicando que a ação efetiva é a versão quântica da ação $S$. Pode-se demonstrar $[22,50]$ que a ação efetiva $\Gamma\left[\phi_{J}\right]$ realmente se reduz a ação $S$ (com fontes) no limite $\hbar \rightarrow 0$. Isso é, as contribuições da ação efetiva ao nível árvore são iguais as contribuições clássicas devido a ação clássica [50].

A ação efetiva também pode ser vista como um gerador funcional. Conseguimos ver isso derivando a Eq. (B.16) novamente pelo campo clássico, tal que,

$$
\frac{\delta^{2} \Gamma\left[\phi_{J}\right]}{\delta \phi_{J}(y) \delta \phi_{J}(x)}=-\frac{\delta J(x)}{\delta \phi_{J}(y)}=-\left(\frac{\delta \phi_{J}(y)}{\delta J(x)}\right)^{-1} .
$$

Usando a definição (B.13) obtemos que

$$
\frac{\delta^{2} \Gamma\left[\phi_{J}\right]}{\delta \phi_{J}(y) \delta \phi_{J}(x)}=-\left(\frac{\delta^{2} W[J]}{\delta J(x) \delta J(y)}\right)^{-1} .
$$

Para $J=0,{ }^{3}$ substituindo a Eq. (B.10) na Eq. (B.17) concluímos que

$$
\left.\frac{\delta^{2} \Gamma\left[\phi_{J}\right]}{\delta \phi_{J}(y) \delta \phi_{J}(x)}\right|_{\phi_{J}=\phi_{0}}=i\langle 0|T \phi(x) \phi(y)| 0\rangle^{-1}
$$

Isso demostra que a segunda derivada da ação efetiva é proporcional a inversa do propagador completo $\langle 0|T \phi(x) \phi(y)| 0\rangle$.

Podemos reescrever a Eq. (B.18) de uma forma mais esclarecedora ${ }^{4}$ :

$$
\Gamma_{(2)}=-W_{(2)}^{-1} W_{(2)} W_{(2)}^{-1},
$$

onde usamos a notação

$$
\Gamma_{(n)}=\Gamma\left(x_{1}, \ldots, x_{n}\right) \equiv \frac{\delta^{n} \Gamma\left[\phi_{J}\right]}{\delta \phi_{J}\left(x_{1}\right) \cdots \delta \phi_{J}\left(x_{n}\right)}
$$

e

$$
W_{(n)}=W\left(x_{1}, \ldots, x_{n}\right) \equiv \frac{\delta^{n} W[J]}{\delta J\left(x_{1}\right) \cdots \delta J\left(x_{n}\right)} .
$$

A equação Eq. (B.20) indica que a ação efetiva gera diagramas com as pernas "amputadas" pela inversa dos propagadores completos.

Mostraremos que isso vale para $n>2$ e que os diagramas gerados são os importantes diagramas 1PI. Ressaltamos que para $n=2$ isso não é válido, já que a inversa do propagador inteiro não é considerado um diagrama 1PI. Sendo assim, para $n>2, \Gamma_{(n)}$ é chamado de função

\footnotetext{
${ }^{3}$ Para a ação efetiva a condição para o campo clássico é dada pela Eq. (B.16) com $J=0$. Logo, $\phi_{J}(x)=\phi_{0}$, onde $\phi_{0}$ é a constante que satisfaz $\left.\frac{\delta \Gamma\left[\phi_{J}\right]}{\delta \phi_{J}}\right|_{\phi_{J}(x)=\phi_{0}}=0$.

${ }^{4}$ Os índices e as integrais devido aos produtos podem ser omitidos usando a notação de DeWitt. Por exemplo, $W_{(2)} \cdot \Gamma_{(2)} \equiv \int d^{d} z W(x, z) \Gamma(z, y)$.
} 


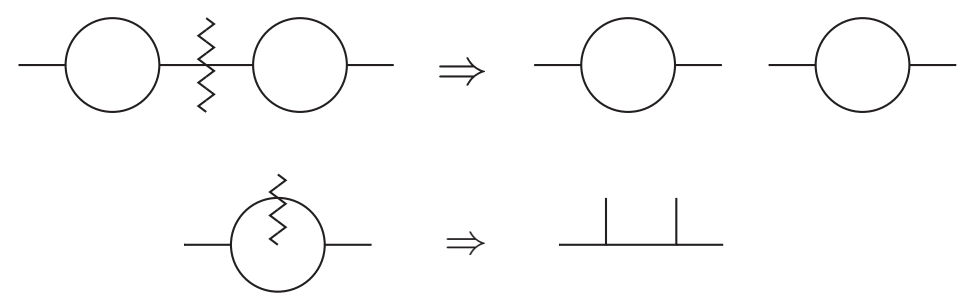

Figura B.1: O primeiro diagrama não é um diagrama 1PI, pois ao cortar a linha interna (marcada com a linha em ziguezague) separamos o diagrama em dois. Enquanto, para o segundo diagrama, o corte de qualquer linha interna leva a um diagrama conexo.

de vértice próprio de $n$-pontos, enquanto para $n=2$ (de acordo com a Eq. (B.20)) é somente a inversa do propagador inteiro.

Mais uma vez podemos derivar a Eq. (B.17) pelo campo clássico:

$$
\begin{aligned}
\frac{\delta^{3} \Gamma\left[\phi_{J}\right]}{\delta \phi_{J}(z) \delta \phi_{J}(y) \delta \phi_{J}(x)} & =-\frac{\delta}{\delta \phi_{J}(z)}\left(\frac{\delta \phi_{J}(y)}{\delta J(x)}\right)^{-1} \\
& =\int d^{d} x^{\prime} d^{d} y^{\prime}\left[\left(\frac{\delta \phi_{J}(x)}{\delta J\left(x^{\prime}\right)}\right)^{-1}\left(\frac{\delta \phi_{J}(y)}{\delta J\left(y^{\prime}\right)}\right)^{-1}\right] \frac{\delta}{\delta \phi_{J}(z)}\left(\frac{\delta \phi_{J}(y)}{\delta J(x)}\right) \\
& =W^{-1}\left(x, x^{\prime}\right) \cdot W^{-1}\left(y, y^{\prime}\right) \int d^{d} z^{\prime} \frac{\delta J\left(z^{\prime}\right)}{\delta \phi_{J}(z)} \frac{\delta}{\delta J\left(z^{\prime}\right)}\left(\frac{\delta \phi_{J}(y)}{\delta J(x)}\right) \\
& =W^{-1}\left(x, x^{\prime}\right) \cdot W^{-1} \cdot\left(y, y^{\prime}\right) \int d^{d} z^{\prime}\left(\frac{\delta \phi_{J}(z)}{\delta J\left(z^{\prime}\right)}\right)^{-1}\left(\frac{\delta^{3} W[J]}{\delta J\left(z^{\prime}\right) \delta J\left(x^{\prime}\right) \delta J\left(y^{\prime}\right)}\right) \\
& =W^{-1}\left(x, x^{\prime}\right) \cdot W^{-1}\left(y, y^{\prime}\right) \cdot W^{-1}\left(z, z^{\prime}\right) \cdot \frac{\delta^{3} W[J]}{\delta J\left(x^{\prime}\right) \delta J\left(y^{\prime}\right) \delta J\left(z^{\prime}\right)}
\end{aligned}
$$

demostrando que a derivada terceira da ação efetiva em relação ao campo clássico é igual a função de Green conexa de 3-pontos com as pernas amputadas:

$$
\Gamma_{(3)}=W_{(2)}^{-1} W_{(2)}^{-1} W_{(2)}^{-1} W_{(3)}
$$

Com $J=0$, a equação acima mostra que $\left.\Gamma_{(3)}\right|_{\phi_{J}=\phi_{0}}$ é igual a função de vértice próprio de 3 -pontos.

Isso pode ser generalizado para $n$-pontos, tal que, as funções de Green conexas de $n$-pontos com pernas amputadas para $n>2$, denotada por $\Gamma_{0}\left(x_{1}, \ldots, x_{n}\right)$, são geradas pela ação efetiva:

$$
\Gamma_{0}\left(x_{1}, \ldots, x_{n}\right)=\left.\frac{\delta^{n} \Gamma\left[\phi_{J}\right]}{\delta \phi_{J}\left(x_{1}\right) \cdots \delta \phi_{J}\left(x_{n}\right)}\right|_{\phi_{J}=\phi_{0}} .
$$

Pode-se mostrar [51, Section 16.1] que tais funções de Green conexas com pernas amputadas são iguais a soma de todos os diagramas 1PI, isso é, a função de vértice próprio de $n$-pontos.

Uma diagrama é dito $1 P I$ se ao "cortar" qualquer perna interna ele ainda continua conexo. Na Fig. B.1, temos um exemplo de diagrama não 1PI que se reduz a dois diagramas 1PI ao cortar uma das suas pernas internas. Os diagramas 1PI são definidos com suas pernas amputadas, tal que, a ação efetiva $\Gamma\left[\phi_{J}\right]$ é dita o funcional gerador dos diagramas 1PI. 
Tais diagramas são interessantes já que podemos reescrever uma expansão perturbativa em termo do número de loops. Tomando o caso do propagador, pela Eq. (B.20), podemos escrever:

$$
\Gamma(x, y)=i G^{-1}(x, y)=i G_{0}^{-1}(x, y)-i \Pi(x, y)
$$

onde $G_{0}(x, y)$ é o propagador livre e $\Pi(x, y)$ é conhecido como a autoenergia. A autoenergia é a soma de todos os diagramas 1PI com 2 pernas externas (amputadas), ou seja, pela nossa notação $\Pi(x, y)=\Gamma_{0}(x, y)$. Note que $\Gamma_{0}(x, y)$ não é definido pela Eq. (B.25) (já que não é definida para $n=2$, mas sim pela Eq. (B.26)).

A Eq. (B.26) pode ser reescrita como (omitido os argumentos por clareza)

$$
G=\left[G_{0}^{-1}-\Pi\right]^{-1}=G_{0}+G_{0} \Pi G_{0}+G_{0} \Pi G_{0} \Pi G_{0}+\cdots .
$$

Isso é, uma expansão em série de potências da autoenergia П. Usualmente a autoenergia é dada por diagramas de um loop, mostrando que o propagador inteiro $G$ pode ser dado por uma expansão perturbativa em termos de loops. Em ordem mais baixa (nível árvore) teríamos o propagador livre $G_{0}\left(\right.$ ordem $\hbar^{0}$ ), na ordem superior $G_{0} \Pi G_{0}$, geralmente [52], um termo em ordem $\hbar$ e assim em diante. Logo, a série em termo de loops equivale, geralmente, a uma série de potência em $\hbar$, ou seja: uma série de correções quânticas.

Na tabela B.1 temos exemplos de contribuições (na forma diagramática) para cada funcional gerador mencionado nesse apêndice.

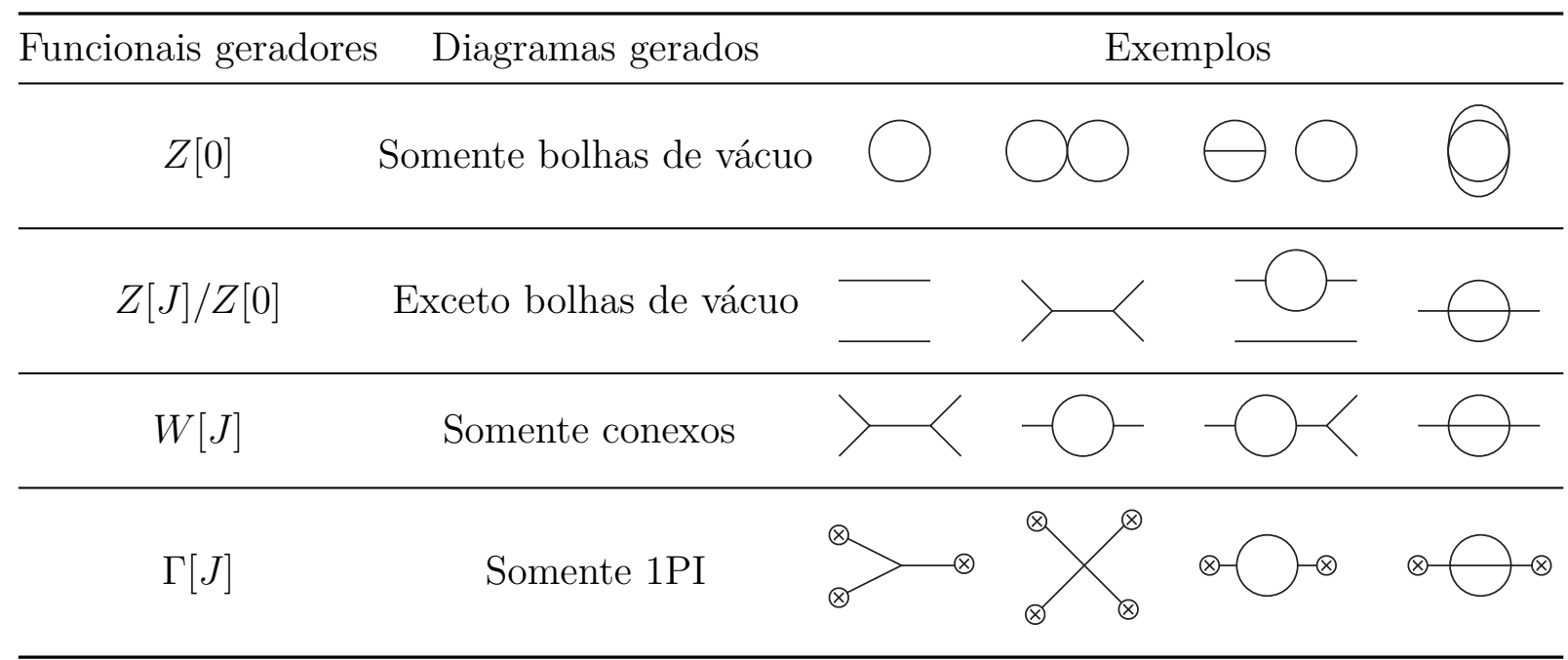

Tabela B.1: Funcionais geradores e exemplos de diagramas de Feynman gerados perturbativamente. Note que os diagramas 1PI têm suas pernas amputadas. 


\section{APÊNDICE C}

\section{REGRAS DE FEYNMAN}

Aqui deduziremos as conhecidas Regras de Feynman para o formalismo de segunda ordem da teoria de YM. Mostraremos também as convenções usadas que concordam com Peskin \& Schroeder [21]. Além disso, obteremos mais concisamente as regras para gravitação.

Para obter as regras de Feynman utilizaremos a mesma abordagem encontrada em [9, Apêndice A] que baseia-se no fato que o gerador das funções 1PI, a ação efetiva $\Gamma$, é igual a ação $S$ para $\hbar \rightarrow 0$. No regime clássico podemos obter as regras de Feyman uma vez que os efeitos quânticos são associados a loops. ${ }^{1}$

\section{C.1 Propagador}

O propagador pode ser obtido através da expressão dada na Eq. (B.19) ${ }^{2}$ no limite $\hbar \rightarrow 0$ :

$$
\frac{\delta^{2} S^{(2)}}{\delta \phi(y) \delta \phi(x)}=i\langle\phi(x) \phi(y)\rangle^{-1}
$$

onde $S^{(2)}$ é a parte bilinear nos campos da ação $S, \phi_{J}(x) \rightarrow \phi(x)$ e o propagador inteiro se reduz ao propagador livre: $\langle 0|T \phi(x) \phi(y)| 0\rangle \rightarrow\langle\phi(x) \phi(y)\rangle$. Da Eq. (C.1) obtemos o propagador livre em termo de $i S$ :

$$
\langle\phi(x) \phi(y)\rangle=-\left(\frac{\delta^{2} i S^{(2)}}{\delta \phi(x) \delta \phi(y)}\right)^{-1} .
$$

Podemos usar a expressão ${ }^{3}$ acima para obter a Regra de Feynman para o propagador (livre) no espaço da posição.

\footnotetext{
${ }^{1}$ Embora usualmente se considere que o contrário também seja verdade, isso é, contribuições com um ou mais loops sejam quânticas conforme [52] isso não é geralmente válido.

${ }^{2}$ Restringindo para os termos bilineares nos campos da ação não precisamos tomar a derivada com os campos se anulando.

${ }^{3}$ Ela é válida a princípio para campos bosônicos, mas pode ser facilmente estendida para campos de carácter fermiônico como os campos fantasmas $c, \bar{c}$.
} 
Para obter o propagador no espaço dos momentos usamos a transformação de Fourier que é definida aqui por:

$$
f(x)=\int \frac{d^{d} p}{(2 \pi)^{d}} \tilde{f}(p) e^{-i p x} \quad \text { e } \quad \tilde{f}(p)=\int d^{d} x f(x) e^{i p x}
$$

onde $p x \equiv p_{0} t-\mathbf{p} \cdot \mathbf{x} .{ }^{4}$ Com a função delta sendo definida de acordo com as mesmas convenções por

$$
\delta(x-y)=\int \frac{d^{d} p}{(2 \pi)^{d}} e^{-i p(x-y)} \quad \text { e } \quad(2 \pi)^{d} \delta(p)=\int d^{d} x e^{i p x} .
$$

Assim o propagador livre no espaço dos momentos é dado por

$$
\langle\phi(p) \phi(q)\rangle=\int d^{d} x d^{d} y\langle\phi(x) \phi(y)\rangle e^{i p x} e^{i q y} .
$$

Podemos simplificar a expressão acima fazendo $y=x+z$ e usando que a função de Green de 2-pontos somente depende do intervalo $y-x$. Pela definição da função delta dada na Eq. (C.4) teríamos que

$$
\begin{aligned}
\langle\phi(p) \phi(q)\rangle & =\int d^{d} x d^{d} z\langle\phi(0) \phi(z)\rangle e^{i(p+q) x} e^{i q z} \\
& =(2 \pi)^{d} \delta(p+q) \int d^{d} z\langle\phi(0) \phi(z)\rangle e^{i q z} .
\end{aligned}
$$

Ou seja, no espaço dos momentos, a dependência no intervalo $y-x$ é igual a considerar a conservação de momento, tal que podemos considerar que $p=-q$ na Eq. (C.5):

$$
\langle\phi(-q) \phi(q)\rangle=\int d^{d} x d^{d} y\langle\phi(x) \phi(y)\rangle e^{i q(y-x)},
$$

onde $y-x \equiv z$. A expressão acima é suficiente, mas usualmente podemos inferir o propagador no espaço do momento pela inversa:

$$
\langle\phi(x) \phi(y)\rangle=\int \frac{d^{d} p}{(2 \pi)^{d}}\langle\phi(-p) \phi(p)\rangle e^{-i p(y-x)} .
$$

\section{C.2 Vértices}

Para obter os vértices da teoria lembramos que a ação efetiva gera os vértices próprios. Sendo assim a Eq. (B.25) no limite $\hbar \rightarrow 0$ é igual a

$$
\Gamma_{0}\left(x_{1}, \ldots, x_{n}\right)=\left.\frac{\delta^{n} i S}{\delta \phi\left(x_{1}\right) \cdots \delta \phi\left(x_{n}\right)}\right|_{\phi=0},
$$

tal que, os vértices são dados pela soma de todas as permutações possíveis do termo acima:

$$
V_{\text {tree }}\left(x_{1}, \ldots, x_{n}\right)=\sum_{\sigma} \Gamma_{0}\left(\sigma\left(x_{1}, \ldots, x_{n}\right)\right)
$$

onde $\sigma$ permuta todas as pernas externas.

${ }^{4}$ Note que aqui estamos em $d$ dimensões $\left(d-1\right.$ espaciais e uma temporal), tal que $\mathbf{p} \cdot \mathbf{x}=\sum_{i=1}^{d-1} p_{i} x_{i}$. A função delta em $d$ dimensões $\delta^{(d)}(x-y)$ é denotada simplesmente por $\delta(x-y)$. 
No espaço do momento, os vértices são dados por

$$
V_{\text {tree }}\left(p_{1}, \ldots, p_{n}\right)=(2 \pi)^{d \cdot n} \frac{\delta^{n} i S}{\delta \tilde{\phi}\left(-p_{1}\right) \cdots \delta \tilde{\phi}\left(-p_{n}\right)}+\text { perm. }
$$

onde $S$ é escrito no espaço dos momento e o termo perm. representa todas as permutações do primeiro termo. Na expressão acima os momentos estão entrando nos vértices externos $x_{1} \ldots x_{n}$. Isso é o oposto da nossa convenção que considera os momentos entrando no vértice interno (de interação).

Logo para obter os vértices, de acordo com a nossa convenção, a expressão adequada é obtida fazendo $p_{i} \rightarrow-p_{i}$ na Eq. (C.11) resultando na expressão

$$
V_{\text {tree }}\left(p_{1}, \ldots, p_{n}\right)=(2 \pi)^{d \cdot n} \frac{\delta^{n} i S}{\delta \tilde{\phi}\left(p_{1}\right) \cdots \delta \tilde{\phi}\left(p_{n}\right)}+\text { permutações. }
$$

\section{C.3 Teoria de Yang-Mills}

Nessa seção vamos deduzir em detalhes as regras de Feynman para a teoria de YM nos formalismos de segunda ordem, primeira ordem e diagonal usando a abordagem discutida na seção anterior.

\section{C.3.1 Formalismo de segunda ordem}

Pela Eq. (C.2) temos que o propagador livre do glúon pode ser obtido pela inversa do termo bilinear no campo de calibre $A_{\mu}^{a}$ :

$$
P_{\mu \nu}^{a b}=-\left(\frac{\delta^{2} i S_{\mathrm{eff}}^{(2)}}{\delta A^{a \mu}(x) \delta A^{b \nu}(y)}\right)^{-1},
$$

usando a ação efetiva da teoria de YM no formalismo de segunda ordem definida por

$$
S_{\text {eff }}=\int d^{d} x \mathcal{L}_{\text {eff }}^{(2)}
$$

A Eq. (C.13) resulta em

$$
P_{\mu \nu}^{a b}(x-y)=\delta^{a b} \frac{i}{\partial^{2}}\left(\eta_{\mu \nu}+(\xi-1) \frac{\partial_{\mu} \partial_{\nu}}{\partial^{2}}\right) \delta(x-y) .
$$

Fazendo a transformada de Fourier (C.4) no propagador Eq. (C.15) a expressão pode ser escrita como

$$
P_{\mu \nu}^{a b}(x-y)=-i \delta^{a b} \int \frac{d^{d} p}{(2 \pi)^{d}} P_{\mu \nu}(p) e^{-i p(x-y)},
$$

onde podemos inferir o conhecido propagador do glúon

$$
P_{\mu \nu}^{a b}(p) \equiv-i \delta^{a b} P_{\mu \nu}(p)=-\frac{i}{p^{2}}\left(\eta_{\mu \nu}-(1-\xi) \frac{p_{\mu} p_{\nu}}{p^{2}}\right) \delta^{a b} .
$$

O propagador do campo fantasma pode ser encontrado de uma forma semelhante. Assim, temos as seguintes regras de Feynman para os propagadores na forma diagramática: 


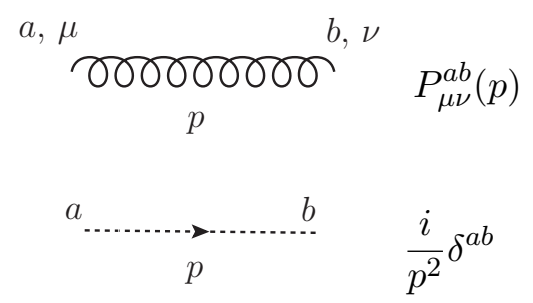

Para finalizar vamos mostrar como obter o vértice cúbico usando a Eq. (C.12) que nesse caso pode ser escrita como

$$
V_{\mu \nu \rho}^{a b c}(k, p, q)=\left.(2 \pi)^{3 d} \frac{\delta^{3} i S_{\mathrm{eff}}^{(2)}}{\delta \tilde{A}^{a \mu}(k) \delta \tilde{A}^{b \nu}(p) \delta \tilde{A}^{c \rho}(q)}\right|_{A=0}+\text { perm. }
$$

onde já estamos no espaço do momento (incluindo a ação efetiva).

Considerando somente a parte que contribui, isso é, a parte cúbica da ação:

$$
S_{A A A}=-i g f^{a b c} \frac{1}{(2 \pi)^{3 d}} \int d^{d} x d^{d} k d^{d} p d^{d} q e^{-i(k+p+k) x}\left(-i k_{\nu} \eta_{\mu \rho}\right) \tilde{A}^{a \mu}(k) \tilde{A}^{b \nu}(p) \tilde{A}^{c \rho}(q),
$$

podemos integrar sobre $x$ obtendo uma função delta que assegurará a conservação de momento:

$$
S_{A A A}=-i g f^{a b c}(2 \pi)^{-2 d} \int d^{d} k d^{d} p d^{d} q \delta(k+p+q)\left(-i k_{\nu} \eta_{\mu \rho}\right) \tilde{A}^{a \mu}(k) \tilde{A}^{b \nu}(p) \tilde{A}^{c \rho}(q) .
$$

A base do vértice é dada então pela derivada da integral acima, conforme Eq. (C.12),

$$
(2 \pi)^{3 d} \frac{\delta^{3} i S_{A A A}}{\delta \tilde{A}^{a \mu}(k) \delta \tilde{A}^{b \nu}(p) \delta \tilde{A}^{c \rho}(q)}=-g f^{a b c} k_{\nu} \eta_{\mu \rho}(2 \pi)^{d} \delta(k+p+q) .
$$

Fazendo todas as permutações obtemos finalmente o vértice ${ }^{5}$

$$
V_{\mu \nu \rho}^{a b c}(k, p, q)=g f^{a b c}\left[\eta_{\mu \nu}(k-p)_{\rho}+\eta_{\nu \rho}(p-q)_{\mu}+\eta_{\rho \mu}(q-k)_{\nu}\right]
$$

de acordo com a Ref. [21]. O vértice $(A A A A)$ podem ser obtido de forma análoga.

Também mostraremos o vértice com os campos fantasmas. Considerando o termo do acoplamento do campo fantasma e o campo de calibre:

$$
S_{\bar{c} A c}=i g f^{a b c} \frac{1}{(2 \pi)^{3 d}} \int d^{d} x d^{d} k d^{d} p d^{d} q e^{-i(k+p+k) x}\left(-i p_{\mu}\right) \tilde{\bar{c}}^{a}(p) \tilde{A}^{b \mu}(k) \tilde{c}^{c}(q)
$$

e integrando sobre $x$ temos a função delta como anteriormente, obtemos que

$$
S_{\bar{c} A c}=i g f^{a b c} \frac{1}{(2 \pi)^{2 d}} \int d^{d} k d^{d} p d^{d} q \delta(k+p+q)\left(-i p_{\mu}\right) \tilde{\bar{c}}^{a}(p) \tilde{A}^{b \mu}(k) \tilde{c}^{c}(q) .
$$

$\mathrm{O}$ vértice pode ser derivado diretamente por

$$
(2 \pi)^{3 d} \frac{\delta^{3} i S_{\bar{c} A c}}{\delta \tilde{\bar{c}}^{a}(k) \delta \tilde{A}^{b \nu}(p) \delta \tilde{c}^{c}(q)}=g f^{a b c}(2 \pi)^{d} p_{\mu} \delta(p+k+q)
$$

Considerando nossa convenção, a regra de Feynman para o vértice do campo fantasma é

$$
V_{\mu}^{a b c}(p)=g f^{a b c} p_{\mu}
$$

\footnotetext{
${ }^{5} \mathrm{O}$ fator de $(2 \pi)^{d}$ e o delta de conservação serão omitidos. Sendo assim, devemos assumir a conservação de momento em cada vértice e definir a matriz $S$ com um fator geral $(2 \pi)^{d} \delta\left(P_{i}-P_{f}\right)$, onde $P_{i}$ é a soma do momento inicial e $P_{f}$ do momento final.
} 
também de acordo $^{6}$ com a Ref. [21, Apêndice A, eq. (A.13)].

As Regras de Feynman para os vértices da teoria de YM no formalismo de segunda ordem são dadas diagramaticamente (todos momentos estão entrando) por:

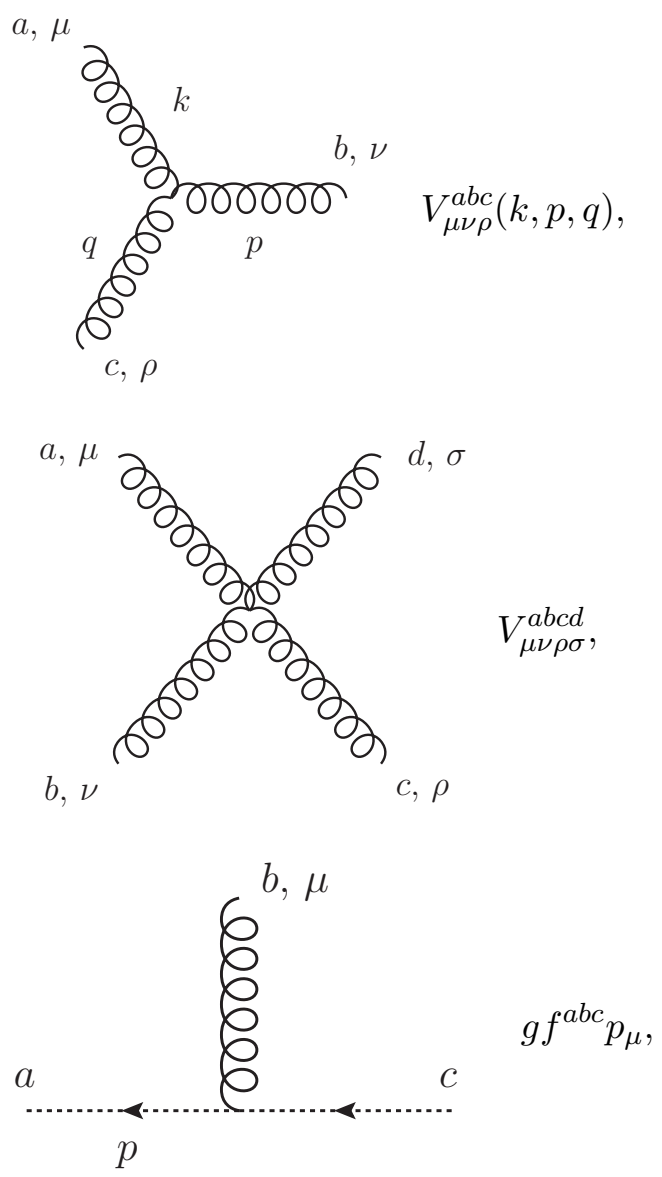

onde

$$
\begin{aligned}
V_{\mu \nu \rho \sigma}^{a b c d} \equiv- & i g^{2}\left[f^{a b e} f^{c d e}\left(\eta_{\mu \rho} \eta_{\nu \sigma}-\eta_{\mu \sigma} \eta_{\nu \rho}\right)\right. \\
+ & f^{a c e} f^{b d e}\left(\eta_{\mu \nu} \eta_{\rho \sigma}-\eta_{\mu \sigma} \eta_{\rho \nu}\right) \\
+ & \left.f^{a d e} f^{b c e}\left(\eta^{\mu \nu} \eta^{\rho \sigma}-\eta_{\mu \rho} \eta_{\nu \sigma}\right)\right]
\end{aligned}
$$

as molas representam o campo de calibre $A_{\mu}^{a}$ e as linhas pontilhadas representam os campos fantasmas.

\section{C.3.2 Formalismo de primeira ordem}

No caso do formalismo de primeira ordem temos um acoplamento dos campos no termo bilinear, sendo assim é necessário considerar a ação efetiva de primeira ordem escrita na forma matricial. O termo bilinear nos campos é dado por

$$
S_{\text {bilinear }}^{(1)}=\frac{1}{2} \int d^{d} x d^{d} y\left[A^{a \mu}(x) \quad F^{a \alpha \beta}(x)\right] M_{\mu \alpha \beta \nu \lambda \sigma}^{a} \stackrel{b}{b}(x-y)\left[\begin{array}{c}
A^{b \nu}(y) \\
F^{b \lambda \sigma}(y)
\end{array}\right],
$$

${ }^{6}$ Em Peskin \& Schroeder [21] no vértice do campo fantasma os momentos estão saindo. Aqui continuamos com a convenção que o momento está entrando no vértice, logo chegamos num resultado com sinal oposto. 
onde

$$
M_{\mu \alpha \beta \nu \lambda \sigma}^{a} \stackrel{b}{b}(x-y)=\delta^{a b} \delta(x-y)\left(\begin{array}{cc}
A_{\mu \nu} & B_{\mu \lambda \sigma} \\
C_{\alpha \beta \nu} & D_{\alpha \beta \lambda \sigma}
\end{array}\right)
$$

com

$$
\begin{gathered}
A_{\mu \nu}=-\frac{1}{\xi} \partial_{\mu} \partial_{\nu} \\
B_{\mu \lambda \sigma}=-\frac{\partial_{\lambda} \eta_{\sigma \mu}-\partial_{\sigma} \eta_{\lambda \mu}}{2}, \\
C_{\alpha \beta \nu}=\frac{\partial_{\alpha} \eta_{\beta \nu}-\partial_{\beta} \eta_{\alpha \nu}}{2}, \\
D_{\alpha \beta \lambda \sigma}=\frac{1}{2} I_{\alpha \beta \lambda \sigma} \equiv \frac{\eta_{\alpha \lambda} \eta_{\beta \sigma}-\eta_{\alpha \sigma} \eta_{\beta \lambda}}{4},
\end{gathered}
$$

onde o tensor $I$ é o mesmo definido na Eq. (3.40).

Podemos proceder como fizemos para obter o propagador do glúon no espaço de momento:

$$
\begin{aligned}
M_{\mu \alpha \beta \nu \lambda \sigma}^{a} b(x-y) & =\delta^{a b}\left(\begin{array}{cc}
A_{\mu \nu} & B_{\mu \lambda \sigma} \\
C_{\alpha \beta \nu} & D_{\alpha \beta \lambda \sigma}
\end{array}\right) \int \frac{d^{d} p}{(2 \pi)^{d}} e^{-i p(y-x)} \\
& =\delta^{a b} \int \frac{d^{d} p}{(2 \pi)^{d}}\left(\begin{array}{cc}
A_{\mu \nu} & B_{\mu \lambda \sigma} \\
C_{\alpha \beta \nu} & D_{\alpha \beta \lambda \sigma}
\end{array}\right) e^{-i p(y-x)} \\
& =\delta^{a b} \int \frac{d^{d} p}{(2 \pi)^{d}} e^{-i p(y-x)}\left(\begin{array}{cc}
\tilde{A}_{\mu \nu} & \tilde{B}_{\mu \lambda \sigma} \\
\tilde{C}_{\alpha \beta \nu} & \tilde{D}_{\alpha \beta \lambda \sigma}
\end{array}\right)=\int \frac{d^{d} p}{(2 \pi)^{d}} \tilde{M}_{\mu \alpha \beta \nu \lambda \sigma}^{a}(p) .
\end{aligned}
$$

Podemos então identificar

$$
\tilde{M}_{\mu \alpha \beta \nu \lambda \sigma}^{a b}(p)=\delta^{a b}\left(\begin{array}{cc}
\tilde{A}_{\mu \nu} & \tilde{B}_{\mu \lambda \sigma} \\
\tilde{C}_{\alpha \beta \nu} & \tilde{D}_{\alpha \beta \lambda \sigma}
\end{array}\right)
$$

onde os elementos no espaço de momento são obtidos fazendo $\partial=\partial_{x} \rightarrow+i p$ nas Eqs. (C.32):

$$
\begin{gathered}
\tilde{A}_{\mu \nu}=\frac{1}{\xi} p_{\mu} p_{\nu}, \\
\tilde{B}_{\mu \lambda \sigma}=-i \frac{p_{\lambda} \eta_{\sigma \mu}-p_{\sigma} \eta_{\lambda \mu}}{2}, \\
\tilde{C}_{\alpha \beta \nu}=\frac{p_{\alpha} \eta_{\beta \nu}-p_{\beta} \eta_{\alpha \nu}}{2}, \\
\tilde{D}_{\alpha \beta \lambda \sigma} \equiv \frac{1}{2} I_{\alpha \beta \lambda \sigma} .
\end{gathered}
$$

Pela Eq. (C.2), os propagadores são obtidos pelos elementos da matriz propagador obtida pela inversa da Eq. (C.34) multiplicados pelo fator $-i^{-1}$ (lembrando que obtemos as regras de Feynman usando $i S$.).

$$
\Delta_{\mu \alpha \beta \nu \lambda \sigma}^{a}(p)=i\left(\tilde{M}_{\mu \alpha \beta \nu \lambda \sigma}^{a}\right)^{b}(p)=\left(\begin{array}{cc}
\left\langle A_{\mu}^{a}(-p) A_{\nu}^{b}(p)\right\rangle & \left\langle A_{\mu}^{a}(-p) F_{\lambda \sigma}^{b}(p)\right\rangle \\
\left\langle F_{\alpha \beta}^{a}(-p) A_{\nu}^{b}(p)\right\rangle & \left\langle F_{\alpha \beta}^{a}(-p) F_{\lambda \sigma}^{b}(p)\right\rangle
\end{array}\right) .
$$

A inversa de uma matriz bloco como na Eq. (C.34) pode ser dada por [5]

$$
\left(\begin{array}{ll}
A & B \\
C & D
\end{array}\right)^{-1}=\left(\begin{array}{cc}
X^{-1} & -X^{-1} B D^{-1} \\
-D^{-1} C X^{-1} & D^{-1}+D^{-1} C X^{-1} B D^{-1}
\end{array}\right)
$$


onde $X=A-B D^{-1} C$. Aplicando a identidade acima para a matriz da Eq. (C.34) obtemos

$$
\left(\tilde{M}_{\mu \alpha \beta \nu \lambda \sigma}^{a}\right)^{-1}(p)=-i \delta^{a b}\left(\begin{array}{cc}
P_{\mu \nu}(p) & Q_{\mu \lambda \sigma}(p) \\
Q_{\nu \alpha \beta}(-p) & 2 i\left[I_{\alpha \beta \lambda \sigma}+L_{\alpha \beta \lambda \sigma}(p)\right]
\end{array}\right)
$$

onde $P_{\mu \nu}(p)$ é o propagador do campo de calibre $A_{\mu}^{a}$ definido na Eq. (C.16),

$$
Q_{\mu \lambda \sigma}(p) \equiv-\frac{p_{\lambda} \eta_{\sigma \mu}-p_{\sigma} \eta_{\lambda \mu}}{p^{2}}
$$

e

$$
L_{\alpha \beta \lambda \sigma}(p)=-\frac{1}{2 p^{2}}\left(p^{\alpha} p^{\lambda} g^{\beta \sigma}-p^{\beta} p^{\lambda} g^{\alpha \sigma}-p^{\alpha} p^{\sigma} g^{\beta \lambda}+p^{\beta} p^{\sigma} g^{\alpha \lambda}\right)
$$

Usando a Eq. (C.38) na Eq. (C.36) obtemos os seguintes propagadores

$$
\begin{aligned}
\left\langle A_{\mu}^{a}(-p) A_{\nu}^{b}(p)\right\rangle & =-\frac{i}{p^{2}}\left(\eta_{\mu \nu}-(1-\xi) \frac{p_{\mu} p_{\nu}}{p^{2}}\right) \delta^{a b} \\
\left\langle A_{\mu}^{a}(-p) F_{\lambda \sigma}^{b}(p)\right\rangle & =-\frac{p_{\lambda} \eta_{\sigma \mu}-p_{\sigma} \eta_{\lambda \mu}}{p^{2}} \delta^{a b} \\
\left\langle F_{\alpha \beta}^{a}(-p) A_{\nu}^{b}(p)\right\rangle & =\frac{p_{\alpha} \eta_{\beta \nu}-p_{\beta} \eta_{\alpha \nu}}{p^{2}} \delta^{a b} \\
\left\langle F_{\alpha \beta}^{a}(-p) F_{\lambda \sigma}^{b}(p)\right\rangle & =2 i\left[I_{\alpha \beta \lambda \sigma}+L_{\alpha \beta \lambda \sigma}(p)\right] \delta^{a b}
\end{aligned}
$$

que concordam ${ }^{7}$ com a Ref. [15]. Note que, o propagador para o campo de calibre é o mesmo que o obtido no formalismo de segunda ordem ${ }^{8}$.

No formalismo de primeira ordem só temos um vértice $(F A A)$ que não depende de momento, sendo fácil de se derivar diretamente da lagrangiana efetiva do formalismo de primeira ordem dada na Eq. (3.16). A regra de Feynman para esse vértice é dada por

$$
\mathcal{V}_{\mu \nu \alpha \beta}^{a b c}=-i g f^{a b c} I_{\mu \nu \alpha \beta}
$$

O setor do campo fantasma é o mesmo encontrado no formalismo de segunda ordem assim as regras de Feynman são as mesmas. Sendo assim, as regras de Feynman para os propagadores do

\footnotetext{
${ }^{7}$ Note que o tensor $L$ em [15] é definido diferentemente, o tensor $L$ definido em [15] é igual a $-p^{2} L$ do tensor $L$ definido na Eq. (C.40).

${ }^{8}$ Isso já era esperado devido pela identidade estrutural (3.15).
} 
formalismo de primeira ordem são dadas diagramaticamente por

$$
\begin{aligned}
& \operatorname{mon}_{p}^{a, \nu} P_{\mu \nu}^{a b}(p) \\
& { }_{p}^{a, \mu} b, \lambda \sigma \quad-\frac{p_{\lambda} \eta_{\sigma \mu}-p_{\sigma} \eta_{\lambda \mu}}{p^{2}} \delta^{a b}, \\
& \underset{p}{a, \alpha \beta} \infty^{b, \nu} \quad \frac{p_{\alpha} \eta_{\beta \nu}-p_{\beta} \eta_{\alpha \nu}}{p^{2}} \delta^{a b}, \\
& \frac{a, \alpha \beta \quad b, \lambda \sigma}{p} 2 i\left[I_{\alpha \beta \lambda \sigma}+L_{\alpha \beta \lambda \sigma}(p)\right] \delta^{a b}, \\
& \underset{p}{a+\cdots} \stackrel{b}{p^{2}} \delta^{a b},
\end{aligned}
$$

onde as linhas sólidas representam o campo auxiliar $F_{\mu \nu}^{a}$. Já os vértices (todos momentos estão entrando) são dados por

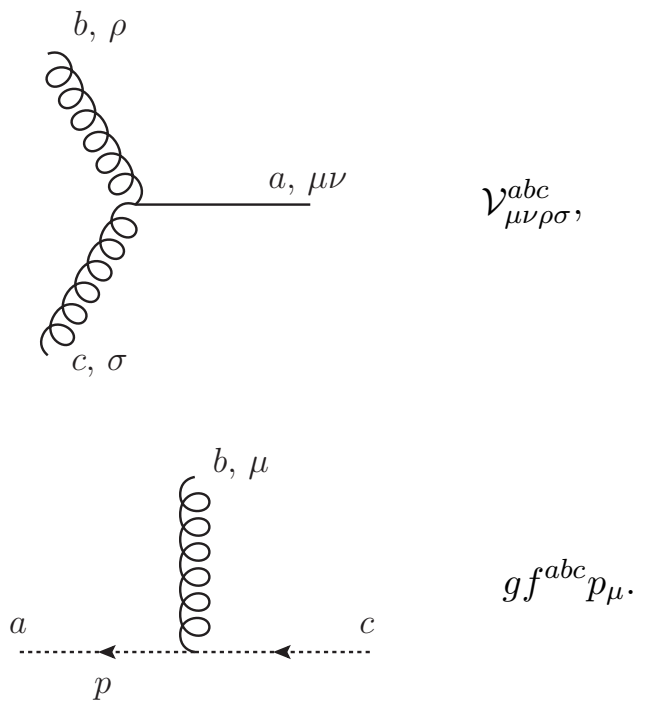

\section{C.3.3 Formalismo diagonal}

A Lagrangiana efetiva do formalismo diagonal é dada por

$$
\begin{aligned}
& \mathcal{L}_{\text {eff }}^{(1 d)}=-\frac{1}{2}\left(\partial_{\mu} A_{\nu}^{a}-\partial_{\nu} A_{\mu}^{a}\right)\left(g f^{a b c} A_{\mu}^{b} A_{\nu}^{c}\right)-\frac{1}{4}\left(\partial_{\mu} A_{\nu}^{a}-\partial_{\nu} A_{\mu}^{a}\right)^{2}+\mathcal{L}_{\mathrm{gf}}(A)+\mathcal{L}_{\mathrm{gh}}(A) \\
& +\frac{1}{4} H^{a \mu \nu} H_{\mu \nu}^{a}-\frac{1}{2} H^{a \mu \nu} h_{\mu \nu}^{a} .
\end{aligned}
$$

Note que o setor do campo de calibre é igual ao encontrado na Eq. (2.10) somente com a exceção da parte quártica no campo de calibre $A_{\mu}^{a}$ que não aparece. Sendo assim, temos as mesmas regras de Feynman para o propagador do campo de calibre $A_{\mu}^{a}$ e o vértice cúbico $(A A A)$, mas sem a presença do vértice $(A A A A)$ no formalismo diagonal. 
Já o setor do campo auxiliar $H_{\mu \nu}^{a}$ possui a mesmo termo de interação encontrado no formalismo diagonal para o campo $F_{\mu \nu}^{a}$, tal que, o vértice $(H A A)$ coincide com o vértice $(F A A)$. Além disso, como já foi mencionado o setor do campo fantasma é o mesmo em todos formalismos e portanto as regras de Feynman para os campos fantasmas coincidem em todas as formulações.

Somente nos resta encontrar o propagador do campo auxiliar $H_{\mu \nu}^{a}$ que pode ser obtido facilmente da Eq. (C.2): momentos estão entrando):

$$
\begin{aligned}
& \underset{p}{a, \mu} \operatorname{mon}^{b, \nu} P_{\mu \nu}^{a b}(p)
\end{aligned}
$$

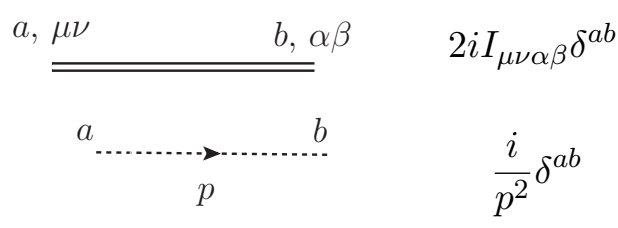

$a, \mu$
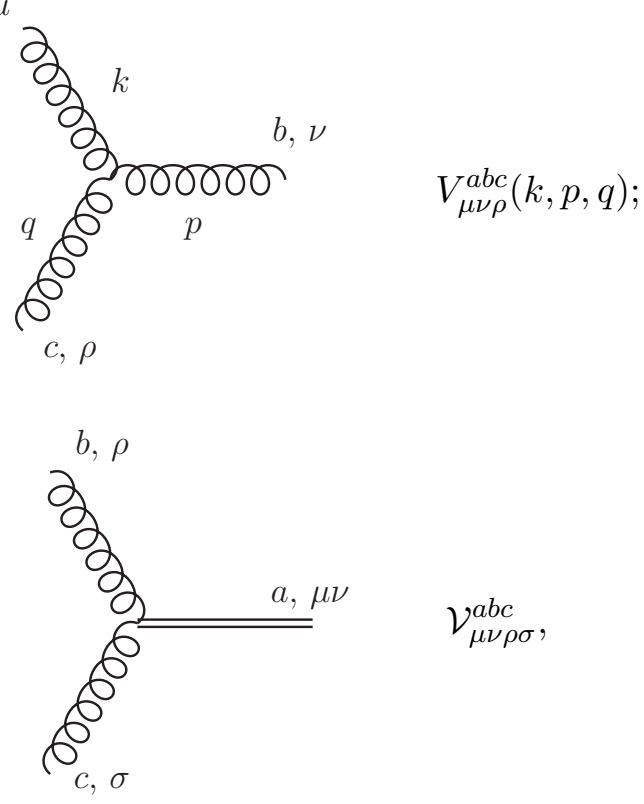

\section{(2)}




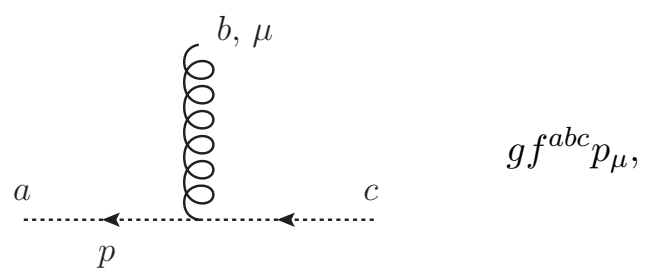

onde as linhas duplas representam o campo auxiliar $H_{\mu \nu}^{a}$.

\section{C.4 Gravitação}

Nessa seção mostramos algumas das regras de Feynman para gravitação. Como para a teoria de YM obteremos as regras a partir de $i S$ invés de $S$ como feito nos artigos [15, 16]. Como referência daremos inicialmente as regras para o setor dos campos fantasmas que são igual em todos os formalismos.

\section{C.4.1 Campos fantasmas $d_{\nu}$ e $\bar{d}_{\mu}$}

Pelos termos do setor fantasma das lagrangianas da gravitação (já com o fator de $i$ )

$$
i \bar{d}_{\mu}\left\{\partial^{2} \eta^{\mu \nu}+\kappa\left[\left(\phi_{, \rho}^{\rho \sigma}\right) \partial_{\sigma} \eta^{\mu \nu}-\left(\phi_{, \rho}^{\rho \mu}\right) \partial^{\nu}+\phi^{\rho \sigma} \partial_{\rho} \partial_{\sigma} \eta^{\mu \nu}-\left(\partial_{\rho} \partial^{\nu} \phi^{\rho \mu}\right)\right]\right\} d_{\nu}
$$

vemos que o propagador do campo fantasma é simplesmente

$$
\mu \quad-\frac{i}{p^{2}} \eta^{\mu \nu}
$$

e o vértice é igual a [16]

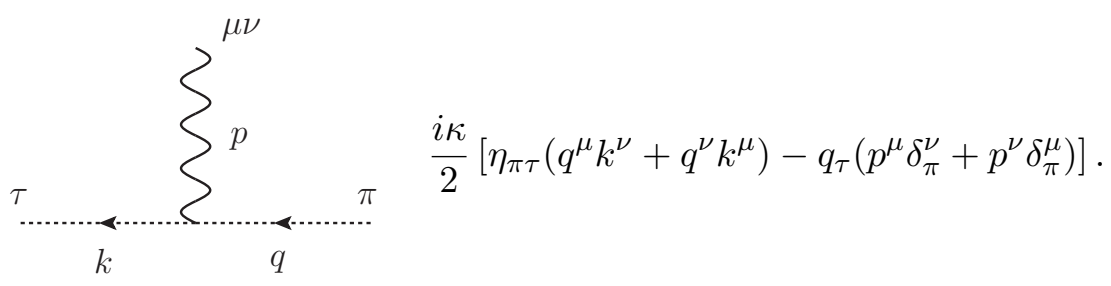

\section{C.4.2 Formalismo diagonal}

Para obter as regras de Feynman decompomos a lagrangiana do formalismo diagonal Eq. (4.35) nos termos bilineares

$$
\begin{gathered}
\frac{1}{2 \kappa^{2}} H_{\mu \nu}^{\lambda} M_{\lambda}^{\mu \nu \pi \tau}(\eta) H_{\pi \tau}^{\rho} \\
-\frac{1}{2} \phi_{, \lambda}^{\mu \nu}\left(M^{-1}\right)_{\mu \nu \pi \tau}^{\lambda \rho}(\eta) \phi_{, \rho}^{\pi \tau}-\frac{1}{2 \alpha}\left(\phi_{, \mu}^{\mu \nu}\right)^{2}
\end{gathered}
$$

e nos termos de interação

$$
\begin{gathered}
\frac{1}{2 \kappa} H_{\mu \nu}^{\lambda} M_{\lambda \sigma}^{\mu \nu \pi \tau}(\phi) H_{\pi \tau}^{\sigma} \\
H_{\mu \nu}^{\lambda} M_{\lambda \sigma}^{\mu \nu \pi \tau}(\phi)\left(M^{-1}\right)_{\pi \tau \gamma \delta}^{\sigma v}(\eta) \phi_{, v}^{\gamma \delta}
\end{gathered}
$$




$$
\frac{\kappa}{2} \phi_{, \rho}^{\alpha \beta}\left(M^{-1}\right)_{\alpha \beta \mu \nu}^{\rho} \lambda(\eta) M_{\lambda}^{\mu \nu \pi \tau} \sigma(\phi)\left(M^{-1}\right)_{\pi \tau \gamma \delta}^{\sigma v}(\eta) \phi_{, v}^{\gamma \delta} .
$$

Os propagadores são obtidos como a inversa dos termos bilineares (com um fator final de $i$, conforme a Eq. (C.2)), tal que, da Eq. (C.53b) obtemos o propagador do campo de calibre $\phi^{\mu \nu}$

$$
\begin{aligned}
\left\langle\phi^{\mu \nu}(-k) \phi^{\pi \tau}(k)\right\rangle & \equiv \mathcal{P}^{\mu \nu \pi \tau}(k)=-i \frac{\eta^{\pi \nu} \eta^{\mu \tau}+\eta^{\pi \mu} \eta^{\nu \tau}-(2-\alpha) \eta^{\pi \tau} \eta^{\mu \nu}}{k^{2}} \\
& +i(1-\alpha) \frac{k^{\pi} k^{\mu} \eta^{\nu \tau}+k^{\pi} k^{\nu} \eta^{\mu \tau}+\eta^{\pi \nu} k^{\mu} k^{\tau}+\eta^{\pi \mu} k^{\nu} k^{\tau}-2 \eta^{\pi \tau} k^{\mu} k^{\nu}-2 k^{\pi} k^{\tau} \eta^{\mu \nu}}{k^{2}}
\end{aligned}
$$

Já o propagador do campo auxiliar obtido a partir da Eq. (C.53a) não depende de momento sendo simplesmente $i \kappa^{2} m^{-1}(m \equiv M(\eta))$. Usando a definição de $m^{-1}$, temos que

$$
\begin{aligned}
\left\langle H_{\mu \nu}^{\lambda}(-k) H_{\pi \tau}^{\rho}(k)\right\rangle \equiv \mathcal{D}_{\mu \nu \pi \tau}^{\lambda \rho} & =\frac{i \kappa^{2}}{4} \eta^{\lambda \rho}\left(\eta_{\pi \mu} \eta_{\tau \nu}+\eta_{\pi \nu} \eta_{\tau \mu}-\frac{2}{(d-2)} \eta_{\mu \nu} \eta_{\pi \tau}\right) \\
& -\frac{i \kappa^{2}}{4}\left(\eta_{\tau \mu} \delta_{\nu}^{\rho} \delta_{\pi}^{\lambda}+\eta_{\pi \mu} \delta_{\nu}^{\rho} \delta_{\tau}^{\lambda}+\eta_{\tau \nu} \delta_{\mu}^{\rho} \delta_{\pi}^{\lambda}+\eta_{\pi \nu} \delta_{\mu}^{\rho} \delta_{\tau}^{\lambda}\right) .
\end{aligned}
$$

Os vértices são obtidos de forma semelhante usando a Eq. (C.12). A base do vértice $(H H \phi)$ pode ser obtida imediata da Eq. (C.54a) sendo igual a $i \mathcal{M} / \kappa$, onde $\mathcal{M}$ é definido como a derivada primeira de $M(\phi)$ em relação a $\phi$. Considerando as permutações de $\mathcal{M}$ podemos obter a regra para o vértice $\left(H_{\mu \nu}^{\lambda} H_{\pi \tau}^{\rho} \phi^{\alpha \beta}\right)$ :

$$
\frac{i}{8 \kappa}\left\{\left[\left(\frac{\delta_{\pi}^{\rho} \delta_{\nu}^{\alpha} \delta_{\tau}^{\beta} \delta_{\mu}^{\lambda}}{d-1}-\delta_{\pi}^{\lambda} \delta_{\nu}^{\alpha} \delta_{\tau}^{\beta} \delta_{\mu}^{\rho}+\alpha \leftrightarrow \beta\right)+\mu \leftrightarrow \nu\right]+\pi \leftrightarrow \tau\right\}+(\mu, \nu, \lambda) \leftrightarrow(\pi, \tau, \rho),
$$

onde $\leftrightarrow$ significa uma permutação de índices. Note que o último termo da Eq. (C.57) é devido a simetria das pernas externa do campo auxiliar $G$. Além disso, vale notar que esse vértice é igual a $(G G \phi)$ já que o termo de interação é o mesmo.

Resta os vértices dependentes do momento: o vértice $(H \phi \phi)$ obtido a partir do termo de interação (C.54b) tem uma base igual a $2 \mathcal{M} \cdot\left(m^{-1} \cdot \partial\right)$. A forma explícita do vértice $\left(H_{\mu \nu}^{\lambda}(p) \phi^{\pi \tau}(q) \phi^{\alpha \beta}(k)\right) \equiv V_{\mu \nu}^{\lambda \pi \tau \alpha \beta}(p, q, k)$ pode ser obtida a partir do vértice (C.57), tal que obtemos

$$
\frac{k^{\theta}}{4}\left\{\left[\left(\frac{\delta_{\mu}^{\pi} \delta_{\nu}^{\lambda}}{d-1} \mathcal{D}_{\theta}^{\alpha \beta \tau \rho}-\delta_{\mu}^{\pi} \mathcal{D}_{\theta}^{\alpha \beta \tau \lambda}+\pi \leftrightarrow \tau\right)+\alpha \leftrightarrow \beta\right]+\mu \leftrightarrow \nu\right\}+(k, \alpha, \beta) \leftrightarrow(q, \pi, \tau) .
$$

No vértice acima não temos na verdade uma dependência em $p$, o momento da perna externa do campo auxiliar $H_{\mu \nu}^{\lambda}$.

Por sua vez, o vértice cúbico $\left(\phi^{\mu \nu}(p) \phi^{\alpha \beta}(q) \phi^{\pi \tau}(k)\right) \equiv V^{\mu \nu \pi \tau \alpha \beta}(p, q, k)$ obtido a partir do termo (C.54c) leva na base $\kappa\left(\partial \cdot m^{-1}\right) \cdot \mathcal{M} \cdot\left(m^{-1} \cdot \partial\right)$, tal que, pode ser obtido a partir da Eq. (C.58). Esse vértice pode ser escrito como

$$
-i \kappa \frac{q^{\kappa} k^{\theta}}{8}\left\{\left[\left(\frac{\mathcal{D}_{\theta}^{\alpha \beta \mu \rho} \mathcal{D}_{\kappa}^{\pi \tau \nu \gamma}}{d-1}-\mathcal{D}_{\kappa}^{\alpha \beta \mu \rho} \mathcal{D}_{\theta}^{\pi \tau \nu \gamma}+\pi \leftrightarrow \tau\right)+\alpha \leftrightarrow \beta\right]+\mu \leftrightarrow \nu\right\}+\cdots,
$$

onde $\cdots$ representa as seis permutações possíveis das pernas externas do campo de calibre $\phi$.

Na forma diagramática, as regras de Feynman para o formalismo diagonal são listadas abaixo: 

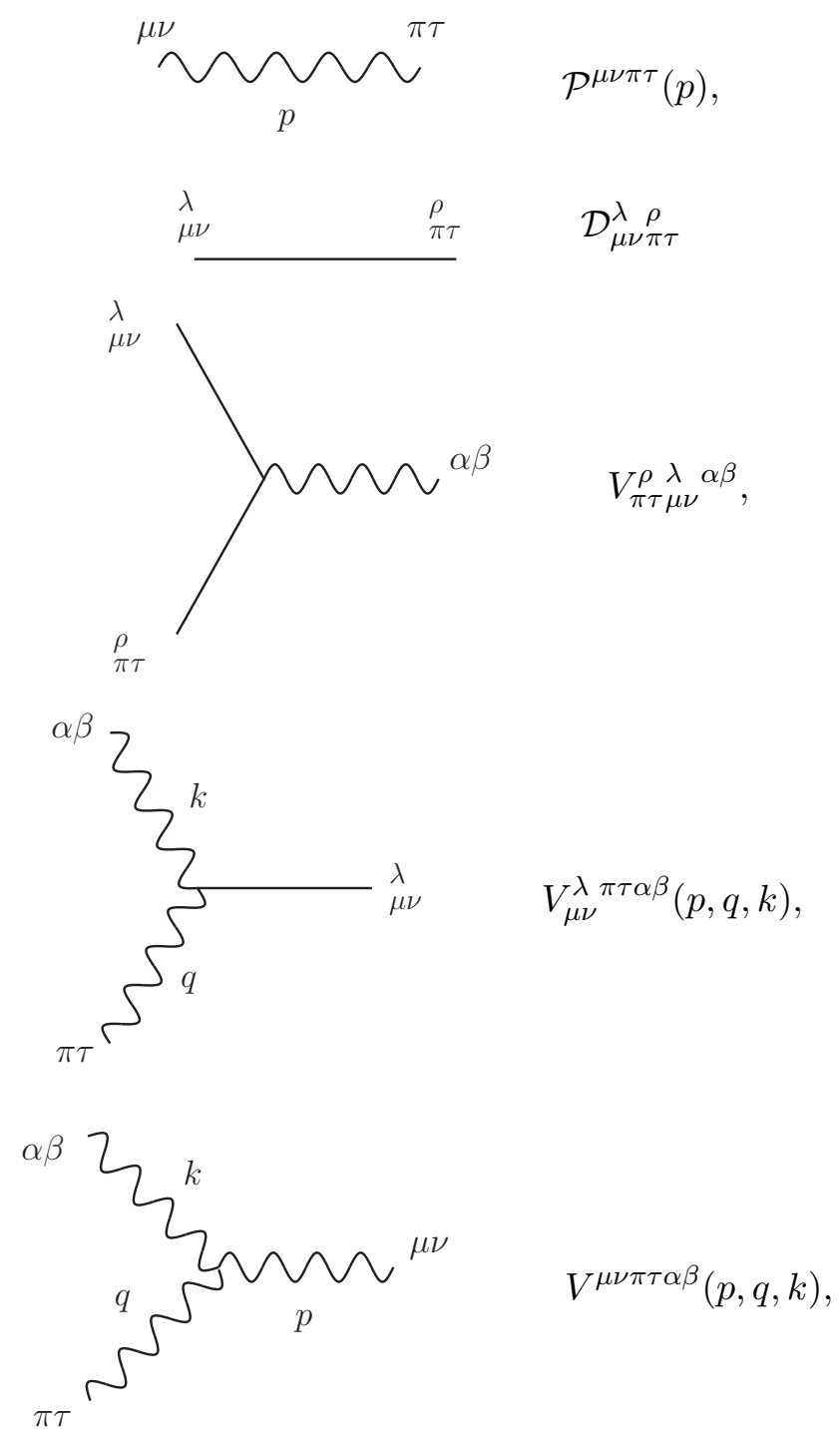

onde linhas onduladas representam o gráviton (campo $\phi^{\mu \nu}$ ), as retas representam o campo auxiliar $H_{\mu \nu}^{\lambda}$ e as linhas tracejadas, os campos fantasmas.

\section{C.4.3 Demais formalismos}

As regras relevantes para o formalismo de segunda ordem são as mesmas encontradas no formalismo diagonal; isso é, tanto o propagador do campo $\phi^{\mu \nu}$, o vértice cúbico $(\phi \phi \phi)$ são iguais [16] aos encontrados no formalismo diagonal.

Já os propagadores do formalismo de primeira ordem, derivados de forma similar aos propagadores da teoria de YM na formulação análoga, são dados por:
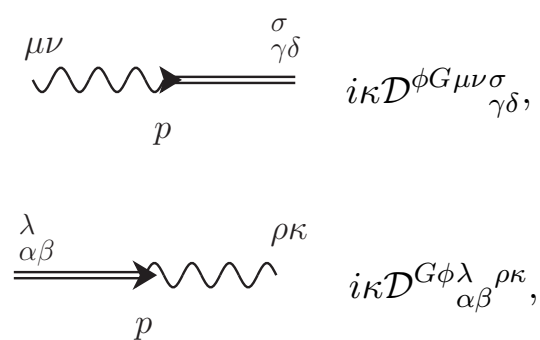


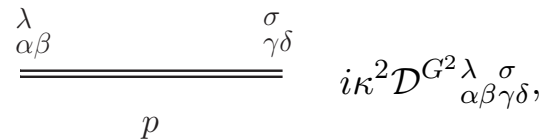

onde linhas duplas, nesse caso, representam o campo auxiliar $G_{\mu \nu}^{\lambda} ; \mathcal{D}_{\alpha \beta}^{G \phi \lambda}{ }^{\rho \kappa}, \mathcal{D}^{\phi G \mu \nu \sigma}{ }_{\gamma \delta}$ e $\mathcal{D}^{G^{2} \lambda}{ }_{\alpha \beta \gamma \delta}^{\sigma}$, são definidos no artigo [5, Eqs. (28-30)]. Ressaltamos que a regra para o vértice $(G G \phi)$ é a mesma do vértice $(H H \phi)$ dada na Eq. (C.57). 



\section{APÊNDICE D}

\section{Método para Computação de Diagramas de 1-LOOP}

Neste apêndice iremos detalhar o procedimento usado para o cálculo dos diagramas a nível de um loop. Nós usamos um software de computação simbólica para sistematizar o procedimento usado em [6] e, em mais detalhes, nos apêndices dos artigos [15, 16]. Esse método consiste em usar regularização dimensional para regularizar as integrais encontradas e redução de PassarinoVeltman para a computação das integrais regularizadas em termos de integrais escalares. No final do apêndice damos um exemplo de código usado.

\section{D.1 Regularização dimensional}

A técnica de regularização dimensional de certa forma é uma técnica de continuação analítica considerando a dimensão como parâmetro livre. Ela é uma ótima ferramenta para a regularização de teorias de calibre [38] já que mantêm as simetrias locais como a invariância de calibre. Iremos nos basear no método que foi desenvolvido independentemente por Giambiagi e Bollini [39]; 't Hooft e Veltman [53].

Para ilustrar considere a integral abaixo [38]:

$$
I[d]=\int d^{d} p_{E} \frac{1}{p_{E}^{2}\left(p_{E}^{2}+m^{2}\right)},
$$

O integrando de $I[d]$ para valores grandes de momento (região ultravioleta) se comporta como $p_{E}^{d-5}$. Assim para $d=4$ (dimensão do espaço tempo $3+1$ ) o integrando vai com $p_{E}^{d-5}=p_{E}^{-1}$ mostrando que $I[4]$ é divergente. Porém, considerando uma dimensão menor: o integrando se comporta como $p_{E}^{-2}$ indicando a convergência de $I[3]$. Logo, podemos considerar $d=4-2 \epsilon$, onde $\epsilon$ é um número complexo e por continuação analítica definir $I[4] \equiv I[4-2 \epsilon]$.

Note que, usamos $p_{E}$ invés de $p$ na Eq. (D.1) pois estamos trabalhando no espaço Euclidiano e não de Minkowski. É fácil compreender a razão, a $I[d]$ no espaço Euclidiano pode ser trivialmente 
computada já que depende somente de $p_{E}^{2}$, enquanto a integral no espaço de Minkowski é mais complexa de se computar como discutimos a seguir.

A integral (D.1) no espaço de Minkowski é obtida fazendo uma rotação de Wick:

$$
p_{E}^{0}=-i p^{0}
$$

tal que,

$$
I[d]=-i \int d^{d} p \frac{1}{p^{2}\left(p^{2}-m^{2}+i \varepsilon\right)},
$$

onde temos que adicionar uma fase $i \varepsilon$ para obter o resultado com a correta ordenação temporal, o resultado final é obtido fazendo $\varepsilon \rightarrow 0$. Fica claro que a integral no espaço de Minkowski é mais complexa de computar, ainda mais porque não precisamos nos preocupar com os polos do integrando da Eq. (D.3) e a escolha correta de contorno (que na verdade é remediada pela adição de $i \varepsilon)$ no espaço Euclidiano.

\section{D.1.1 Uma integral básica}

Como um exemplo do uso da regularização dimensional computaremos uma integral que aparece em diversos cálculos de diagramas de um loop:

$$
I_{n}[d] \equiv \int \frac{d^{d} p}{(2 \pi)^{d}} \frac{1}{\left(p^{2}-\Delta\right)^{n}},
$$

onde ainda estamos no espaço de Minkowski.

Para computar $I_{n}[d]$ fazemos uma rotação de Wick, tal que, $p^{2}=-p_{E}^{2}$ e $d^{d} p=i d^{d} p_{E}$, tal que, expressão acima pode ser reescrita no espaço Euclidiano como

$$
I_{n}[d]=\frac{i}{(-1)^{n}} \int \frac{d^{d} p_{E}}{(2 \pi)^{d}} \frac{1}{\left(p_{E}^{2}+\Delta\right)^{n}},
$$

onde já podemos considerar a integração partindo para coordenadas esféricas generalizadas em $d$ dimensões com $d^{d} p_{E}=p_{E}^{d-1} d \Omega(\Omega$ é a generalização do ângulo sólido):

$$
I_{n}[d]=i^{1-2 n} \int \frac{d^{d} p_{E}}{(2 \pi)^{d}} \frac{1}{\left(p_{E}^{2}+\Delta\right)^{n}}=i^{1-2 n} \int d \Omega \int \frac{d p_{E}}{(2 \pi)^{d}} \frac{p_{E}^{d-1}}{\left(p_{E}^{2}+\Delta\right)^{n}}
$$

A integração do ângulo sólido é trivial e obtemos

$$
I_{n}[d]=i^{1-2 n} \frac{2 \pi^{d / 2}}{\Gamma(d / 2)} \int \frac{d p_{E}}{(2 \pi)^{d}} \frac{p_{E}^{d-1}}{\left(p_{E}^{2}+\Delta\right)^{n}}
$$

onde $\Gamma(x)$ é a função Gamma.

Partindo para a integral no momento, podemos fazer a substituição $t=\Delta /\left(p_{E}^{2}+\Delta\right)$ com $d t=-\Delta d t /\left(2 p t^{2}\right)$, tal que:

$$
I_{n}[d]=i^{1-2 n} \frac{\pi^{d / 2}}{\Gamma(d / 2)} \frac{1}{(2 \pi)^{d}} \Delta^{d / 2-n} \int_{0}^{1} d t t^{n-d / 2-1}(1-t)^{d / 2-1},
$$

onde identificamos a integral na variável $t$ como a função Beta $\mathrm{B}(n-d / 2, d / 2)$. Escrevendo a função Beta em termo da função Gamma

$$
\mathrm{B}\left(n-\frac{d}{2}, \frac{d}{2}\right)=\frac{\Gamma(n-d / 2) \Gamma(d / 2)}{\Gamma(n)}
$$


e substituindo na Eq. (D.8) obtemos

$$
I_{n}[d]=i^{1-2 n} \frac{2 \pi^{d / 2}}{\Gamma(d / 2)} \frac{1}{(2 \pi)^{d}} \Delta^{d / 2-n} \frac{\Gamma(n-d / 2)}{\Gamma(n)}=\frac{i^{1-2 n}}{(4 \pi)^{d / 2}} \Delta^{d / 2-n} \frac{\Gamma(n-d / 2)}{\Gamma(n)} .
$$

Ressaltamos que o resultado obtido para $I_{n}[d]$ por regularização dimensional é claramente bem definido para $d=4$ como esperado.

\section{D.1.2 Integral de potências}

A integral que usaremos para a computação de diagramas é dado por potências negativas de momento:

$$
I_{l m} \equiv \int \frac{d^{d} p}{(2 \pi)^{d}} \frac{1}{\left(p^{2}\right)^{l}\left(q^{2}\right)^{m}}
$$

$\operatorname{com} q=p+k$.

Para computar a integral acima precisamos introduzir os parâmetros de Feynman:

$$
\frac{1}{A^{l} B^{m}}=\frac{\Gamma(l+m)}{\Gamma(l) \Gamma(m)} \int_{0}^{1} d x \frac{x^{l-1}(1-x)^{m-1}}{[x A+(1-x) B]^{l+m}} .
$$

Com $A=q^{2}$ e $B=p^{2}$ na Eq. (D.12) e substituindo na Eq. (D.11), temos que

$$
I_{l m}=\frac{\Gamma(l+m)}{\Gamma(l) \Gamma(m)} \int \frac{d^{d} p}{(2 \pi)^{d}} \int_{0}^{1} d x \frac{x^{l-1}(1-x)^{m-1}}{\left[x q^{2}+(1-x) p^{2}\right]^{l+m}},
$$

usando $q=p+k$ podemos fazer o shift $p_{\mu}=P_{\mu}-x k_{\mu}$ obtendo

$$
I_{l m}=\frac{\Gamma(l+m)}{\Gamma(l) \Gamma(m)} \int \frac{d^{d} P}{(2 \pi)^{d}} \int_{0}^{1} d x \frac{x^{l-1}(1-x)^{m-1}}{\left(P^{2}-\Delta\right)^{l+m}},
$$

onde $\Delta=-x(1-x) k^{2}$ e a integral em $P$ está na forma da integral básica (D.4).

Pela Eq. (D.10) com $n=l+m$, temos que

$$
I_{l m}=\frac{\Gamma(d / 2)}{\Gamma(l) \Gamma(m)} \frac{i(-1)^{l+m}}{(4 \pi)^{d / 2}}\left(k^{2}\right)^{d / 2-l-m} \int_{0}^{1} d x \frac{x^{l-1}(1-x)^{m-1}}{[-x(1-x)]^{l+m}} .
$$

A integral em $x$ na equação acima pode ser simplificada, tal que,

$$
I_{l m}=i(-1)^{2(l+m)+d / 2} \frac{\Gamma(l+m-d / 2)}{\Gamma(l) \Gamma(m)} \frac{\left(k^{2}\right)^{d / 2-l-m}}{(4 \pi)^{d / 2}} \int_{0}^{1} d x x^{d / 2-m-1}(1-x)^{d / 2-l-1},
$$

chegando na forma da função beta $\mathrm{B}(d / 2-m, d / 2-l)$. Finalmente, usando a Eq. (D.9), chegamos na expressão ${ }^{1}$ em termo de $l, m$ e $d$, e o momento $k^{2}$ :

$$
I_{l m}=i^{1+d} \frac{\left(k^{2}\right)^{d / 2-l-m}}{(4 \pi)^{d / 2}} \frac{\Gamma(l+m-d / 2)}{\Gamma(l) \Gamma(m)} \frac{\Gamma(d / 2-m) \Gamma(d / 2-l)}{\Gamma(d-m-l)}
$$

Nós vamos precisar de somente alguns valores de $l$ e $m$. Abaixo calculamos $I_{l m}$ para $l$ e $m$ tomando valores de 1 e 2 usando o resultado obtido na Eq. (D.17):

\footnotetext{
${ }^{1}$ Conforme a expressão [15, eq. B5].
} 
- Para $l=m=1$, temos que

$$
\begin{aligned}
I_{11} & =\frac{i^{1+d}}{(4 \pi)^{d / 2}}\left(k^{2}\right)^{d / 2-2} \frac{\Gamma(2-d / 2)}{\Gamma(1) \Gamma(1)} \frac{\Gamma(d / 2-1) \Gamma(d / 2-1)}{\Gamma(d-2)} \\
& =i^{1+d} \frac{\left(k^{2}\right)^{d / 2-2}}{(4 \pi)^{d / 2}} \frac{\Gamma(2-d / 2) \Gamma(d / 2-1)^{2}}{\Gamma(d-2)} .
\end{aligned}
$$

- Com $l=1$ e $m=2$ ou $l=2$ e $m=1$ as integrais coincidem:

$$
I_{12}=I_{21}=\frac{3-d}{k^{2}} I_{11}
$$

- E para $l=m=2$ :

$$
I_{22}=\frac{(3-d)(6-d)}{k^{4}} I_{11}
$$

Além do resultado para $d$ geral é interessante saber o comportamento da divergência ultravioleta (UV). Para isso expandimos em torno de $d=4$, considerando $d=4-2 \epsilon$ na expressão para $I_{11}$ na Eq. (D.18) obtemos

$$
I_{11}=\frac{i}{16 \pi^{2} \epsilon}+\cdots,
$$

onde só explicitamos a parte do polo UV ( $\cdots$ são termos finitos):

$$
I_{\mathrm{UV}}=\frac{i}{16 \pi^{2} \epsilon} .
$$

\section{D.2 Redução de Passarino-Veltman}

Os diagramas de um loop em teoria de calibre não massivas levam à integrais tensoriais. Podemos reduzir essas integrais à integrais escalares usando o método de redução de Passarino-Veltman [54]. Essas integrais são reduzidas tal que não tenhamos nenhuma potência do momento interno no numerador. Tais integrais escalares são mais simples de calcular. Embora, em contrapartida, precisamos escolher uma base tensorial adequada para a decomposição da integral tensorial.

Nessa seção detalharemos o procedimento usado em [15, 16] para a computações dessas integrais de um loop. Conforme é mencionado em [16] esse procedimento é um exemplo de Redução de Passarino-Veltman.

\section{D.2.1 Procedimento geral}

Por simplicidade, consideremos a forma da autoenergia de um campo não massivo (como é o caso dos campos de calibre considerados nesse trabalho) [16]

$$
\Pi^{a b}(k)=\int \frac{d^{d} p}{(2 \pi)^{d}} I^{a b}(p, q)
$$

onde $a$ e $b$ são superíndices que representam uma coleção de índices de Lorentz ou de cor, e como anteriormente $q=p+k$. 
Pelas simetrias de $\Pi^{a b}(k)$ podemos encontrar uma base tensorial tal que possamos decompor a autoenergia como

$$
\Pi^{a b}(k)=\sum_{i=1}^{n} c_{i} T_{i}^{a b}(k)
$$

onde temos $n$ tensores $\left\{T_{i}^{a b}(k)\right\}$. Para obter as componentes da autoenergia nessa base tensorial contraímos a Eq. (D.24) pelos $n$ tensores $T_{j}^{a b}(k)$ obtendo um sistema linear de $n$ equações:

$$
\Pi^{a b}(k) T_{j}^{a b}(k)=\sum_{i=1}^{n} c_{i} T_{i}^{a b}(k) T_{j}^{a b}(k),
$$

onde os coeficientes $c_{i}$ são a solução do sistema linear de equações acima.

Para computar as integrais escalares

$$
\Pi^{a b}(k) T_{j}^{a b}(k)=\int \frac{d^{d} p}{(2 \pi)^{d}} I^{a b}(p, q) T_{j}^{a b}(k)
$$

podemos simplificar o integrando usando as relações (conforme [54, eq. D5] fazendo $m \rightarrow 0$ )

$$
\begin{aligned}
& p \cdot k=\frac{1}{2}\left(q^{2}-p^{2}-k^{2}\right), \\
& q \cdot k=\frac{1}{2}\left(q^{2}-p^{2}+k^{2}\right), \\
& p \cdot q=\frac{1}{2}\left(p^{2}+q^{2}-k^{2}\right) .
\end{aligned}
$$

De tal forma que, conforme [16], as integrais escalares na Eq. (D.26) tomam a forma de $I_{l m}$ definido na Eq. (D.11). Isso é consistente já que não há nenhuma potência do momento interno no numerador do integrando de $I_{l m}$ como espera-se após o procedimento de redução de PassarinoVeltman.

Assim os coeficientes $c_{i}$, solução do sistema linear dado na Eq. (D.25), são obtidos em termo de $I_{l m}$. Isso é, finalmente podemos determinar o resultado da integral tensorial, nesse caso, a autoenergia, em termo de integrais escalares.

De acordo com [16] temos uma grande simplificação das integrais escalares $I_{l m}$ (no caso de teorias de calibre com um calibre geral) pelo uso da regularização dimensional, pois os únicos valores de $l$ e $m$ para $I_{l m}$ não se anular são 1,2. Os demais valores de $l$ e $m$ são de diagramas do tipo "tadpole" que no caso devem realmente ser nulos. Por exemplo, se $l$ ou $m$ são zero, $I_{l m}$ se reduz a integral $I_{l+m}[d]$ com $\Delta=0$ que pela Eq. (D.10) deve se anular. ${ }^{2}$

\section{D.3 Bases tensoriais}

Nessa seção vamos deixar como referência as bases tensoriais usadas para computar os diagramas de um loop.

\footnotetext{
${ }^{2}$ Esse tipo de integral não possui escala de momento, sendo assim por análise dimensional podemos ver que devem se anular. Além disso, vale mencionar que também podemos ter a presença de divergências no infravermelho (IR, pequenos momentos). No entanto equiparando as divergências UV e IR, ajustando os parâmetros de regularização $\epsilon_{\mathrm{UV}}$ e $\epsilon_{\mathrm{IR}}$, verifica-se que o resultado é nulo.
} 


\section{D.3.1 Teoria de Yang-Mills}

No caso da teoria de YM usamos $\left(k_{\mu} \eta_{\nu \rho}-k_{\nu} \eta_{\mu \rho}\right) \delta^{a b}$ como o único tensor de base para integrais que possuem a simetria de $\left\langle H_{\mu \nu}^{a} A_{\rho}^{b}\right\rangle$. E os tensores $I_{\mu \nu \alpha \beta}$ e $L_{\mu \nu \alpha \beta}$ que formam uma base com a simetria de $\left\langle H_{\mu \nu}^{a} H_{\alpha \beta}^{b}\right\rangle$.

\section{D.3.2 Gravitação}

Para gravitação usamos duas bases: uma adequada para a simetria de $\left\langle H_{\mu \nu}^{\lambda} \phi^{\pi \tau}\right\rangle$ e outra para $\left\langle H_{\mu \nu}^{\lambda} H_{\pi \tau}^{\rho}\right\rangle$.

- Base de acordo com a simetria de $\left\langle H_{\mu \nu}^{\lambda} \phi^{\pi \tau}\right\rangle$ :

$$
\begin{aligned}
& \left(T_{1}^{H \phi}\right)_{\mu \nu}^{\lambda \pi \tau}=\frac{1}{4}\left(k^{\pi} \delta_{\nu}^{\lambda} \delta_{\mu}^{\tau}+k^{\pi} \delta_{\mu}^{\lambda} \delta_{\nu}^{\tau}+\delta_{\nu}^{\pi} k^{\tau} \delta_{\mu}^{\lambda}+\delta_{\mu}^{\pi} k^{\tau} \delta_{\nu}^{\lambda}\right) \\
& \left(T_{2}^{H \phi}\right)_{\mu \nu}^{\lambda \pi \tau}=\frac{1}{2} k^{\lambda}\left(\delta_{\nu}^{\pi} \delta_{\mu}^{\tau}+\delta_{\mu}^{\pi} \delta_{\nu}^{\tau}\right) \\
& \left(T_{3}^{H \phi}\right)_{\mu \nu}^{\lambda \pi \tau}=k^{\lambda} \eta^{\pi \tau} \eta_{\mu \nu} \\
& \left(T_{4}^{H \phi}\right)_{\mu \nu}^{\lambda \pi \tau}=\frac{1}{2} \eta_{\mu \nu}\left(k^{\pi} \eta^{\lambda \tau}+\eta^{\lambda \pi} k^{\tau}\right) \\
& \left(T_{5}^{H \phi}\right)_{\mu \nu}^{\lambda \pi \tau}=\frac{1}{4}\left(\delta_{\nu}^{\pi} k_{\mu} \eta^{\lambda \tau}+\eta^{\lambda \pi} k_{\mu} \delta_{\nu}^{\tau}+\delta_{\mu}^{\pi} k_{\nu} \eta^{\lambda \tau}+\eta^{\lambda \pi} k_{\nu} \delta_{\mu}^{\tau}\right) \\
& \left(T_{6}^{H \phi}\right)_{\mu \nu}^{\lambda \pi \tau}=\frac{1}{2} \eta^{\pi \tau}\left(k_{\mu} \delta_{\nu}^{\lambda}+k_{\nu} \delta_{\mu}^{\lambda}\right) \\
& \left(T_{7}^{H \phi}\right)_{\mu \nu}^{\lambda \pi \tau}=\frac{1}{2 k^{2}} k^{\pi} k^{\tau}\left(k_{\mu} \delta_{\nu}^{\lambda}+k_{\nu} \delta_{\mu}^{\lambda}\right) \\
& \left(T_{8}^{H \phi}\right)_{\mu \nu}^{\lambda \pi \tau}=\frac{1}{4 k^{2}} k^{\lambda}\left(k^{\pi} k_{\mu} \delta_{\nu}^{\tau}+k^{\pi} k_{\nu} \delta_{\mu}^{\tau}+\delta_{\nu}^{\pi} k_{\mu} k^{\tau}+\delta_{\mu}^{\pi} k_{\nu} k^{\tau}\right) \\
& \left(T_{9}^{H \phi}\right)_{\mu \nu}^{\lambda \pi \tau}=\frac{1}{2 k^{2}} k_{\mu} k_{\nu}\left(k^{\pi} \eta^{\lambda \tau}+\eta^{\lambda \pi} k^{\tau}\right) \\
& \left(T_{10}^{H \phi}\right)_{\mu \nu}^{\lambda \pi \tau}=\frac{1}{k^{4}} k^{\lambda} k_{\mu} k_{\nu} k^{\pi} k^{\tau} \\
& \left(T_{11}^{H \phi}\right)_{\mu \nu}^{\lambda \pi \tau}=\frac{1}{k^{2}} k^{\lambda} \eta_{\mu \nu} k^{\pi} k^{\tau} \\
& \left(T_{12}^{H \phi}\right)_{\mu \nu}^{\lambda \pi \tau}=\frac{1}{k^{2}} k^{\lambda} k_{\mu} k_{\nu} \eta^{\pi \tau}
\end{aligned}
$$

- Base de acordo com a simetria de $\left\langle H_{\mu \nu}^{\lambda} H_{\pi \tau}^{\rho}\right\rangle$ :

$$
\begin{aligned}
&\left(T_{1}^{H H}\right)_{\mu \nu \pi \tau}^{\lambda}=\frac{1}{4}\left(\delta_{\pi}^{\rho} \delta_{\nu}^{\lambda} \eta_{\mu \tau}+\delta_{\pi}^{\rho} \delta_{\mu}^{\lambda} \eta_{\nu \tau}+\eta_{\pi \nu} \delta_{\mu}^{\lambda} \delta_{\tau}^{\rho}+\eta_{\pi \mu} \delta_{\nu}^{\lambda} \delta_{\tau}^{\rho}\right), \\
&\left(T_{2}^{H H}\right)_{\mu \nu \pi \tau}^{\lambda \rho}=\frac{1}{2} \eta^{\lambda \rho}\left(\eta_{\pi \nu} \eta_{\mu \tau}+\eta_{\pi \mu} \eta_{\nu \tau}\right), \\
&\left(T_{3}^{H H}\right)_{\mu \nu \pi \tau}^{\lambda \rho}=\eta_{\pi \tau} \eta^{\lambda \rho} \eta_{\mu \nu}, \\
&\left(T_{4}^{H H}\right)_{\mu \nu \pi \tau}^{\lambda \rho}=\frac{1}{4}\left(\eta_{\pi \nu} \delta_{\tau}^{\lambda} \delta_{\mu}^{\rho}+\delta_{\pi}^{\lambda} \delta_{\mu}^{\rho} \eta_{\nu \tau}+\eta_{\pi \mu} \delta_{\tau}^{\lambda} \delta_{\nu}^{\rho}+\delta_{\pi}^{\lambda} \eta_{\mu \tau} \delta_{\nu}^{\rho}\right), \\
&\left(T_{5}^{H H}\right)_{\mu \nu \pi \tau}^{\lambda \rho}=\frac{1}{4}\left(\delta_{\pi}^{\rho} \delta_{\tau}^{\lambda} \eta_{\mu \nu}+\delta_{\pi}^{\lambda} \eta_{\mu \nu} \delta_{\tau}^{\rho}+\eta_{\pi \tau} \delta_{\nu}^{\lambda} \delta_{\mu}^{\rho}+\eta_{\pi \tau} \delta_{\mu}^{\lambda} \delta_{\nu}^{\rho}\right), \\
&\left(T_{6}^{H H}\right)_{\mu \nu \pi \tau}^{\lambda \rho}=\frac{1}{4 k^{4}}\left(k_{\pi} k^{\lambda} k_{\mu} k_{\nu} \delta_{\tau}^{\rho}+\delta_{\pi}^{\rho} k^{\lambda} k_{\mu} k_{\nu} k_{\tau}+k_{\pi} k_{\mu} k^{\rho} k_{\tau} \delta_{\nu}^{\lambda}+k_{\pi} k_{\nu} k^{\rho} k_{\tau} \delta_{\mu}^{\lambda}\right),
\end{aligned}
$$




$$
\begin{aligned}
& \left(T_{7}^{H H}\right)_{\mu \nu \pi \tau}^{\lambda \rho}=\frac{1}{4 k^{4}} k^{\lambda} k^{\rho}\left(k_{\pi} k_{\mu} \eta_{\nu \tau}+k_{\pi} k_{\nu} \eta_{\mu \tau}+\eta_{\pi \nu} k_{\mu} k_{\tau}+\eta_{\pi \mu} k_{\nu} k_{\tau}\right), \\
& \left(T_{8}^{H H}\right)_{\mu \nu \pi \tau}^{\lambda \rho}=\frac{1}{4 k^{4}}\left(k_{\pi} k_{\mu} k_{\nu} k^{\rho} \delta_{\tau}^{\lambda}+\delta_{\pi}^{\lambda} k_{\mu} k_{\nu} k^{\rho} k_{\tau}+k_{\pi} k^{\lambda} k_{\mu} k_{\tau} \delta_{\nu}^{\rho}+k_{\pi} k^{\lambda} k_{\nu} k_{\tau} \delta_{\mu}^{\rho}\right), \\
& \left(T_{9}^{H H}\right)_{\mu \nu \pi \tau}^{\lambda \rho}=\frac{1}{2 k^{4}} k^{\lambda} k^{\rho}\left(\eta_{\pi \tau} k_{\mu} k_{\nu}+k_{\pi} k_{\tau} \eta_{\mu \nu}\right), \\
& \left(T_{10}^{H H}\right)_{\mu \nu \pi \tau}^{\lambda \rho}=\frac{1}{4 k^{2}}\left(k_{\pi} \delta_{\tau}^{\rho}+\delta_{\pi}^{\rho} k_{\tau}\right)\left(k_{\mu} \delta_{\nu}^{\lambda}+k_{\nu} \delta_{\mu}^{\lambda}\right), \\
& \left(T_{11}^{H H}\right)_{\mu \nu \pi \tau}^{\lambda \rho}=\frac{1}{4 k^{2}} \eta^{\lambda \rho}\left(k_{\pi} k_{\mu} \eta_{\nu \tau}+k_{\pi} k_{\nu} \eta_{\mu \tau}+\eta_{\pi \nu} k_{\mu} k_{\tau}+\eta_{\pi \mu} k_{\nu} k_{\tau}\right), \\
& \left(T_{12}^{H H}\right)_{\mu \nu \pi \tau}^{\lambda \rho}=\frac{1}{4 k^{2}}\left(k_{\pi} \delta_{\tau}^{\lambda}+\delta_{\pi}^{\lambda} k_{\tau}\right)\left(k_{\mu} \delta_{\nu}^{\rho}+k_{\nu} \delta_{\mu}^{\rho}\right), \\
& \left(T_{13}^{H H}\right)_{\mu \nu \pi \tau}^{\lambda}=\frac{1}{4 k^{2}}\left(\delta_{\pi}^{\rho} k_{\mu} k_{\nu} \delta_{\tau}^{\lambda}+\delta_{\pi}^{\lambda} k_{\mu} k_{\nu} \delta_{\tau}^{\rho}+k_{\pi} k_{\tau} \delta_{\nu}^{\lambda} \delta_{\mu}^{\rho}+k_{\pi} k_{\tau} \delta_{\mu}^{\lambda} \delta_{\nu}^{\rho}\right), \\
& \left(T_{14}^{H H}\right)_{\mu \nu \pi \tau}^{\lambda \rho}=\frac{1}{2 k^{2}} \eta^{\lambda \rho}\left(\eta_{\pi \tau} k_{\mu} k_{\nu}+k_{\pi} k_{\tau} \eta_{\mu \nu}\right), \\
& \left(T_{15}^{H H}\right)_{\mu \nu \pi \tau}^{\lambda \rho}=\frac{1}{8 k^{2}}\left(\delta_{\pi}^{\rho} k^{\lambda} k_{\mu} \eta_{\nu \tau}+k_{\pi} k^{\rho} \delta_{\mu}^{\lambda} \eta_{\nu \tau}+\delta_{\pi}^{\rho} k^{\lambda} k_{\nu} \eta_{\mu \tau}+\eta_{\pi \nu} k^{\lambda} k_{\mu} \delta_{\tau}^{\rho}\right. \\
& \left.+\eta_{\pi \mu} k^{\lambda} k_{\nu} \delta_{\tau}^{\rho}+k_{\pi} k^{\rho} \delta_{\nu}^{\lambda} \eta_{\mu \tau}+\eta_{\pi \nu} k^{\rho} k_{\tau} \delta_{\mu}^{\lambda}+\eta_{\pi \mu} k^{\rho} k_{\tau} \delta_{\nu}^{\lambda}\right) \\
& \left(T_{16}^{H H}\right)_{\mu \nu \pi \tau}^{\lambda \rho}=\frac{1}{2 k^{2}} k^{\lambda} k^{\rho}\left(\eta_{\pi \nu} \eta_{\mu \tau}+\eta_{\pi \mu} \eta_{\nu \tau}\right), \\
& \left(T_{17}^{H H}\right)_{\mu \nu \pi \tau}^{\lambda \rho}=\frac{1}{4 k^{2}}\left(\eta_{\pi \tau} k^{\lambda} k_{\mu} \delta_{\nu}^{\rho}+\eta_{\pi \tau} k^{\lambda} k_{\nu} \delta_{\mu}^{\rho}+k_{\pi} k^{\rho} \delta_{\tau}^{\lambda} \eta_{\mu \nu}+\delta_{\pi}^{\lambda} k^{\rho} k_{\tau} \eta_{\mu \nu}\right), \\
& \left(T_{18}^{H H}\right)_{\mu \nu \pi \tau}^{\lambda \rho}=\frac{1}{8 k^{2}}\left(k_{\pi} k^{\lambda} \eta_{\mu \tau} \delta_{\nu}^{\rho}+\eta_{\pi \mu} k^{\lambda} k_{\tau} \delta_{\nu}^{\rho}+k_{\pi} k^{\lambda} \delta_{\mu}^{\rho} \eta_{\nu \tau}+\eta_{\pi \nu} k_{\mu} k^{\rho} \delta_{\tau}^{\lambda}\right. \\
& \left.+\delta_{\pi}^{\lambda} k_{\mu} k^{\rho} \eta_{\nu \tau}+\eta_{\pi \mu} k_{\nu} k^{\rho} \delta_{\tau}^{\lambda}+\delta_{\pi}^{\lambda} k_{\nu} k^{\rho} \eta_{\mu \tau}+\eta_{\pi \nu} k^{\lambda} k_{\tau} \delta_{\mu}^{\rho}\right), \\
& \left(T_{19}^{H H}\right)_{\mu \nu \pi \tau}^{\lambda \rho}=\frac{1}{4 k^{2}}\left(k_{\pi} k^{\lambda} \eta_{\mu \nu} \delta_{\tau}^{\rho}+\eta_{\pi \tau} k_{\mu} k^{\rho} \delta_{\nu}^{\lambda}+\eta_{\pi \tau} k_{\nu} k^{\rho} \delta_{\mu}^{\lambda}+\delta_{\pi}^{\rho} k^{\lambda} k_{\tau} \eta_{\mu \nu}\right), \\
& \left(T_{20}^{H H}\right)_{\mu \nu \pi \tau}^{\lambda \rho}=\frac{1}{k^{6}} k_{\pi} k^{\lambda} k_{\mu} k_{\nu} k^{\rho} k_{\tau}, \\
& \left(T_{21}^{H H}\right)_{\mu \nu \pi \tau}^{\lambda \rho}=\frac{1}{k^{4}} k_{\pi} k_{\mu} k_{\nu} k_{\tau} \eta^{\lambda \rho}, \\
& \left(T_{22}^{H H}\right)_{\mu \nu \pi \tau}^{\lambda \rho}=\frac{1}{k^{2}} \eta_{\pi \tau} k^{\lambda} k^{\rho} \eta_{\mu \nu} .
\end{aligned}
$$

\section{D.4 Exemplo de código}

Para computar os diagramas de um loop e os diagramas pinched usamos o software Mathematica 12 e o pacote FeynCalc [55]. No código abaixo usamos a Redução de Passarino-Veltman para obter integrais em uma dimensão $d$ qualquer. Usamos uma base tensorial adequada ${ }^{3}$ para obter os diagramas da Figs. 6.4 e 6.6(a) verificando que a identidade estrutural (3.64b) é válida em qualquer dimensão e qualquer calibre no nível de um loop.

A estrutura do código é praticamente a mesma para demais identidades, sendo necessário ajustar, obviamente, a definição do integrando do diagrama de um loop, e usar uma base tensorial adequada.

\footnotetext{
${ }^{3}$ Aqui consideramos um base mais geral que a mencionada na seção D.3.1 para ilustrar o procedimento geral. No entanto para uma computação eficiente é necessário considerar as simetrias, tal que, a base seja mais concisa possível.
} 


\section{Verificação da Identidade (3.64b)}

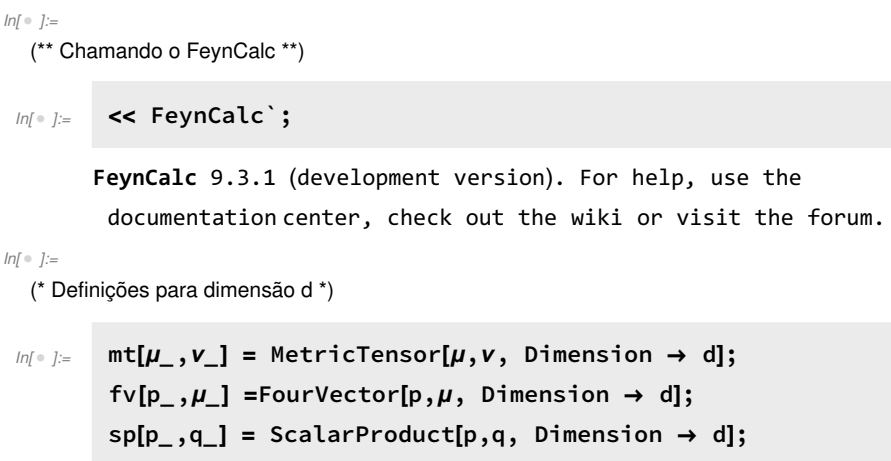

- Regras de Feynman para o formalismo de segunda ordem

- Regras de Feynman para o formalismo diagonal

$\operatorname{In}[\circ]:=\quad \mathrm{IF}\left[\mathrm{m} 1_{-}, \mathrm{m} 2_{-}, \mathrm{m} 3_{-}, \mathrm{m} 4 \_\right]=(\mathrm{mt}[\mathrm{m} 1, \mathrm{~m} 3] \times \mathrm{mt}[\mathrm{m} 2, \mathrm{~m} 4]-\mathrm{mt}[\mathrm{m} 1, \mathrm{~m} 4] \times \mathrm{mt}[\mathrm{m} 2, \mathrm{~m} 3]) / 2$

Outf $=1=\frac{1}{2}\left(g^{\mathrm{m} 1 \mathrm{~m} 3} g^{\mathrm{m} 2 \mathrm{~m} 4}-g^{\mathrm{m} 1 \mathrm{~m} 4} g^{\mathrm{m} 2 \mathrm{~m} 3}\right)$

$\ln [\circ]:=$

( ${ }^{*}$ Propagador do campo auxiliar $\mathrm{H}^{*}$ )

$\ln [\circ]:=\quad H H\left[\mathrm{~m} 1_{-}, \mathrm{n} 1_{-}, \mathrm{m} 2_{-}, \mathrm{n} 2_{-}\right]=2 \mathrm{I}$ IF $[\mathrm{m} 1, \mathrm{n} 1, \mathrm{~m} 2, \mathrm{n} 2]$

Outfo $=\tilde{a}\left(g^{\mathrm{m} 1 \mathrm{~m} 2} g^{\mathrm{n} 1 \mathrm{n} 2}-g^{\mathrm{m} 1 \mathrm{n} 2} g^{\mathrm{m} 2 \mathrm{n} 1}\right)$

$\ln [\circ]:=$

$\left({ }^{*}\right.$ Vértice AAH *)

$\ln [\circ]:=\quad H A A\left[m 11_{-}, \mathrm{m} 2_{-}, \mathrm{m} 3_{-}, \mathrm{m} 4 \_\right]=-\mathrm{I} / 2(\mathrm{mt}[\mathrm{m} 1, \mathrm{~m} 3] \times \mathrm{mt}[\mathrm{m} 2, \mathrm{~m} 4]-\mathrm{mt}[\mathrm{m} 1, \mathrm{~m} 4] \times \mathrm{mt}[\mathrm{m2}, \mathrm{m} 3])$

Out $\circ=-\frac{1}{2} \tilde{j}\left(g^{\mathrm{m} 1 \mathrm{~m} 3} g^{\mathrm{m} 2 \mathrm{~m} 4}-g^{\mathrm{m} 1 \mathrm{~m} 4} g^{\mathrm{m} 2 \mathrm{~m} 3}\right)$ 
2 | verificationHA.nb

\section{Definições para Passarino-Veltman}

$\left({ }^{\star *}\right.$ Integrais escalares usadas após a projeção na base tensorial $\left.{ }^{* *}\right)$

integrals $=\left\{1 / s p[p, p]^{\wedge} 2 / s p[q, q]^{\wedge} 2 \rightarrow \quad(3-d)(6-d) / s p[k, k]^{\wedge} 2 / c c\right.$,

$1 / s p[p, p]^{\wedge} 2 / s p[q, q] \rightarrow(3-d) / s p[k, k] / c c, 1 / s p[p, p] / s p[q, q]^{\wedge} 2 \rightarrow(3-d) / s p[k, k] / c c$,

$1 / \mathrm{sp}[\mathrm{p}, \mathrm{p}] / \mathrm{sp}[\mathrm{q}, \mathrm{q}] \rightarrow 1 / \mathrm{cc}\}$

Outf $\circ=\left\{\frac{1}{p^{4} q^{4}} \rightarrow \frac{(3-d)(6-d)}{\mathrm{cck} k^{4}}, \frac{1}{p^{4} q^{2}} \rightarrow \frac{3-d}{\mathrm{cck} k^{2}}, \frac{1}{p^{2} q^{4}} \rightarrow \frac{3-d}{\mathrm{cck} k^{2}}, \frac{1}{p^{2} q^{2}} \rightarrow \frac{1}{\mathrm{cc}}\right\}$

subs $=\{s p[k, p] \rightarrow(s p[q, q]-s p[p, p]-s p[k, k]) / 2, s p[k, q] \rightarrow(s p[q, q]+s p[k, k]-s p[p, p]) / 2$,

$s p[p, q] \rightarrow(s p[p, p]+s p[q, q]-s p[k, k]) / 2\}$

Outf $0=\left\{k \cdot p \rightarrow \frac{1}{2}\left(-k^{2}-p^{2}+q^{2}\right), k \cdot q \rightarrow \frac{1}{2}\left(k^{2}-p^{2}+q^{2}\right), p \cdot q \rightarrow \frac{1}{2}\left(-k^{2}+p^{2}+q^{2}\right)\right\}$

$\ln [\circ]:=$

$\left({ }^{* *}\right.$ Fator do resultado final $\left.{ }^{* *}\right)$

$\operatorname{In}[\circ]:=\quad f a c\left[d_{-}\right]=I^{\wedge}(d+1) * S p[k, k]^{\wedge}(d / 2-2)$ Gamma[2-d/2]Gamma[d/2-1 $]^{\wedge} 2 / 2^{\wedge} d / P j^{\wedge}(d / 2) / G a m m a[d-2]$

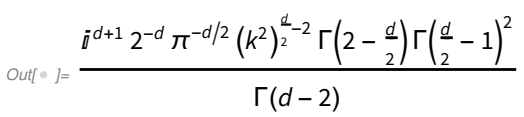

$n[\circ]:=$

$\left({ }^{* *}\right.$ Fator com $\left.d=4-2 \epsilon^{* \star}\right)$

$\left({ }^{* *} C_{-}\{Y M\}^{\wedge}\{a b\}=f^{\wedge}\{a \mid m\} f^{\wedge}\{b \mid m\}=C^{*} \delta^{\wedge}\left\{a b{ }^{* *}\right)\right.$

$\ln [\circ]:=\quad \operatorname{dn}\left[a_{-}, b_{-}\right]=g^{\wedge} 2 * C * \operatorname{SunDelta}[a, b] * \operatorname{Normal}[\operatorname{Series}[\operatorname{fac}[4-2 \epsilon],\{\epsilon, \odot,-1\}]]$

Out $\circ]=\frac{i C g^{2} \delta^{a b}}{16 \pi^{2} \epsilon}$

\section{Contribuição de um loop para o propagador HA}

\section{- Diagrama da Fig. 6.4 (Lado esquerdo)}

$\left({ }^{* *}\right.$ Integrando da integral devido ao loop do diagrama na Fig. $\left.6.4{ }^{* *}\right)$

$\left({ }^{* *}\right.$ Com o fator de simetria de $1 / 2{ }^{* *}$ )

int $=1 / 2 * H H[m 1, m 2, m 11, m 22] \times H A A[m 11, m 22, m, n] \times A A A[-k, q,-p, t t, r, s] \times A A[r, m, q] \times A A[s, n,-p] \times A A[t t, t, k]$

$\operatorname{In}[\circ]:=$ PropHAp[m1_, m2_, $\left.\mathrm{t}_{-}\right]=\% \quad / / \mathrm{Calc}$;

$\ln [\circ]:=$

$\left({ }^{* *}\right.$ Definindo a estrutura tensorial do resultado $\left.{ }^{* *}\right)$ 


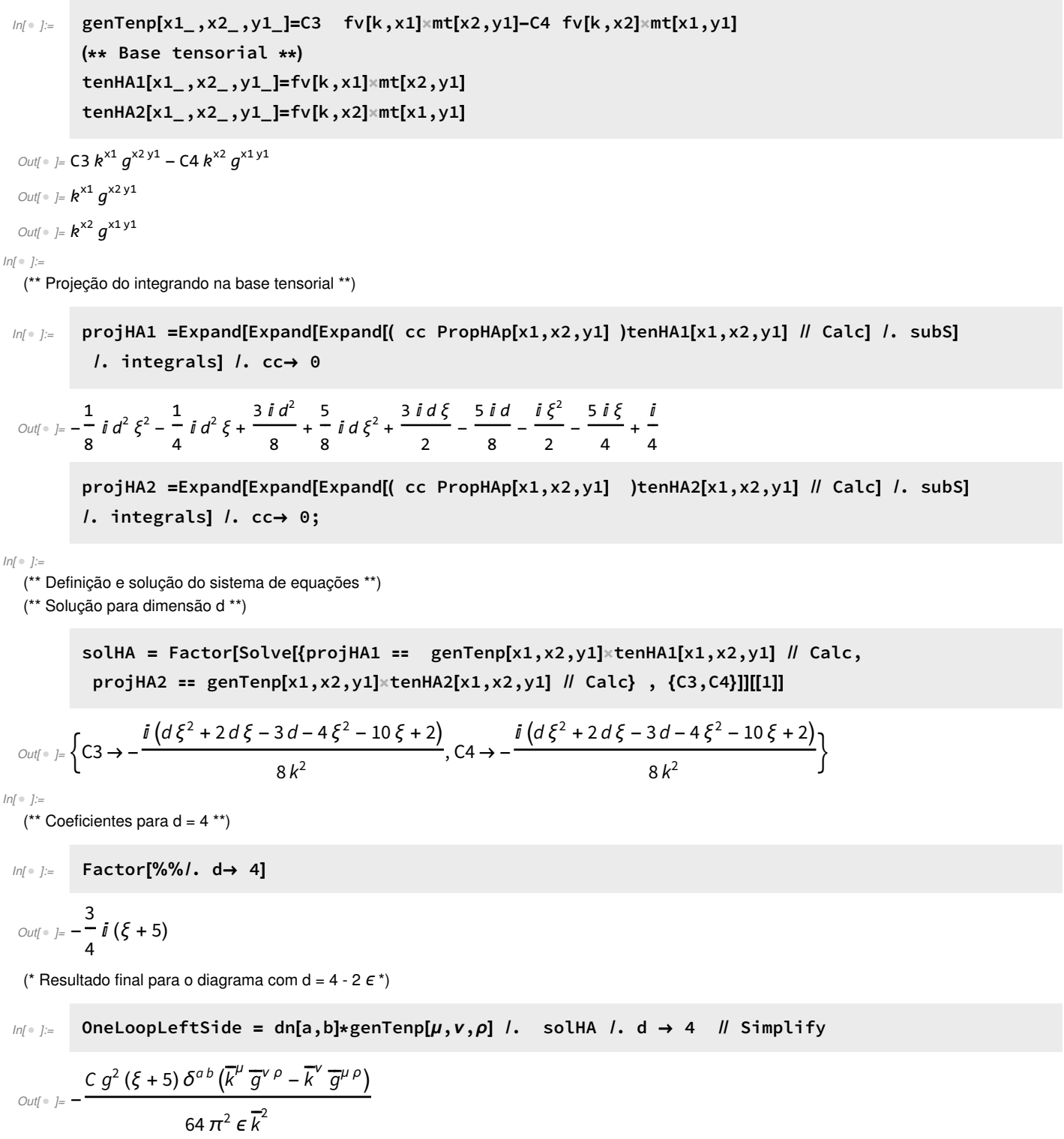

\section{Diagrama "Pinched"}

\section{- Diagrama 6.6(a) (Lado direito)}

$\left(^{* *}\right.$ Integrando da integral devido ao loop da Fig. 6.6(a) $\left.{ }^{* *}\right)$

$\left({ }^{* *}\right.$ Com fator de simetria $\left.=1{ }^{* \star}\right)$

int $=A A[m 1, m, q] \times A A[m 2, n, p] \times A A A[q,-p,-k, m, n, t t] \times A A[t t, t, k] / /$ Calc;

$(* * \mathrm{p}=\mathrm{q}-\mathrm{k} * *)$ 
4 | verificationHA.nb

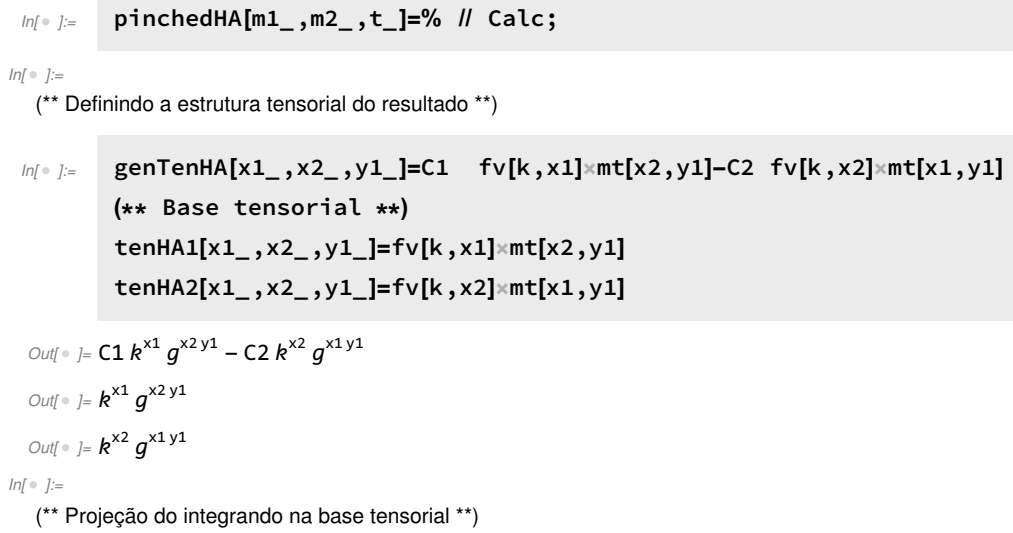

( ${ }^{* *}$ Projeção do integrando na base tensorial ${ }^{* *}$ )

projHA1 =Expand[Expand[Expand[( $\mathrm{cc}$ pinchedHA[x1,x2,y1] )tenHA1[x1,x2,y1] // Calc] /. subS]

I. integrals] $l . c c \rightarrow 0$

projHA2 $=$ Expand[Expand[Expand[( $c c$ pinchedHA[x1,x2,y1] )tenHA2[x1,x2,y1] // Calc] $/$. subS]

I. integrals] $l$. cc $\rightarrow 0$;

$\ln [\circ]:=$

( ${ }^{* *}$ Definição e solução do sistema de equações **)

$\left({ }^{* *}\right.$ Solução para dimensão $\left.{ }^{* *}\right)$

solPinchedHA = Factor[Solve[\{projHA1 == genTenHA[x1,x2,y1] tenHA1[x1, x2,y1] // Calc , projHA2 == genTenHA[x1, x2,y1] tenHA2[x1, x2,y1] // Calc $\},\{\mathrm{C} 1, \mathrm{C} 2\}]][[1]]$

Outf $\circ=\left\{C 1 \rightarrow-\frac{i\left(d \xi^{2}+2 d \xi-3 d-4 \xi^{2}-10 \xi+2\right)}{8 k^{2}}, C 2 \rightarrow-\frac{i\left(d \xi^{2}+2 d \xi-3 d-4 \xi^{2}-10 \xi+2\right)}{8 k^{2}}\right\}$

$\ln [\circ]:=$

$\left({ }^{* \star}\right.$ Coeficientes para $\left.d=4{ }^{* *}\right)$

$\ln [\circ]:=\quad$ Factor $[\% \% / . d \rightarrow 4]$

Out $\circ=-\frac{3}{4} i(\xi+5)$

$\left({ }^{*}\right.$ Resultado final para o diagrama com $\left.\mathrm{d}=4-2 \epsilon^{*}\right)$

$\operatorname{In}[\circ]:=\quad$ OneLoopRightSide $=\operatorname{dn}[\mathrm{a}, \mathrm{b}] * \operatorname{genTenHA}[\mu, \boldsymbol{v}, \rho] /$. solPinchedHA $/ . \mathrm{d} \rightarrow 4$ /I Simplify

Out $\circ=-\frac{C g^{2}(\xi+5) \delta^{a b}\left(\bar{k}^{\mu} \bar{g}^{\vee \rho}-\bar{k}^{v} \bar{g}^{\mu \rho}\right)}{64 \pi^{2} \in \bar{k}^{2}}$ 


\section{Verificação geral para um loop}

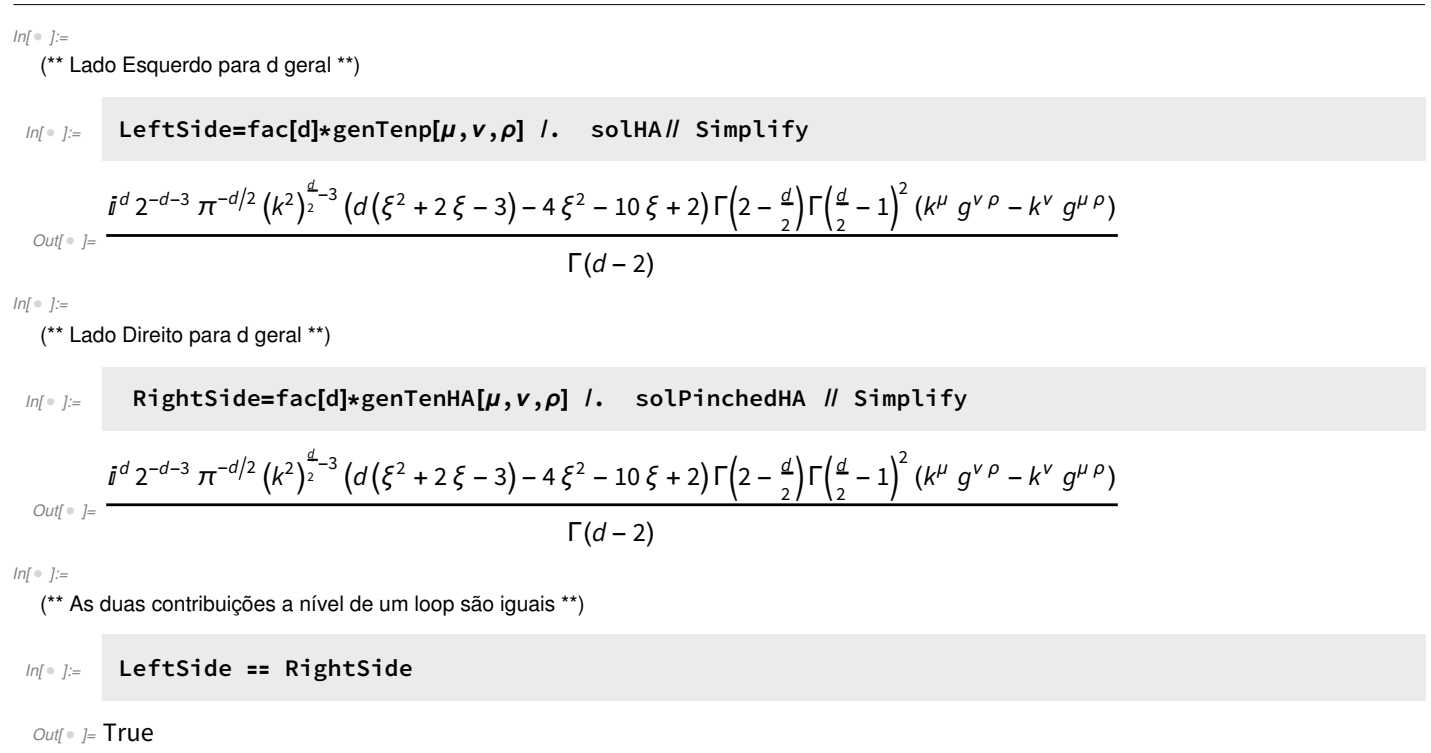




\section{APÊNDICE E}

\section{Resultados Gerais para Gravitação}

Neste apêndice deixamos como referência tabelas com os resultados obtidos para a verificação das identidades estruturais no contexto da gravitação.

\begin{tabular}{|c|c|c|c|}
\hline & 1 & $(\alpha-1)$ & $(\alpha-1)^{2}$ \\
\hline$C_{(1)}^{H \phi}$ & $\frac{1}{16(d-1)}$ & $\frac{1}{16(d-1)}$ & 0 \\
\hline$C_{(2)}^{H \phi}$ & $\frac{(d-2)(d+2)}{16(d-1)}$ & $-\frac{3 d^{2}-18 d+16}{16(d-1)}$ & $\frac{d^{3}-8 d^{2}+22 d-14}{16(d-1)}$ \\
\hline$C_{(3)}^{H \phi}$ & $-\frac{d^{2}+2 d+2}{32(d-2)(d-1)}$ & $\frac{7 d^{2}-41 d+32}{32(d-2)(d-1)}$ & $-\frac{5 d^{3}-37 d^{2}+96 d-60}{64(d-2)(d-1)}$ \\
\hline$C_{(4)}^{H \phi}$ & 0 & $\frac{1}{32(d-1)}$ & 0 \\
\hline$C_{(5)}^{H \phi}$ & $-\frac{d^{2}+d-1}{16(d-1)}$ & $-\frac{5-d}{8}$ & $-\frac{d-2}{8(d-1)}$ \\
\hline$C_{(6)}^{H \phi}$ & $\frac{d^{2}+6 d-4}{32(d-2)(d-1)}$ & $-\frac{d-5}{8(d-2)}$ & $-\frac{d-6}{32(d-1)}$ \\
\hline$C_{(7)}^{H \phi}$ & $-\frac{(d-2)^{2}}{32(d-1)}$ & $\frac{d-2}{16(d-1)}$ & $-\frac{(d-2)^{2}}{32(d-1)}$ \\
\hline$C_{(8)}^{H \phi}$ & $-\frac{(d-2)(d+2)}{16(d-1)}$ & $\frac{4 d^{2}-23 d+18}{16(d-1)}$ & $-\frac{d^{3}-8 d^{2}+21 d-12}{8(d-1)}$ \\
\hline$C_{(9)}^{H \phi}$ & $\frac{1}{16}(d+2)$ & $-\frac{4 d^{2}-23 d+22}{32(d-1)}$ & $\frac{d-2}{8(d-1)}$ \\
\hline$C_{(10)}^{H \phi}$ & 0 & $-\frac{(d-4) d}{16(d-1)}$ & $\frac{(d-4)^{2} d}{32(d-1)}$ \\
\hline$C_{(11)}^{H \phi}$ & $\frac{d^{2}-10 d+4}{32(d-1)}$ & $\frac{3\left(d^{2}-6 d+4\right)}{32(d-1)}$ & $-\frac{1}{64}(d-2)^{2}$ \\
\hline$C_{(12)}^{H \phi}$ & $-\frac{d}{8(d-2)(d-1)}$ & $-\frac{d-2}{32(d-1)}$ & $\frac{(d-4)^{2} d}{32(d-2)(d-1)}$ \\
\hline
\end{tabular}

Tabela E.1: Coeficientes da autoenergia mista $H \phi$, definida na Eq. (6.58), com unidades de $i \kappa k^{2} I_{U V}$. 


\begin{tabular}{ccccccccc}
\hline & 1 & $(\alpha-1)$ & $(\alpha-1)^{2}$ & & & 1 & $(\alpha-1)$ & $(\alpha-1)^{2}$ \\
\cline { 1 - 3 } \cline { 5 - 7 }$C_{(1)}^{H \phi}$ & $\frac{1}{48}$ & $\frac{1}{48}$ & 0 & & $C_{(7)}^{H \phi}$ & $-\frac{1}{24}$ & $\frac{1}{24}$ & $-\frac{1}{24}$ \\
$C_{(2)}^{H \phi}$ & $\frac{1}{4}$ & $\frac{1}{6}$ & $\frac{5}{24}$ & & $C_{(8)}^{H \phi}$ & $-\frac{1}{4}$ & $-\frac{5}{24}$ & $-\frac{1}{3}$ \\
$C_{(3)}^{H \phi}$ & $-\frac{13}{96}$ & $-\frac{5}{48}$ & $-\frac{13}{96}$ & & $C_{(9)}^{H \phi}$ & $\frac{3}{8}$ & $\frac{1}{16}$ & $\frac{1}{12}$ \\
$C_{(4)}^{H \phi}$ & 0 & $\frac{1}{96}$ & 0 & & $C_{(10)}^{H \phi}$ & 0 & 0 & 0 \\
$C_{(5)}^{H \phi}$ & $-\frac{19}{48}$ & $-\frac{1}{8}$ & $-\frac{1}{12}$ & & $C_{(11)}^{H \phi}$ & $-\frac{5}{24}$ & $-\frac{1}{8}$ & $-\frac{1}{16}$ \\
$C_{(6)}^{H \phi}$ & $\frac{3}{16}$ & $\frac{1}{16}$ & $\frac{1}{48}$ & & $C_{(12)}^{H \phi}$ & $-\frac{1}{12}$ & $-\frac{1}{48}$ & 0 \\
\hline
\end{tabular}

Tabela E.2: Polo UV dos coeficientes da autoenergia mista $H \phi$, definida na Eq. (6.58), com unidades de $i \kappa k^{2} I_{U V}$. 


\begin{tabular}{|c|c|c|c|}
\hline & 1 & $(\alpha-1)$ & $(\alpha-1)^{2}$ \\
\hline$C_{(1)}^{H H}$ & $-\frac{1}{2(d-2)}$ & $\frac{3(d-3) d}{16(d-2)(d-1)}$ & $-\frac{1}{4(d-1)(d+1)}$ \\
\hline$C_{(2)}^{H H}$ & $-\frac{d^{2}-2 d-2}{4(d-2)(d-1)}$ & $-\frac{4 d^{3}-17 d^{2}+31 d-32}{16(d-2)(d-1)}$ & $-\frac{d+2}{8(d-1)(d+1)}$ \\
\hline$C_{(3)}^{H H}$ & $\frac{1}{2(d-1)}$ & $\frac{d(3 d-5)}{16(d-2)(d-1)}$ & $\frac{d}{16(d-1)(d+1)}$ \\
\hline$C_{(4)}^{H H}$ & $\frac{d^{2}-2}{4(d-2)(d-1)}$ & $-\frac{4 d^{3}-23 d^{2}+33 d-16}{16(d-2)(d-1)}$ & $-\frac{1}{4(d-1)(d+1)}$ \\
\hline$C_{(5)}^{H H}$ & $-\frac{1}{(d-2)(d-1)}$ & $\frac{d^{2}-3 d-8}{8(d-2)(d-1)}$ & $\frac{d}{4(d-1)(d+1)}$ \\
\hline$C_{(6)}^{H H}$ & 0 & $-\frac{d-4}{2(d-1)}$ & $\frac{(d-4)(d-2) d}{4(d-1)(d+1)}$ \\
\hline$C_{(7)}^{H H}$ & 0 & $-\frac{(d-4)\left(d^{2}-31 d+24\right)}{16(d-1)}$ & $-\frac{(d-4)\left(4 d^{3}-16 d^{2}-3 d+14\right)}{4(d-1)(d+1)}$ \\
\hline$C_{(8)}^{H H}$ & 0 & $\frac{(d-4)^{2}(d-3)}{8(d-1)}$ & $-\frac{(d-4)(d-2)(d+2)}{4(d-1)(d+1)}$ \\
\hline$C_{(9)}^{H H}$ & 0 & $4-d$ & $\frac{(d-4)\left(4 d^{3}-17 d^{2}-4 d+20\right)}{8(d-1)(d+1)}$ \\
\hline$C_{(10)}^{H H}$ & $\frac{d^{2}-4 d+2}{2(d-2)(d-1)}$ & $\frac{3 d^{2}-12 d+8}{4(d-2)(d-1)}$ & $\frac{(d-2) d}{4(d-1)(d+1)}$ \\
\hline$C_{(11)}^{H H}$ & $\frac{d\left(d^{2}-d-4\right)}{4(d-2)(d-1)}$ & $\frac{d^{3}+15 d^{2}-50 d+16}{16(d-2)(d-1)}$ & $\frac{(d-2)(2 d+1)}{4(d-1)(d+1)}$ \\
\hline$C_{(12)}^{H H}$ & $\frac{d^{3}-2 d^{2}-4 d+4}{4(d-2)(d-1)}$ & $-\frac{(d-4)\left(d^{2}-4 d+2\right)}{4(d-2)(d-1)}$ & $\frac{(d-2) d}{4(d-1)(d+1)}$ \\
\hline$C_{(13)}^{H H}$ & $-\frac{(d-4) d}{2(d-2)(d-1)}$ & $\frac{3 d^{3}-23 d^{2}+50 d-16}{8(d-2)(d-1)}$ & $-\frac{(d-2)(d+2)}{4(d-1)(d+1)}$ \\
\hline$C_{(14)}^{H H}$ & $-\frac{d}{2(d-1)}$ & $\frac{d^{2}-11 d+12}{8(d-1)}$ & $-\frac{(d-2)(d+2)}{8(d-1)(d+1)}$ \\
\hline$C_{(15)}^{H H}$ & $\frac{d^{2}}{2(d-2)(d-1)}$ & $-\frac{3 d^{3}-15 d^{2}+18 d-16}{8(d-2)(d-1)}$ & $-\frac{d-2}{2(d-1)(d+1)}$ \\
\hline$C_{(16)}^{H H}$ & $\frac{2 d^{2}-d-2}{4(d-1)}$ & $-\frac{11 d^{2}-89 d+72}{16(d-1)}$ & $\frac{4 d^{4}-28 d^{3}+55 d^{2}+32 d-52}{8(d-1)(d+1)}$ \\
\hline$C_{(17)}^{H H}$ & $\frac{d}{2(d-1)}$ & $-\frac{(d-7) d}{8(d-1)}$ & $\frac{(d-2) d}{4(d-1)(d+1)}$ \\
\hline$C_{(18)}^{H H}$ & $-\frac{2 d^{2}+d-2}{2(d-1)}$ & $\frac{11 d^{2}-73 d+72}{8(d-1)}$ & $-\frac{(d-2)(2 d+3)}{2(d-1)(d+1)}$ \\
\hline$C_{(19)}^{H H}$ & -1 & -1 & $-\frac{(d-2)(d+2)}{4(d-1)(d+1)}$ \\
\hline$C_{(20)}^{H H}$ & 0 & 0 & $\frac{(d-6)(d-4)(d-2) d}{16(d-1)(d+1)}$ \\
\hline$C_{(21)}^{H H}$ & 0 & $-\frac{(d-4)(d-3) d}{16(d-1)}$ & $\frac{(d-4)(d-2) d}{16(d-1)(d+1)}$ \\
\hline$C_{(22)}^{H H}$ & 1 & $\frac{3 d-4}{4(d-1)}$ & $\frac{8 d^{3}-23 d^{2}-10 d+24}{16(d-1)(d+1)}$ \\
\hline
\end{tabular}

Tabela E.3: Coeficientes da autoenergia mista $H H$, definida na Eq. (6.62), com unidades de $k^{2} I_{11}$. 


\begin{tabular}{cccc}
\hline & 1 & $(\alpha-1)$ & $(\alpha-1)^{2}$ \\
\hline$C_{(1)}^{H H}$ & $-\frac{1}{4}$ & $\frac{1}{8}$ & $-\frac{1}{60}$ \\
$C_{(2)}^{H H}$ & $-\frac{1}{4}$ & $-\frac{19}{24}$ & $-\frac{1}{20}$ \\
$C_{(3)}^{H H}$ & $\frac{1}{6}$ & $\frac{7}{24}$ & $\frac{1}{60}$ \\
$C_{(4)}^{H H}$ & $\frac{7}{12}$ & $-\frac{1}{24}$ & $-\frac{1}{60}$ \\
$C_{(5)}^{H H}$ & $-\frac{1}{6}$ & $-\frac{1}{12}$ & $\frac{1}{15}$ \\
$C_{(6)}^{H H}$ & 0 & 0 & 0 \\
$C_{(7)}^{H H}$ & 0 & 0 & 0 \\
$C_{(8)}^{H H}$ & 0 & 0 & 0 \\
$C_{(9)}^{H H}$ & 0 & 0 & 0 \\
$C_{(10)}^{H H}$ & $\frac{1}{6}$ & $\frac{1}{3}$ & $\frac{2}{15}$ \\
$C_{(11)}^{H H}$ & $\frac{4}{3}$ & $\frac{5}{4}$ & $\frac{3}{10}$ \\
\hline
\end{tabular}

\begin{tabular}{cccc}
\hline & 1 & $(\alpha-1)$ & $(\alpha-1)^{2}$ \\
\hline$C_{(12)}^{H H}$ & $\frac{5}{6}$ & 0 & $\frac{2}{15}$
\end{tabular}

$C_{(13)}^{H H} \quad 0 \quad \frac{1}{6} \quad-\frac{1}{5}$$$
C_{(14)}^{H H} \quad-\frac{2}{3} \quad-\frac{2}{3} \quad-\frac{1}{10}
$$$$
C_{(15)}^{H H} \quad \frac{4}{3} \quad-\frac{1}{6} \quad-\frac{1}{15}
$$$$
\begin{array}{llll}
C_{(16)}^{H H} & \frac{13}{6} & \frac{9}{4} & \frac{47}{30}
\end{array}
$$$$
\begin{array}{llll}
C_{(17)}^{H H} & \frac{2}{3} & \frac{1}{2} & \frac{2}{15}
\end{array}
$$$$
C_{(18)}^{H H} \quad-\frac{17}{3} \quad-\frac{11}{6} \quad-\frac{11}{15}
$$$$
\begin{array}{llll}
C_{(19)}^{H H} & -1 & -1 & -\frac{1}{5}
\end{array}
$$$$
\begin{array}{llll}
C_{(20)}^{H H} & 0 & 0 & 0
\end{array}
$$$$
\begin{array}{llll}
C_{(21)}^{H H} & 0 & 0 & 0
\end{array}
$$$$
\begin{array}{llll}
C_{(22)}^{H H} & 1 & \frac{2}{3} & \frac{8}{15}
\end{array}
$$

Tabela E.4: Polo UV dos coeficientes da autoenergia mista $H H$, definida na Eq. (6.62), com unidades de $k^{2} I_{U V}$. 


\begin{tabular}{|c|c|c|c|}
\hline & 1 & $(\alpha-1)$ & $(\alpha-1)^{2}$ \\
\hline$P_{(1)}^{H \phi}$ & 0 & $-\frac{i}{16(d-1)}$ & $-\frac{i}{16(d-1)}$ \\
\hline$P_{(2)}^{H \phi}$ & $\frac{i\left(2 d^{2}+d-5\right)}{16(d-1)}$ & $-\frac{i(5(d-6) d+26)}{16(d-1)}$ & $\frac{i(d((d-8) d+24)-18)}{16(d-1)}$ \\
\hline$P_{(3)}^{H \phi}$ & $-\frac{i(d((d-5) d+19)-6)}{32(d-2)(d-1)}$ & $\frac{i\left(23 d^{2}-147 d+122\right)}{64\left(d^{2}-3 d+2\right)}$ & $-\frac{i(d(d(10 d-73)+193)-134)}{64(d-2)(d-1)}$ \\
\hline$P_{(4)}^{H \phi}$ & $-\frac{i}{16(d-1)}$ & $-\frac{3 i}{32(d-1)}$ & $-\frac{i}{32(d-1)}$ \\
\hline$P_{(5)}^{H \phi}$ & $-\frac{i\left(d^{2}-4\right)}{8(d-1)}$ & $\frac{i(3(d-6) d+16)}{8(d-1)}$ & $-\frac{i(d((d-8) d+22)-14)}{8(d-1)}$ \\
\hline$P_{(6)}^{H \phi}$ & $\frac{i((d-6) d-3)}{16(d-1)}$ & $\frac{i(4 d-27)}{32}$ & $-\frac{i(d((d-7) d+21)-17)}{32(d-1)}$ \\
\hline$P_{(7)}^{H \phi}$ & $-\frac{i((d-10) d+4)}{16(d-1)}$ & $-\frac{i(d(3 d-26)+26)}{16(d-1)}$ & $\frac{i(d((d-9) d+34)-28)}{32(d-1)}$ \\
\hline$P_{(8)}^{H \phi}$ & $-\frac{i(d+2)}{8}$ & $\frac{i(d(4 d-23)+24)}{16(d-1)}$ & $-\frac{i(3 d-10)}{16(d-1)}$ \\
\hline$P_{(9)}^{H \phi}$ & $\frac{i(d+2)(2 d-3)}{16(d-1)}$ & $-\frac{i(d(12 d-71)+54)}{32(d-1)}$ & $\frac{i(d(4(d-8) d+85)-50)}{32(d-1)}$ \\
\hline$P_{(10)}^{H \phi}$ & 0 & $\frac{i(d-6) d}{16(d-1)}$ & $-\frac{i((d-5)(d-4) d+4)}{32(d-1)}$ \\
\hline$P_{(11)}^{H \phi}$ & $\frac{i(d((d-5) d+28)-20)}{32(d-2)(d-1)}$ & $-\frac{i\left(7 d^{2}-48 d+44\right)}{16\left(d^{2}-3 d+2\right)}$ & $\frac{i(d(d(5 d-39)+110)-80)}{32(d-2)(d-1)}$ \\
\hline$P_{(12)}^{H \phi}$ & $-\frac{i(d-2)}{32(d-1)}$ & $\frac{i((d-9) d+2)}{64(d-1)}$ & $-\frac{i(d(d(2 d-15)+43)-34)}{64(d-1)}$ \\
\hline
\end{tabular}

Tabela E.5: Coeficientes do propagador $H \phi$, definido na Eq. (6.59), com unidades de $\kappa^{3} I_{11}$. 


\begin{tabular}{cccc}
\hline & 1 & $(\alpha-1)$ & $(\alpha-1)^{2}$ \\
\hline$P_{(1)}^{H \phi}$ & 0 & $-\frac{i}{48}$ & $-\frac{i}{48}$ \\
$P_{(2)}^{H \phi}$ & $\frac{31 i}{48}$ & $\frac{7 i}{24}$ & $\frac{7 i}{24}$ \\
$P_{(3)}^{H \phi}$ & $-\frac{9 i}{32}$ & $-\frac{49 i}{192}$ & $-\frac{55 i}{192}$ \\
$P_{(4)}^{H \phi}$ & $-\frac{i}{48}$ & $-\frac{i}{32}$ & $-\frac{i}{96}$ \\
$P_{(5)}^{H \phi}$ & $-\frac{i}{2}$ & $-\frac{i}{3}$ & $-\frac{5 i}{12}$ \\
$P_{(6)}^{H \phi}$ & $-\frac{11 i}{48}$ & $-\frac{11 i}{32}$ & $-\frac{19 i}{96}$ \\
$P_{(7)}^{H \phi}$ & $\frac{5 i}{12}$ & $\frac{5 i}{8}$ & $\frac{7 i}{24}$ \\
$P_{(8)}^{H \phi}$ & $-\frac{3 i}{4}$ & $-\frac{i}{12}$ & $-\frac{i}{24}$ \\
$P_{(9)}^{H \phi}$ & $\frac{5 i}{8}$ & $\frac{19 i}{48}$ & $\frac{17 i}{48}$ \\
$P_{(10)}^{H \phi}$ & 0 & $-\frac{i}{6}$ & $-\frac{i}{24}$ \\
$P_{(11)}^{H \phi}$ & $\frac{19 i}{48}$ & $\frac{3 i}{8}$ & $\frac{7 i}{24}$ \\
$P_{(12)}^{H \phi}$ & $-\frac{i}{48}$ & $-\frac{3 i}{32}$ & $-\frac{13 i}{96}$ \\
\hline
\end{tabular}

Tabela E.6: Polo UV dos coeficientes do propagador $H \phi$, definido na Eq. (6.59), com unidades de $\kappa^{3} I_{U V}$. 


\begin{tabular}{|c|c|c|c|}
\hline & 1 & $(\alpha-1)$ & $(\alpha-1)^{2}$ \\
\hline$P_{(1)}^{H H}$ & $-\frac{1}{2(d-1)}$ & $-\frac{d(3 d-5)}{16(d-2)(d-1)}$ & $-\frac{d}{16(d-1)(d+1)}$ \\
\hline$P_{(2)}^{H H}$ & $\frac{2 d^{2}-3 d-4}{8(d-2)(d-1)}$ & $\frac{2 d^{3}-7 d^{2}+15 d-20}{16(d-2)(d-1)}$ & $\frac{3 d+4}{32(d-1)(d+1)}$ \\
\hline$P_{(3)}^{H H}$ & $-\frac{1}{8(d-2)(d-1)}$ & $-\frac{d^{2}-2 d+4}{16(d-2)(d-1)}$ & $\frac{3 d+4}{64(d-1)(d+1)}$ \\
\hline$P_{(4)}^{H H}$ & $-\frac{d+1}{4(d-1)}$ & $-\frac{3 d^{2}-d-8}{16(d-2)(d-1)}$ & $-\frac{d}{16(d-1)(d+1)}$ \\
\hline$P_{(5)}^{H H}$ & $\frac{1}{2(d-2)}$ & $\frac{d^{2}-d+4}{8(d-2)(d-1)}$ & $-\frac{d}{16(d-1)(d+1)}$ \\
\hline$P_{(6)}^{H H}$ & 0 & $-\frac{4-d}{2}$ & $-\frac{(d-4)\left(4 d^{3}-17 d^{2}-4 d+20\right)}{16(d-1)(d+1)}$ \\
\hline$P_{(7)}^{H H}$ & 0 & $\frac{(d-4)(d-3) d}{16(d-1)}$ & $-\frac{(d-4)(d-2) d}{16(d-1)(d+1)}$ \\
\hline$P_{(8)}^{H H}$ & 0 & $-\frac{(d-4)(d-3)(d-2)}{8(d-1)}$ & $\frac{(d-4)(d-2)(3 d+4)}{16(d-1)(d+1)}$ \\
\hline$P_{(9)}^{H H}$ & 0 & $-\frac{(d-4)(d+2)}{8(d-2)(d-1)}$ & $\frac{(d-4)\left(5 d^{3}-14 d^{2}-20 d+8\right)}{32(d-2)(d-1)(d+1)}$ \\
\hline$P_{(10)}^{H H}$ & -1 & $-\frac{3 d-4}{4(d-1)}$ & $-\frac{8 d^{3}-23 d^{2}-10 d+24}{16(d-1)(d+1)}$ \\
\hline$P_{(11)}^{H H}$ & $-\frac{d^{2}}{4(d-1)}$ & $\frac{3 d^{2}-41 d+32}{16(d-1)}$ & $-\frac{4 d^{4}-28 d^{3}+57 d^{2}+30 d-56}{16(d-1)(d+1)}$ \\
\hline$P_{(12)}^{H H}$ & $-\frac{(d-2)(d+1)}{4(d-1)}$ & $-\frac{5-d}{2}$ & $-\frac{4 d^{4}-28 d^{3}+53 d^{2}+34 d-48}{16(d-1)(d+1)}$ \\
\hline$P_{(13)}^{H H}$ & $-\frac{d}{2(d-1)}$ & $\frac{d^{2}-9 d+6}{8(d-1)}$ & $-\frac{(d-2)(3 d+2)}{16(d-1)(d+1)}$ \\
\hline$P_{(14)}^{H H}$ & 0 & $-\frac{1-d}{8}$ & $\frac{(d-2)(d+2)}{32(d-1)(d+1)}$ \\
\hline$P_{(15)}^{H H}$ & $\frac{d}{2(d-1)}$ & $-\frac{d^{2}-11 d+12}{8(d-1)}$ & $\frac{(d-2)(d+2)}{8(d-1)(d+1)}$ \\
\hline$P_{(16)}^{H H}$ & $-\frac{4 d^{3}-7 d^{2}-8 d+8}{8(d-2)(d-1)}$ & $\frac{9 d^{3}-87 d^{2}+202 d-120}{16(d-2)(d-1)}$ & $-\frac{4 d^{4}-28 d^{3}+69 d^{2}+18 d-80}{32(d-1)(d+1)}$ \\
\hline$P_{(17)}^{H H}$ & $-\frac{d}{2(d-2)(d-1)}$ & $-\frac{d^{3}-5 d^{2}+8 d+4}{8(d-2)(d-1)}$ & $\frac{(d-2)(d+2)}{16(d-1)(d+1)}$ \\
\hline$P_{(18)}^{H H}$ & $d+1$ & $-\frac{11 d^{2}-81 d+72}{8(d-1)}$ & $\frac{4 d^{4}-28 d^{3}+59 d^{2}+30 d-64}{8(d-1)(d+1)}$ \\
\hline$P_{(19)}^{H H}$ & $-\frac{3}{d-2}$ & $\frac{7 d^{2}-44 d+36}{4(d-2)(d-1)}$ & $-\frac{3\left(4 d^{4}-25 d^{3}+44 d^{2}+28 d-48\right)}{16(d-2)(d-1)(d+1)}$ \\
\hline$P_{(20)}^{H H}$ & 0 & 0 & $-\frac{(d-6)(d-4)(d-2) d}{64(d-1)(d+1)}$ \\
\hline$P_{(21)}^{H H}$ & 0 & $\frac{(d-4)\left(d^{2}-12 d+12\right)}{16(d-1)}$ & $\frac{(d-4)\left(16 d^{3}-69 d^{2}-10 d+72\right)}{64(d-1)(d+1)}$ \\
\hline$P_{(22)}^{H H}$ & $\frac{d\left(2 d^{2}+d-24\right)+20}{8(d-2)^{2}(d-1)}$ & $-\frac{d(d(5 d-49)+130)-88}{8(d-2)^{2}(d-1)}$ & $-\frac{d(d(d((97-8 d) d-390)+468)+360)-576}{64(d-2)^{2}\left(d^{2}-1\right)}$ \\
\hline
\end{tabular}

Tabela E.7: Coeficientes do propagador $H H$, definido na Eq. (6.63), com unidades de $\kappa^{4} k^{2} I_{11}$. 


\begin{tabular}{cccc}
\hline & 1 & $(\alpha-1)$ & $(\alpha-1)^{2}$ \\
\hline$P_{(1)}^{H H}$ & $-\frac{1}{6}$ & $-\frac{7}{24}$ & $-\frac{1}{60}$ \\
$P_{(2)}^{H H}$ & $\frac{1}{3}$ & $\frac{7}{12}$ & $\frac{1}{30}$ \\
$P_{(3)}^{H H}$ & $-\frac{1}{48}$ & $-\frac{1}{8}$ & $\frac{1}{60}$ \\
$P_{(4)}^{H H}$ & $-\frac{5}{12}$ & $-\frac{3}{8}$ & $-\frac{1}{60}$ \\
$P_{(5)}^{H H}$ & $\frac{1}{4}$ & $\frac{1}{3}$ & $-\frac{1}{60}$ \\
$P_{(6)}^{H H}$ & 0 & 0 & 0 \\
$P_{(7)}^{H H}$ & 0 & 0 & 0 \\
$P_{(8)}^{H H}$ & 0 & 0 & 0 \\
$P_{(9)}^{H H}$ & 0 & 0 & 0 \\
$P_{(10)}^{H H}$ & -1 & $-\frac{2}{3}$ & $-\frac{8}{15}$ \\
$P_{(11)}^{H H}$ & $-\frac{4}{3}$ & $-\frac{7}{4}$ & $-\frac{13}{15}$ \\
\hline & & & \\
\hline
\end{tabular}

\begin{tabular}{cccc}
\hline & 1 & $(\alpha-1)$ & $(\alpha-1)^{2}$ \\
\hline$P_{(12)}^{H H}$ & $-\frac{5}{6}$ & $-\frac{1}{2}$ & $-\frac{7}{10}$
\end{tabular}

$$
\begin{array}{llll}
P_{(13)}^{H H} & -\frac{2}{3} & -\frac{7}{12} & -\frac{7}{60}
\end{array}
$$$$
\begin{array}{llll}
P_{(14)}^{H H} & 0 & \frac{3}{8} & \frac{1}{40}
\end{array}
$$$$
\begin{array}{llll}
P_{(15)}^{H H} & \frac{2}{3} & \frac{2}{3} & \frac{1}{10}
\end{array}
$$$$
P_{(16)}^{H H} \quad-\frac{5}{2} \quad-\frac{4}{3} \quad-\frac{41}{60}
$$$$
\begin{array}{llll}
P_{(17)}^{H H} & -\frac{1}{3} & -\frac{5}{12} & \frac{1}{20}
\end{array}
$$$$
\begin{array}{llll}
P_{(18)}^{H H} & 5 & \frac{19}{6} & \frac{29}{15}
\end{array}
$$$$
P_{(19)}^{H H} \quad-\frac{3}{2} \quad-\frac{7}{6} \quad-\frac{6}{5}
$$

$\begin{array}{llll}P_{(20)}^{H H} & 0 & 0 & 0\end{array}$

$\begin{array}{llll}P_{(21)}^{H H} & 0 & 0 & 0\end{array}$

$P_{(22)}^{H H} \quad \frac{17}{24} \quad \frac{1}{3} \quad-\frac{1}{120}$

Tabela E.8: Polo UV dos coeficientes do propagador $H H$, definido na Eq. (6.63), com unidades de $\kappa^{4} k^{2} I_{U V}$. 


\section{APENNDICE F}

\section{Amplitude $(A A A A)$ nA TeOria DE YAng-Mills}

Aqui apresentamos o código usado para verificar a igualdade das amplitudes (off-shell) que contribuem para o espalhamento glúon-glúon ${ }^{1}$ nos formalismos de primeira e segunda ordem conforme mencionado na página 10. Mostraremos também os diagramas (ordem árvore) das contribuições para a amplitude $\left(A_{\mu_{1}}^{m_{1}}\left(p_{1}\right) A_{\mu_{2}}^{m_{2}}\left(p_{2}\right) A_{\mu_{3}}^{m_{3}}\left(p_{3}\right) A_{\mu_{4}}^{m_{4}}\left(p_{4}\right)\right)$ que foram computados usando computação simbólica.

Evidentemente as amplitudes físicas (on-shell, considerando a polarização) que contribuem para o espalhamento glúon-glúon devem ser iguais ${ }^{2}$ em ambos formalismos, mas além disso verifica-se que a função de Green conexa de 4-pontos, a nível de árvore, também é igual nos dois formalismos, conforme a Eq. (3.15).

\section{F.1 Formalismo de segunda ordem}

Na Fig. F.1 estão os diagramas que contribuem para a função de Green conexa de 4-pontos

$$
\left\langle 0\left|T A_{\mu_{1}}^{m_{1}}\left(p_{1}\right) A_{\mu_{2}}^{m_{2}}\left(p_{2}\right) A_{\mu_{3}}^{m_{3}}\left(p_{3}\right) A_{\mu_{4}}^{m_{4}}\left(p_{4}\right)\right| 0\right\rangle
$$

no formalismo de segunda ordem usando as regras de Feynman obtidas no apêndice C.

Na Fig. F.2 mostramos os canais s, t e u do espalhamento glúon-glúon.

\section{F.2 Formalismo de primeira ordem}

No formalismo de primeira ordem podemos classificar as contribuições para a função de Green conexa de 4-pontos $\left\langle 0\left|T A_{\mu_{1}}^{m_{1}}\left(p_{1}\right) A_{\mu_{2}}^{m_{2}}\left(p_{2}\right) A_{\mu_{3}}^{m_{3}}\left(p_{3}\right) A_{\mu_{4}}^{m_{4}}\left(p_{4}\right)\right| 0\right\rangle$ no nível árvore em três grupos distintos:

\footnotetext{
${ }^{1}$ A demonstração vale para qualquer campo de calibre do $\mathrm{SU}(\mathrm{N})$, ou seja, vale para partículas além dos glúons (relativa a $N=3$ ). No entanto, para facilitar a nomenclatura usamos o termo glúon para designar a partícula associada a um campo de calibre qualquer da Teoria de YM com grupo $S U(N)$.

${ }^{2}$ Já que os formalismos são obtidos por redefinições locais do campo auxiliar.
} 

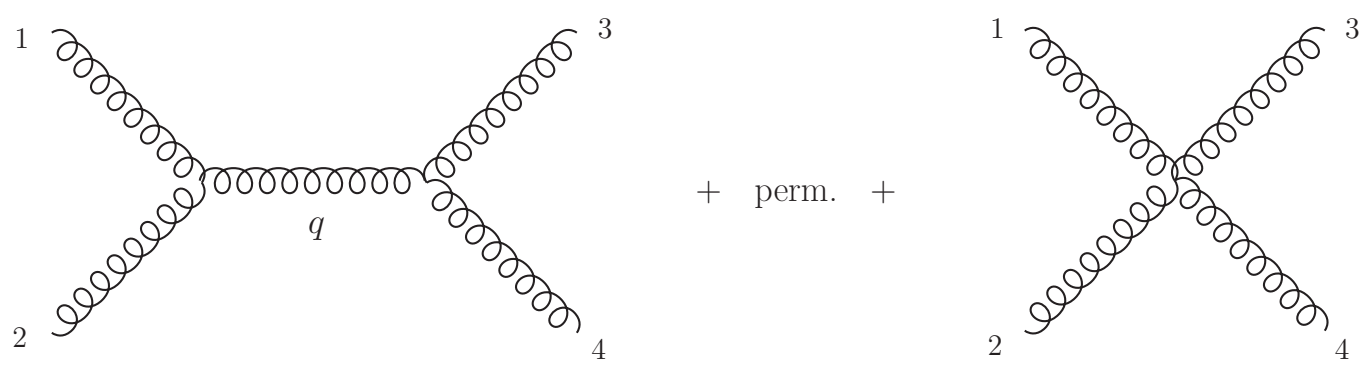

Figura F.1: Diagramas de ordem árvore do espalhamento glúon-glúon. Os índices $i$ representam o trio $\left(p_{i}, \mu_{i}, m_{i}\right)$, o momento interno do canal s é dado por $q=p_{1}+p_{2}=-\left(p_{3}+p_{4}\right)$. Por "perm." estamos nos referindo as permutações do primeiro diagrama (canal s): canais t e u.

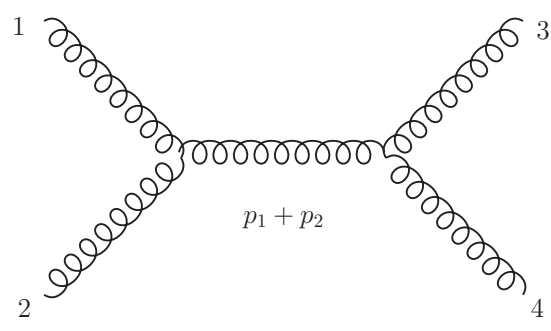

canal s

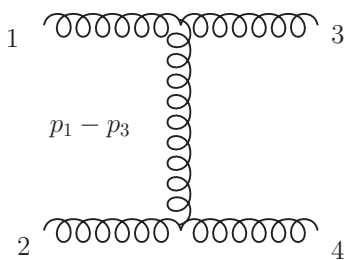

canal t

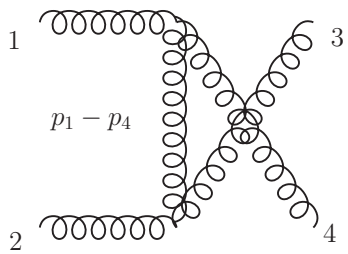

canal $\mathrm{u}$

Figura F.2: Canais s, t e u do espalhamento glúon-glúon no formalismo de segunda ordem.

- Tipo 0: sem pernas externas mistas.

- Tipo I: uma perna externa mista.

- Tipo II: duas pernas externas mistas.

Nas Figs. F.3-5 temos os diagramas (canais s) que contribuem no nível de árvore para a função de Green conexa de 4-pontos $\left\langle 0\left|T A_{\mu_{1}}^{m_{1}}\left(p_{1}\right) A_{\mu_{2}}^{m_{2}}\left(p_{2}\right) A_{\mu_{3}}^{m_{3}}\left(p_{3}\right) A_{\mu_{4}}^{m_{4}}\left(p_{4}\right)\right| 0\right\rangle$, respectivamente, do tipo 0, I e II. Note que os índices foram omitidos, pois as pernas externas seguem o mesmo padrão dos diagramas da Fig. F.1.

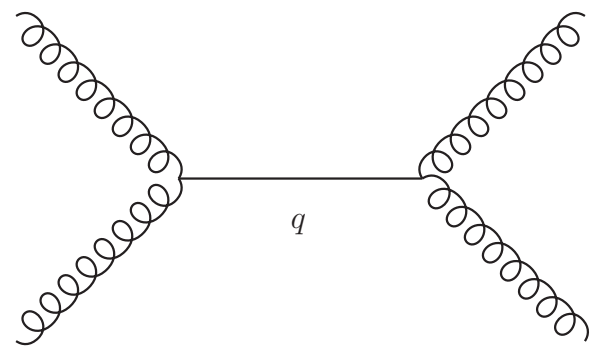

Figura F.3: Diagrama (canal s) do tipo 0. O momento interno $q=p_{1}+p_{2}=-\left(p_{3}+p_{4}\right)$. 

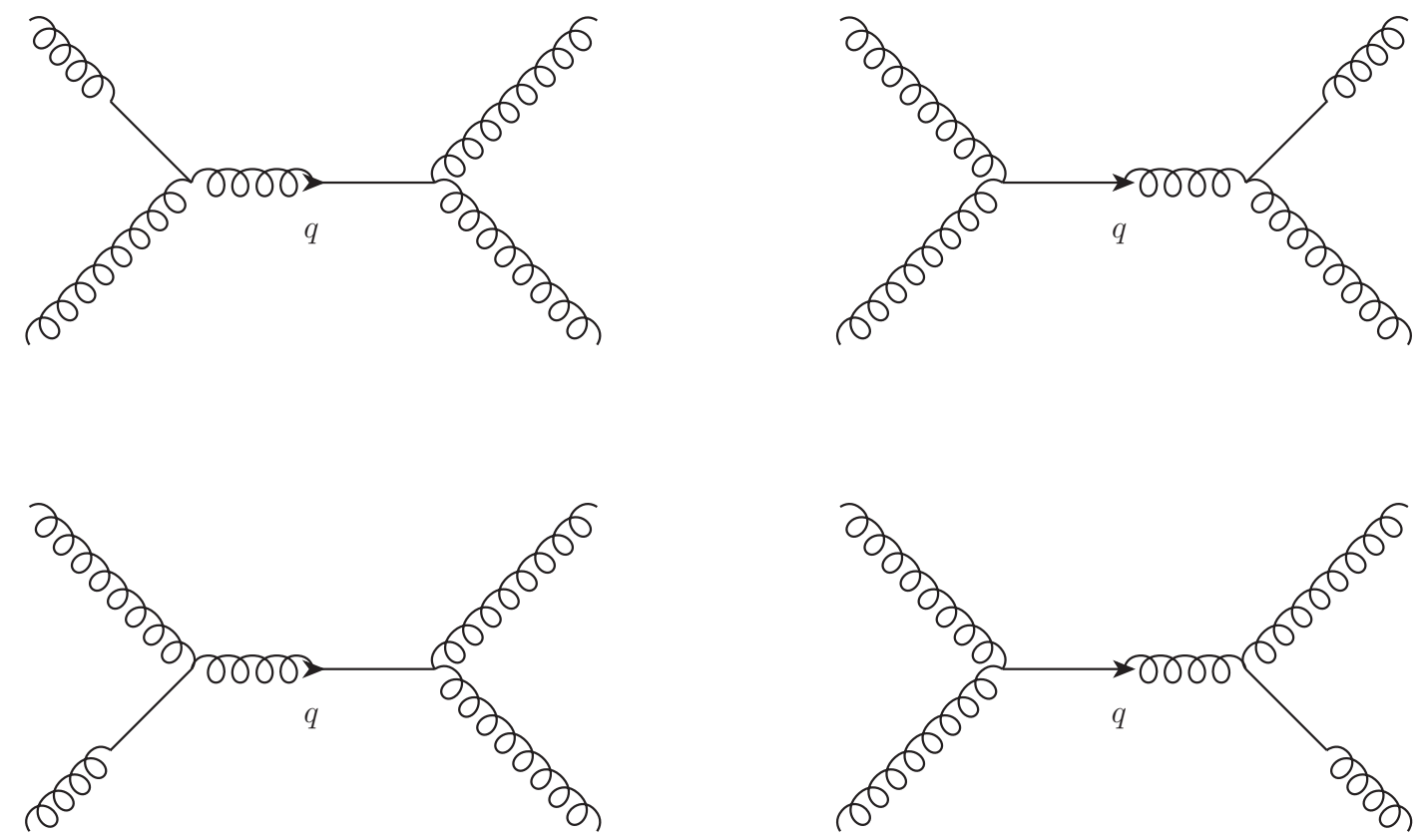

Figura F.4: Diagramas (canal s) do tipo I. O momento interno $q=p_{1}+p_{2}=-\left(p_{3}+p_{4}\right)$.
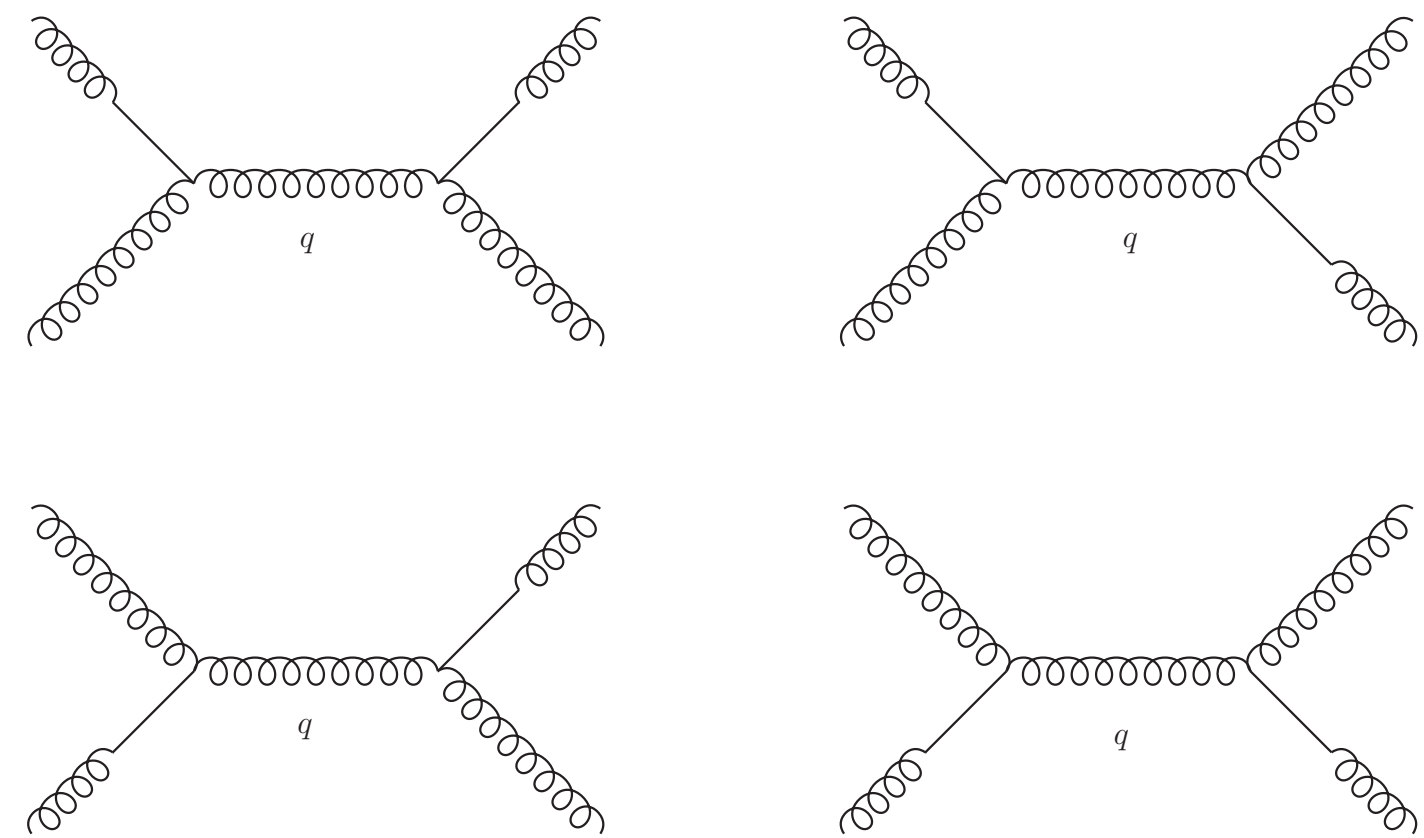

Figura F.5: Diagramas (canal s) do tipo II. O momento interno $q=p_{1}+p_{2}=-\left(p_{3}+p_{4}\right)$.

\section{F.3 Código do Mathematica 12}

A omissão dos canais t e u não é de fato revelante uma vez que a equivalência entre os diagramas no formalismos de primeira e segunda ordem pode ser verificada usando somente os diagramas relativos ao canal s. Isso se deve a presença dos fatores de cor; como cada diagrama é proporcional a um fator de cor como $f^{m_{1} m_{2} a} f^{m_{4} m_{3} a}$, sendo este o caso dos diagramas que mostramos nas 
figuras desse apêndice (exceto a Fig. F.2), podemos considerá-los separadamente ${ }^{3}$.

A única exceção é o vértice quártico que é a soma de três contribuições proporcionais a fatores de cor distintos (sendo um deles o mencionado anteriormente), note que cada fator de cor no vértice quártico é relativo a um canal s, t ou u. Por isso para verificação da função de Green $\left\langle 0\left|T A_{\mu_{1}}^{m_{1}}\left(p_{1}\right) A_{\mu_{2}}^{m_{2}}\left(p_{2}\right) A_{\mu_{3}}^{m_{3}}\left(p_{3}\right) A_{\mu_{4}}^{m_{4}}\left(p_{4}\right)\right| 0\right\rangle$ simplesmente comparamos a soma dos diagramas da Fig. F.3-5 com a soma do primeiro diagrama da Fig. F.1 e a parte do vértice quártico proporcional ao fator de cor do canal s.

O fator de cor de referência (do canal s) é igual a $f^{m_{1} m_{2} a} f^{m_{4} m_{3} a}$, sendo que no código abaixo todos os diagramas computados são proporcionais a tal fator ${ }^{4}$.

\section{F.4 Discussão dos resultados}

Como vemos abaixo para verificar a equivalência da função de Green conexa de 4-pontos não foi necessário usar a conservação de momento nos vértice, isso é, consideramos que $q$ (momento interno) era uma variável independente. Esse resultado não é algo muito surpreendente já que as funções de Green não são quantidades físicas e podem estar sujeitas a condições não físicas como partículas externas fora da camada de massa ou dependerem do parâmetro de calibre.

No entanto, no caso da amplitude, obtida pela amputação da função de Green pelo inverso do propagador do campo de calibre, é necessário considerar a conservação de momento. Ou seja, só verifica-se a equivalência entre as amplitudes (off-shell ou on-shell) do espalhamento glúon-glúon quando usamos $q=p_{1}+p_{2}=-\left(p_{3}+p_{4}\right)$.

No caso das amplitudes físicas em que consideramos os glúons externos na camada de massa e satisfazendo condições de transversalidade $\left(\varepsilon_{\mu} p^{\mu}=0\right)$, devido a polarização dos glúons, a dependência no parâmetro de calibre $\xi$ da soma dos diagramas do tipo II se cancela ${ }^{5}$. Embora que não mostremos esse resultado no código abaixo, tal cancelamento é esperado devido a equivalência entre as amplitudes off-shell computadas nos dois formalismos.

Sendo uma quantidade física tais amplitudes não poderiam depender do calibre. Logo, isso não só colocou em teste a consistência interna dos cálculos realizados no formalismo de primeira ordem como também dos resultados para a amplitude no formalismo de segunda ordem que obtemos.

\footnotetext{
${ }^{3}$ É necessário mencionar que esses fatores de cores não são linearmente independentes porque eles satisfazem a identidade de Jacobi: $f^{m_{1} m_{2} a} f^{m_{4} m_{3} a}+f^{m_{1} m_{4} a} f^{m_{3} m_{2} a}+f^{m_{1} m_{3} a} f^{m_{2} m_{4} a}=0$. Porém, a equivalência entre os formalismos de primeira e segunda ordem é tão direta, tal que, podemos comparar termo a termo.

${ }^{4}$ Ele foi omitido na computação explícita assim como o fator de $g^{2}$ da constante de acoplamento.

${ }^{5}$ Note que são apenas os diagramas do tipo II que possuem alguma dependência com o parâmetro de calibre, pois o propagador interno é o propagador do campo de calibre $A_{\mu}^{a}$. Ressaltamos que o propagador interno também pode ser usado para classificação dos diagramas; onde diagramas do tipo 0 seriam diagramas com propagadores internos do campo auxiliar, já do tipo I teriam propagadores mistos internos e do tipo II, o propagador do campo de calibre. Embora essa seja mais fácil de ser generalizada, nesse caso a classificação por número de pernas externas mistas é mais prática.
} 


\title{
Espalhamento Glúon - Glúon
}

\author{
$\ln [0]:=<$ FeynCalc ${ }^{\prime}$;
}

FeynCalc 9.3.1 (development version). For help, use the

documentation center, check out the wiki or visit the forum.

\section{Regras de Feynman}

$\ln [\circ]:=(*$ Abreviações básicas das definições $*)$

$\ln [\circ]=\mathrm{mt}\left[\mu_{-}, v_{-}\right]=$MetricTensor $[\mu, \boldsymbol{v}$, Dimension $\rightarrow \mathrm{d}]$;

$\mathrm{fv}\left[\mathrm{p}_{-}, \mu_{-}\right]=$FourVector $[\mathrm{p}, \mu$, Dimension $\rightarrow \mathrm{d}]$;

$s p\left[p_{-}, q_{-}\right]=$ScalarProduct $[p, q$, Dimension $\rightarrow d]$;

$\ln [\circ]=L f\left[m 1_{-}, \mathrm{m} 2_{-}, \mathrm{m} 3_{-}, \mathrm{m} 4_{-}, q_{-}\right]=$

$-(f v[q, m 1] \times f v[q, m 3] \times m t[m 2, m 4]+f v[q, m 2] \times f v[q, m 4] \times m t[m 1, m 3]-f v[q, m 1] \times f v[q, m 4] \times m t[m 2, m 3]-$

$\mathrm{fv}[\mathrm{q}, \mathrm{m} 2] \times \mathrm{fv}[\mathrm{q}, \mathrm{m} 3] \times \mathrm{mt}[\mathrm{m} 1, \mathrm{~m} 4]) / 2 / \mathrm{sp}[\mathrm{q}, \mathrm{q}]$

Outfo $J=\frac{-q^{\mathrm{m} 1} q^{\mathrm{m} 3} g^{\mathrm{m} 2 \mathrm{~m} 4}+q^{\mathrm{m} 2} q^{\mathrm{m} 3} g^{\mathrm{m} 1 \mathrm{~m} 4}+q^{\mathrm{m} 1} q^{\mathrm{m} 4} g^{\mathrm{m} 2 \mathrm{~m} 3}-q^{\mathrm{m} 2} q^{\mathrm{m} 4} g^{\mathrm{m} 1 \mathrm{~m} 3}}{2 q^{2}}$

$\operatorname{In}[\circ]=\mathrm{IF}\left[\mathrm{m} 1_{-}, \mathrm{m} 2_{-}, \mathrm{m} 3_{-}, \mathrm{m} 4 \_\right]=(\mathrm{mt}[\mathrm{m} 1, \mathrm{~m} 3] \times \mathrm{mt}[\mathrm{m} 2, \mathrm{~m} 4]-\mathrm{mt}[\mathrm{m} 1, \mathrm{~m} 4] \times \mathrm{mt}[\mathrm{m} 2, \mathrm{~m} 3]) / 2$

Outf $=\frac{1}{2}\left(g^{\mathrm{m} 1 \mathrm{~m} 3} g^{\mathrm{m} 2 \mathrm{~m} 4}-g^{\mathrm{m} 1 \mathrm{~m} 4} g^{\mathrm{m} 2 \mathrm{~m} 3}\right)$

$\ln [\circ]=(*$ Propagador do campo $\mathrm{A} *)$

$\ln [\circ]:=\mathrm{AA}\left[\mathrm{m} 1_{-}, \mathrm{m} 2_{-}, \mathrm{q}_{-}\right]=-\mathrm{I}(\mathrm{mt}[\mathrm{m} 1, \mathrm{~m} 2]-\mathrm{al} \mathrm{fv}[\mathrm{q}, \mathrm{m} 1] \times \mathrm{fv}[\mathrm{q}, \mathrm{m} 2] / \mathrm{sp}[\mathrm{q}, \mathrm{q}]) / \mathrm{sp}[\mathrm{q}, \mathrm{q}]$

Outfo $=-\frac{i\left(g^{\mathrm{m} 1 \mathrm{~m} 2}-\frac{\mathrm{al} q^{\mathrm{m1}} q^{\mathrm{m} 2}}{q^{2}}\right)}{q^{2}}$

$\ln [\odot]=\mathrm{VF}\left[\mathrm{m} 1_{-}, \mathrm{m} 2_{-}, \mathrm{m} 3_{-}, \mathrm{m} 4 \_\right]=-\mathrm{I} / 2(\mathrm{mt}[\mathrm{m} 1, \mathrm{~m} 3] \times \mathrm{mt}[\mathrm{m} 2, \mathrm{~m} 4]-\mathrm{mt}[\mathrm{m} 1, \mathrm{~m} 4] \times \mathrm{mt}[\mathrm{m} 2, \mathrm{~m} 3])$

Outf $\circ=-\frac{1}{2} i\left(g^{\mathrm{m} 1 \mathrm{~m} 3} g^{\mathrm{m} 2 \mathrm{~m} 4}-g^{\mathrm{m} 1 \mathrm{~m} 4} g^{\mathrm{m} 2 \mathrm{~m} 3}\right)$

$\ln [0]:=(*$ Propagador do campo $\mathrm{F} *)$

$\ln [\circ]=F F\left[m 1_{-}, \mathrm{n} 1_{-}, \mathrm{m} 2_{-}, \mathrm{n} 2_{-}, \mathrm{q}_{-}\right]=2 \mathrm{I}(\mathrm{IF}[\mathrm{m} 1, \mathrm{n} 1, \mathrm{~m} 2, \mathrm{n} 2]+\mathrm{Lf}[\mathrm{m} 1, \mathrm{n} 1, \mathrm{~m} 2, \mathrm{n} 2, \mathrm{q}])$

Outfo $=2 \dot{i}\left(\frac{1}{2 q^{2}}\left(-q^{m 1} q^{m 2} g^{n 1 n 2}+q^{m 2} q^{n 1} g^{m 1 n 2}+q^{m 1} q^{n 2} g^{m 2 n 1}-q^{n 1} q^{n 2} g^{m 1 m 2}\right)+\frac{1}{2}\left(g^{m 1 m 2} g^{n 1 n 2}-g^{m 1 n 2} g^{m 2 n 1}\right)\right)$

$\ln [0]=(*$ Propagador misto FA e AF *)

$\ln [\odot]=\mathrm{FA}\left[\mathrm{m} 1_{-}, \mathrm{m} 2_{-}, \mathrm{n} 1_{-}, \mathrm{p}_{-}\right]=(\mathrm{fv}[\mathrm{p}, \mathrm{m} 1] * \mathrm{mt}[\mathrm{m} 2, \mathrm{n} 1]-\mathrm{fv}[\mathrm{p}, \mathrm{m} 2] * \mathrm{mt}[\mathrm{m} 1, \mathrm{n} 1]) / \mathrm{sp}[\mathrm{p}, \mathrm{p}]$

Out: $=\frac{p^{\mathrm{m} 1} g^{\mathrm{m} 2 \mathrm{n} 1}-p^{\mathrm{m} 2} g^{\mathrm{m} 1 \mathrm{n} 1}}{p^{2}}$ 
2 | AAA__final.nb

$\ln [\circ]:=A F\left[n 1_{-}, m 1_{-}, m 2_{-}, p_{-}\right]=-(f v[p, m 1] * m t[m 2, n 1]-f v[p, m 2] * m t[m 1, n 1]) / s p[p, p]$

Out $\circ]=\frac{p^{\mathrm{m} 2} g^{\mathrm{m} 1 \mathrm{n} 1}-p^{\mathrm{m} 1} g^{\mathrm{m} 2 \mathrm{n} 1}}{p^{2}}$

\section{Amplitude de 4 glúons no formalismo de segunda ordem de YM}

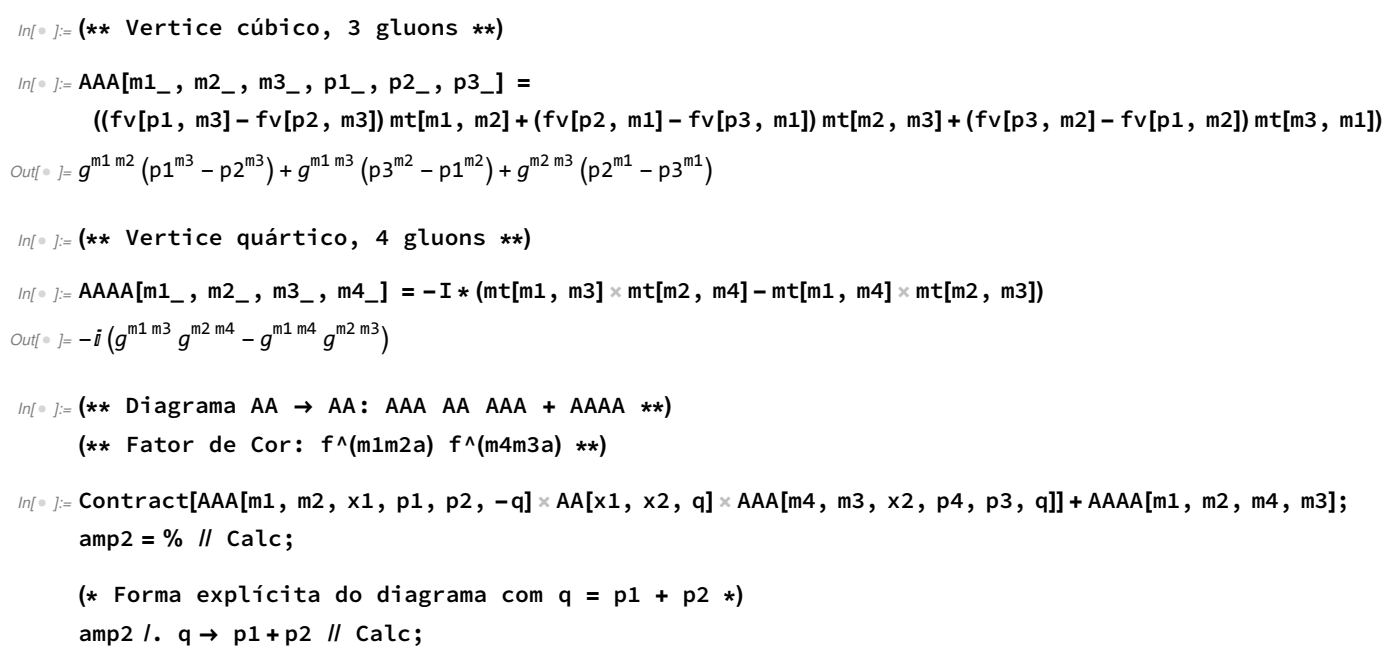

\section{Amplitude de 4 glúons no formalismo de primeira ordem de YM}

$\operatorname{In}[\circ]:=(* *$ Diagrama TO: Sem pernas mistas externas, propagador interno $\mathrm{FF} * *)$

$(* *$ AAF FF FAA**)

$\operatorname{In}[\circ]:=\operatorname{amp} 1 \mathrm{~d} \theta=\mathrm{VF}[\mathrm{m} 1, \mathrm{~m} 2, \mathrm{~m} 11, \mathrm{~m} 22] \times \mathrm{FF}[\mathrm{m} 11, \mathrm{~m} 22, \mathrm{~m} 33, \mathrm{~m} 44, \mathrm{q}] \times \mathrm{VF}[\mathrm{m} 44, \mathrm{~m} 33, \mathrm{~m} 3, \mathrm{~m} 4] / / \mathrm{Calc}$

Outt $\circ=\frac{i q^{\mathrm{m} 1} q^{\mathrm{m} 4} g^{\mathrm{m} 2 \mathrm{~m} 3}}{q^{2}}-\frac{i q^{\mathrm{m} 1} q^{\mathrm{m} 3} g^{\mathrm{m} 2 \mathrm{~m} 4}}{q^{2}}+\frac{i q^{\mathrm{m} 2} q^{\mathrm{m} 3} g^{\mathrm{m} 1 \mathrm{~m} 4}}{q^{2}}-\frac{i j q^{\mathrm{m} 2} q^{\mathrm{m} 4} g^{\mathrm{m} 1 \mathrm{~m} 3}}{q^{2}}-i g^{\mathrm{m} 1 \mathrm{~m} 4} g^{\mathrm{m} 2 \mathrm{~m} 3}+i j g^{\mathrm{m} 1 \mathrm{~m} 3} g^{\mathrm{m} 2 \mathrm{~m} 4}$

$\ln [\circ]:=$ (* Diagramas T1: Uma perna externa mista, propagador interno misto AF, FA *)

$(*$ AAF FA AA $\left.\left.(F \rightarrow A)+\ldots *)(*(F \rightarrow A) \Leftrightarrow F) \times F A \times[A A]^{\wedge}(-1) \sim F\right) \times i p^{\wedge} 2 *\right)$

$\ln [\circ]:=d p m=V F[m 1, m 2, n 1, n 2] * F A[n 1, n 2, i 1, q] * V F[i 1, m 4, n 3, n 4] * F A[n 3, n 4, m 3,-p 3] * I s p[p 3, p 3]-$

$V F[m 1, m 2, n 1, n 2] * F A[n 1, n 2, i 1, q] * V F[i 1, m 3, n 3, n 4] * F A[n 3, n 4, m 4,-p 4] * I s p[p 4, p 4]+$

$V F[m 4, m 3, n 1, n 2] * F A[n 1, n 2, i 1,-q] * V F[i 1, m 1, n 3, n 4] * F A[n 3, n 4, m 2,-p 2] * I$ sp[p2, p2]-

$V F[m 4, m 3, n 1, n 2] * F A[n 1, n 2, i 1,-q] * V F[i 1, m 2, n 3, n 4] * F A[n 3, n 4, m 1,-p 1] * I s p[p 1, p 1] / / C a l c ;$

$\ln [\circ]:=(*$ Diagramas T2: Duas pernas externas mixtas, propagador interno $A A *)$

$(*(A \rightarrow F) A A$ AA AA $(F \rightarrow A)+\ldots *)\left(*(A \rightarrow F) \Leftrightarrow[A A]^{\wedge}(-1) \times A F \times(F *)\right.$ 
$\ln [\circ]:=\operatorname{dpg} 1=-A F[m 2, n 1, n 2, p 2] * V F[n 1, n 2, m 1, i 1] * A A[i 1, i 2, q] * V F[n 3, n 4, m 4, i 2] *$

$F A[n 3, n 4, m 3,-p 3] * s p[p 2, p 2] * s p[p 3, p 3]+A F[m 1, n 1, n 2, p 1] * V F[n 1, n 2, m 2, i 1] *$

$A A[i 1, i 2, q] * V F[n 3, n 4, m 4, i 2] * F A[n 3, n 4, m 3,-p 3] * s p[p 1, p 1] * s p[p 3, p 3]-$

$A F[m 1, n 1, n 2, p 1] * V F[n 1, n 2, m 2, i 1] * A A[i 1, i 2, q] * V F[n 3, n 4, m 3, i 2]$ *

$F A[n 3, n 4, m 4,-p 4] * s p[p 1, p 1] * s p[p 4, p 4]+A F[m 2, n 1, n 2, p 2] * V F[n 1, n 2, m 1, i 1] *$

$A A[i 1, i 2, q] * V F[n 3, n 4, m 3, i 2] * F A[n 3, n 4, m 4,-p 4] * s p[p 2, p 2] * s p[p 4, p 4] / / C a l c ;$

$\ln [*]:=(*$ Equivalência Geral para $q=p 1+p 2=-(p 3+p 4) *)$

amp $1=d p m+d p g 1+\operatorname{amp} 1 d 0 / /$ Calc ;

amp2 - amp1 // Calc;

amp2 - amp1 $=0 /$ /. q $\rightarrow$ p1 + p2 // Calc

Out $0 \mathrm{~J}=$ True

\section{Função de Green de 4-pontos}

$\ln \left[0^{0}:=\right.$ (* Equivalência da função de Green de 4-pontos no nível árvore *)

$\ln [\circ]:=$ (* Função de Green no formalismo de primeira ordem *)

$\operatorname{In}[\circ]:=\mathrm{FG} 4 \mathrm{p} 10 \mathrm{rd}=\operatorname{amp} 1 * \mathrm{AA}[\mathrm{m} 1, \mu 1, \mathrm{p} 1] * \mathrm{AA}[\mathrm{m} 2, \mu 2, \mathrm{p} 2] * \mathrm{AA}[\mathrm{m} 3, \mu 3, \mathrm{p} 3] * \mathrm{AA}[\mathrm{m} 4, \mu 4, \mathrm{p} 4] / /$ Contract;

$\ln [\circ]:=(*$ Função de Green no formalismo de segunda ordem *)

$\operatorname{In}[\circ]:=\mathrm{FG} 4 \mathrm{p} 2$ ord $=\operatorname{amp} 2 * \mathrm{AA}[\mathrm{m} 1, \mu 1, \mathrm{p} 1] * \mathrm{AA}[\mathrm{m} 2, \mu 2, \mathrm{p} 2] * \mathrm{AA}[\mathrm{m} 3, \mu 3, \mathrm{p} 3] * \mathrm{AA}[\mathrm{m} 4, \mu 4, \mathrm{p} 4] / /$ Contract;

$\ln [\circ]:=$ FG4p2ord - FG4p1ord $=\mathbf{=}$

(* Não precisamos usar a conservação de momento para determinar "q",

ou seja, o momento interno "q" é considerado uma variável independente. *)

Out 0 ]= True 



\section{REFERÊNCIAS BIBLIOGRÁfICAS}

[1] M. D. Schwartz, Quantum Field Theory and the Standard Model (Cambridge University Press, Cambridge, England, 2014).

[2] C.-N. Yang and R. L. Mills, Phys. Rev. 96, 191 (1954).

[3] L. Landau and E. Lifschitz, The Classical Theory of Fields, Vol. 2 of Course of Theoretical Physics (Pergamon Press, Oxford, 1975).

[4] J. Niederle, V. Popov, and L. Hlavatỳ, Functional Integrals in Quantum Field Theory and Statistical Physics, Mathematical Physics and Applied Mathematics (Springer, Netherlands, 1983).

[5] F. T. Brandt and D. G. C. McKeon, Phys. Rev. D91, 105006 (2015).

[6] F. T. Brandt and D. G. C. McKeon, Phys. Rev. D 93, 105037 (2016).

[7] D. G. C. McKeon, Can. J. Phys. 72, 601 (1994).

[8] J. Frenkel and J. C. Taylor, Annals Phys. 387, 1 (2017).

[9] F. T. Brandt, J. Frenkel, and D. G. C. McKeon, Phys. Rev. D98, 025024 (2018).

[10] I. 1. Buchbinder and I. 1. Shapiro, Yad. Fiz. 37, 248 (1983).

[11] I. L. Buchbinder and I. L. Shapiro, Acta Phys. Polon. B16, 103 (1985).

[12] M. Yu Kalmykov, P. I. Pronin, and K. V. Stepanyantz, Class. Quant. Grav. 11, 2645 (1994).

[13] L. D. Faddeev and V. N. Popov, Sov. Phys. Usp. 16, 777 (1974).

[14] J. S. Schwinger, Proc. Nat. Acad. Sci. 37, 452 (1951).

[15] D. G. C. McKeon, F. T. Brandt, J. Frenkel, and S. Martins-Filho, Phys. Rev. D 101, 085013 (2020).

[16] F. T. Brandt, J. Frenkel, S. Martins-Filho, and D. G. C. McKeon, Phys. Rev. D 102, 045013 (2020).

[17] J. Zinn-Justin, Quantum Field Theory and Critical Phenomena, fourth ed. (Clarendon Press, Oxford, 2002).

[18] M. Ferraris, M. Francaviglia, and C. Reina, Gen. Rel. Grav. 14, 243 (1982).

[19] L. D. Faddeev and V. N. Popov, Phys. Lett. B25, 29 (1967). 
[20] L. H. Ryder, Quantum Field Theory (Cambridge University Press, Cambridge, England, 1994).

[21] M. E. Peskin and D. V. Schroeder, An introduction to quantum field theory, Frontiers in Physics (Perseus Books, Reading, Massachusetts, 1995).

[22] A. Das, Field Theory: A Path Integral Approach (World Scientific, NY, 1993).

[23] R. P. Feynman and A. R. Hibbs, Quantum Mechanics and Path Integrals (McGraw-Hill, New York, 1965).

[24] A. Pilaftsis, Physical Review D 56, 5431 (1997).

[25] M. Frank, T. Hahn, S. Heinemeyer, W. Hollik, H. Rzehak, and G. Weiglein, J. High Energy Phys. 2007, 047 (2007).

[26] J. Chisholm, Nuclear Physics 26, 469 (1961).

[27] L. F. Abbott, Acta Phys. Polon. B13, 33 (1982).

[28] K. G. Wilson and W. Zimmermann, Commun. Math. Phys. 24, 87 (1972).

[29] Y. Takahashi, Nuovo Cim. 6, 371 (1957).

[30] F. J. Dyson, Phys. Rev. 75, 1736 (1949).

[31] I. Shapiro, Physics Reports 357, 113 (2002).

[32] D. M. Capper, G. Leibbrandt, and M. Ramon Medrano, Phys. Rev. D8, 4320 (1973).

[33] D. M. Capper and M. R. Medrano, Phys. Rev. D9, 1641 (1974).

[34] J. F. Donoghue, Phys. Rev. D 50, 3874 (1994).

[35] N. Dadhich and J. M. Pons, General Relativity and Gravitation 44, 2337 (2012).

[36] A. Edery and Y. Nakayama, Phys. Rev. D 99, 124018 (2019).

[37] M. H. Lagraa, AIP Conference Proceedings 1295, 222 (2010).

[38] G. Leibbrandt, Reviews of Modern Physics 47, 849 (1975).

[39] C. G. Bollini and J. J. Giambiagi, Nuovo Cim. B12, 20 (1972).

[40] F. T. Brandt, J. Frenkel, S. Martins-Filho, and D. G. C. McKeon, Ann. Phys. (N. Y.) 168426 (2021), arXiv 2009.09553.

[41] R. Utiyama, Phys. Rev. 101, 1597 (1956).

[42] D. Ivanenko and G. Sardanashvily, Physics Reports 94, 1 (1983).

[43] F. W. Hehl, J. McCrea, E. W. Mielke, and Y. Ne'eman, Physics Reports 258, 1 (1995).

[44] L. Nachbin, Proc. Amer. Math. Soc. 12, 11 (1961).

[45] E. Witten, Comm. Math. Phys. 137, 29 (1991).

[46] H. Georgi and S. L. Glashow, Phys. Rev. Lett. 32, 438 (1974).

[47] C. Beck and M. C. Mackey, Physica A379, 101 (2007). 
[48] J. I. Kapusta, Finite Temperature Field Theory (Cambridge University Press, Cambridge, England, 1989).

[49] P. Arnold and C. Zhai, Phys. Rev. D 50, 7603 (1994).

[50] D. G. Boulware and L. S. Brown, Phys. Rev. 172, 1628 (1968).

[51] S. Weinberg, Quantum Theory of Fields II (Benjamin Cummings, Cambridge, 1995).

[52] B. R. Holstein and J. F. Donoghue, Phys. Rev. Lett. 93, 201602 (2004).

[53] G. 't Hooft and M. Veltman, Nucl. Phys. B 44, 189 (1972).

[54] G. Passarino and M. Veltman, Nucl. Phys. B 160, 151 (1979).

[55] R. Mertig, M. Böhm, and A. Denner, Computer Physics Communications 64, 345 (1991). 
